Supporting Information (https://doi.org/10.1021/acs.jmedchem.1c00679)

\title{
The Discovery of IACS-9779 and IACS-70465 as Potent Inhibitors Targeting Indoleamine 2,3-Dioxygenase 1 (IDO1) Apoenzyme
}

Matthew M. Hamilton*1, Faika Mseeh ${ }^{1}$, Timothy J. McAfoos ${ }^{1}$, Paul G. Leonard ${ }^{1}$, Naphtali J. Reyna $^{1}$, Angela L. Harris ${ }^{2}$, Alan Xu ${ }^{1}$, Michelle Han ${ }^{1}$, Michael J. Soth ${ }^{1}$, Barbara Czako ${ }^{1}$, Jay P. Theroff $^{1}$, Pijus K Mandal ${ }^{1}$, Jason P. Burke ${ }^{1}$, Brett Virgin-Downey ${ }^{1}$, Alessia Petrocchi ${ }^{1}$, Dana Pfaffinger $^{1}$, Norma E. Rogers ${ }^{1}$, Connor A. Parker ${ }^{1}$, Simon S. Yu ${ }^{1}$, Yongying Jiang ${ }^{1}$, Stephan Krapp $^{3}$, Alfred Lammens ${ }^{3}$, Graham Trevitt ${ }^{4}$, Martin R. Tremblay ${ }^{5}$, Keith Mikule ${ }^{5}$, Keith Wilcoxen $^{5}$, Jason B. Cross ${ }^{1}$, Philip Jones ${ }^{1}$, Joseph . R. Marszalek ${ }^{2}$, and Richard T. Lewis ${ }^{1}$.

*Matthew M. Hamilton - IACS (Institute for Applied Cancer Science, University of Texas, MD Anderson Cancer Center, 1881 East Road, Houston, TX, 77054 United States; Email: mmhamilton@mdanderson.org

Table of contents

1) Synthetic experimentals for compounds 18 to 20, 23 to 61, 63 to 69, and 72.

2) Western-blot of IDO1

3) Microsomal stability assay.

4) CYP Inhibition Assays.

5) Plasma Protein Binding Assay

6) hERG Fluorescence Polarization Assay.

7) hERG Qpatch (Automated Electrophysiology) Assay.

8) hERG Manual Patch Clamp Assay

9) X-ray crystallography procedure.

10) Sequence Alignment of the Binding Site of IDO1

11) HeLa Cellular Assay Curves for 22, and 62 (IACS9779).

12) LCMS of Lead Compounds

13) References

\section{Page}

$\mathrm{S} 1-\mathrm{S} 50$

$\mathrm{S} 50$

S50

S50 - S51

S51 - S52

S52

S52 - S55

S55 - S57

S57 - S58

$\mathrm{S} 58-\mathrm{S} 59$

S59 - S60

S60 - S79

S80 


\section{1) Synthetic Experimentals}

Compound 18: $\quad$ N-(4-chlorophenyl)-2-(3-((quinolin-4-yloxy)methyl)azetidin-1yl)propanamide.

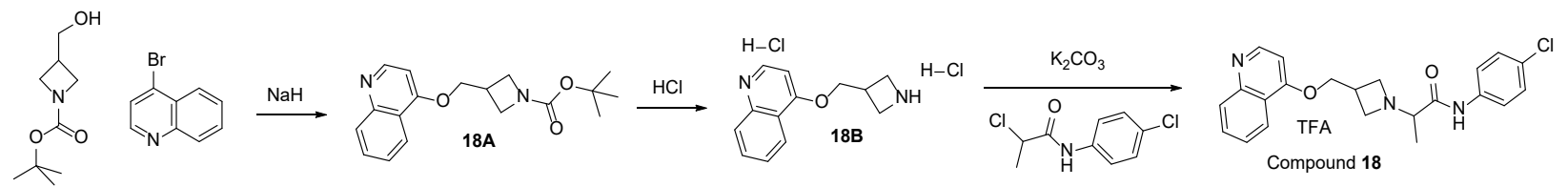

Compound 18A: tert-butyl 3-((quinolin-4-yloxy)methyl)azetidine-1-carboxylate. To a solution of tert-butyl 3-(hydroxymethyl)azetidine-1-carboxylate (200 mg, $1.07 \mathrm{mmol})$ in DMSO (5.3 mL) was added $\mathrm{NaH}$ (85 mg, $2.14 \mathrm{mmol}, 60 \%$ dispersion in mineral oil) portion wise and the resulting mixture was stirred at room temperature for $15 \mathrm{~min}$. To the mixture was added 4-bromoquinoline (267 $\mathrm{mg}, 1.28 \mathrm{mmol}$ ) and the reaction heated at $80{ }^{\circ} \mathrm{C}$ until completion. The reaction was diluted with saturated $\mathrm{NH}_{4} \mathrm{Cl}$ and extracted with EtOAc $(3 \mathrm{x})$, the organics combined, dried over $\mathrm{MgSO}_{4}$, filtered, and concentrated. The residue was adsorbed onto silica gel and purified via flash chromatography (0-50 \% EtOAc:IPA (8:2) in hexanes) to give the title compound 6A (190 mg, $0.60 \mathrm{mmol}, 56 \%$ yield) as an off white light yellow solid. MS (ES $\left.{ }^{+}\right) \mathrm{C}_{18} \mathrm{H}_{22} \mathrm{~N}_{2} \mathrm{O} 3$ requires: 314, found: $315[\mathrm{M}+\mathrm{H}]^{+} .{ }^{1} \mathrm{H}$ NMR (600 MHz, DMSO-d6) $\delta \mathrm{ppm} 8.74(\mathrm{~d}, J=5.29 \mathrm{~Hz}, 1 \mathrm{H}), 8.12(\mathrm{~d}, J$ $=8.31 \mathrm{~Hz}, 1 \mathrm{H}), 7.96(\mathrm{~d}, J=8.31 \mathrm{~Hz}, 1 \mathrm{H}), 7.78-7.72(\mathrm{~m}, 1 \mathrm{H}), 7.54(\mathrm{t}, J=7.55 \mathrm{~Hz}, 1 \mathrm{H}), 7.04(\mathrm{~d}$, $J=4.91 \mathrm{~Hz}, 1 \mathrm{H}), 4.38$ (d, $J=5.29 \mathrm{~Hz}, 2 \mathrm{H}), 4.04$ (br. s., 2H), 3.84 (br. s., 2H), 3.05 - 3.18 (m, 1H), $1.41(\mathrm{~s}, 9 \mathrm{H})$.

Compound 18B: 4-(azetidin-3-ylmethoxy)quinoline dihydrochloride. Tert-butyl 3-((quinolin-4yloxy)methyl)azetidine-1-carboxylate 7A (188 mg, $0.598 \mathrm{mmol})$ dissolved in DCM and cooled in an ice bath was added $4 \mathrm{M} \mathrm{HCl}$ in dioxane $(1.50 \mathrm{~mL}, 5.98 \mathrm{mmol})$. The reaction was stirred and allowed to warm to room temperature. Upon completion, the reaction was concentrated and residual solvent was azeotroped with mixtures of DCM/toluene and DCM/hexanes to give the title compound 6B (172 mg, $0.599 \mathrm{mmol}, 100 \%$ yield) and used directly in the next step.

Compound 18: N-(4-chlorophenyl)-2-(3-((quinolin-4-yloxy)methyl)azetidin-1-

yl)propanamide. 4-(Azetidin-3-ylmethoxy)quinoline dihydrochloride 6B (30 mg, $0.10 \mathrm{mmol})$ dissolved in DMF $(0.5 \mathrm{~mL})$ was added potassium carbonate $(72 \mathrm{mg}, 0.52)$ and 2-chloro-N-(4- 
chlorophenyl)propanamide (synthesized similar to WO2016/073738) (27 mg, $0.13 \mathrm{mmol}$ ). The reaction was sealed and heated to $60^{\circ} \mathrm{C}$ until completion. The reaction was filtered and purified by mass-triggered preparative HPLC (Mobile phase: $\mathrm{A}=0.1 \% \mathrm{TFA} / \mathrm{H} 2 \mathrm{O}, \mathrm{B}=0.1 \% \mathrm{TFA} / \mathrm{MeCN}$; Gradient: $\mathrm{B}=30-70 \% ; 12 \mathrm{~min}$; Column: $\mathrm{C} 18)$ to give the title compound as a TFA salt (4 mg, 10 $\mu$ mol, $9 \%$ yield). MS ( $\left.\mathrm{ES}^{+}\right) \mathrm{C}_{22} \mathrm{H}_{22} \mathrm{ClN}_{3} \mathrm{O}_{2}$ requires: 395, found: $396[\mathrm{M}+\mathrm{H}]^{+} .{ }^{1} \mathrm{H}$ NMR (600 MHz, $\left.\mathrm{CDCl}_{3}\right) \delta 9.01-8.93(\mathrm{~m}, 1 \mathrm{H}), 8.41(\mathrm{t}, J=7.7 \mathrm{~Hz}, 1 \mathrm{H}), 8.20(\mathrm{t}, J=7.7 \mathrm{~Hz}, 1 \mathrm{H}), 8.01$ (q, $J=7.6$ $\mathrm{Hz}, 1 \mathrm{H}), 7.80(\mathrm{q}, J=7.6 \mathrm{~Hz}, 1 \mathrm{H}), 7.54(\mathrm{t}, J=7.3 \mathrm{~Hz}, 2 \mathrm{H}), 7.45-7.37(\mathrm{~m}, 1 \mathrm{H}), 7.31(\mathrm{~d}, J=7.4$ $\mathrm{Hz}, 1 \mathrm{H}), 7.26$ (t, $J=7.9 \mathrm{~Hz}, 2 \mathrm{H}), 4.83-4.72(\mathrm{~m}, 2 \mathrm{H}), 4.61-4.54(\mathrm{~m}, 1 \mathrm{H}), 4.53-4.47(\mathrm{~m}, 1 \mathrm{H})$, $4.46-4.38$ (m, 2H), 4.33 (q, $J=8.2 \mathrm{~Hz}, 1 \mathrm{H}), 3.74-3.63$ (m, 1H), 1.55 (d, $J=6.9 \mathrm{~Hz}, 3 \mathrm{H})$.

Compound 19: N-(4-chlorophenyl)-4-(2-(quinolin-4-yl)ethyl)piperidine-1-carboxamide.
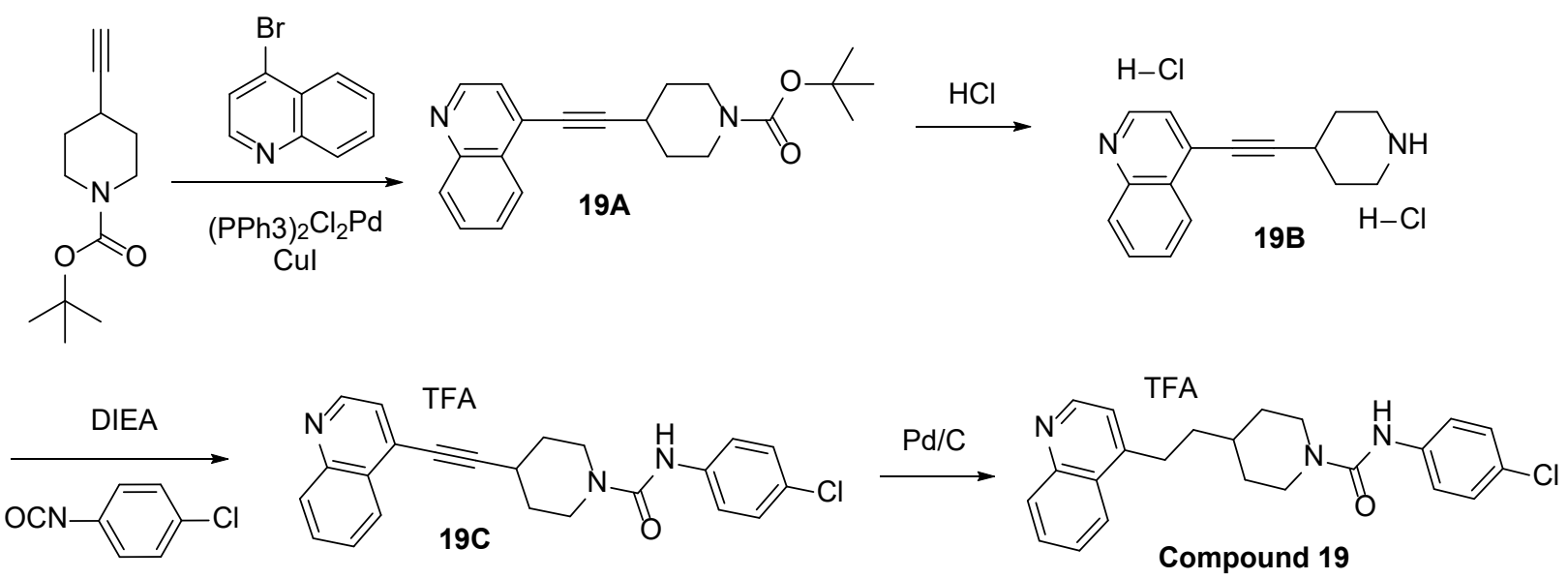

Compound 19A: tert-butyl 4-(quinolin-4-ylethynyl)piperidine-1-carboxylate. To a mixture of tert-butyl 4-ethynylpiperidine-1-carboxylate (330 mg, $1.58 \mathrm{mmol}$ ), 4-bromoquinoline (295 mg, $1.42 \mathrm{mmol}$ ), bis(triphenylphosphine)palladium(II) chloride (55.4 mg, $0.079 \mathrm{mmol})$, and CuI (15 $\mathrm{mg}, 0.079 \mathrm{mmol})$ under nitrogen was added TEA $(6 \mathrm{~mL})$ (previously purged with nitrogen for 10 min) and the reaction was heated in a dry block at $80^{\circ} \mathrm{C} 4 \mathrm{~h}$, and then $110^{\circ} \mathrm{C}$ for $2.5 \mathrm{~h}$. The reaction was concentrated, adsorbed onto silica gel and purified via flash chromatography (0-50 $\%$ EtOAc in hexanes) to give the title compound ( $457 \mathrm{mg}, 1.36 \mathrm{mmol}, 86 \%$ yield) as a light brown solid. MS (ES $\left.{ }^{+}\right) \mathrm{C}_{21} \mathrm{H}_{24} \mathrm{~N}_{2} \mathrm{O}_{2}$ requires: 336 , found: $337[\mathrm{M}+\mathrm{H}]^{+}$.

Compound 19B: 4-(piperidin-4-ylethynyl)quinoline dihydrochloride. To a solution of tert-butyl 4-(quinolin-4-ylethynyl)piperidine-1-carboxylate $(454 \mathrm{mg}, 1.35 \mathrm{mmol})$ in THF $(5 \mathrm{~mL})$ cooled in 
an ice bath was added $4 \mathrm{M} \mathrm{HCl}$ in Dioxane $(3.4 \mathrm{~mL}, 13.5 \mathrm{mmol})$ and the reaction was allowed to warm to room temperature. Upon completion the reaction was concentrated and residual solvent azeotroped with ACN and mixtures of DCM/Hexanes to give title compound and used as is in the next step. $\mathrm{C}_{16} \mathrm{H}_{16} \mathrm{~N}_{2}$ requires: 236, found: $237[\mathrm{M}+\mathrm{H}]^{+}$.

Compound 19C: N-(4-chlorophenyl)-4-(quinolin-4-ylethynyl)piperidine-1-carboxamide. To a solution of 4-(piperidin-4-ylethynyl)quinoline dihydrochloride (50 mg, $0.16 \mathrm{mmol}$ ) in DMF (1 $\mathrm{mL})$ were added DIEA $(0.11 \mathrm{~mL}, 0.65 \mathrm{mmol})$ and 1-chloro-4-isocyanatobenzene $(37 \mathrm{mg}, 0.24$ mmol) and the resulting mixture was stirred at room temperature overnight. The reaction was diluted with EtOAc, washed with water and saturated $\mathrm{NaCl}$, dried over $\mathrm{MgSO}_{4}$, filtered, concentrated, and purified by flash chromatography (0-100\% EtOAc in Hexanes) to give the title compound. $\mathrm{MS}\left(\mathrm{ES}^{+}\right) \mathrm{C}_{23} \mathrm{H}_{20} \mathrm{ClN}_{3} \mathrm{O}$ requires: 389, found: $390[\mathrm{M}+\mathrm{H}]^{+}$.

Compound 19: N-(4-chlorophenyl)-4-(2-(quinolin-4-yl)ethyl)piperidine-1-carboxamide. To a flask containing $\mathrm{N}$-(4-chlorophenyl)-4-(quinolin-4-ylethynyl)piperidine-1-carboxamide (8 mg, $0.021 \mathrm{mmol})$ dissolved in $\mathrm{MeOH}$ and purged with nitrogen was added $\mathrm{Pd} / \mathrm{C}$ (13 $\mathrm{mg}, 0.012 \mathrm{mmol}$, $10 \%$ ) and was placed under hydrogen at 1 ATM. After completion the reaction was filtered, concentrated, and purified by mass-triggered preparative HPLC (Mobile phase: $\mathrm{A}=0.1 \%$ TFA/H2O, B = 0.1\% TFA/MeCN; Gradient: $\mathrm{B}=30-70 \% ; 12 \mathrm{~min}$; Column: $\mathrm{C} 18)$ to give the title compound (6 mg, $0.012 \mathrm{mmol}, 59 \%$ yield) as a TFA white solid salt. $\mathrm{MS}\left(\mathrm{ES}^{+}\right) \mathrm{C}_{23} \mathrm{H}_{24} \mathrm{ClN}_{3} \mathrm{O}$ requires: 393, found: $394[\mathrm{M}+\mathrm{H}]^{+} .{ }^{1} \mathrm{H}$ NMR (600 MHz, DMSO) $\delta 9.00(\mathrm{~d}, J=4.9 \mathrm{~Hz}, 1 \mathrm{H}), 8.59$ $(\mathrm{s}, 1 \mathrm{H}), 8.33(\mathrm{~d}, J=8.5 \mathrm{~Hz}, 1 \mathrm{H}), 8.13(\mathrm{~d}, J=8.4 \mathrm{~Hz}, 1 \mathrm{H}), 7.98-7.92(\mathrm{~m}, 1 \mathrm{H}), 7.83-7.78(\mathrm{~m}$, $1 \mathrm{H}), 7.70(\mathrm{~d}, J=4.9 \mathrm{~Hz}, 1 \mathrm{H}), 7.50(\mathrm{~d}, J=8.8 \mathrm{~Hz}, 2 \mathrm{H}), 7.26(\mathrm{~d}, J=9.0 \mathrm{~Hz}, 2 \mathrm{H}), 4.14(\mathrm{~d}, J=13.6$ $\mathrm{Hz}, 2 \mathrm{H}), 3.26(\mathrm{dd}, J=9.5,6.4 \mathrm{~Hz}, 2 \mathrm{H}), 2.79(\mathrm{td}, J=13.0,2.6 \mathrm{~Hz}, 2 \mathrm{H}), 1.83(\mathrm{dd}, J=13.3,3.3 \mathrm{~Hz}$, 2H), $1.73-1.59(\mathrm{~m}, 3 \mathrm{H}), 1.16(\mathrm{qd}, J=12.6,4.1 \mathrm{~Hz}, 2 \mathrm{H})$.

Compound 20: N-(4-chlorophenyl)-6-(quinolin-4-yloxy)spiro[2.5]octane-1-carboxamide.

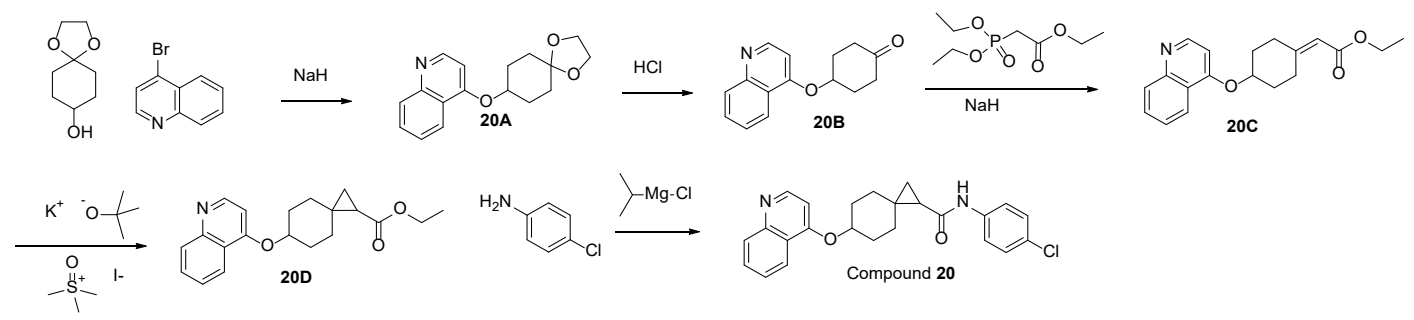


Compound 20A: 4-(1,4-dioxaspiro[4.5]decan-8-yloxy)quinoline. To a solution of 1,4dioxaspiro[4.5]decan-8-ol (1.0 g, $6.3 \mathrm{mmol})$ in DMSO $(12 \mathrm{~mL})$ cooled in ice bath was added $\mathrm{NaH}$ ( $0.51 \mathrm{~g}, 13 \mathrm{mmol}, 60 \%$ mineral oil dispersion) portion wise and the resulting mixture was stirred at room temperature for $1 \mathrm{~h}$. To the mixture was added 4-bromoquinoline (1.6 g, $7.6 \mathrm{mmol})$ and the reaction heated in a dry block at $80{ }^{\circ} \mathrm{C}$ overnight. The reaction was diluted with saturated $\mathrm{NH}_{4} \mathrm{Cl}$ and extracted with EtOAc $(3 \mathrm{x})$, the organics combined, dried over $\mathrm{MgSO}_{4}$, filtered, and concentrated. The residue was adsorbed onto silica gel and purified via flash chromatography (0$100 \%$ EtOAc in hexanes) to give the title compound $\mathbf{8 A}(1.0 \mathrm{~g}, 3.6 \mathrm{mmol}, 56 \%$ yield $)$ as a light brown solid. MS (ES $\left.{ }^{+}\right) \mathrm{C}_{17} \mathrm{H}_{19} \mathrm{NO}_{3}$ requires: 285, found: $296[\mathrm{M}+\mathrm{H}]^{+}$.

Compound 20B: 4-(quinolin-4-yloxy)cyclohexanone. To a solution of 4-(1,4dioxaspiro[4.5]decan-8-yloxy)quinoline 8A (1.0 g, $3.6 \mathrm{mmol})$ in THF $(20 \mathrm{~mL})$ was added 1M HCl (30 mL, $30.0 \mathrm{mmol}$, aqueous) and the resulting mixture was stirred at room temperature overnight. The reaction was concentrated, diluted with water and basified with $2 \mathrm{M} \mathrm{NaOH}$ and extracted with EtOAc $(3 \times 50 \mathrm{~mL})$. The organics were washed with saturated $\mathrm{NaCl}$, combined, dried over $\mathrm{MgSO}_{4}$, filtered, and concentrated. The residue was purified via flash chromatography (0-40\% 8:2 EtOAc:IPA in hexanes) to give the title compound ( $810 \mathrm{mg}, 3.4 \mathrm{mmol}, 94 \%$ yield) as a colorless solid. The NMR in DMSO showed the enol form of product and an impurity (double at $1.14 \mathrm{~min}$ ), but in $\mathrm{CD}_{3} \mathrm{OD}$ the ketone was observed with the impurity. Carry to next step as is. $\mathrm{MS}\left(\mathrm{ES}^{+}\right)$ $\mathrm{C}_{15} \mathrm{H}_{15} \mathrm{NO}_{2}$ requires: 241, found: $242[\mathrm{M}+\mathrm{H}]^{+}$.

Compound 20C: ethyl 2-(4-(quinolin-4-yloxy)cyclohexylidene)acetate. To a solution of triethyl phosphonoacetate $(0.529 \mathrm{~mL}, 2.6 \mathrm{mmol})$ in THF $(2 \mathrm{~mL})$ under $\mathrm{N}_{2}$ and cooled in an ice bath was added $\mathrm{NaH}$ (93 mg, $2.3 \mathrm{mmol}, 60 \%$ mineral oil dispersion). After addition, the reaction removed from the bath and stirred at room temperature for $30 \mathrm{~min}$. The reaction was cooled again in an ice bath and 4-(quinolin-4-yloxy)cyclohexanone 8B (375 mg, $1.5 \mathrm{mmol})$ dissolved in THF (0.5 mL) was added and the resulting mixture was stirred and allowed to warm to room temperature overnight. The reaction was concentrated, dissolved in minimal DCM, and purified by flash chromatography (0-40\% 8:2 EtOAc:IPA in hexanes) to give the title compound (520 mg, 1.2 mmol, 75\% yield) as a clear viscous liquid/oil). $M S\left(E S^{+}\right) \mathrm{C}_{19} \mathrm{H}_{21} \mathrm{NO}_{3}$ requires: 311, found: 312 $[\mathrm{M}+\mathrm{H}]^{+}$. 
Compound 20D: ethyl 6-(quinolin-4-yloxy)spiro[2.5]octane-1-carboxylate. Followed similar to literature procedure (PCT Int. Appl., 2010118207, 14 Oct 2010) from ethyl 2-(4-(quinolin-4yloxy)cyclohexylidene)acetate 6C (106 mg, $0.34 \mathrm{mmol})$, trimethylsulfoxonium iodide (150 mg, $0.68 \mathrm{mmol}$ ), and $\mathrm{KOtBu}(77 \mathrm{mg}, 0.68 \mathrm{mmol})$ in DMSO. The crude product was purified twice by flash chromatography $(10-60 \% \mathrm{EtOAc} / \mathrm{Hx})$ to give three isomers of the title compound. The third isolated material $(30 \mathrm{mg}$ ) was a mixture with starting material and the desired isomer to produce compound 8D. Each carried separately as is to next step. MS (ES $\left.{ }^{+}\right) \mathrm{C}_{20} \mathrm{H}_{23} \mathrm{NO}_{3}$ requires: 325, found: $326[\mathrm{M}+\mathrm{H}]^{+} .{ }^{1} \mathrm{H} \mathrm{NMR}\left(600 \mathrm{MHz}, \mathrm{CDCl}_{3}\right) \delta 8.72(\mathrm{~d}, J=5.2 \mathrm{~Hz}, 1 \mathrm{H}), 8.22(\mathrm{dd}, J=27.0$, $8.3 \mathrm{~Hz}, 1 \mathrm{H}), 8.02$ (dd, $J=8.3,2.3 \mathrm{~Hz}, 1 \mathrm{H}), 7.71-7.65(\mathrm{~m}, 1 \mathrm{H}), 7.49$ (dt, $J=12.0,7.6 \mathrm{~Hz}, 1 \mathrm{H})$, $6.75(\mathrm{dd}, J=9.1,5.3 \mathrm{~Hz}, 1 \mathrm{H}), 4.94-4.64(\mathrm{~m}, 1 \mathrm{H}), 4.16$ (ttd, $J=10.8,7.1,3.8 \mathrm{~Hz}, 2 \mathrm{H}), 2.66-$ $2.40(\mathrm{~m}, 1 \mathrm{H}), 2.39-2.20(\mathrm{~m}, 1 \mathrm{H}), 2.18-2.02(\mathrm{~m}, 3 \mathrm{H}), 2.02-1.70(\mathrm{~m}, 2 \mathrm{H}), 1.67-1.49(\mathrm{~m}, 2 \mathrm{H})$, $1.28(\mathrm{q}, J=8.1,7.7 \mathrm{~Hz}, 3 \mathrm{H}), 1.20(\mathrm{dd}, J=4.9 \mathrm{~Hz}, 1 \mathrm{H}), 0.95(\mathrm{dd}, J=8.0,4.4 \mathrm{~Hz}, 1 \mathrm{H})$.

Compound 20: N-(4-chlorophenyl)-6-(quinolin-4-yloxy)spiro[2.5]octane-1-carboxamide. To a cooled $0{ }^{\circ} \mathrm{C}$ solution of 4-chloroaniline ( $24 \mathrm{mg}, 0.18 \mathrm{mmol}$ ) in THF $(900 \mu \mathrm{l})$ was added isopropyl magnesium chloride ( $94 \mu 1,0.34 \mathrm{mmol}, 2 \mathrm{M}$ in THF). The resulting mixture was stirred for $5 \mathrm{~min}$, removed from the ice bath, and stirred at room temperature for $10 \mathrm{~min}$. Ethyl 6-(quinolin-4yloxy)spiro[2.5] octane-1-carboxylate $\mathbf{8 D},(30 \mathrm{mg}, 0.094 \mathrm{mmol})$ was added to the reaction mixture and stirred for $18 \mathrm{~h}$ at room temperature. The reaction mixture was diluted with EtOAc $(50 \mathrm{~mL})$, $\mathrm{H}_{2} \mathrm{O}(50 \mathrm{~mL})$ was added, and the layers were separated. The aqueous phase was extracted with EtOAc $(3 \times 50 \mathrm{~mL})$, the combined organic layers were washed with sat $\mathrm{NaCl}$, dried over $\mathrm{Na}_{2} \mathrm{SO}_{4}$, filtered and concentrated under reduced pressure. The residue was purified via silica gel chromatography $(0-100 \%$ EtOAc in hexanes) to give the title compound 8 (6.9 $\mathrm{mg}, 0.014 \mathrm{mmol}$, 14\% yield). MS ( $\left.\mathrm{ES}^{+}\right) \mathrm{C}_{24} \mathrm{H}_{23} \mathrm{NO}_{3}$ requires: 406 , found: $407[\mathrm{M}+\mathrm{H}]^{+} .{ }^{1} \mathrm{H} \mathrm{NMR}\left(600 \mathrm{MHz}, \mathrm{CDCl}_{3}\right.$ ) $\delta 8.98(\mathrm{~d}, J=6.7 \mathrm{~Hz}, 1 \mathrm{H}), 8.34(\mathrm{~d}, J=8.7 \mathrm{~Hz}, 1 \mathrm{H}), 8.31(\mathrm{~d}, J=8.5 \mathrm{~Hz}, 1 \mathrm{H}), 7.99-7.92(\mathrm{~m}, 1 \mathrm{H})$, 7.92 (s, 1H), 7.73 (t, $J=7.6 \mathrm{~Hz}, 1 \mathrm{H}), 7.49$ (d, $J=8.5 \mathrm{~Hz}, 2 \mathrm{H}), 7.23$ (d, $J=8.8 \mathrm{~Hz}, 2 \mathrm{H}), 7.08$ (d, $J$ $=6.5 \mathrm{~Hz}, 1 \mathrm{H}), 5.04-4.91(\mathrm{~m}, 1 \mathrm{H}), 2.21-2.14(\mathrm{~m}, 1 \mathrm{H}), 2.15-2.09(\mathrm{~m}, 2 \mathrm{H}), 2.09-2.02(\mathrm{~m}, 1 \mathrm{H})$, $1.95-1.81(\mathrm{~m}, 2 \mathrm{H}), 1.75-1.66(\mathrm{~m}, 1 \mathrm{H}), 1.65-1.59(\mathrm{~m}, 1 \mathrm{H}), 1.57(\mathrm{dd}, J=7.9,5.3 \mathrm{~Hz}, 1 \mathrm{H}), 1.36$ (t, $J=4.9 \mathrm{~Hz}, 1 \mathrm{H}), 1.00(\mathrm{dd}, J=8.0,4.4 \mathrm{~Hz}, 1 \mathrm{H})$. 
yl)oxy)bicyclo[3.1.0]hexan-6-yl)propyl)benzamide.
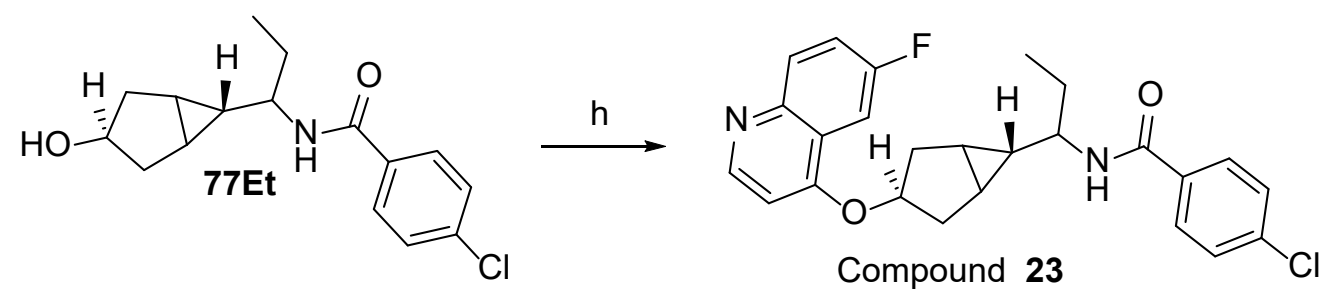

The title compound was synthesize by a similar procedure by compound 21. MS $\left(\mathrm{ES}^{+}\right)$ $\mathrm{C}_{25} \mathrm{H}_{24} \mathrm{ClFN}_{2} \mathrm{O}_{2}$ requires: 438 , found: $439[\mathrm{M}+\mathrm{H}]^{+} .{ }^{1} \mathrm{H}$ NMR (600 MHz, DMSO- $\left.d_{6}\right): \delta 9.00(\mathrm{~d}, J$ $=6.04 \mathrm{~Hz}, 1 \mathrm{H}), 8.34(\mathrm{~d}, J=8.69 \mathrm{~Hz}, 1 \mathrm{H}), 8.17(\mathrm{dd}, J=10.20,4.91 \mathrm{~Hz}, 1 \mathrm{H}), 7.94$ (dt, $J=6.14$, $2.79 \mathrm{~Hz}, 2 \mathrm{H}), 7.85(\mathrm{~d}, J=8.69 \mathrm{~Hz}, 2 \mathrm{H}), 7.53(\mathrm{~d}, J=8.31 \mathrm{~Hz}, 2 \mathrm{H}), 7.31(\mathrm{~d}, J=6.04 \mathrm{~Hz}, 1 \mathrm{H}), 5.38$ (t, $J=6.61 \mathrm{~Hz}, 1 \mathrm{H}), 3.41-3.30(\mathrm{~m}, 1 \mathrm{H}), 2.46-2.34(\mathrm{~m}, 2 \mathrm{H}), 2.15-1.99(\mathrm{~m}, 2 \mathrm{H}), 1.72-1.55(\mathrm{~m}$, 2H), $1.49-1.39(\mathrm{~m}, 2 \mathrm{H}), 1.17(\mathrm{dt}, J=8.69,3.21 \mathrm{~Hz}, 1 \mathrm{H}), 0.89(\mathrm{t}, J=7.37 \mathrm{~Hz}, 3 \mathrm{H})$.

Compound 24: 4-chloro-N-(1-((1R,3s,5S,6r)-3-((6-fluoroquinolin-4-yl)oxy)bicyclo[3.1.0]hexan6-yl)propyl)benzamide. (General Mitsunobu Procedure).<smiles>[Z7]CC1C2CC(O)CC2C1C(CC)NC(=O)c1ccc(Cl)cc1</smiles>

To a vial containing 4-chloro-N-(1-((1R,3r,5S,6r)-3-hydroxybicyclo[3.1.0]hexan-6yl)propyl)benzamide 77Et $(10 \mathrm{mg}, 0.034 \mathrm{mmol})$ dissolved in THF $(0.5 \mathrm{~mL})$ was added 6fluoroquinolin-4-ol (10.06 mg, $0.061 \mathrm{mmol}$ ) and polymer-bound $\mathrm{PPh}_{3}$, (Sigma-Aldrich 366455, 3 $\mathrm{mmol} / \mathrm{g}, 23 \mathrm{mg}, 0.068 \mathrm{mmol})$. The mixture was stirred for $10 \mathrm{~min}$ and then DTBAD (16 mg, 0.068 mmol) was added and the reaction was stirred at room temperature overnight. The reaction was diluted with DCM and the mixture filtered through a celite pad, which was then rinsed with DCM. The filtrate was concentrated, resuspended in DCM $(100 \mu \mathrm{L})$ and TFA $(200 \mu \mathrm{L})$ and stirred at room temperature for 1.5 hours. The reaction was diluted with $\mathrm{MeCN} / \mathrm{EtOH}(1: 1,2 \mathrm{~mL})$, concentrated, dissolved in $\mathrm{MeOH} / \mathrm{DMSO}$, filtered, the filter rinsed with $\mathrm{MeOH}$ and the filtrate 
purified by mass-triggered preparative HPLC (Mobile phase: $\mathrm{A}=0.1 \% \mathrm{TFA} / \mathrm{H} 2 \mathrm{O}, \mathrm{B}=0.1 \%$ TFA/MeCN; Gradient of B into A varied for 12 or $20 \mathrm{~min}$; Column: C18) to give the title compound 24 (3.9 mg, $8.9 \mu \mathrm{mol}, 17 \%$ yield) as a white solid. HRMS $\left(\mathrm{ES}^{+}\right) \mathrm{C}_{25} \mathrm{H}_{24} \mathrm{ClFN}_{2} \mathrm{O}_{2}$ requires: $439.1583[\mathrm{M}+\mathrm{H}]^{+}$, found: $439.1578[\mathrm{M}+\mathrm{H}]^{+} .{ }^{1} \mathrm{H} \mathrm{NMR}\left(600 \mathrm{MHz}, \mathrm{CDCl}_{3}\right): \delta 8.66(\mathrm{~d}, J$ $=5.29 \mathrm{~Hz}, 1 \mathrm{H}), 8.01(\mathrm{dd}, J=9.25,5.10 \mathrm{~Hz}, 1 \mathrm{H}), 7.77-7.70(\mathrm{~m}, 2 \mathrm{H}), 7.47-7.41(\mathrm{~m}, 2 \mathrm{H}), 6.67(\mathrm{~d}$, $J=5.29 \mathrm{~Hz}, 1 \mathrm{H}$ ), 5.99 (d, $J=8.69 \mathrm{~Hz}, 1 \mathrm{H}$ ), 4.66 (quin, $J=7.08 \mathrm{~Hz}, 1 \mathrm{H}$ ), $3.53-3.43$ (m, 1H), 2.52 (dd, $J=13.41,6.99 \mathrm{~Hz}, 1 \mathrm{H}), 2.46(\mathrm{dd}, J=13.60,7.18 \mathrm{~Hz}, 1 \mathrm{H}), 2.18-2.05(\mathrm{~m}, 2 \mathrm{H}), 1.79-1.71$ (m, 1H), $1.67(\mathrm{dt}, J=14.16,7.27 \mathrm{~Hz}, 1 \mathrm{H}), 1.64-1.60(\mathrm{~m}, 1 \mathrm{H}), 1.53-1.48(\mathrm{~m}, 1 \mathrm{H}), 1.02(\mathrm{t}, J=$ $7.37 \mathrm{~Hz}, 2 \mathrm{H}), 0.65(\mathrm{dt}, J=8.97,3.26 \mathrm{~Hz}, 1 \mathrm{H})$.

Compound 25: 4-chloro-N-(((1R,3r,5S,6r)-3-((6-fluoroquinolin-4-yl)oxy)bicyclo[3.1.0]hexan-6yl)methyl)benzamide
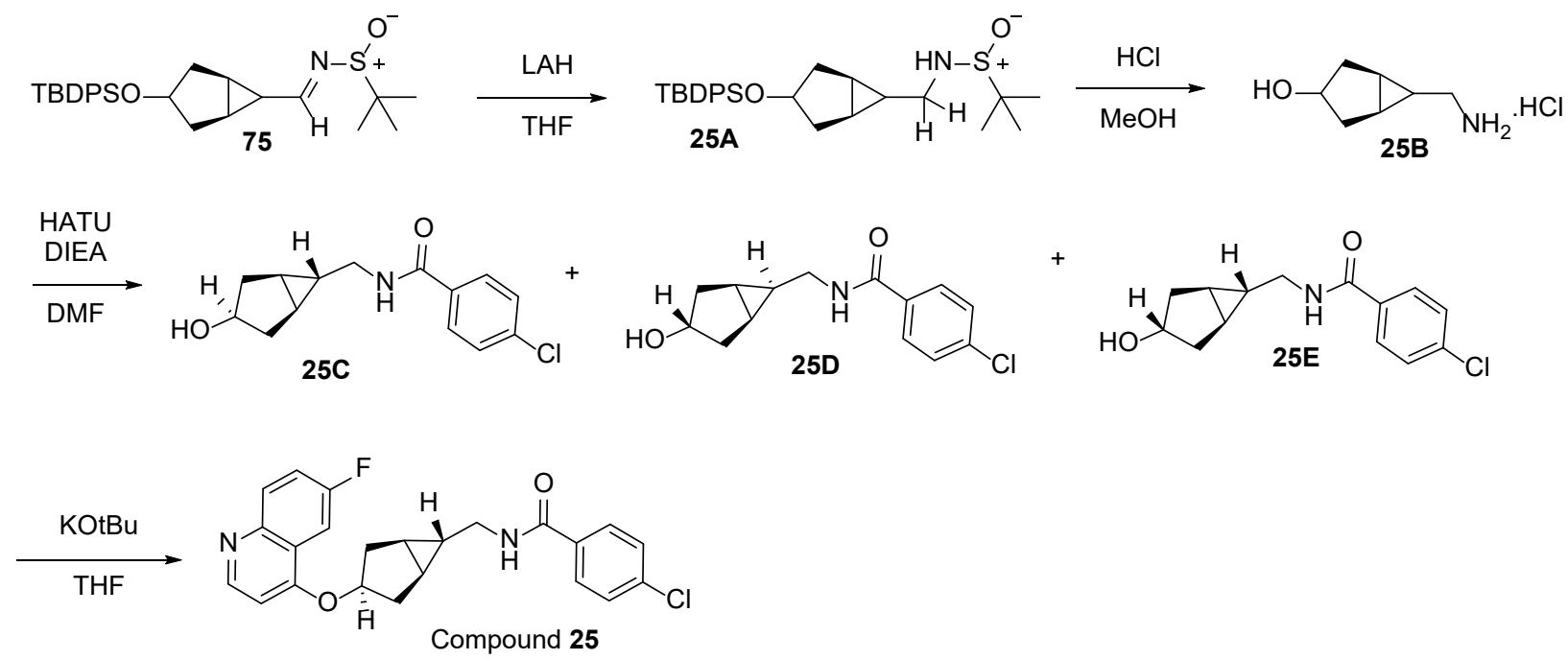

Compound 25A: N-(((1R,5S)-3-((tert-butyldiphenylsilyl)oxy)bicyclo[3.1.0] hexan-6-yl)methyl)-2methylpropane-2-sulfinamide. To a solution of (E)-N-(((1R,5S)-3-((tertbutyldiphenylsilyl)oxy)bicyclo[3.1.0]hexan-6-yl)methylene)-2-methylpropane-2-sulfinamide $\mathbf{7 5}$ (93 mg, $0.20 \mathrm{mmol})$ in THF $(1 \mathrm{~mL})$ cooled in an acetone dry ice bath was added LAH $(0.12 \mathrm{~mL}$, $0.239 \mathrm{mmol}$ ) and the resulting mixture was allowed to warm to room temperature overnight. The reaction was cooled in ice and quenched with $\mathrm{MeOH}$. The reaction was then diluted with saturated $\mathrm{NH}_{4} \mathrm{Cl}$ and extracted with EtOAc $(8 \mathrm{~mL} \times 6)$. The organic layers were washed with brine, dried 
over $\mathrm{MgSO}_{4}$, filtered and concentrated to give the title compound 25A (91 mg, $0.19 \mathrm{mmol}, 97 \%$ yield) as a clear semisolid. MS (ES ${ }^{+} \mathrm{C}_{27} \mathrm{H}_{39} \mathrm{NO}_{2} \mathrm{SSi}$ requires: 469 , found: $470[\mathrm{M}+\mathrm{H}]^{+}$.

Compound 25B: (1R,5S)-6-(aminomethyl)bicyclo[3.1.0]hexan-3-ol. $\quad \mathrm{N}-(((1 \mathrm{R}, 5 \mathrm{~S})-3-(($ tertbutyldiphenylsilyl)oxy)bicyclo[3.1.0]hexan-6-yl)methyl)-2-methylpropane-2-sulfinamide 25A was dissolved in $\mathrm{MeOH}$, cooled in an acetone dry ice bath and $\mathrm{HCl}(0.5 \mathrm{~mL}$ of $4 \mathrm{~N}$ solution in Dioxane, $2.0 \mathrm{mmol}$ ) was added and the reaction was removed from the bath (white precipitate formed) and reaction was allowed to warm to room temperature ( $\sim 5 \mathrm{~min}$ clear solution) and stirred at room temperature for $6 \mathrm{hr}$. Reaction was concentrated, and excess acidic methanol was azeotroped with addition of mixtures of $\mathrm{DCM} /$ toluene and $\mathrm{DCM} / \mathrm{Hexanes}$ to give the title compound 25B ( $25 \mathrm{mg}, 0.19 \mathrm{mmol}, 99 \%$ yield) as a white solid, used directly as is in the next step. MS (ES ${ }^{+} \mathrm{C}_{7} \mathrm{H}_{13} \mathrm{NO}$ requires: 127, found: $128[\mathrm{M}+\mathrm{H}]^{+}$.

Compounds $\quad 25 \mathrm{C}$ : $\quad$ 4-chloro- $N-(((1 R, 3 r, 5 S, 6 r)-3-h y d r o x y b i c y c l o[3.1 .0]$ hexan-6yl)methyl)benzamide. To a solution of (1R,5S)-6-(aminomethyl)bicyclo[3.1.0]hexan-3-ol hydrochloride 25B (25 mg, $0.19 \mathrm{mmol})$ in DMF (2.00 mL) were added DIEA (0.17 mL, 0.99 mmol), 4-chlorobenzoic acid (34 mg, $0.22 \mathrm{mmol}$ ) and HATU (91 mg, $0.24 \mathrm{mmol}$ ) and the resulting mixture was stirred at room temperature $18 \mathrm{~h}$. The mixture was concentrated and the residue was purified via flash chromatography on silica gel, eluent (0-90\% EtOAc in hexanes) to give the title compound $25 \mathrm{C}$ as the first eluting product ( $32 \mathrm{mg}, 0.12 \mathrm{mmol}, 61 \%$ yield) as a white solid. MS $\left(\mathrm{ES}^{+}\right) \mathrm{C}_{14} \mathrm{H}_{16} \mathrm{ClNO}_{2}$ requires: 265, found: $266[\mathrm{M}+\mathrm{H}]^{+}$.

Compound 25: 4-chloro-N-(((1R,3r,5S,6r)-3-((6-fluoroquinolin-4-yl)oxy)bicyclo[3.1.0]hexan6-yl)methyl)benzamide. The title compounds was synthesized by a similar procedure as compound 22, using compound 4-chloro-N-(((1R,3r,5S,6r)-3-hydroxybicyclo[3.1.0]hexan-6yl)methyl)benzamide $25 \mathrm{C}$ (6.6 mg, $0.025 \mathrm{mmol}$ ), 4-chloro-6-fluoroquinoline (5.41 mg, 0.030 $\mathrm{mmol}$ ), and $\mathrm{KOtBu}(5.6 \mathrm{mg}, 0.050 \mathrm{mmol})$ in THF to give the title compound $25(4.8 \mathrm{mg}, 0.012$ mmol, $47 \%$ yield) as a white solid. $\mathrm{MS}\left(\mathrm{ES}^{+}\right) \mathrm{C}_{23} \mathrm{H}_{20} \mathrm{ClFN}_{2} \mathrm{O}_{2}$ requires: 410 , found: $411[\mathrm{M}+\mathrm{H}]^{+}$. ${ }^{1} \mathrm{H}$ NMR $\left(500 \mathrm{MHz}, \mathrm{MeOD}-d_{4}\right): \delta 8.92(\mathrm{~d}, J=6.41 \mathrm{~Hz}, 1 \mathrm{H}), 8.14(\mathrm{dd}, J=9.16,4.58 \mathrm{~Hz}, 1 \mathrm{H}) 8.04$ (dd, $J=8.70,2.59 \mathrm{~Hz}, 1 \mathrm{H}), 7.93(\mathrm{td}, J=8.62,2.59 \mathrm{~Hz}, 1 \mathrm{H}), 7.79$ (d, $J=8.55 \mathrm{~Hz}, 2 \mathrm{H}), 7.45$ (d, $J$ $=8.55 \mathrm{~Hz}, 2 \mathrm{H}), 7.37(\mathrm{~d}, \mathrm{~J}=6.71 \mathrm{~Hz}, 1 \mathrm{H}), 5.45(\mathrm{t}, J=6.56 \mathrm{~Hz}, 1 \mathrm{H}), 3.26(\mathrm{~d}, J=7.02 \mathrm{~Hz}, 2 \mathrm{H})$, $2.63-2.45(\mathrm{~m}, 2 \mathrm{H}), 2.28$ (d, $J=15.26 \mathrm{~Hz}, 2 \mathrm{H}), 1.54$ (br. s., $2 \mathrm{H}), 1.39$ (td, $J=6.71,3.36 \mathrm{~Hz}, 1 \mathrm{H})$. 
Compound 26: 4-chloro-N-(((1R,3s,5S,6s)-3-((6-fluoroquinolin-4-yl)oxy)bicyclo[3.1.0]hexan6-yl)methyl)benzamide.
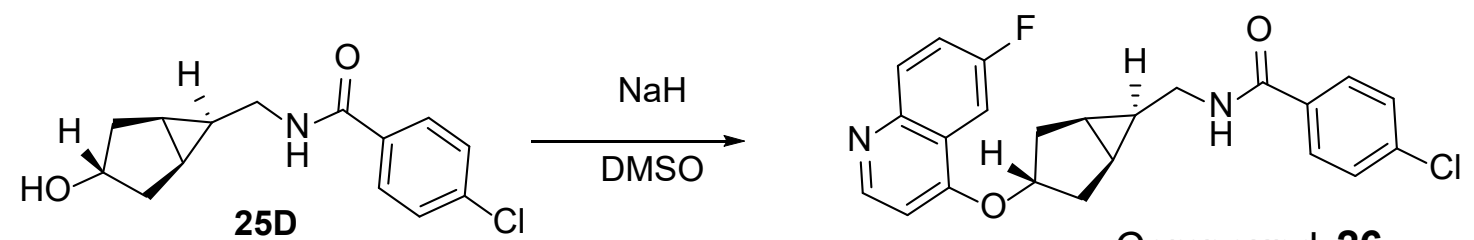

Compound 26

Compoud 25D: 4-chloro-N-(((1R,3s,5S,6s)-3-hydroxybicyclo[3.1.0]hexan-6yl)methyl)benzamide. From the synthesis of compound 25, the title compound was also isolated, (25 mg, $0.095 \mathrm{mmol}, 67 \%$ yield) as a white solid. $\mathrm{MS}\left(\mathrm{ES}^{+}\right) \mathrm{C}_{14} \mathrm{H}_{16} \mathrm{ClNO}_{2}$ requires: 265, found: $266[\mathrm{M}+\mathrm{H}]^{+}$.

Compound 26: 4-chloro-N-(((1R,3s,5S,6s)-3-((6-fluoroquinolin-4-yl)oxy)bicyclo[3.1.0]hexan6-yl)methyl)benzamide. The title compounds was synthesized by a similar procedure as compound 22 (3.7 mg, 0.009 mmol, 19\% yield) as a white solid. MS (ES $\left.{ }^{+}\right) \mathrm{C}_{23} \mathrm{H}_{20} \mathrm{ClFN}_{2} \mathrm{O}_{2}$ requires: 410, found: $411[\mathrm{M}+\mathrm{H}]^{+} .{ }^{1} \mathrm{H} \mathrm{NMR}\left(600 \mathrm{MHz}, \mathrm{CD}_{3} \mathrm{OD}\right): \delta 8.99(\mathrm{~d}, \mathrm{~J}=6.80 \mathrm{~Hz}, 1 \mathrm{H}), 8.16$ $(\mathrm{dd}, \mathrm{J}=9.44,4.53 \mathrm{~Hz}, 1 \mathrm{H}), 8.13(\mathrm{dd}, \mathrm{J}=8.88,2.83 \mathrm{~Hz}, 1 \mathrm{H}), 7.97-7.93(\mathrm{~m}, 1 \mathrm{H}), 7.86(\mathrm{~d}, \mathrm{~J}=8.31$ $\mathrm{Hz}, 2 \mathrm{H}), 7.53(\mathrm{~d}, \mathrm{~J}=6.42 \mathrm{~Hz}, 1 \mathrm{H}), 7.52$ - $7.48(\mathrm{~m}, 2 \mathrm{H}), 5.43(\mathrm{tt}, \mathrm{J}=7.37,3.97 \mathrm{~Hz}, 1 \mathrm{H}), 3.42(\mathrm{~d}, \mathrm{~J}$ $=7.93 \mathrm{~Hz}, 2 \mathrm{H}), 2.57(\mathrm{dd}, \mathrm{J}=15.30,6.99 \mathrm{~Hz}, 2 \mathrm{H}), 2.37(\mathrm{dt}, \mathrm{J}=15.86,4.53 \mathrm{~Hz}, 2 \mathrm{H}), 1.82(\mathrm{dd}, \mathrm{J}=$ 7.93, $5.67 \mathrm{~Hz}, 2 \mathrm{H}$ ), 1.29 (quin, $\mathrm{J}=8.03 \mathrm{~Hz}, 1 \mathrm{H}$ ).

Compound 27: 4-chloro-N-(((1R,3s,5S,6r)-3-((6-fluoroquinolin-4-yl)oxy)bicyclo[3.1.0]hexan6-yl)methyl)benzamide<smiles>O=C(NCC1C2C[C@@H](O)C[C@H]12)c1ccc(Cl)cc1</smiles><smiles>O=C(NC[C@H]1C2C[C@H](Oc3ccnc4ccc(F)cc34)CC21)c1ccc(Cl)cc1</smiles>

Compound 27

Compoud 25E: 4-chloro-N-(((1R,3s,5S,6r)-3-hydroxybicyclo[3.1.0]hexan-6$y$ l)methyl)benzamide. From the synthesis of compound 25, the title compound was also isolated, (6.6 mg, $0.025 \mathrm{mmol}, 12 \%$ yield) as a white solid. $\mathrm{MS}\left(\mathrm{ES}^{+}\right) \mathrm{C}_{14} \mathrm{H}_{16} \mathrm{ClNO}_{2}$ requires: 265, found: $266[\mathrm{M}+\mathrm{H}]^{+}$. 
Compound 27: 4-chloro-N-(((1R,3s,5S,6r)-3-((6-fluoroquinolin-4-yl)oxy)bicyclo[3.1.0]hexan6-yl)methyl)benzamide. The title compounds was synthesized by a similar procedure as compound 25. $\mathrm{MS}\left(\mathrm{ES}^{+}\right) \mathrm{C}_{23} \mathrm{H}_{20} \mathrm{ClFN}_{2} \mathrm{O}_{2}$ requires: 410, found: $411[\mathrm{M}+\mathrm{H}]^{+}$. ${ }^{1} \mathrm{H} \mathrm{NMR}(500 \mathrm{MHz}$, $\left.\mathrm{CD}_{3} \mathrm{OD}\right): \delta 8.93(\mathrm{~d}, J=6.41 \mathrm{~Hz}, 1 \mathrm{H}), 8.14(\mathrm{dd}, J=9.16,4.58 \mathrm{~Hz}, 1 \mathrm{H}), 8.07$ (dd, $J=8.85,2.75 \mathrm{~Hz}$, 1H), $7.96-7.89(\mathrm{~m}, 1 \mathrm{H}), 7.82(\mathrm{~d}, J=8.55 \mathrm{~Hz}, 2 \mathrm{H}), 7.52-7.45(\mathrm{~m}, 3 \mathrm{H}), 5.12(\mathrm{q}, J=6.94 \mathrm{~Hz}, 1 \mathrm{H})$, $3.24(\mathrm{~d}, J=7.02 \mathrm{~Hz}, 2 \mathrm{H}), 2.69-2.56(\mathrm{~m}, 2 \mathrm{H}), 2.29-2.16(\mathrm{~m}, 2 \mathrm{H}), 1.55$ (br. s., $2 \mathrm{H}), 1.04(\mathrm{tt}, J=$ $6.75,3.17 \mathrm{~Hz}, 1 \mathrm{H})$.

Compound 28: 4-chloro-N-((S)-1-((1R,3R,5S,6r)-3-((7-fluoroquinolin-4yl)oxy)bicyclo[3.1.0]hexan-6-yl)propyl)benzamide

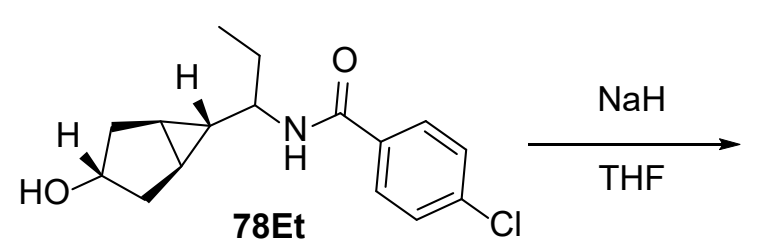<smiles>CCC(NC(=O)c1ccc(Cl)cc1)C1C2C[C@@H](Oc3ccnc4cc(F)ccc34)C[C@H]21</smiles>

Compound 28

The title compounds was synthesized by a similar procedure as compound 22. $\mathrm{MS}\left(\mathrm{ES}^{+}\right.$) $\mathrm{C}_{25} \mathrm{H}_{24} \mathrm{ClFN}_{2} \mathrm{O}_{2}$ requires: 438, found: $439[\mathrm{M}+\mathrm{H}]^{+}$. ${ }^{1} \mathrm{H} \mathrm{NMR}\left(600 \mathrm{MHz}, \mathrm{CD}_{3} \mathrm{OD}\right): \delta 8.96(\mathrm{~d}, J=$ $6.80 \mathrm{~Hz}, 1 \mathrm{H}), 8.52(\mathrm{dd}, J=9.25,5.48 \mathrm{~Hz}, 1 \mathrm{H}), 7.83-7.80(\mathrm{~m}, 2 \mathrm{H}), 7.76(\mathrm{~d}, J=8.69 \mathrm{~Hz}, 1 \mathrm{H}), 7.72$ - $7.66(\mathrm{~m}, 1 \mathrm{H}), 7.51-7.47(\mathrm{~m}, 2 \mathrm{H}), 7.44(\mathrm{~d}, J=6.80 \mathrm{~Hz}, 1 \mathrm{H}), 5.16(\mathrm{q}, J=6.89 \mathrm{~Hz}, 1 \mathrm{H}), 3.40$ (td, $\mathrm{J}=8.69,5.67 \mathrm{~Hz}, 1 \mathrm{H}), 2.62(\mathrm{dd}, J=13.41,6.99 \mathrm{~Hz}, 1 \mathrm{H}), 2.27-2.15(\mathrm{~m}, 2 \mathrm{H}), 1.84-1.75(\mathrm{~m}, 1 \mathrm{H})$, $1.74-1.67(\mathrm{~m}, 1 \mathrm{H}), 1.66-1.60(\mathrm{~m}, 1 \mathrm{H}), 1.56(\mathrm{td}, J=6.14,3.21 \mathrm{~Hz}, 1 \mathrm{H}), 1.00(\mathrm{t}, J=7.37 \mathrm{~Hz}, 3 \mathrm{H})$, $0.89(\mathrm{dt}, J=8.69,3.21 \mathrm{~Hz}, 1 \mathrm{H})$.

Compound 29: N-(1-((1R,3s,5S,6r)-3-((1,5-naphthyridin-4-yl)oxy)bicyclo[3.1.0]hexan-6yl)propyl)-4-chlorobenzamide.<smiles>CCOCCNC(CC)C1[C@H]2CC(O)C[C@H]12</smiles><smiles>CCC(NC(=O)c1ccc(Cl)cc1)[C@@H]1[C@@H]2C[C@@H]1C[C@@H]2Oc1ccnc2cccnc12</smiles>

Compound 29 
The title compound was synthesized by a similar procedure as compound 22. MS $\left(\mathrm{ES}^{+}\right)$ $\mathrm{C}_{24} \mathrm{H}_{24} \mathrm{ClN}_{3} \mathrm{O}_{2}$ requires: 421 , found: $422[\mathrm{M}+\mathrm{H}]^{+} .{ }^{1} \mathrm{H}$ NMR (DMSO- $\left.d_{6}\right): \delta 9.10-8.82(\mathrm{~m}, 2 \mathrm{H}$ ), $8.40(\mathrm{~d}, J=8.5 \mathrm{~Hz}, 1 \mathrm{H}), 8.31$ (d, $J=8.5 \mathrm{~Hz}, 1 \mathrm{H}), 7.89$ (d, $J=8.5 \mathrm{~Hz}, 3 \mathrm{H}), 7.55$ (d , $J=8.5 \mathrm{~Hz}$, 2H), 7.46 (br. s., 1H), 4.97 (d, $J=6.7 \mathrm{~Hz}, 1 \mathrm{H}), 2.59$ - 2.55 (m, 2H), $2.46-2.42$ (m, 1H), 2.07 $1.92(\mathrm{~m}, 2 \mathrm{H}), 1.64(\mathrm{dq}, J=13.4,6.6 \mathrm{~Hz}, 2 \mathrm{H}), 1.52-1.44(\mathrm{~m}, 1 \mathrm{H}), 1.44-1.35(\mathrm{~m}, 1 \mathrm{H}), 0.92(\mathrm{t}, J$ $=7.3 \mathrm{~Hz}, 3 \mathrm{H}), 0.87(\mathrm{dd}, J=8.1,3.2 \mathrm{~Hz}, 1 \mathrm{H})$.

Compound 30: N-(1-((1R,3s,5S,6r)-3-((1,6-naphthyridin-4-yl)oxy)bicyclo[3.1.0]hexan-6yl)propyl)-4-chlorobenzamide.
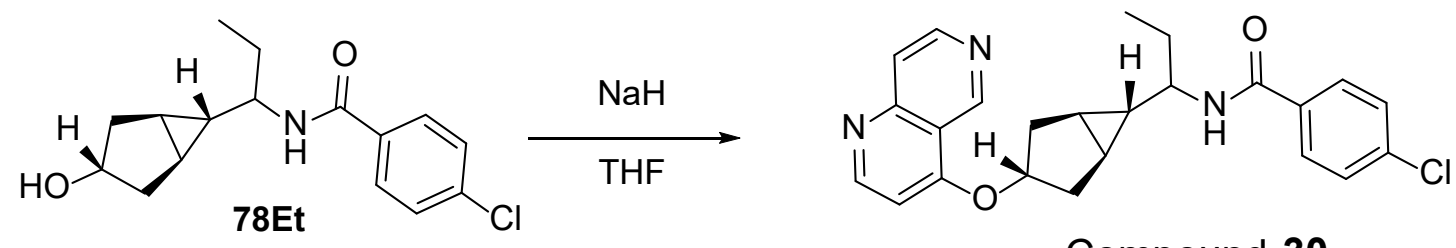

Compound 30

The title compounds was synthesized by a similar procedure as compound 22. MS (ES $)$ $\mathrm{C}_{24} \mathrm{H}_{24} \mathrm{ClN}_{3} \mathrm{O}_{2}$ requires: 421, found: $422[\mathrm{M}+\mathrm{H}]^{+} .{ }^{1} \mathrm{H} \mathrm{NMR}\left(600 \mathrm{MHz}, \mathrm{CD}_{3} \mathrm{OD}\right): \delta 9.42(\mathrm{~s}, 1 \mathrm{H})$, $8.66(\mathrm{~d}, J=6.42 \mathrm{~Hz}, 1 \mathrm{H}), 8.10(\mathrm{~d}, J=7.93 \mathrm{~Hz}, 1 \mathrm{H}), 7.86(\mathrm{~d}, J=6.80 \mathrm{~Hz}, 1 \mathrm{H}), 7.80-7.76$ (m, 1H), 7.49 - $7.43(\mathrm{~m}, 2 \mathrm{H}), 6.50(\mathrm{~d}, J=7.93 \mathrm{~Hz}, 1 \mathrm{H}$ ), 5.12 (quin, $J=6.89 \mathrm{~Hz}, 1 \mathrm{H}$ ), 3.92 (quin, $J=$ $7.55 \mathrm{~Hz}, 1 \mathrm{H}), 3.23(\mathrm{td}, J=8.69,6.04 \mathrm{~Hz}, 1 \mathrm{H}), 2.18-2.03(\mathrm{~m}, 1 \mathrm{H}), 1.74-1.57(\mathrm{~m}, 3 \mathrm{H}), 1.35-1.22$ (m, 1H), $0.94(\mathrm{t}, J=7.37 \mathrm{~Hz}, 2 \mathrm{H}), 0.71(\mathrm{dt}, J=9.25,3.12 \mathrm{~Hz}, 1 \mathrm{H})$.

Compound $\quad 31$ : 4-cyano-N-(1-((1R,3s,5S,6r)-3-((6-fluoroquinazolin-4yl)oxy)bicyclo[3.1.0]hexan-6-yl)ethyl)benzamide.
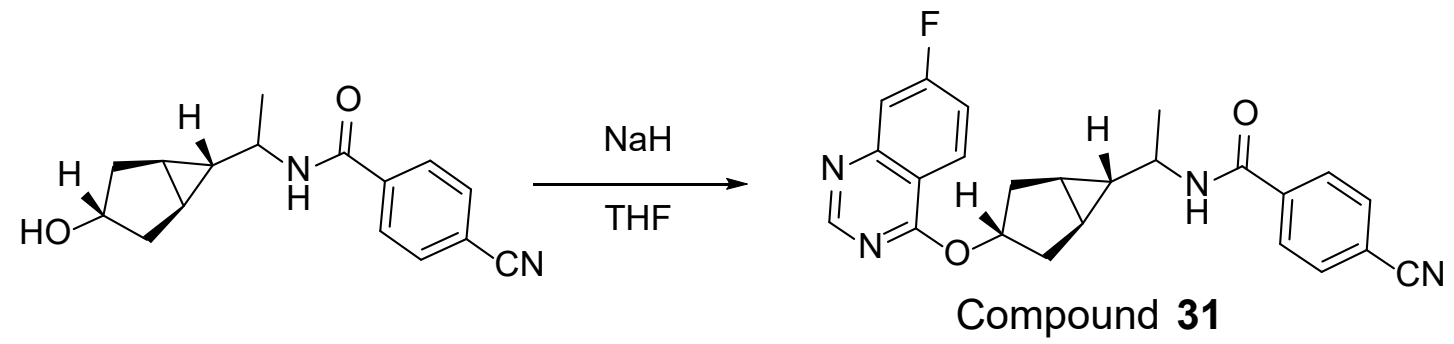

The title compounds was synthesized by a similar procedure as compound 22. HRMS (ES ${ }^{+}$) $\mathrm{C}_{24} \mathrm{H}_{21} \mathrm{FN}_{4} \mathrm{O}_{2}$ requires: $417.1721[\mathrm{M}+\mathrm{H}]^{+}$, found: $417.1713[\mathrm{M}+\mathrm{H}]^{+} .{ }^{1} \mathrm{H}$ NMR (DMSO- $d_{6}$ ): $\delta 8.78$ 
(s, 1H), $8.63(\mathrm{~d}, J=8.5 \mathrm{~Hz}, 1 \mathrm{H}), 8.24-8.21(\mathrm{~m}, 1 \mathrm{H}), 8.19-7.95(\mathrm{~m}, 4 \mathrm{H}), 7.71-7.67(\mathrm{~m}, 1 \mathrm{H})$, $7.60-7.54(\mathrm{~m}, 1 \mathrm{H}), 5.36-5.26(\mathrm{~m}, 1 \mathrm{H}), 3.50-3.42(\mathrm{~m}, 1 \mathrm{H}), 3.32(\mathrm{~s}, 1 \mathrm{H}), 2.49-2.43(\mathrm{~m}, 1 \mathrm{H})$, $2.05-1.87(\mathrm{~m}, 2 \mathrm{H}), 1.45-1.37(\mathrm{~m}, 2 \mathrm{H}), 1.24(\mathrm{~d}, J=8.1 \mathrm{~Hz}, 3 \mathrm{H}), 0.91-0.86(\mathrm{~m}, 1 \mathrm{H})$.

Compound 32: N-((1R,3s,5S,6r)-6-(1-(4-chlorobenzamido)propyl)bicyclo[3.1.0]hexan-3-yl)5-fluoronicotinamide.
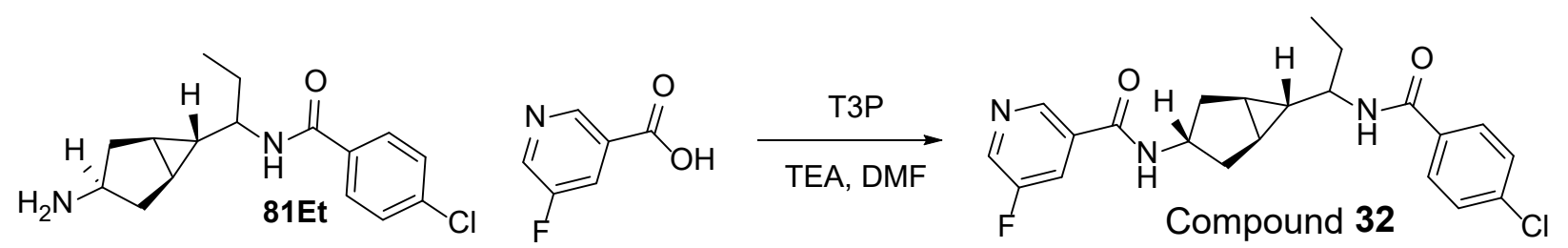

To a solution of N-(1-((1R,3r,5S,6r)-3-aminobicyclo[3.1.0]hexan-6-yl)propyl)-4chlorobenzamide 81Et $(20 \mathrm{mg}, 0.068 \mathrm{mmol})$ in DMF $(683 \mu \mathrm{l})$ were added TEA $(29 \mu 1,0.21$ mmol), 5-fluoronicotinic acid (11 mg, $0.075 \mathrm{mmol})$, and T3P (2,4,6-tripropyl-1,3,5,2,4,6trioxatriphosphinane 2,4,6-trioxide, 50\% in EtOAc, $60 \mu 1,0.10 \mathrm{mmol}$ ) and the solution stirred for 2 hours at $65^{\circ} \mathrm{C}$. The reaction was quenched with water, extracted with ethyl acetate and the organics were collected and concentrated under pressure. The residue was run on the mass directive HPLC (50-90\% $12 \mathrm{~min})$ to give the title compound 32 (9.6 mg, $0.023 \mathrm{mmol}, 33.8 \%$ yield). $\mathrm{MS}\left(\mathrm{ES}^{+}\right) \mathrm{C}_{22} \mathrm{H}_{23} \mathrm{ClFN}_{3} \mathrm{O}_{2}$ requires: 415, found: $416[\mathrm{M}+\mathrm{H}]^{+}$.

Compound 33: 4-chloro-N-(1-((1R,3s,5S,6r)-3-(5-fluoro-4-oxoquinazolin-3(4H)yl)bicyclo[3.1.0]hexan-6-yl)propyl)benzamide.
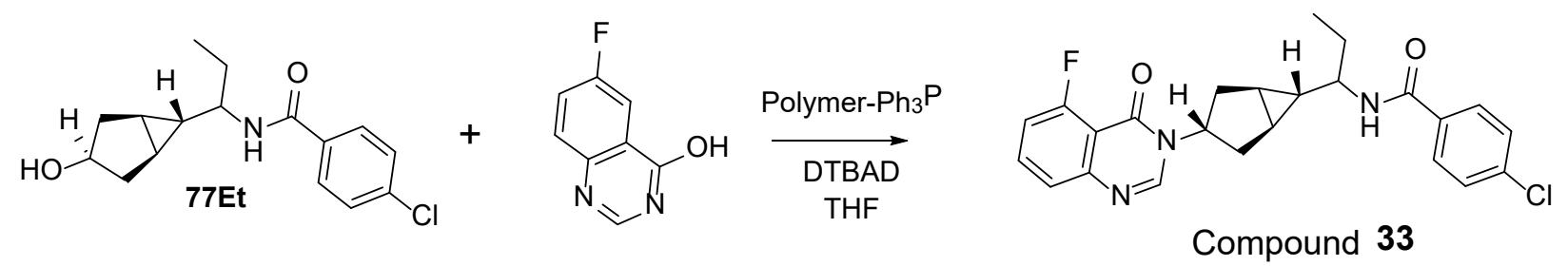

Synthesized similar to the general Mitsunobu procedure (compound 24) from 4-chloro-N-(1((1R,3r,5S,6r)-3-hydroxybicyclo[3.1.0]hexan-6-yl)propyl)benzamide 77Et (148 mg, 0.503 mmol), 5-fluoroquinazolin-4-ol (91 mg, $0.55 \mathrm{mmol})$, polymer-bound $\mathrm{PPh}_{3},(3 \mathrm{mmol} / \mathrm{g}, 335 \mathrm{mg}$, 
$1.01 \mathrm{mmol})$ and DTBAD (232 $\mathrm{mg}, 1.01 \mathrm{mmol})$ as a second eluting product from flash chromatography (0-100\% EtOAc in Hexanes) to give the title compound 33 (68 mg, $0.16 \mathrm{mmol}$, $30 \%$ yield) as a white solid. $\mathrm{MS}\left(\mathrm{ES}^{+}\right) \mathrm{C}_{24} \mathrm{H}_{23} \mathrm{ClFN}_{3} \mathrm{O}_{2}$ requires: 439 , found: $440[\mathrm{M}+\mathrm{H}]^{+} .{ }^{1} \mathrm{H}$ $\operatorname{NMR}\left(500 \mathrm{MHz}, \mathrm{CD}_{3} \mathrm{OD}\right) \delta 8.23(\mathrm{~s}, 1 \mathrm{H}), 7.80-7.76(\mathrm{~m}, 2 \mathrm{H}), 7.72(\mathrm{td}, J=8.2,5.3 \mathrm{~Hz}, 1 \mathrm{H})$, $7.48-7.43(\mathrm{~m}, 1 \mathrm{H}), 7.42-7.37(\mathrm{~m}, 2 \mathrm{H}), 7.20-7.12(\mathrm{~m}, 1 \mathrm{H}), 4.92(\mathrm{tt}, J=10.3,7.9 \mathrm{~Hz}, 1 \mathrm{H})$, $3.41-3.32(\mathrm{~m}, 1 \mathrm{H}), 2.25$ (ddd, $J=30.7,12.6,8.0 \mathrm{~Hz}, 2 \mathrm{H}), 2.11-2.11(\mathrm{~m}, 2 \mathrm{H}), 1.80-1.61(\mathrm{~m}$, $2 \mathrm{H}), 1.57-1.51(\mathrm{~m}, 2 \mathrm{H}), 1.02(\mathrm{dt}, J=9.3,3.2 \mathrm{~Hz}, 1 \mathrm{H}), 0.96(\mathrm{t}, J=7.4 \mathrm{~Hz}, 3 \mathrm{H})$.

Compound 34: 4-chloro-N-(1-((1R,3s,5S,6r)-3-(4-cyclopropyl-1H-1,2,3-triazol-1-yl)bicyclo [3.1.0]hexan-6-yl)propyl)benzamide
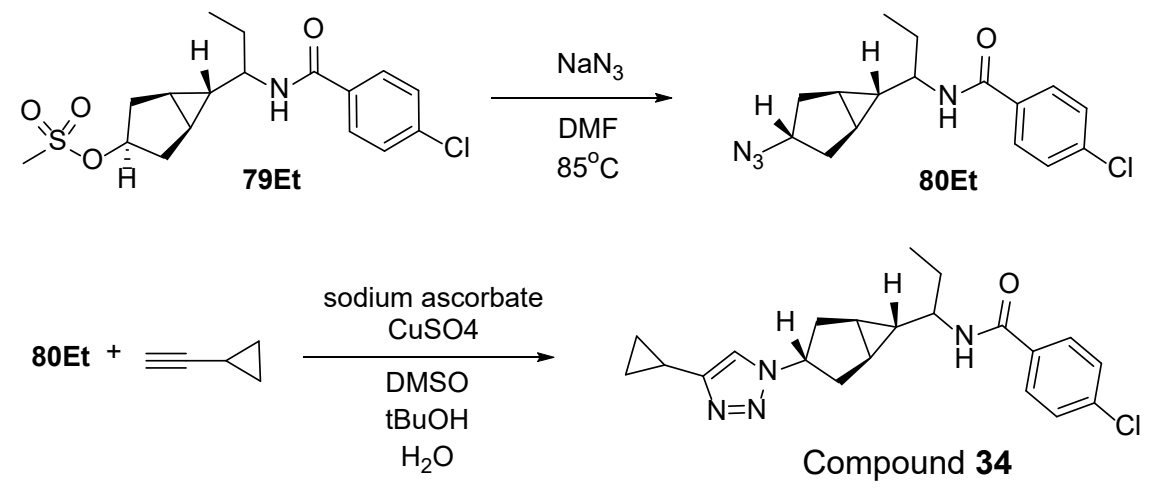

Compound 80: $N-(1-((1 R, 3 s, 5 S, 6 r)-3$-azidobicyclo[3.1.0] hexan-6-yl)propyl)-4-chlorobenzamide

To a solution of (1R,3r,5S,6r)-6-(1-(4-chlorobenzamido)propyl)bicyclo[3.1.0]hexan-3-yl methanesulfonate 79Et (100 mg, $0.27 \mathrm{mmol})$ in DMF (670 $\mu$ l) was added sodium azide (70 mg, $1.1 \mathrm{mmol})$ and the resulting mixture was stirred at $85^{\circ} \mathrm{C}$ for $1 \mathrm{~h}$. The reaction mixture was diluted with EtOAc $(50 \mathrm{~mL})$ and washed with $\mathrm{H}_{2} \mathrm{O}(50 \mathrm{~mL})$ and saturated $\mathrm{NaCl}(50 \mathrm{~mL})$, dried over $\mathrm{Na}_{2} \mathrm{SO}_{4}$, filtered, and concentrated to give the title compound $80 \mathrm{Et}$ (134 mg, $0.252 \mathrm{mmol}$, 94\%) that was used without further purification. $\mathrm{MS}\left(\mathrm{ES}^{+}\right) \mathrm{C}_{16} \mathrm{H}_{19} \mathrm{ClN}_{4} \mathrm{O}$ requires: 318 , found: $319[\mathrm{M}+\mathrm{H}]^{+}$.

Compound 34: 4-chloro-N-(1-((1R,3s,5S,6r)-3-(4-cyclopropyl-1H-1,2,3-triazol-1-yl)bicyclo [3.1.0]hexan-6-yl)propyl)benzamide. 
To a solution of N-(1-((1R,3s,5S,6r)-3-azidobicyclo[3.1.0]hexan-6-yl)propyl)-4chlorobenzamide 80Et (10 mg, $0.031 \mathrm{mmol})$ in DMSO (35 $\mu \mathrm{l})$ were added ethynylcyclopropane ( $2.5 \mathrm{mg}, 0.038 \mathrm{mmol}$ ), sodium ascorbate $(6 \mathrm{mg}, 0.031 \mathrm{mmol}$ ) dissolved in $40 \mu \mathrm{L}$ of water, copper(II) sulfate $(0.5 \mathrm{mg}, 3 \mu \mathrm{mol})$ dissolved in $10 \mu \mathrm{L}$ of water, and tBuOH (160 $\mu \mathrm{l})$ and the resulting mixture was stirred at room temperature for $72 \mathrm{~h}$. The residue was acidified with TFA and purified by mass-triggered preparative HPLC (Mobile phase: $\mathrm{A}=0.1 \% \mathrm{TFA} / \mathrm{H} 2 \mathrm{O}, \mathrm{B}=0.1 \%$ TFA/MeCN; Gradient: B = 30-70\%; 20 min; Column: C18) to give the title compound 34 (4 mg, $10 \mu \mathrm{mol}, 33 \%$ yield) as a white solid. $\mathrm{HRMS}\left(\mathrm{ES}^{+}\right) \mathrm{C}_{21} \mathrm{H}_{25} \mathrm{ClN}_{4} \mathrm{O}$ requires: $385.1790[\mathrm{M}+\mathrm{H}]^{+}$, found: $385.1786[\mathrm{M}+\mathrm{H}]^{+} .{ }^{1} \mathrm{H}$ NMR $\left(500 \mathrm{MHz}, \mathrm{CD}_{3} \mathrm{OD}\right) \delta 8.44(\mathrm{~d}, J=8.5 \mathrm{~Hz}, 1 \mathrm{H}), 7.81(\mathrm{~d}, J=$ $8.5 \mathrm{~Hz}, 2 \mathrm{H}), 7.72(\mathrm{~s}, 1 \mathrm{H}), 7.48(\mathrm{~d}, \mathrm{~J}=8.5 \mathrm{~Hz}, 2 \mathrm{H}), 4.55$ - $4.70(\mathrm{~m}, 1 \mathrm{H}), 3.30-3.9(\mathrm{~m}, 1 \mathrm{H}), 2.32$ $2.49(\mathrm{~m}, 2 \mathrm{H}), 2.18$ - $2.31(\mathrm{~m}, 2 \mathrm{H}), 1.87-1.97(\mathrm{~m}, 1 \mathrm{H}), 1.62$ - $1.81(\mathrm{~m}, 2 \mathrm{H}), 1.43-1.58(\mathrm{~m}, 2 \mathrm{H})$, $0.89-1.04(\mathrm{~m}, 6 \mathrm{H}), 0.70-0.77(\mathrm{~m}, 2 \mathrm{H})$.

Compound 35: 4-chloro-N-(1-((1R,3s,5S,6r)-3-(3-cyclopropyl-1H-1,2,4-triazol-1yl)bicyclo[3.1.0]hexan-6-yl)propyl)benzamide
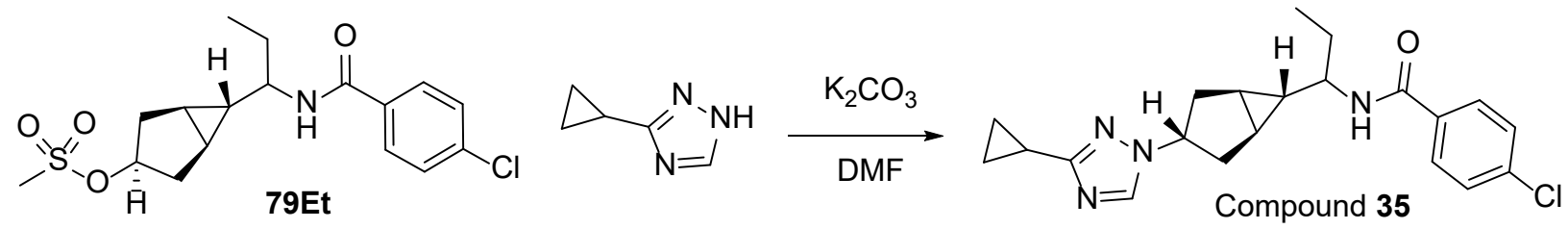

The title compound was synthesized similar to the mesylate displacement procedure (example 62) from (1R,3r,5S,6r)-6-(1-(4-chlorobenzamido)propyl)bicyclo[3.1.0]hexan-3-yl methanesulfonate (22 mg, $0.059 \mathrm{mmol}$ ), 3-cyclopropyl-1 H-1,2,4-triazole (7 mg, $0.07 \mathrm{mmol}$ ), and potassium carbonate $(25 \mathrm{mg}, 0.18 \mathrm{mmol})$ in DMF at $65^{\circ} \mathrm{C}$ to give $35(4.9 \mathrm{mg}, 0.013 \mathrm{mmol}, 21 \%$ yield) as an off-white solid. $\mathrm{MS}\left(\mathrm{ES}^{+}\right) \mathrm{C}_{21} \mathrm{H}_{25} \mathrm{ClN}_{4} \mathrm{O}$ requires: 384 , found: $385[\mathrm{M}+\mathrm{H}]^{+} .{ }^{1} \mathrm{H} \mathrm{NMR}$ $\left(600 \mathrm{MHz}, \mathrm{CD}_{3} \mathrm{OD}\right) \delta 8.58(\mathrm{~s}, 1 \mathrm{H}), 7.80(\mathrm{~d}, J=8.6 \mathrm{~Hz}, 2 \mathrm{H}), 7.48(\mathrm{~d}, J=8.4 \mathrm{~Hz}, 2 \mathrm{H}), 4.52-$ $4.43(\mathrm{~m}, 1 \mathrm{H}), 3.42-3.35(\mathrm{~m}, 1 \mathrm{H}), 2.40-2.33(\mathrm{~m}, 1 \mathrm{H}), 2.32-2.21(\mathrm{~m}, 3 \mathrm{H}), 2.02(\mathrm{tt}, J=8.5,4.9$ $\mathrm{Hz}, 1 \mathrm{H}), 1.81-1.63(\mathrm{~m}, 2 \mathrm{H}), 1.57-1.44(\mathrm{~m}, 2 \mathrm{H}), 1.04-1.00(\mathrm{~m}, 2 \mathrm{H}), 1.00-0.96(\mathrm{~m}, 4 \mathrm{H})$, $0.94-0.90(\mathrm{~m}, 2 \mathrm{H})$. 
Compound 36: N-(1-((1R,3s,5S,6r)-3-(1H-benzo[d]imidazol-1-yl)bicyclo[3.1.0]hexan-6yl)propyl)-4-chlorobenzamide

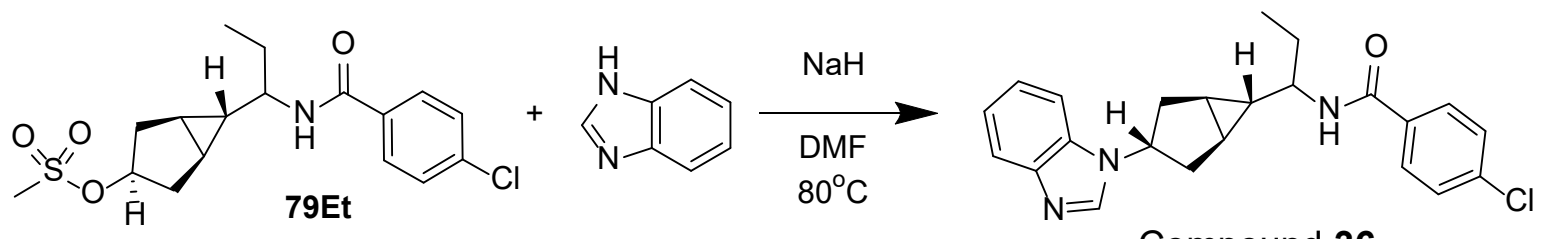

Compound 36

The title compound was synthesized similar to compound (62) by the General Mesylate displacement procedure from (1R,3r,5S,6r)-6-(1-(4chlorobenzamido)propyl)bicyclo[3.1.0]hexan-3-yl methanesulfonate and 1H-benzo[d]imidazole to give 24 as a white powder. $\mathrm{MS}\left(\mathrm{ES}^{+}\right) \mathrm{C}_{23} \mathrm{H}_{24} \mathrm{ClN}_{3} \mathrm{O}$ requires: 393 , found: $394[\mathrm{M}+\mathrm{H}]^{+}$. ${ }^{1} \mathrm{H} \mathrm{NMR}$ $\left(500 \mathrm{MHz}, \mathrm{CD}_{3} \mathrm{OD}\right) \delta 9.50(\mathrm{~s}, 1 \mathrm{H}), 8.45-8.50(\mathrm{~d}, \mathrm{~J}=8.5 \mathrm{~Hz}, 1 \mathrm{H}), 7.97(\mathrm{~m}, 1 \mathrm{H}), 7.75-7.87(\mathrm{~m}, 3 \mathrm{H})$, 7.62 - $7.71(\mathrm{~m}, 2 \mathrm{H}), 7.49$ (d, J = 8.5 Hz, 2H), 4.83 - $4.90(\mathrm{~m}, 1 \mathrm{H}), 3.40$ - $3.51(\mathrm{~m}, 1 \mathrm{H}), 2.52$ - 2.68 (m, 2H), $2.34-2.47(\mathrm{~m}, 2 \mathrm{H}), 1.71-1.85(\mathrm{~m}, 2 \mathrm{H}), 1.59-1.71(\mathrm{~m}, 2 \mathrm{H}), 1.14-1.20(\mathrm{~m}, 1 \mathrm{H}), 1.01$ $(\mathrm{t}, \mathrm{J}=7.3 \mathrm{~Hz}, 3 \mathrm{H})$.

Compound 37: 4-chloro-N-((S)-1-((1R,3R,5S,6r)-3-(4-fluoro-1H-benzo[d]imidazol-1yl)bicyclo[3.1.0]hexan-6-yl)propyl)benzamide

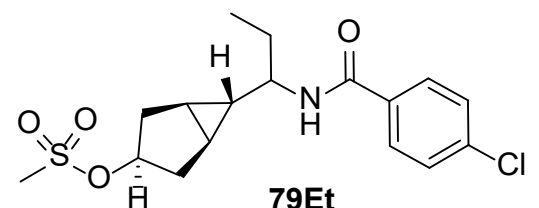

79Et<smiles>Fc1cccc2[nH]cnc12</smiles><smiles>CCC(NC(=O)c1ccc(Cl)cc1)C1[C@H]2CC(n3cnc4c(F)cccc43)C[C@H]12</smiles>

The title compound was synthesized similar to compound (35) by mesylate displacement procedure to give 37. MS ( $\left.\mathrm{ES}^{+}\right) \mathrm{C}_{23} \mathrm{H}_{23} \mathrm{ClFN}_{3} \mathrm{O}_{2}$ requires: 427, found: $428[\mathrm{M}+\mathrm{H}]^{+}{ }^{1} \mathrm{H}$ NMR (500 MHz, MeOD) $\delta 9.01(\mathrm{~s}, 1 \mathrm{H}), 7.85-7.79(\mathrm{~m}, 2 \mathrm{H}), 7.58(\mathrm{~d}, J=8.2 \mathrm{~Hz}, 1 \mathrm{H}), 7.51-7.41(\mathrm{~m}, 3 \mathrm{H})$, $7.31-7.24(\mathrm{~m}, 1 \mathrm{H}), 4.94-4.85(\mathrm{~m}, 1 \mathrm{H}), 3.44-3.32(\mathrm{~m}, 1 \mathrm{H}), 2.56-2.29(\mathrm{~m}, 4 \mathrm{H}), 1.84-1.66$ (m, 2H), $1.65-1.55(\mathrm{~m}, 2 \mathrm{H}), 1.10-1.03(\mathrm{~m}, 1 \mathrm{H}), 1.00(\mathrm{t}, J=7.4 \mathrm{~Hz}, 3 \mathrm{H})$.

Compound 38: 4-chloro-N-(1-((1R,3s,5S,6r)-3-(6-fluoro-1H-benzo[d]imidazol-1-yl)bicyclo [3.1.0]hexan-6-yl)propyl)benzamide. 

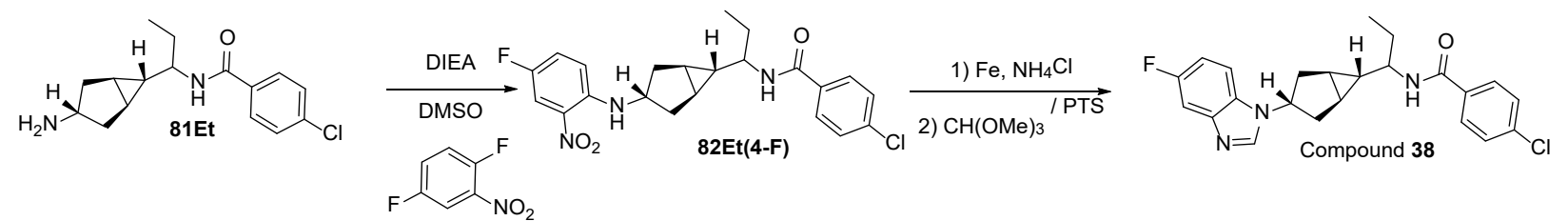

Compound

82Et(4-F):

4-chloro-N-(1-((1R,3s,5S,6r)-3-((4-fluoro-2nitrophenyl)amino)bicyclo[3.1.0]hexan-6-yl)propyl)benzamide.

N-(1-((1R,3s,5S,6r)-3-aminobicyclo[3.1.0]hexan-6-yl)propyl)-4-chlorobenzamide (369 mg, 1.26 mmol) was dissolved in DMSO $(12.6 \mathrm{~mL})$ and DIEA $(0.660 \mathrm{~mL}, 3.78 \mathrm{mmol})$ was added. 1,4difluoro-2-nitrobenzene $(0.143 \mathrm{ml}, 1.323 \mathrm{mmol})$ was added and the resulting mixture was stirred at $80^{\circ} \mathrm{C}$ overnight. The solution was washed with water, extracted with EtOAc, and the organics were concentrated under pressure. The residue was purified by Flash Chromatography ( $0-100 \%$ EtOAc/Hexanes) to give the title compound ( $461 \mathrm{mg}, 1.07 \mathrm{mmol}, 85 \%$ yield).

Step 1: $\quad N-(1-((1 R, 3 s, 5 S, 6 r)-3-((2$-amino-4-fluorophenyl)amino $)$ bicyclo[3.1.0]hexan-6yl)propyl)-4-chlorobenzamide.

4-chloro-N-((R)-1-((1R,3S,5S,6r)-3-((4-fluoro-2-nitrophenyl)amino)bicyclo[3.1.0]hexan-6yl)propyl)benzamide 82Et(4-F) (478 mg, $1.11 \mathrm{mmol}$ ) was dissolved in Ethanol $(8.3 \mathrm{~mL}) /$ Water $(2.8 \mathrm{~mL})$ and iron $(371 \mathrm{mg}, 6.64 \mathrm{mmol})$ and ammonium chloride $(59 \mathrm{mg}, 1.11 \mathrm{mmol})$ were added. The solution was heated to reflux for 2 hours. The reaction was filtered through Celite, concentrated under pressure to give the title compound and taken forward without purification. MS $\left(\mathrm{ES}^{+}\right) \mathrm{C}_{22} \mathrm{H}_{25} \mathrm{ClFN}_{3} \mathrm{O}$ requires 401, found $402[\mathrm{M}+\mathrm{H}]^{+}$.

Step 2 (Compound 38): 4-chloro-N-(1-((1R,3s,5S,6r)-3-(6-fluoro-1H-benzo[d]imidazol-1yl)bicyclo [3.1.0]hexan-6-yl)propyl)benzamide.

To a solution of N-(1-((1R,3s,5S,6r)-3-((2-amino-4-fluorophenyl)amino)bicyclo[3.1.0]hexan-6yl)propyl)-4-chlorobenzamide (445 $\mathrm{mg}, 1.11 \mathrm{mmol})$ in toluene $(11 \mathrm{~mL})$ was added trimethylorthoformate $(0.13 \mathrm{~mL}, 1.2 \mathrm{mmol})$ and p-toluenesulfonic acid monohydrate $(21 \mathrm{mg}, 0.11$ $\mathrm{mmol}$ ). The solution was stirred at refluxed for $2 \mathrm{~h}$. The solution was cooled to room temperature, concentrated, and purified by flash chromatography $(0-20 \% \mathrm{MeOH}$ in $\mathrm{DCM})$ to give the title compound 38. $\mathrm{HRMS}\left(\mathrm{ES}^{+}\right) \mathrm{C}_{23} \mathrm{H}_{23} \mathrm{ClFN}_{3} \mathrm{O}$ requires $412.1586[\mathrm{M}+\mathrm{H}]^{+}$, found $412.1581[\mathrm{M}+\mathrm{H}]^{+}$. ${ }^{1} \mathrm{H}$ NMR (CD 3 -OD) $\delta 8.94$ (d, $\left.J=12 \mathrm{~Hz}, 2 \mathrm{H}\right), 8.40-8.30(\mathrm{~m}, 1 \mathrm{H}), 7.85-7.75(\mathrm{~m}, 3 \mathrm{H}), 7.47$ (m, 
2H), $7.29-7.26(\mathrm{~m}, 1 \mathrm{H}), 4.80-4.65(\mathrm{~m}, 1 \mathrm{H}), 3.48-3.35(\mathrm{~m}, 1 \mathrm{H}), 2.65-2.45(\mathrm{~m}, 2 \mathrm{H}), 2.40-2.30$ (m, 2H), $1.80-1.60(\mathrm{~m}, 4 \mathrm{H}), 1.20-1.10(\mathrm{~m}, 1 \mathrm{H}), 1.05-0.95(\mathrm{t}, J=12 \mathrm{~Hz}, 3 \mathrm{H})$.

Compound 39: 4-chloro-N-(1-((1R,3s,5S,6r)-3-(6-fluoro-1H-benzo[d]imidazol-1yl)bicyclo[3.1.0]hexan-6-yl)propyl)benzamide

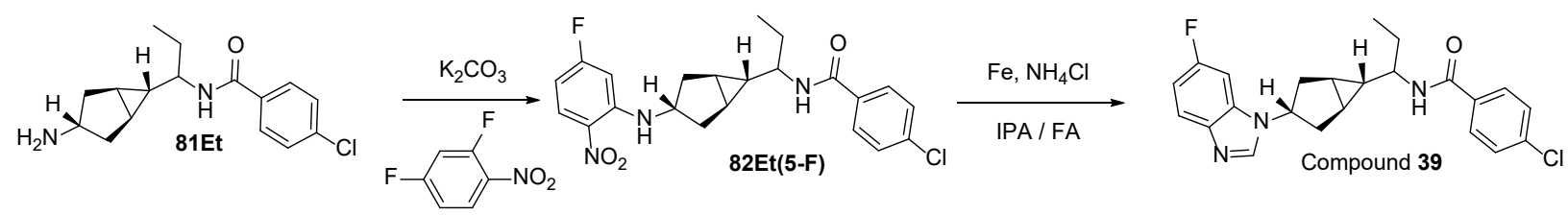

Compound $\quad$ 82Et(5-F): $\quad 4$-chloro- $N-((R)-1-((1 R, 3 S, 5 S, 6 r)-3-((5-$ fluoro-2nitrophenyl)amino)bicyclo[3.1.0]hexan-6-yl)propyl)benzamide.

To a cooled $0{ }^{\circ} \mathrm{C}$ solution of $\mathrm{N}-((\mathrm{R})-1-((1 \mathrm{R}, 3 \mathrm{~S}, 5 \mathrm{~S}, 6 \mathrm{r})-3$-aminobicyclo[3.1.0]hexan-6-yl)propyl)4-chlorobenzamide ( $330 \mathrm{mg}, 1.13 \mathrm{mmol}$ ) in THF ( $2.3 \mathrm{~mL}$ ) were added 2,4-difluoro-1-nitrobenzene (124 $\mu 1,1.13 \mathrm{mmol})$ and $\mathrm{K}_{2} \mathrm{CO}_{3}(156 \mathrm{mg}, 1.13 \mathrm{mmol})$. The resulting mixture was stirred at $25^{\circ} \mathrm{C}$ for $12 \mathrm{~h}$. The reaction mixture was filtered through celite, and the filtrate was concentrated under reduced pressure. The residue was purified by silica gel chromatography $(0-90 \%$ EtOAc in hexanes) to give (390 $\mathrm{mg}, 0.903 \mathrm{mmol}, 80 \%$ yield) as a yellow solid 82Et(5-F). MS (ES $\left.{ }^{+}\right)$ $\mathrm{C}_{22} \mathrm{H}_{23} \mathrm{ClFN}_{3} \mathrm{O}_{3}$ requires: 431, found: $432[\mathrm{M}+\mathrm{H}]^{+}$.

Compound 39: 4-chloro-N-((R)-1-((1R,3S,5S,6r)-3-(6-fluoro-1H-benzo[d]imidazol-1yl)bicyclo[3.1.0]hexan-6-yl)propyl)benzamide.

To a solution of 4-chloro-N-((R)-1-((1R,3S,5S,6r)-3-((5-fluoro-2nitrophenyl)amino)bicyclo[3.1.0]hexan-6-yl)propyl)benzamide 82ET(5-F) (390 mg, $0.903 \mathrm{mmol}$ ) in 2-Propanol $(4515 \mu \mathrm{l})$ were added iron $(504 \mathrm{mg}, 9.03 \mathrm{mmol})$, ammonium chloride ( $483 \mathrm{mg}, 9.03$ $\mathrm{mmol})$, and Formic Acid $(4.5 \mathrm{~mL})$ and the resulting mixture was stirred at $80^{\circ} \mathrm{C}$ for $1 \mathrm{~h}$. The reaction mixture was cooled to $0{ }^{\circ} \mathrm{C}$ and saturated $\mathrm{NaHCO}_{3}$ was added. The reaction mixture was diluted with EtOAc $(10 \mathrm{~mL})$ and the layers were separated. The aqueous phase was extracted with EtOAc $(3 \times 10 \mathrm{~mL})$, the combined organic layers were washed with saturated $\mathrm{NaCl}$, dried over $\mathrm{Na}_{2} \mathrm{SO}_{4}$, filtered and concentrated under reduced pressure. The residue was purified by silica gel chromatography (0-10\% $\mathrm{MeOH}$ in DCM with 1\% TEA) to give the title compound 39 (327 $\mathrm{mg}$, 
$0.794 \mathrm{mmol}, 88 \%$ yield) as a off-white solid. HRMS ( $\left(\mathrm{SS}^{+}\right) \mathrm{C}_{23} \mathrm{H}_{23} \mathrm{ClFN}_{3} \mathrm{O}$ requires: 412.1586 $[\mathrm{M}+\mathrm{H}]^{+}$, found: $412.1583[\mathrm{M}+\mathrm{H}]^{+} .{ }^{1} \mathrm{H}$ NMR (DMSO-d6) $\delta 8.30-8.40(\mathrm{~m}, 2 \mathrm{H}), 7.90(\mathrm{~d}, J=8.3$ $\mathrm{Hz}, 2 \mathrm{H}), 7.63(\mathrm{dd}, J=8.7,4.9 \mathrm{~Hz}, 1 \mathrm{H}), 7.58-7.50(\mathrm{~m}, 3 \mathrm{H}), 7.07-7.01(\mathrm{~m}, 1 \mathrm{H}), 4.65-4.56(\mathrm{~m}$, 1H), $3.46-3.38(\mathrm{~m}, 1 \mathrm{H}), 2.38-2.13(\mathrm{~m}, 4 \mathrm{H}), 1.72-1.61(\mathrm{~m}, 2 \mathrm{H}), 1.52-1.37(\mathrm{~m}, 2 \mathrm{H}), 1.18-1.11$ (m, 1H), $0.93(\mathrm{t}, J=7.4 \mathrm{~Hz}, 3 \mathrm{H})$.

Compound 39A: 4-chloro-N-((R)-1-((1R,3S,5S,6r)-3-(6-fluoro-1H-benzo[d]imidazol-1yl)bicyclo[3.1.0]hexan-6-yl)propyl)benzamide.

Compound 39B: 4-chloro-N-((S)-1-((1R,3R,5S,6r)-3-(6-fluoro-1H-benzo[d]imidazol-1yl)bicyclo[3.1.0]hexan-6-yl)propyl)benzamide.
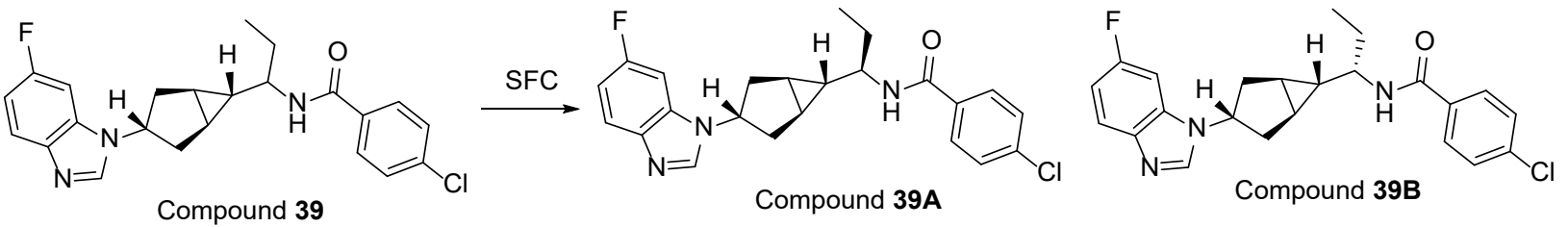

Enantiomers of the title compound 39 were separated by preparative SFC using the following conditions: column: 2.1 x $25.0 \mathrm{~cm}$ Chiralcel OX-H from Chiral Technologies (West Chester, PA); $\mathrm{CO}_{2}$ co-solvent (Solvent B): Ethanol with $0.25 \%$ Isopropylamine; isocratic method $35 \%$ cosolvent at $80 \mathrm{~g} / \mathrm{min}$; system pressure $120 \mathrm{bar}$; column temperature $25^{\circ} \mathrm{C}$; sample diluent Methanol; to give 39A as the first eluted product as a white solid (Averica Discovery Services, 50 D'Angelo Drive Suite 6 Marlborough MA 01752, AV17177 E1). HRMS (ES+) $\mathrm{C}_{23} \mathrm{H}_{23} \mathrm{ClFN}_{3} \mathrm{O}$ requires $412.1586[\mathrm{M}+\mathrm{H}]^{+}$, found $412.1579[\mathrm{M}+\mathrm{H}]^{+} .{ }^{1} \mathrm{H}$ NMR (DMSO-d6) $\delta: 8.30-8.40(\mathrm{~m}, 2 \mathrm{H}), 7.90(\mathrm{~d}$, $\mathrm{J}=8.3 \mathrm{~Hz}, 2 \mathrm{H}), 7.63(\mathrm{dd}, \mathrm{J}=8.7,4.9 \mathrm{~Hz}, 1 \mathrm{H}), 7.50-7.58(\mathrm{~m}, 3 \mathrm{H}), 7.01-7.07(\mathrm{~m}, 1 \mathrm{H}), 4.56-$ $4.650(\mathrm{~m}, 1 \mathrm{H}), 3.38$ - $3.46(\mathrm{~m}, 1 \mathrm{H}), 2.13-2.38(\mathrm{~m}, 4 \mathrm{H}), 1.61-7.72(\mathrm{~m}, 2 \mathrm{H}), 1.37$ - $1.52(\mathrm{~m}, 2 \mathrm{H})$, $1.11-1.18(\mathrm{~m}, 1 \mathrm{H}), 0.93(\mathrm{t}, \mathrm{J}=7.4 \mathrm{~Hz}, 3 \mathrm{H})$. The second eluted product, 39B as a white solid. HRMS (ES+) $\mathrm{C}_{23} \mathrm{H}_{23} \mathrm{ClFN}_{3} \mathrm{O}$ requires $412.1586[\mathrm{M}+\mathrm{H}]^{+}$, found $412.1581[\mathrm{M}+\mathrm{H}]^{+}$.

Compound 40: 4-chloro-N-((S)-1-((1R,3R,5S,6r)-3-(7-fluoro-1H-benzo[d]imidazol-1yl)bicyclo[3.1.0]hexan-6-yl)propyl)benzamide 

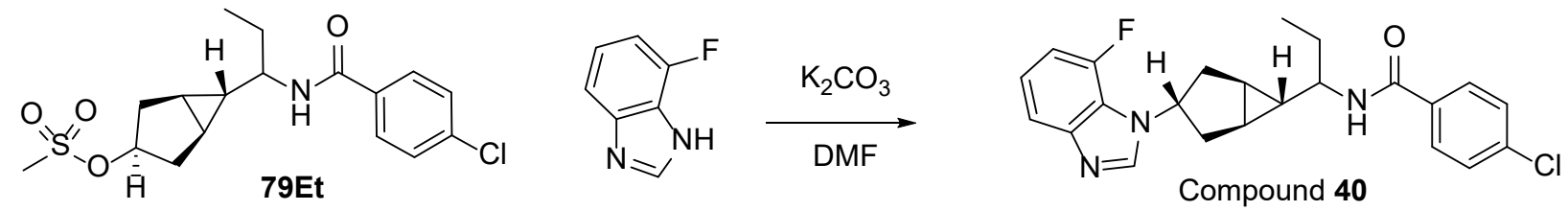

The title compound was synthesized similar to compound (35) by mesylate displacement procedure to give 40. $\mathrm{MS}\left(\mathrm{ES}^{+}\right) \mathrm{C}_{23} \mathrm{H}_{23} \mathrm{ClFN}_{3} \mathrm{O}$ requires: 411, found: $412[\mathrm{M}+\mathrm{H}]^{+}$.

${ }^{1} \mathrm{H}$ NMR (500 MHz, CD 3 OD) $\delta 9.01(\mathrm{~s}, 1 \mathrm{H}), 7.85-7.79(\mathrm{~m}, 2 \mathrm{H}), 7.58(\mathrm{~d}, J=8.2 \mathrm{~Hz}, 1 \mathrm{H}), 7.51$ - $7.41(\mathrm{~m}, 3 \mathrm{H}), 7.31-7.24(\mathrm{~m}, 1 \mathrm{H}), 4.94-4.85(\mathrm{~m}, 1 \mathrm{H}), 3.44-3.32(\mathrm{~m}, 1 \mathrm{H}), 2.56-2.29(\mathrm{~m}$, 4H), $1.84-1.66(\mathrm{~m}, 2 \mathrm{H}), 1.65-1.55(\mathrm{~m}, 2 \mathrm{H}), 1.10-1.03(\mathrm{~m}, 1 \mathrm{H}), 1.00(\mathrm{t}, J=7.4 \mathrm{~Hz}, 3 \mathrm{H})$.

Compound 41: 4-chloro-N-(1-((1R,3s,5S,6r)-3-(5-cyano-1H-benzo[d]imidazol-1yl)bicyclo[3.1.0]hexan-6-yl)propyl)benzamide<smiles>[R16]O[Na]</smiles><smiles>CCC(NC(=O)c1ccc(Cl)cc1)C(CC)NC(=O)c1ccc(Cl)cc1</smiles>

Compound 81Et: $N(1$ ((1R,3s, 5S,6r)-3-aminobicyclo[3.1.0]hexan-6-yl)propyl)-4chlorobenzamide

To a solution of N-(1-((1R,3s,5S,6r)-3-azidobicyclo[3.1.0]hexan-6-yl)propyl)-4chlorobenzamide 80Et $(1 \mathrm{~g}, 3.1 \mathrm{~mol})$ in THF $(9.5 \mathrm{~mL})$ and water $(0.95 \mathrm{~mL})$ was added triphenylphosphine ( $2.47 \mathrm{~g}, 9.41 \mathrm{mmol}$ ). The solution was heated to $55^{\circ} \mathrm{C}$ for $1.5 \mathrm{~h}$. The solution was concentrated under reduced pressure and the residue was purified by column chromatography (isocratic 20\% $\mathrm{MeOH} / 1 \%$ triethylamine / $79 \% \mathrm{DCM}$ ) to the title compound 81Et (893 mg, 3.05 mmol, 97\% yield). $\mathrm{MS}\left(\mathrm{ES}^{+}\right) \mathrm{C}_{16} \mathrm{H}_{21} \mathrm{ClN}_{2} \mathrm{O}$ requires 292, found $293[\mathrm{M}+\mathrm{H}]^{+}$. 
Compound

82Et(4-CN):

4-chloro-N-(1-((1R,3s,5S,6r)-3-((4-cyano-2-

nitrophenyl)amino)bicyclo[3.1.0]hexan-6-yl)propyl)benzamide

To a solution of N-(1-((1R,3s,5S,6r)-3-aminobicyclo[3.1.0]hexan-6-yl)propyl)-4chlorobenzamide 81Et (50 mg, $0.17 \mathrm{mmol})$ in THF (350 mL) were added 4-fluoro-3nitrobenzonitrile $(28 \mathrm{mg}, 0.17 \mathrm{mmol})$ and $\mathrm{K}_{2} \mathrm{CO}_{3}(47 \mathrm{mg}, 0.34 \mathrm{mmol})$ and the resulting mixture was stirred at room temperature overnight. The reaction was diluted with DCM, filtered over celite, concentrated, dissolved in minimal DCM, and purified by flash chromatography (10 - 60\% EtOAc in hexanes,) to give the title compound 82Et(4-CN) (59 mg, $0.14 \mathrm{mmol}, 79 \%$ yield) as a yellow solid. $\mathrm{MS}\left(\mathrm{ES}^{+}\right) \mathrm{C}_{23} \mathrm{H}_{23} \mathrm{ClN}_{4} \mathrm{O}_{3}$ requires: 438, found: $439[\mathrm{M}+\mathrm{H}]^{+}$.

Compound 41: 4-chloro-N-(1-((1R,3s,5S,6r)-3-(5-cyano-1H-benzo[d]imidazol-1yl)bicyclo[3.1.0]hexan-6-yl)propyl)benzamide

To a solution of 4-chloro-N-(1-((1R,3s,5S,6r)-3-((4-cyano-2-

nitrophenyl)amino)bicyclo[3.1.0]hexan-6-yl)propyl)benzamide 82Et(4-CN) in 2-propanol (350 $\mathrm{mL})$ and FA $(350 \mathrm{~mL})$ were added iron $(95 \mathrm{mg}, 1.7 \mathrm{mmol})$ and ammonium chloride (91 mg, 1.7 mmol)and the resulting mixture was stirred at $80{ }^{\circ} \mathrm{C}$ for $6 \mathrm{~h}$. The reaction was concentrated, diluted with DCM and 0.5 M NaOH. The mixture was filtered and the filtrate was separated. The aqueous layer was extracted with DCM $(3 \mathrm{x})$. The organic layers were combined, washed with saturated $\mathrm{NaCl}$, dried over $\mathrm{Na}_{2} \mathrm{SO}_{4}$, filtered, concentrated, and purified by flash chromatography (5-80\% of 80:20 EA:IPA in hexanes) to give the title compound 41 (35 mg, $0.083 \mathrm{mmol}, 48 \%$ yield) as a off-white solid. $\mathrm{MS}\left(\mathrm{ES}^{+}\right) \mathrm{C}_{24} \mathrm{H}_{23} \mathrm{ClN}_{4} \mathrm{O}$ requires: 418 , found: 419 $[\mathrm{M}+\mathrm{H}]^{+} .{ }^{1} \mathrm{H}$ NMR $(600 \mathrm{MHz}, \mathrm{DMSO}) \delta 8.61(\mathrm{~s}, 1 \mathrm{H}), 8.35(\mathrm{~d}, J=8.4 \mathrm{~Hz}, 1 \mathrm{H}), 8.20 \mathrm{f}(\mathrm{d}, J=1.5$ $\mathrm{Hz}, 1 \mathrm{H}), 7.90(\mathrm{~d}, J=8.6 \mathrm{~Hz}, 2 \mathrm{H}), 7.86(\mathrm{~d}, J=8.5 \mathrm{~Hz}, 1 \mathrm{H}), 7.65(\mathrm{dd}, J=8.5,1.6 \mathrm{~Hz}, 1 \mathrm{H}), 7.55$ $(\mathrm{d}, J=8.5 \mathrm{~Hz}, 2 \mathrm{H}), 4.76-4.66(\mathrm{~m}, 1 \mathrm{H}), 3.47-3.37$ (m, 1H), $2.41-2.34(\mathrm{~m}, 1 \mathrm{H}), 2.31-2.18$ $(\mathrm{m}, 3 \mathrm{H}), 1.72-1.60(\mathrm{~m}, 2 \mathrm{H}), 1.55-1.40(\mathrm{~m}, 2 \mathrm{H}), 1.15(\mathrm{dt}, J=8.5,3.2 \mathrm{~Hz}, 1 \mathrm{H}), 0.92(\mathrm{t}, J=7.4$ $\mathrm{Hz}, 3 \mathrm{H})$.

Compound 42: 4-chloro-N-(1-((1R,3s,5S,6r)-3-(4,6-difluoro-1H-benzo[d]imidazol-1yl)bicyclo[3.1.0]hexan-6-yl)propyl)benzamide. 


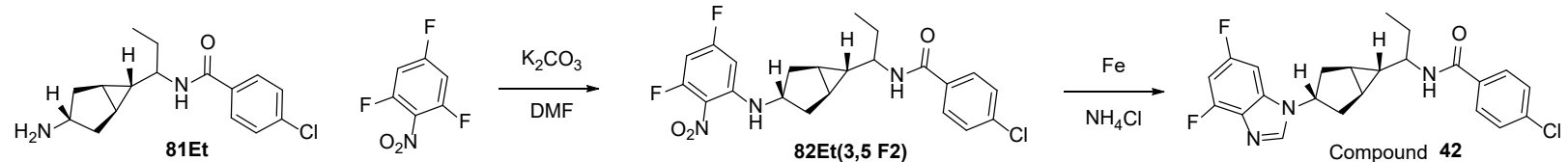

The title compound was synthesized similar to compound 41 from $\mathbf{8 1 E t}$ (30 $\mathrm{mg}, 0.10 \mathrm{mmol}$ ), 1,3,5-trifluoro-2-nitrobenzene (19 mg, $0.10 \mathrm{mmol})$, and $\mathrm{K}_{2} \mathrm{CO}_{3}(28 \mathrm{mg}, 0.21 \mathrm{mmol})$ in THF to give intermediate compound $\mathbf{8 2 E t}\left(3,5 \mathbf{F}_{2}\right)$ that was converted to the benzimidazole with iron (37 $\mathrm{mg}, 0.67 \mathrm{mmol})$ and $\mathrm{NH}_{4} \mathrm{Cl}(35 \mathrm{mg}, 0.67 \mathrm{mmol})$ in IPA:FA $(1: 1,0.66 \mathrm{~mL})$ to give $42(8.7 \mathrm{mg}$, 0.020 mmol, $30 \%$ yield) as a white solid. $\mathrm{MS}\left(\mathrm{ES}^{+}\right) \mathrm{C}_{23} \mathrm{H}_{22} \mathrm{ClF}_{2} \mathrm{~N}_{3} \mathrm{O}$ requires: 429 , found: 430 $[\mathrm{M}+\mathrm{H}]^{+} .{ }^{1} \mathrm{H}$ NMR $\left(600 \mathrm{MHz}, \mathrm{DMSO}-d_{6}\right) \delta 8.42(\mathrm{~s}, 1 \mathrm{H}), 8.32(\mathrm{~d}, J=8.4 \mathrm{~Hz}, 1 \mathrm{H}), 7.89(\mathrm{~d}, J=8.4$ $\mathrm{Hz}, 2 \mathrm{H}), 7.55(\mathrm{~s}, 2 \mathrm{H}), 7.48(\mathrm{dd}, J=9.3,2.2 \mathrm{~Hz}, 1 \mathrm{H}), 7.10-7.02(\mathrm{~m}, 1 \mathrm{H}), 4.65-4.56(\mathrm{~m}, 1 \mathrm{H})$, $3.46-3.37(\mathrm{~m}, 1 \mathrm{H}), 2.38-2.32(\mathrm{~m}, 1 \mathrm{H}), 2.29-2.14(\mathrm{~m}, 3 \mathrm{H}), 1.72-1.59(\mathrm{~m}, 2 \mathrm{H}), 1.53-1.37$ $(\mathrm{m}, 2 \mathrm{H}), 1.14(\mathrm{dt}, J=8.5,3.3 \mathrm{~Hz}, 1 \mathrm{H}), 0.92(\mathrm{t}, J=7.4 \mathrm{~Hz}, 3 \mathrm{H})$.

Compound 43: 4-chloro-N-(1-((1R,3s,5S,6r)-3-(5,7-difluoro-1H-benzo[d]imidazol-1yl)bicyclo[3.1.0]hexan-6-yl)propyl)benzamide
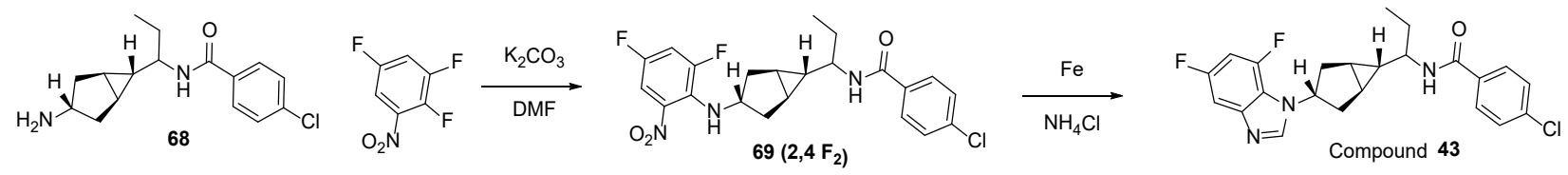

The title compound was synthesized similar to compound 41 from $\mathbf{8 1 E t}$ (50 $\mathrm{mg}, 0.17 \mathrm{mmol}$ ), 1,2,5-trifluoro-2-nitrobenzene (30 mg, $0.17 \mathrm{mmol})$, and $\mathrm{K}_{2} \mathrm{CO}_{3}(47 \mathrm{mg}, 0.34 \mathrm{mmol})$ in THF (0.3 $\mathrm{mL})$ to give intermediate compound $\mathbf{8 2 E t}\left(\mathbf{2}, \mathbf{4 5} \mathbf{F}_{2}\right)(65 \mathrm{mg}, 0.15 \mathrm{mmol})$ that was converted to the benzimidazole with iron $(95 \mathrm{mg}, 1.7 \mathrm{mmol})$ and $\mathrm{NH}_{4} \mathrm{Cl}(91 \mathrm{mg}, 1.7 \mathrm{mmol})$ in IPA:FA $(1: 1,0.66$ $\mathrm{mL}$ ) to give 43 (29 mg, $0.067 \mathrm{mmol}, 39 \%$ yield) as an off-white solid. MS (ES ${ }^{+}$) $\mathrm{C}_{23} \mathrm{H}_{22} \mathrm{ClF}_{2} \mathrm{~N}_{3} \mathrm{O}$ requires: 429 , found: $430[\mathrm{M}+\mathrm{H}]^{+}$. ${ }^{1} \mathrm{H}$ NMR $\left(600 \mathrm{MHz}, \mathrm{DMSO}-d_{6}\right) \delta 8.43$ (s, $1 \mathrm{H}), 8.35(\mathrm{~d}, J=8.6 \mathrm{~Hz}, 1 \mathrm{H}), 7.89(\mathrm{~d}, J=8.6 \mathrm{~Hz}, 2 \mathrm{H}), 7.54(\mathrm{~d}, J=8.5 \mathrm{~Hz}, 2 \mathrm{H}), 7.35(\mathrm{dd}, J=$ 9.3, $2.3 \mathrm{~Hz}, 1 \mathrm{H}), 7.20-7.13(\mathrm{~m}, 1 \mathrm{H}), 4.77-4.67(\mathrm{~m}, 1 \mathrm{H}), 3.41-3.33(\mathrm{~m}, 1 \mathrm{H}), 2.33(\mathrm{dd}, J=$ $12.8,7.7 \mathrm{~Hz}, 1 \mathrm{H}), 2.29-2.22(\mathrm{~m}, 2 \mathrm{H}), 2.20-2.13(\mathrm{~m}, 1 \mathrm{H}), 1.70-1.57(\mathrm{~m}, 2 \mathrm{H}), 1.49-1.41(\mathrm{~m}$, 2H), $0.99(\mathrm{dt}, J=8.8,3.3 \mathrm{~Hz}, 1 \mathrm{H}), 0.90(\mathrm{t}, J=7.4 \mathrm{~Hz}, 3 \mathrm{H})$. 
Compound 44: Synthesis of 4-chloro-N-(1-((1R,3s,5S,6r)-3-(6,7-difluoro-1Hbenzo[d]imidazol-1-yl)bicyclo [3.1.0]hexan-6-yl)propyl)benzamide.

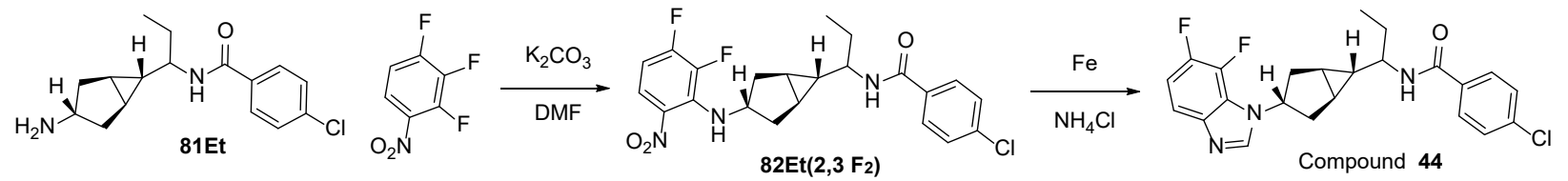

Compound $\quad \mathbf{8 2 E t}\left(2,3 \quad \mathbf{F}_{2}\right): \quad$ 4-chloro- $N-(1-((1 R, 3 s, 5 S, 6 r)-3-((2,3-$ difluoro-6nitrophenyl)amino)bicyclo[3.1.0]hexan-6-yl)propyl)benzamide

To a solution of 81Et (30 mg, $0.10 \mathrm{mmol})$ in THF $(200 \mu \mathrm{l})$ were added $\mathrm{K}_{2} \mathrm{CO}_{3}(28 \mathrm{mg}, 0.21 \mathrm{mmol})$ and 1,2,3-trifluoro-4-nitrobenzene $(19 \mathrm{mg}, 0.11 \mathrm{mmol})$. The reaction was stirred at room temperature for $1 \mathrm{~h}$, THF $(200 \mu \mathrm{l})$ was added, and stirring continued overnight. The reaction was diluted with water, extracted with ethyl acetate $(2 \mathrm{x})$, the organic layers washed with brine, combined, dried over $\mathrm{MgSO}_{4}$, filtered, concentrated, and purified by flash chromatography on silica gel (0-100\% EtOAc in hexanes) to give the title compound $\mathbf{8 2 E t}(2,3 \mathbf{~ F})$ (33 $\mathrm{mg}, 0.073$ mmol, $71.6 \%$ yield) as a yellow solid. MS (ES+) C22H22ClF2N3O3 requires: 449, found: 450 $[\mathrm{M}+\mathrm{H}]^{+}$.

Compound 44: 4-chloro-N-(1-((1R,3s,5S,6r)-3-(6,7-difluoro-1H-benzo[d]imidazol-1yl)bicyclo[3.1.0]hexan-6-yl)propyl)benzamide.

General one step synthesis of benzimidazoles. To a suspension of 4-chloro-N-(1-((1R,3s,5S,6r)3-((2,3-difluoro-6-nitrophenyl)amino) bicyclo[3.1.0]hexan-6-yl)propyl)benzamide 82Et(2,3 F2) (28 mg, $0.062 \mathrm{mmol})$ in 2-propanol $(311 \mu \mathrm{l})$ and formic Acid $(311 \mu \mathrm{l})$ were added $\mathrm{NH}_{4} \mathrm{Cl}(33$ $\mathrm{mg}, 0.62 \mathrm{mmol})$ and iron $(35 \mathrm{mg}, 0.62 \mathrm{mmol})$ and the resulting mixture was stirred at $80{ }^{\circ} \mathrm{C}$ for 6 h. The reaction was diluted with $\mathrm{DCM}$ and some $\mathrm{MeOH}$, filtered through celite, and the filtrate concentrated. The crude was diluted with DCM and washed with $0.2 \mathrm{M} \mathrm{NaOH}$ and brine. The aqueous layers were extracted with DCM $(3 \times 10 \mathrm{~mL})$. The organic layers were combined, dried over $\mathrm{Na}_{2} \mathrm{SO}_{4}$, filtered, concentrated, and purified by flash chromatography on silica gel (0-20\% of 8:2:1 DCM:MeOH:NH4OH in DCM) to give the title compound 44 (6 mg, $0.014 \mathrm{mmol}, 22 \%$ yield) as an off white solid. MS (ES $\left.{ }^{+}\right) \mathrm{C}_{23} \mathrm{H}_{22} \mathrm{ClF}_{2} \mathrm{~N}_{3} \mathrm{O}$ requires: 429 , found: $430[\mathrm{M}+\mathrm{H}]^{+}$. ${ }^{1} \mathrm{H}$ NMR (600 MHz, DMSO) $\delta 8.39(\mathrm{~s}, 1 \mathrm{H}), 8.36(\mathrm{~d}, J=8.4 \mathrm{~Hz}, 1 \mathrm{H}), 7.88(\mathrm{~d}, J=8.6 \mathrm{~Hz}, 1 \mathrm{H}), 7.55$ $(\mathrm{d}, J=8.6 \mathrm{~Hz}, 2 \mathrm{H}), 7.47(\mathrm{dd}, J=8.8,3.8 \mathrm{~Hz}, 1 \mathrm{H}), 7.30-7.22(\mathrm{~m}, 1 \mathrm{H}), 4.78-4.70(\mathrm{~m}, 1 \mathrm{H}), 3.36$ 
$(\mathrm{qd}, J=8.6,5.9 \mathrm{~Hz}, 1 \mathrm{H}), 2.38-2.31(\mathrm{~m}, 1 \mathrm{H}), 2.31-2.22(\mathrm{~m}, 2 \mathrm{H}), 2.22-2.15(\mathrm{~m}, 1 \mathrm{H}), 1.72-$ $1.58(\mathrm{~m}, 2 \mathrm{H}), 1.50-1.42(\mathrm{~m}, 2 \mathrm{H}), 0.99(\mathrm{dt}, J=8.7,3.3 \mathrm{~Hz}, 1 \mathrm{H}), 0.91(\mathrm{t}, J=7.4 \mathrm{~Hz}, 3 \mathrm{H})$.

Compound 45: 4-chloro-N-(1-((1R,3s,5S,6r)-3-(5,6-difluoro-1H-benzo[d]imidazol-1yl)bicyclo[3.1.0]hexan-6-yl)propyl)benzamide.
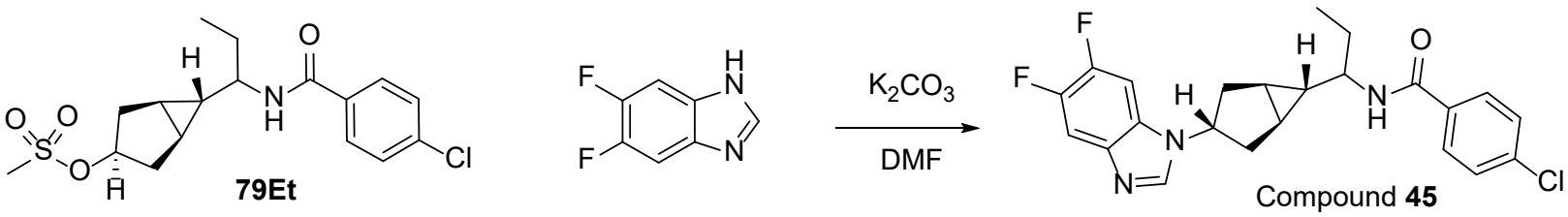

To a solution of (1R,3r,5S,6r)-6-(1-(4-chlorobenzamido)propyl)bicyclo[3.1.0]hexan-3-yl methanesulfonate 79Et (109 mg, $0.293 \mathrm{mmol})$ in DMF (980 $\mu \mathrm{l})$ were added 5,6-difluoro-1Hbenzo[d]imidazole $(60 \mathrm{mg}, 0.39 \mathrm{mmol})$ and $\mathrm{K}_{2} \mathrm{CO}_{3}(122 \mathrm{mg}, 0.879 \mathrm{mmol})$ and the resulting mixture was stirred at $65{ }^{\circ} \mathrm{C}$ for overnight. The reaction was diluted with $0.2 \mathrm{M} \mathrm{NaOH}$ and extracted with EtOAc $(2 \mathrm{x})$. The organic layers were washed with saturated $\mathrm{NaCl}$, combined, dried over $\mathrm{MgSO}_{4}$, concentrated as yellow film, and purified by flash chromatography ( 0 - $50 \%$ of 8:2 EtOAc:IPA in hexanes) to give the title compound 45 (36.5 mg, $0.085 \mathrm{mmol}, 29 \%$ yield).MS (ES $\left.{ }^{+}\right) \mathrm{C}_{2} 3 \mathrm{H}_{22} \mathrm{ClF}_{2} \mathrm{~N}_{3} \mathrm{O}$ requires: 429 , found: $430[\mathrm{M}+\mathrm{H}]^{+}$. ${ }^{1} \mathrm{H} \mathrm{NMR}(600 \mathrm{MHz}$, $\left.\mathrm{CD}_{3} \mathrm{OD}\right) \delta 8.29(\mathrm{~s}, 1 \mathrm{H}), 7.82(\mathrm{~d}, J=8.6 \mathrm{~Hz}, 2 \mathrm{H}), 7.54(\mathrm{dd}, J=10.4,7.0 \mathrm{~Hz}, 1 \mathrm{H}), 7.52-7.45(\mathrm{~m}$, $3 \mathrm{H}), 4.63-4.55(\mathrm{~m}, 1 \mathrm{H}), 3.46-3.38(\mathrm{~m}, 1 \mathrm{H}), 2.50-2.36(\mathrm{~m}, 2 \mathrm{H}), 2.35-2.25(\mathrm{~m}, 2 \mathrm{H}), 1.86-$ $1.66(\mathrm{~m}, 2 \mathrm{H}), 1.66-1.52(\mathrm{~m}, 2 \mathrm{H}), 1.15-1.09(\mathrm{~m}, 1 \mathrm{H}), 1.01(\mathrm{t}, J=7.4 \mathrm{~Hz}, 3 \mathrm{H})$.

Compound 46: 4-chloro-N-(1-((1R,3s,5S,6r)-3-(5,6-dichloro-1H-benzo[d]imidazol-1yl)bicyclo[3.1.0]hexan-6-yl)propyl)benzamide.

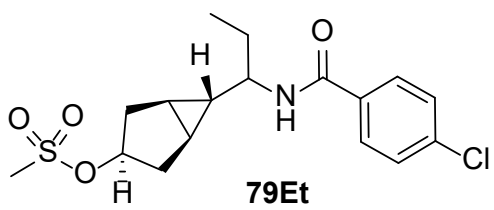<smiles>Clc1cc2nc[nH]c2cc1Cl</smiles>

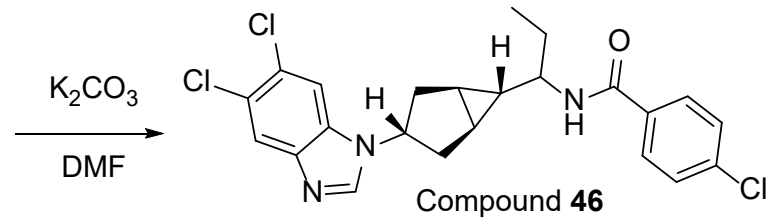

To a solution of (1R,3r,5S,6r)-6-(1-(4-chlorobenzamido)propyl)bicyclo[3.1.0]hexan-3-yl methanesulfonate 79Et (300 mg, $0.807 \mathrm{mmol}$ ) was dissolved in DMF (6 mL) was added $\mathrm{K}_{2} \mathrm{CO}_{3}$ (334 mg, $2.42 \mathrm{mmol}$ ) and 5,6-dichloro-1H-benzo[d]imidazole (166 mg, $0.887 \mathrm{mmol}$ ) and stirred overnight at $80^{\circ} \mathrm{C}$ for overnight. The reaction was diluted with water and extracted with EtOAc. The organics were collected, dried with $\mathrm{Na}_{2} \mathrm{SO}_{4}$, concentrated, and purified by flash 
chromatography (0-30\% of 80:20:1 DCM:MeOH:NH4OH in DCM) to give the title compound 46 (160 mg, $0.34 \mathrm{mmol}, 42 \%$ yield). The material was diluted in $\mathrm{ACN}$ and 1 eq. of $1 \mathrm{M} \mathrm{HCl}$ was added and the cloudy solution was lyophilized to give 4-chloro-N-((R)-1-((1R,3S,5S,6r)-3-(5,6dichloro-1H-benzo[d]imidazol-1-yl)bicyclo[3.1.0]hexan-6-yl)propyl)benzamide hydrochloride (150 mg, $0.31 \mathrm{mmol}, 38 \%$ yield) as an off white solid. HRMS (ES $\left.{ }^{+}\right) \mathrm{C}_{23} \mathrm{H}_{22} \mathrm{Cl}_{3} \mathrm{~N}_{3} \mathrm{O}$ requires: $462.0901[\mathrm{M}+\mathrm{H}]^{+}$, found: $462.0900[\mathrm{M}+\mathrm{H}]^{+} .{ }^{1} \mathrm{H}$ NMR (600 MHz, DMSO) $\delta 8.56(\mathrm{~s}, 1 \mathrm{H}), 8.31$ (d, $J=8.4 \mathrm{~Hz}, 1 \mathrm{H}), 8.09$ (s, 1H), $7.93(\mathrm{~s}, 1 \mathrm{H}), 7.89$ (d, $J=8.6 \mathrm{~Hz}, 2 \mathrm{H}), 7.55(\mathrm{~d}, J=8.5 \mathrm{~Hz}, 2 \mathrm{H})$, $4.71-4.61(\mathrm{~m}, 1 \mathrm{H}), 3.47-3.38(\mathrm{~m}, 1 \mathrm{H}), 2.41-2.33(\mathrm{~m}, 1 \mathrm{H}), 2.30-2.12(\mathrm{~m}, 3 \mathrm{H}), 1.72-1.60$ (m, 2H), $1.52-1.37$ (m, 2H), $1.16(\mathrm{dt}, J=8.3,3.3 \mathrm{~Hz}, 1 \mathrm{H}), 0.93(\mathrm{t}, J=7.4 \mathrm{~Hz}, 3 \mathrm{H})$.

Compound 47: N-(1-((1R,3s,5S,6r)-3-(1H-imidazo[4,5-b]pyridin-1-yl)bicyclo[3.1.0]hexan-6yl)propyl)-4-chlorobenzamide.

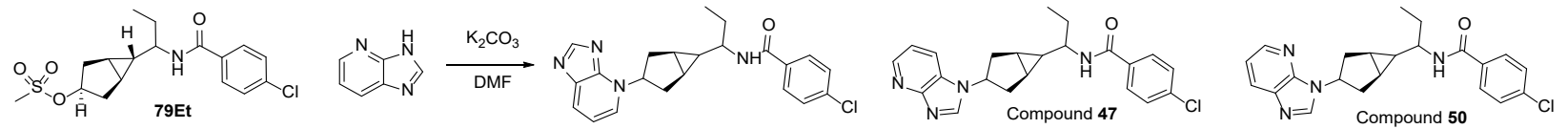

To a solution of (1R,3r,5S,6r)-6-(1-(4-chlorobenzamido)propyl)bicyclo[3.1.0]hexan-3-yl methanesulfonate 79Et (30 mg, $0.081 \mathrm{mmol})$ dissolved in DMF $(500 \mu \mathrm{L})$ was added $\mathrm{K}_{2} \mathrm{CO}_{3}(33$ $\mathrm{mg}, 0.24 \mathrm{mmol})$ and $3 \mathrm{H}$-imidazo[4,5-b]pyridine $(10.6 \mathrm{mg}, 0.089 \mathrm{mmol})$ and the solution was stirred overnight at $65^{\circ} \mathrm{C}$. The reaction was cooled in an ice bath acidified with TFA (75 uL), diluted with $\mathrm{MeOH}$ and purified by mass-triggered preparative HPLC (Mobile phase: $\mathrm{A}=0.1 \%$ TFA/H2O, B $=0.1 \%$ TFA/MeCN; Gradient: $\mathrm{B}=20-60 \% ; 20$ min; Column: $\mathrm{C} 18$ ) to give the title compound 47 as the second eluted product $(6.9 \mathrm{mg}, 0.017 \mathrm{mmol}, 21 \%$ yield) as a colorless amorphous material. MS (ES $\left.{ }^{+}\right) \mathrm{C}_{22} \mathrm{H}_{23} \mathrm{ClN} 4 \mathrm{O}$ requires: 394 , found: $395[\mathrm{M}+\mathrm{H}]^{+} .{ }^{1} \mathrm{H} \mathrm{NMR}(600$ $\left.\mathrm{MHz}, \mathrm{CD}_{3} \mathrm{OD}\right) \delta 9.11(\mathrm{~s}, 1 \mathrm{H}), 8.64(\mathrm{dd}, J=5.2,1.3 \mathrm{~Hz}, 1 \mathrm{H}), 8.51(\mathrm{dd}, J=8.3,1.4 \mathrm{~Hz}, 1 \mathrm{H}), 7.82$ $(\mathrm{d}, J=8.6 \mathrm{~Hz}, 2 \mathrm{H}), 7.65(\mathrm{dd}, J=8.3,5.2 \mathrm{~Hz}, 1 \mathrm{H}), 7.49$ (d, $J=8.6 \mathrm{~Hz}, 2 \mathrm{H}), 4.86-4.76(\mathrm{~m}, 1 \mathrm{H})$, $3.48-3.40(\mathrm{~m}, 1 \mathrm{H}), 2.63-2.48(\mathrm{~m}, 2 \mathrm{H}), 2.45-2.35(\mathrm{~m}, 2 \mathrm{H}), 1.86-1.72(\mathrm{~m}, 2 \mathrm{H}), 1.70-1.58$ (m, 2H), $1.15(\mathrm{dt}, J=9.0,3.3 \mathrm{~Hz}, 1 \mathrm{H}), 1.02(\mathrm{t}, J=7.4 \mathrm{~Hz}, 3 \mathrm{H})$.

Compound

48: N-((S)-1-((1R,3R,5S,6r)-3-(1H-imidazo[4,5-c]pyridin-1yl)bicyclo[3.1.0]hexan-6-yl)propyl)-4-chlorobenzamide. 

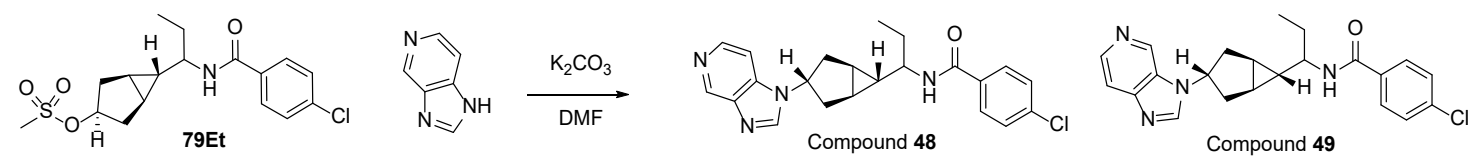

The title compound was synthesized similar to compound (35) by the mesylate displacement procedure to give $\mathbf{4 8}$ as the second eluting product from the mass-triggered preparative HPLC (Mobile phase: $\mathrm{A}=0.1 \% \mathrm{TFA} / \mathrm{H} 2 \mathrm{O}, \mathrm{B}=0.1 \% \mathrm{TFA} / \mathrm{MeCN}$; Gradient: $\mathrm{B}=20-60 \% ; 20 \mathrm{~min}$; Column: C18) purification. MS ( $\left.\mathrm{ES}^{+}\right) \mathrm{C}_{22} \mathrm{H}_{23} \mathrm{ClN}_{4} \mathrm{O}$ requires: 394, found: $395[\mathrm{M}+\mathrm{H}]^{+}$. ${ }^{1} \mathrm{H}$ NMR $(500 \mathrm{MHz}, \mathrm{MeOD}) \delta 9.21(\mathrm{~s}, 1 \mathrm{H}), 8.80(\mathrm{~s}, 1 \mathrm{H}), 8.50(\mathrm{~d}, J=6.5 \mathrm{~Hz}, 1 \mathrm{H}), 8.14(\mathrm{~d}, J=6.5 \mathrm{~Hz}, 1 \mathrm{H})$, $7.81-7.75(\mathrm{~m}, 2 \mathrm{H}), 7.47-7.41(\mathrm{~m}, 2 \mathrm{H}), 4.85-4.74(\mathrm{~m}, 1 \mathrm{H}), 3.43-3.35(\mathrm{~m}, 1 \mathrm{H}), 2.55-2.48$ (m, 1H), $2.48-2.41(\mathrm{~m}, 1 \mathrm{H}), 2.40-2.29(\mathrm{~m}, 2 \mathrm{H}), 1.82-1.70(\mathrm{~m}, 1 \mathrm{H}), 1.71-1.65(\mathrm{~m}, 1 \mathrm{H}), 1.65$

$-1.61(\mathrm{~m}, 1 \mathrm{H}), 1.59-1.52(\mathrm{~m}, 1 \mathrm{H}), 1.16-1.09(\mathrm{~m}, 1 \mathrm{H}), 0.97(\mathrm{t}, J=7.4 \mathrm{~Hz}, 3 \mathrm{H})$.

\section{Compound 49: N-((S)-1-((1R,3R,5S,6r)-3-(3H-imidazo[4,5-c]pyridin-3-}

\section{yl)bicyclo[3.1.0]hexan-6-yl)propyl)-4-chlorobenzamide.}

The title compound 49 was isolated as the first eluting product from the purification of $\mathbf{4 8}$. MS $\left(\mathrm{ES}^{+}\right) \mathrm{C}_{22} \mathrm{H}_{23} \mathrm{ClN}_{4} \mathrm{O}$ requires: 394, found: $395[\mathrm{M}+\mathrm{H}]^{+} .{ }^{1} \mathrm{H}$ NMR $(500 \mathrm{MHz}, \mathrm{MeOD}) \delta 9.06(\mathrm{~s}$, 1H), $8.62(\mathrm{~s}, 1 \mathrm{H}), 8.41$ - $8.37(\mathrm{~m}, 2 \mathrm{H}), 7.84-7.81(\mathrm{~m}, 3 \mathrm{H}), 7.47-7.41(\mathrm{~m}, 2 \mathrm{H}), 4.85$ - $4.74(\mathrm{~m}$, 1H), $3.43-3.35(\mathrm{~m}, 1 \mathrm{H}), 2.55-2.48(\mathrm{~m}, 1 \mathrm{H}), 2.48-2.41(\mathrm{~m}, 1 \mathrm{H}), 2.40-2.29(\mathrm{~m}, 2 \mathrm{H}), 1.82-$ $1.70(\mathrm{~m}, 1 \mathrm{H}), 1.71-1.65(\mathrm{~m}, 1 \mathrm{H}), 1.65-1.61(\mathrm{~m}, 1 \mathrm{H}), 1.59-1.52(\mathrm{~m}, 1 \mathrm{H}), 1.16-1.09(\mathrm{~m}, 1 \mathrm{H})$, $0.97(\mathrm{t}, J=7.4 \mathrm{~Hz}, 3 \mathrm{H})$.

Compound 50: N-(1-((1R,3s,5S,6r)-3-(3H-imidazo[4,5-b]pyridin-3-yl)bicyclo[3.1.0]hexan-6yl)propyl)-4-chlorobenzamide.

The title compound $\mathbf{5 0}$ was isolated as the third eluted product from the purification described for compound 47 (6.9 mg, $0.017 \mathrm{mmol}, 21 \%$ yield) as a colorless amorphous material. MS (ES $\left.{ }^{+}\right)$ $\mathrm{C}_{22} \mathrm{H}_{23} \mathrm{ClN}{ }_{4} \mathrm{O}$ requires: 394 , found: $395[\mathrm{M}+\mathrm{H}]^{+} .{ }^{1} \mathrm{H} \mathrm{NMR}\left(600 \mathrm{MHz}, \mathrm{CD}_{3} \mathrm{OD}\right) \delta 9.25(\mathrm{~s}, 1 \mathrm{H})$, $8.61(\mathrm{dd}, J=1.6,1.2 \mathrm{~Hz}, 1 \mathrm{H}), 8.21(\mathrm{dd}, J=8.3,1.4 \mathrm{~Hz}, 1 \mathrm{H}), 7.83(\mathrm{~d}, J=8.6 \mathrm{~Hz}, 2 \mathrm{H}), 7.56(\mathrm{dd}, J$ $=8.3,4.8 \mathrm{~Hz}, 1 \mathrm{H}), 7.48(\mathrm{~d}, J=8.4 \mathrm{~Hz}, 2 \mathrm{H}), 5.00-4.91(\mathrm{~m}, 1 \mathrm{H}), 3.46-3.39(\mathrm{~m}, 1 \mathrm{H}), 2.60-$ 
$2.45(\mathrm{~m}, 4 \mathrm{H}), 1.87-1.69(\mathrm{~m}, 2 \mathrm{H}), 1.68-1.56(\mathrm{~m}, 2 \mathrm{H}), 1.15(\mathrm{dt}, J=9.1,3.3 \mathrm{~Hz}, 1 \mathrm{H}), 1.01(\mathrm{t}, J=$ $7.5 \mathrm{~Hz}, 3 \mathrm{H})$.

Compound 51: 4-chloro-N-(1-((1R,3s,5S,6r)-3-(6-fluoro-3H-imidazo[4,5-b]pyridin-3yl)bicyclo[3.1.0]hexan-6-yl)propyl)benzamide.
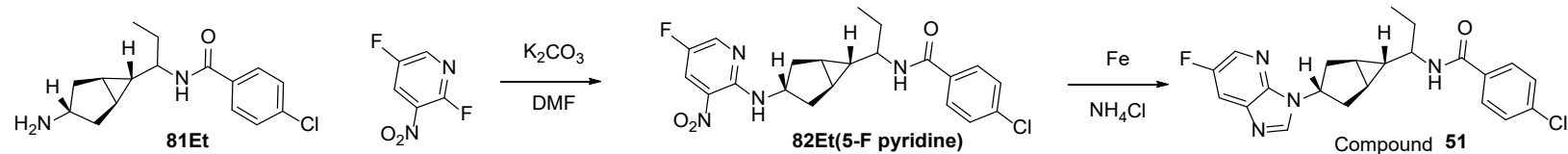

The title compound was synthesized similar to compound 41 from $81 E t$ (100 mg, 0.34 mmol), 2chloro-5-fluoro-3-nitropyridine (64 mg, $0.36 \mathrm{mmol})$ and DIEA (178 $\mu \mathrm{L}, 0.95 \mathrm{mmol})$ in DMSO (3 mL) to give intermediate compound 82Et(5-F, pyridine) (126 mg, $0.29 \mathrm{mmol}, 85 \%)$ that was converted to the benzimidazole with iron $(98 \mathrm{mg}, 1.7 \mathrm{mmol})$ and $\mathrm{NH}_{4} \mathrm{Cl}(92 \mathrm{mg}, 1.7 \mathrm{mmol})$ in EtOH: $\mathrm{H}_{2} \mathrm{O}: \mathrm{FA}(2: 0.5: 4 \mathrm{~mL})$ to give 51 (41 mg, $0.10 \mathrm{mmol}, 34 \%$ yield) as a white solid. MS $\left(\mathrm{ES}^{+}\right) \mathrm{C}_{22} \mathrm{H}_{22} \mathrm{ClFN}_{4} \mathrm{O}$ requires: 412, found: $413[\mathrm{M}+\mathrm{H}]^{+} .{ }^{1} \mathrm{H}$ NMR (600 MHz, DMSO-d6) $\delta 8.62$ (s, 1H), $8.40-8.38(\mathrm{~m}, 1 \mathrm{H}), 8.36(\mathrm{~d}, J=8.5 \mathrm{~Hz}, 1 \mathrm{H}), 8.05(\mathrm{dd}, J=9.4,2.7 \mathrm{~Hz}, 1 \mathrm{H}), 7.89(\mathrm{~d}, J=$ $8.6 \mathrm{~Hz}, 2 \mathrm{H}), 7.55(\mathrm{~d}, J=8.5 \mathrm{~Hz}, 2 \mathrm{H}), 4.77-4.66(\mathrm{~m}, 1 \mathrm{H}), 3.41-3.35(\mathrm{~m}, 1 \mathrm{H}), 2.47-2.33(\mathrm{~m}$, 2H), $2.32-2.19(\mathrm{~m}, 2 \mathrm{H}), 1.72-1.59(\mathrm{~m}, 2 \mathrm{H}), 1.48-1.42(\mathrm{~m}, 2 \mathrm{H}), 1.09(\mathrm{dt}, J=8.7,3.2 \mathrm{~Hz}$, $1 \mathrm{H}), 0.91(\mathrm{t}, J=7.4 \mathrm{~Hz}, 3 \mathrm{H})$.

Compound 52: 4-chloro-N-(1-((1R,3s,5S,6r)-3-(6-cyano-3H-imidazo[4,5-b]pyridin-3yl)bicyclo[3.1.0]hexan-6-yl)propyl)benzamide.
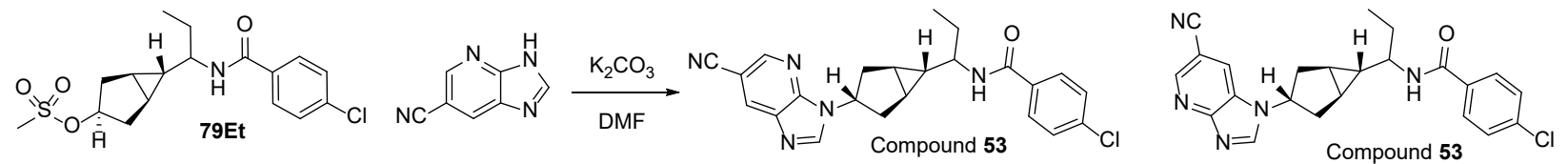

(1R,3r,5S,6r)-6-(1-(4-chlorobenzamido)propyl)bicyclo[3.1.0]hexan-3-yl methanesulfonate 79Et (40 mg, $0.11 \mathrm{mmol})$ was dissolved in DMF (1100 $\mu \mathrm{l})$ and $\mathrm{K}_{2} \mathrm{CO}_{3}(45 \mathrm{mg}, 0.32 \mathrm{mmol})$ along with 3H-imidazo[4,5-b]pyridine-6-carbonitrile $(17 \mathrm{mg}, 0.12 \mathrm{mmol})$ was added and stirred overnight at $55^{\circ} \mathrm{C}$. The reaction was diluted with water and extracted with EtOAc $(2 \mathrm{x})$. The organics washed with brine, combined, concentrated and purified by flash chromatography $(0-100 \%$, 80:20 EtOAc:IPA in hexanes to the title compound $\mathbf{5 2}$ as the second eluted product $(6.2 \mathrm{mg}$, 
0.015 mmol, $13 \%$ yield) as a white solid. $\mathrm{MS}\left(\mathrm{ES}^{+}\right) \mathrm{C}_{23} \mathrm{H}_{22} \mathrm{ClN}_{5} \mathrm{O}$ requires: 419 , found: 420 $[\mathrm{M}+\mathrm{H}]^{+} .{ }^{1} \mathrm{H}$ NMR $\left(500 \mathrm{MHz}, \mathrm{DMSO}-d_{6}\right) \delta 8.81(\mathrm{~s}, 1 \mathrm{H}), 8.80(\mathrm{~d}, J=1.8 \mathrm{~Hz}, 1 \mathrm{H}), 8.69(\mathrm{~d}, J=1.8$ $\mathrm{Hz}, 1 \mathrm{H}), 8.35$ (d, $J=8.4 \mathrm{~Hz}, 1 \mathrm{H}), 7.89$ (d, $J=8.6 \mathrm{~Hz}, 2 \mathrm{H}), 7.55$ (d, $J=8.6 \mathrm{~Hz}, 2 \mathrm{H}), 4.77$ (ddd, $J$ $=17.8,10.2,7.6 \mathrm{~Hz}, 1 \mathrm{H}), 3.41-3.34(\mathrm{~m}, 1 \mathrm{H}), 2.47-2.20(\mathrm{~m}, 4 \mathrm{H}), 1.71-1.58(\mathrm{~m}, 2 \mathrm{H}), 1.49-$ $1.44(\mathrm{~m}, 2 \mathrm{H}), 1.10(\mathrm{dt}, J=8.9,3.2 \mathrm{~Hz}, 1 \mathrm{H}), 0.91(\mathrm{t}, J=7.4 \mathrm{~Hz}, 3 \mathrm{H})$.

Compound 53: 4-chloro-N-(1-((1R,3s,5S,6r)-3-(6-cyano-1H-imidazo[4,5-b]pyridin-1yl)bicyclo[3.1.0]hexan-6-yl)propyl)benzamide.

The title compound $\mathbf{5 3}$ was isolated as the third eluted product during the purification described for compound 52 (1.9 mg, $4.52 \mu$ mol, $4.2 \%$ yield) as a white solids. $\mathrm{MS}\left(\mathrm{ES}^{+}\right) \mathrm{C}_{23} \mathrm{H}_{22} \mathrm{ClN}_{5} \mathrm{O}$ requires: 419, found: $420[\mathrm{M}+\mathrm{H}]^{+} .{ }^{1} \mathrm{H}$ NMR $\left(500 \mathrm{MHz}, \mathrm{CD}_{3} \mathrm{OD}\right) \delta 8.77-8.74(\mathrm{~m}, 2 \mathrm{H}), 8.56(\mathrm{~d}$, $J=1.9 \mathrm{~Hz}, 1 \mathrm{H}), 7.81(\mathrm{~d}, J=8.6 \mathrm{~Hz}, 2 \mathrm{H}), 7.48(\mathrm{~d}, J=8.6 \mathrm{~Hz}, 2 \mathrm{H}), 4.71(\mathrm{tt}, J=9.9,7.6 \mathrm{~Hz}, 1 \mathrm{H})$, $3.46-3.38(\mathrm{~m}, 1 \mathrm{H}), 2.57-2.41(\mathrm{~m}, 2 \mathrm{H}), 2.41-2.30(\mathrm{~m}, 2 \mathrm{H}), 1.86-1.68(\mathrm{~m}, 2 \mathrm{H}), 1.67-1.55$ (m, 2H), $1.13(\mathrm{dt}, J=8.9,3.2 \mathrm{~Hz}, 1 \mathrm{H}), 1.01(\mathrm{t}, J=7.4 \mathrm{~Hz}, 3 \mathrm{H})$.

Compound 54: Synthesis of 4-chloro-N-((1R,3s,5S,6r)-3-(5,6-difluoro-1Hbenzo[d]imidazol-1-yl)bicyclo[3.1.0]hexan-6-yl)methyl)benzamide.

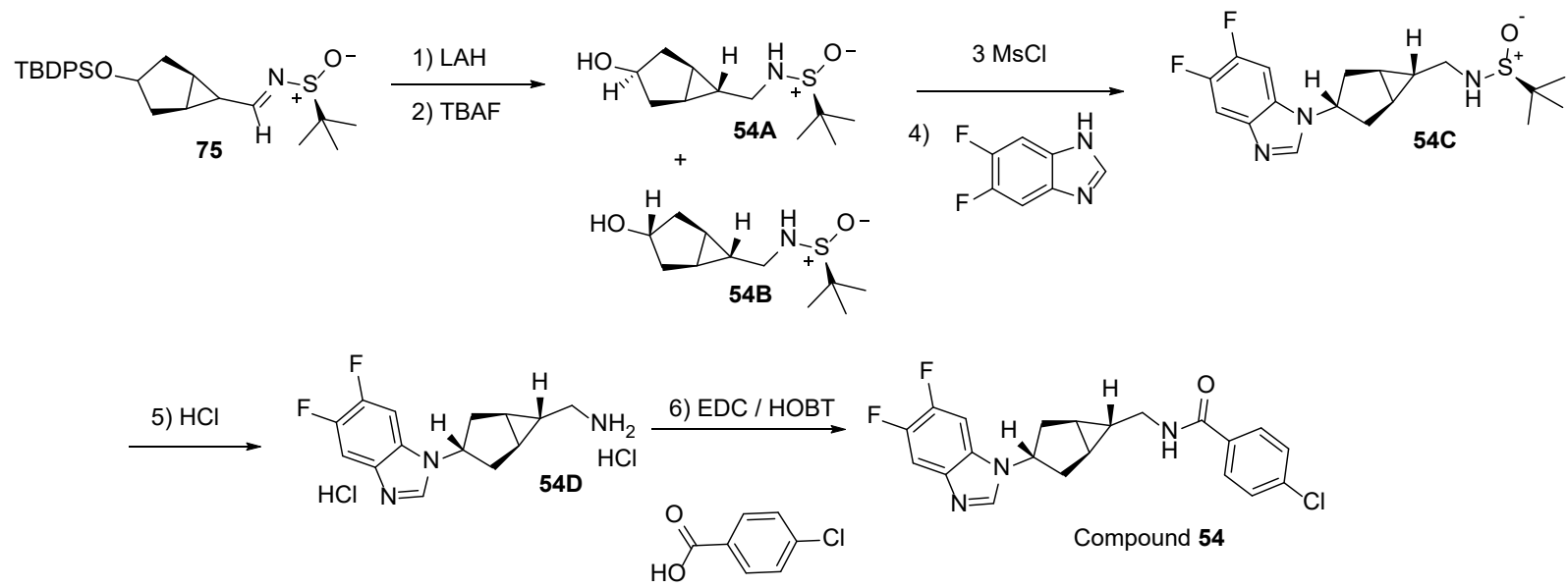

Step 1: (S)-N-(((1R,5S,6r)-3-((tert-butyldiphenylsilyl)oxy)bicyclo[3.1.0]hexan-6-yl)methyl)-2methylpropane-2-sulfinamide 54A-B(step1). To a solution of (S,E)-N-(((1R,5S)-3-((tertbutyldiphenylsilyl)oxy)bicyclo[3.1.0]hexan-6-yl)methylene)-2-methylpropane-2-sulfinamide 75 $(7.1 \mathrm{~g}, 15.1 \mathrm{mmol})$ in THF $(50 \mathrm{~mL})$ cooled in an acetone dry ice bath was added LAH $(11.3 \mathrm{~mL}$ 
of $2 \mathrm{M}$ THF solution, 22. mmol) and the resulting mixture was allowed to warm to RT overnight. The reaction was cooled in an ice bath and quenched following the Fieser and Fieser method (0.9 $\mathrm{mL}$ water, $0.9 \mathrm{~mL}$ of $15 \% \mathrm{NaOH}, 2.7 \mathrm{~mL}$ of water, added $\mathrm{MgSO} 4$, stirred for $30 \mathrm{~min}$, and filtered). The resulting filtrate was diluted with water and extracted with EtOAc. The organic layer was washed with brine, dried over $\mathrm{MgSO} 4$, filtered, and concentrated to give title compound 54A-B(step1) (6.35 g, 13.5 mmol, 89\% yield) as a clear semisolid. MS (ES+) $\mathrm{C}_{27} \mathrm{H}_{39} \mathrm{NO}_{2} \mathrm{SSi}$ requires: 469, found: $470[\mathrm{M}+\mathrm{H}]+$.

Step 2: (S)-N-(((1R,3S,5S,6r)-3-hydroxybicyclo[3.1.0]hexan-6-yl)methyl)-2-methylpropane-2sulfinamide 54A. (S)-N-(((1R,5S,6r)-3-((tert-butyldiphenylsilyl)oxy)bicyclo[3.1.0]hexan-6yl)methyl)-2-methylpropane-2-sulfinamide 54A(step1) (6.35 g, $13.52 \mathrm{mmol}$ ) was dissolved in THF $(50 \mathrm{~mL})$, cooled in an ice bath and TBAF (30.4 mL of 1M solution in THF, $30.4 \mathrm{mmol})$ was added dropwise. The reaction was removed from the bath and allowed to warm to RT overnight. The reaction was concentrated, diluted with water, and extracted twice with EtOAc. The organic layers were washed brine, combined, dried over MgSO4, filtered, concentrated, and purified by flash chromatography (10-10\% of 80:20 EtOAc:IPA in hexanes) to give both isomers.

The first isomer to elute was (S)-N-(((1R,3S,5S,6r)-3-hydroxybicyclo[3.1.0]hexan-6-yl)methyl)2-methylpropane-2-sulfinamide 54A (1.5 g, $6.5 \mathrm{mmol}, 42 \%$ yield $)$ which was obtained as a white solid. MS (ES $\left.{ }^{+}\right) \mathrm{C}_{11} \mathrm{H}_{21} \mathrm{NO} 2 \mathrm{~S}$ requires: 231, found: $232[\mathrm{M}+\mathrm{H}]^{+}$.

The second isomer to elute was (S)-N-(((1R,3R,5S,6r)-3-hydroxybicyclo[3.1.0]hexan-6yl)methyl)-2-methylpropane-2-sulfinamide 54B (0.3 g, $1.3 \mathrm{mmol}, 8.5 \%$ yield) which was obtained as white solid. MS (ES+) C11H21NO2S requires: 231, found: $232[\mathrm{M}+\mathrm{H}]+$.

Step 3: (1R,3S, 5S,6r)-6-(((S)-1,1-dimethylethylsulfinamido)methyl)bicyclo[3.1.0] hexan-3-yl methanesulfonate $\mathbf{5 4 C ( s t e p 3 ) . ~ T o ~ a ~ c o o l e d ~} 0{ }^{\circ} \mathrm{C}$ solution of $(\mathrm{S})-\mathrm{N}-(((1 \mathrm{R}, 3 \mathrm{~S}, 5 \mathrm{~S}, 6 \mathrm{r})-3-$ hydroxybicyclo[3.1.0]hexan-6-yl)methyl)-2-methylpropane-2-sulfinamide (1.3 g, $5.62 \mathrm{mmol})$ in DCM $(28.1 \mathrm{~mL})$ was added methanesulfonyl chloride $(0.657 \mathrm{~mL}, 8.43 \mathrm{mmol})$ and the resulting mixture was stirred and allowed to warm to RT over $3 \mathrm{~h}$. The reaction was dilluted with EtOAc $(200 \mathrm{~mL})$ and washed with $0.5 \mathrm{M} \mathrm{HCl}(100 \mathrm{~mL})$, water $(150 \mathrm{~mL}), 0.5 \mathrm{M} \mathrm{NaOH}(100 \mathrm{~mL})$, and 
brine $(100 \mathrm{~mL})$. Each aqueous layer was sequentially extracted with EtOAc $(100 \mathrm{~mL})$. The two organic layers were combined, dried over $\mathrm{MgSO} 4$, filtered, and concentrated to give the title compound $\mathbf{5 4 C}$ (step3) (1.43 g, $4.62 \mathrm{mmol}, 82 \%$ yield) as a semi-solid-wax. MS (ES ${ }^{+}$) $\mathrm{C}_{12} \mathrm{H}_{23} \mathrm{NO}_{4} \mathrm{~S} 2$ requires: 309 , found: $310[\mathrm{M}+\mathrm{H}]^{+}$.

Step 4: (S)-N-(((1R,3R,5S,6r)-3-(5,6-difluoro-1H-benzo[d]imidazol-1-yl)bicyclo[3.1.0]hexan-6yl)methyl)-2-methylpropane-2-sulfinamide 54C. To a solution of (1R,3S,5S,6r)-6-(((S)-1,1dimethylethylsulfinamido)methyl) bicyclo[3.1.0]hexan-3-yl methanesulfonate (1.42 g, 4.59 $\mathrm{mmol}) \mathbf{5 4 C}$ (step3) in THF $(15.30 \mathrm{~mL})$ was added cesium carbonate $(4.49 \mathrm{~g}, 13.77 \mathrm{mmol})$ and 5,6-difluoro-1H-benzo[d]imidazole $(0.849 \mathrm{~g}, 5.51 \mathrm{mmol})$ and the resulting mixture was stirred at $85^{\circ} \mathrm{C}$ for $16 \mathrm{~h}$. The reaction was cooled to RT, concentrated, diluted with water and extracted twice with EtOAc. The organics were washed with brine, combined, dried over MgSO4, concentrated and purified by flash chromatography ( 5 to $40 \%$ of $80: 20: 2 \mathrm{EA}: \mathrm{EtOH}: \mathrm{NH}_{4} \mathrm{OH}$ in hexanes) to give the title compound $\mathbf{5 4 C}(1.03 \mathrm{~g}, 2.80 \mathrm{mmol}, 61 \%$ yield $)$ as a white solid. MS $\left(\mathrm{ES}^{+}\right) \mathrm{C}_{18} \mathrm{H}_{23} \mathrm{~F}_{2} \mathrm{~N}_{3} \mathrm{OS}$ requires: 367 , found: $368[\mathrm{M}+\mathrm{H}]^{+}$.

Step 5: ((1R,3s,5S,6r)-3-(5,6-difluoro-1H-benzo[d]imidazol-1-yl)bicyclo[3.1.0]hexan-6$y l)$ methanamine dihydrochloride 54D. To a cooled $0{ }^{\circ} \mathrm{C}$ solution of $(\mathrm{S})-\mathrm{N}-(((1 \mathrm{R}, 3 \mathrm{R}, 5 \mathrm{~S}, 6 \mathrm{r})-3-$ (5,6-difluoro-1H-benzo[d]imidazol-1-yl)bicyclo[3.1.0]hexan-6-yl)methyl)-2-methylpropane-2sulfinamide 54C (1 g, $2.72 \mathrm{mmol})$ in $\mathrm{MeOH}(5.44 \mathrm{~mL})$ was added $\mathrm{HCl} 4 \mathrm{M}$ in Dioxane $(2.72 \mathrm{~mL}$, $10.89 \mathrm{mmol}$ ) and the resulting mixture was stirred at $0{ }^{\circ} \mathrm{C}$ for $5 \mathrm{~min}$ then at RT for $3 \mathrm{~h}$. The reaction was concentrated, dried by being azeotroped with $\mathrm{ACN}$ and DCM/Hexanes to give the title compound 54D $(0.9 \mathrm{~g}, 2.7 \mathrm{mmol}, 98 \%$ yield $)$ as a white solid. $\mathrm{MS}\left(\mathrm{ES}^{+}\right) \mathrm{C}_{14} \mathrm{H}_{15} \mathrm{~F}_{2} \mathrm{~N}_{3} \cdot 2 \mathrm{ClH}$ requires: 263 , found: $264[\mathrm{M}+\mathrm{H}]^{+}$.

Step 6: 4-chloro-N-(((1R,3s,5S,6r)-3-(5,6-difluoro-1H-benzo[d]imidazol-1yl)bicyclo[3.1.0]hexan-6-yl)methyl)benzamide 54. The title compound was synthesized from ((1R,3s,5S,6r)-3-(5,6-difluoro-1H-benzo[d]imidazol-1-yl)bicyclo[3.1.0]hexan-6-yl)methanamine dihydrochloride 54D in a reaction similar to the amide coupling procedure of compound 21 using DIEA, EDC, and HOBt to give the title compound 54 (26 mg, $0.065 \mathrm{mmol}, 43 \%$ yield). MS $\left(\mathrm{ES}^{+}\right) \mathrm{C}_{21} \mathrm{H}_{18} \mathrm{ClF}_{2} \mathrm{~N}_{3} \mathrm{O}$ requires: 401, found: $402[\mathrm{M}+\mathrm{H}]^{+} .{ }^{1} \mathrm{H}$ NMR (DMSO- $d_{6}$ ) $\delta: 8.68$ (t, J = 5.7 $\mathrm{Hz}, 1 \mathrm{H}), 8.43$ (s, 1H), 7.88 - 7.92 (m, 2H), 7.85-7.87 (m, 1H), 7.66-7.70 (m, 1H), 7.53 - 7.58 (m, 
2H), 4.56 - $4.66(\mathrm{~m}, 1 \mathrm{H}), 3.14-3.18(\mathrm{~m}, 2 \mathrm{H}), 2.30-2.34(\mathrm{~m}, 2 \mathrm{H}), 2.14-2.23(\mathrm{~m}, 2 \mathrm{H}), 1.43$ (br. s., $2 \mathrm{H}), 1.24-1.26(\mathrm{~m}, 1 \mathrm{H})$.

Compound 55: 1-(4-chlorophenyl)-N-((1R,5S)-3-(5,6-difluoro-1H-benzo[d]imidazol-1yl)bicyclo[3.1.0]hexan-6-yl)methyl)-2,2,2-trifluoroethan-1-amine

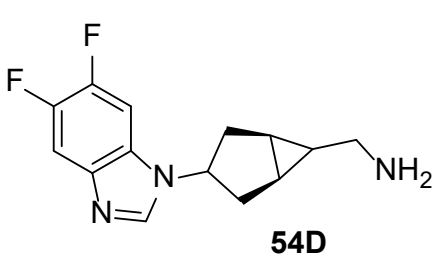

54D<smiles>FC(F)(F)C(O[Tl])c1ccc(Cl)cc1</smiles>

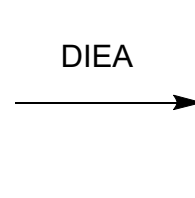

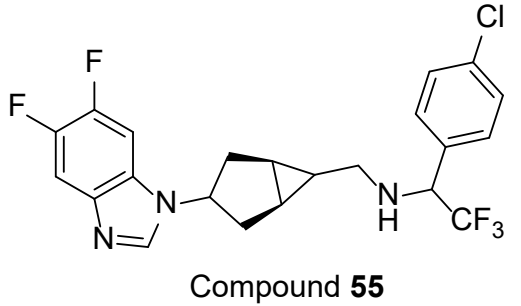

Compound 55

To a solution of ((1R,5S)-3-(5,6-difluoro-1H-benzo[d]imidazol-1-yl)bicyclo[3.1.0]hexan-6yl)methanamine dihydrochloride 54D (33 mg, $0.097 \mathrm{mmol})$ in DCM (970 $\mu 1)$ were added DIEA ( $85 \mu 1,0.49 \mathrm{mmol})$ and 1-(4-chlorophenyl)-2,2,2-trifluoroethyl trifluoromethanesulfonate (50 $\mathrm{mg}, 0.15 \mathrm{mmol}$ ) and the resulting mixture was stirred at $25^{\circ} \mathrm{C}$ for 5 days. The volatiles were removed under reduced pressure and the residue was purified via silica gel chromatography $(0$ $10 \% \mathrm{MeOH}$ in DCM with 2\% TEA to give the title compound $\mathbf{5 5}(10 \mathrm{mg}, 0.022 \mathrm{mmol}, 22 \%$ yield) as a yellow liquid. $\mathrm{MS}\left(\mathrm{ES}^{+}\right) \mathrm{C}_{22} \mathrm{H}_{19} \mathrm{ClF}_{5} \mathrm{~N}_{3}$ requires: 455, found: $456[\mathrm{M}+\mathrm{H}]^{+} .{ }^{1} \mathrm{H} \mathrm{NMR}$ (600 MHz, $\left.\mathrm{CD}_{3} \mathrm{OD}\right): \delta 8.26(\mathrm{~s}, 1 \mathrm{H}), 7.54-7.41(\mathrm{~m}, 6 \mathrm{H}), 4.55-4.50(\mathrm{~m}, 1 \mathrm{H}), 4.40-4.36(\mathrm{~m}$, $1 \mathrm{H}), 3.35-3.30(\mathrm{~m}, 2 \mathrm{H}), 2.47-2.21(\mathrm{~m}, 6 \mathrm{H}), 1.03(\mathrm{~s}, 1 \mathrm{H})$.

Compound 56: 4-chloro-N-((R)-1-((1R,3S,5S,6r)-3-(6-fluoro-1H-benzo[d]imidazol-1yl)bicyclo[3.1.0]hexan-6-yl)propyl)benzamide.

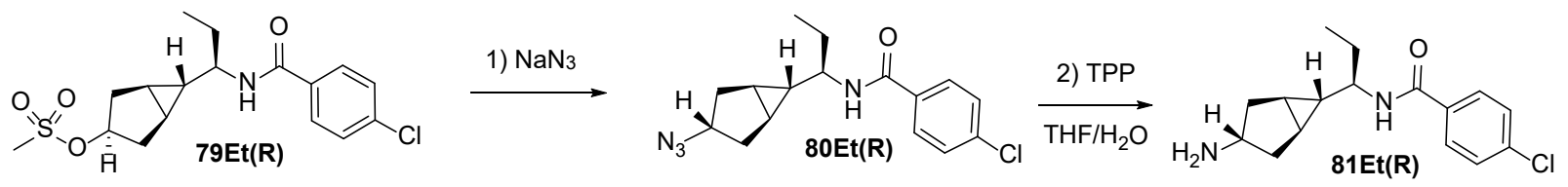

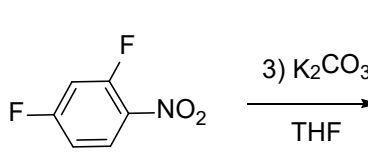

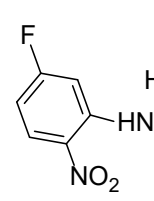

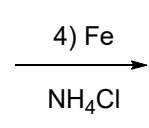

82Et(R)(5-F) $\mathrm{Cl}$

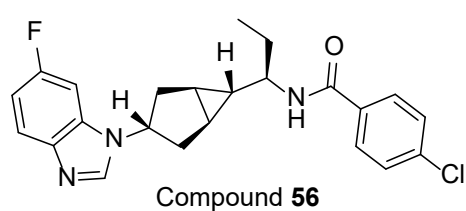

Compound 56

Compound $\quad \mathbf{8 0 E t}(\mathbf{R}): \quad N-((R)-1-((1 R, 3 S, 5 S, 6 r)-3$-azidobicyclo[3.1.0]hexan-6-yl)propyl)-4chlorobenzamide. 
Step 1. To a solution of (1R,3R,5S,6r)-6-((R)-1-(4-chlorobenzamido)propyl)bicyclo[3.1.0]hexan3-yl methanesulfonate 79Et(R) (500 mg, $01.34 \mathrm{mmol})$ in DMF (3.4 mL) was added sodium azide (350mg, 5.38mmol) and the resulting mixture was stirred at $85{ }^{\circ} \mathrm{C}$ for $1 \mathrm{~h}$. The reaction mixture was diluted with EtOAc $(50 \mathrm{~mL})$ and washed with $\mathrm{H}_{2} \mathrm{O}(50 \mathrm{~mL})$. The layers were separated, and the organic layer was washed with sat $\mathrm{NaCl}(50 \mathrm{~mL})$, dried over $\mathrm{Na}_{2} \mathrm{SO}_{4}$, filtered and concentrated under reduced pressure to give the title compound $80 \mathrm{Et}(\mathbf{R})(1.6 \mathrm{~g}, 5.0 \mathrm{mmol}, 93 \%$ yield) as an offwhite solid. The product was taken on without further purification. MS (ES $\left.{ }^{+}\right) \mathrm{C}_{16} \mathrm{H}_{19} \mathrm{ClN}_{4} \mathrm{O}$ requires: 318 , found: $319[\mathrm{M}+\mathrm{H}]^{+}$.

Compound $\quad \mathbf{8 1 E t ( R ) : ~} \quad N-((R)-1-((1 R, 3 S, 5 S, 6 r)-3-$ aminobicyclo[3.1.0]hexan-6-yl)propyl)-4chlorobenzamide

Step 2. To a solution of N-((R)-1-((1R,3S,5S,6r)-3-azidobicyclo[3.1.0]hexan-6-yl)propyl)-4chlorobenzamide $\mathbf{8 0 E t}(\mathbf{R})(1.2 \mathrm{~g}, 3.8 \mathrm{mmol})$ in THF $(10 \mathrm{~mL})$ were added triphenylphosphine $(3.0$ $\mathrm{g}, 11 \mathrm{mmol})$ and water $(1 \mathrm{~mL})$ and the resulting mixture was stirred at room temperature for $12 \mathrm{~h}$. The volatiles were removed under reduced pressure. The residue was purified by silica gel chromatography (50-100\%) Methanol/DCM/Ammonium hydroxide (20:80:1) in DCM to give the title compound $\mathbf{8 1 E t}(\mathbf{R})(880 \mathrm{mg}, 3.01 \mathrm{mmol}, 80 \%$ yield) as an off-white solid. MS (ES $)$ $\mathrm{C}_{16} \mathrm{H}_{21} \mathrm{ClN}_{2} \mathrm{O}$ requires: 292, found: $293[\mathrm{M}+\mathrm{H}]^{+}$.

Compound $\quad \mathbf{8 2 E t}(\mathbf{R})(\mathbf{5 - F}): \quad$ 4-chloro- $N-((R)-1-((1 R, 3 S, 5 S, 6 r)-3-((5-$ fluoro-2nitrophenyl)amino)bicyclo[3.1.0]hexan-6-yl)propyl)benzamide.

Step 3. To a solution of N-((R)-1-((1R,3S,5S,6r)-3-aminobicyclo[3.1.0]hexan-6-yl)propyl)-4chlorobenzamide 81Et(R) (500 mg, $1.7 \mathrm{mmol})$ in THF (10 mL) were added 2,4-difluoro-1nitrobenzene (300 $\mathrm{mg}, 1.9 \mathrm{mmol})$ and potassium carbonate $(472 \mathrm{mg}, 3.42 \mathrm{mmol})$ and the resulting mixture was stirred at room temperature for $12 \mathrm{~h}$. The reaction mixture was diluted with ethylacetate $(30 \mathrm{~mL})$ and washed with water $(10 \mathrm{~mL})$. The layers were separated, and the organic layer was washed with brine $(10 \mathrm{~mL})$, dried over $\mathrm{MgSO}_{4}$, filtered and concentrated under reduced pressure. The residue was purified by silica gel chromatography (10-80\% EtOAc in hexane to give the title compound $\mathbf{8 2 E t}(\mathbf{R})(\mathbf{5 - F})\left(610 \mathrm{mg}, 1.41 \mathrm{mmol}, 83 \%\right.$ yield) as a yellow solid. MS (ES $\left.{ }^{+}\right)$ $\mathrm{C}_{22} \mathrm{H}_{23} \mathrm{ClFN}_{3} \mathrm{O}_{3}$ requires:431, found: $432[\mathrm{M}+\mathrm{H}]^{+}$. 
Compound 56: 4-chloro-N-((R)-1-((1R,3S,5S,6r)-3-(6-fluoro-1H-benzo[d]imidazol-1yl)bicyclo[3.1.0]hexan-6-yl)propyl)benzamide.

Step 4. To a solution of 4-chloro-N-((R)-1-((1R,3S,5S,6r)-3-((5-fluoro-2nitrophenyl)amino)bicyclo[3.1.0]hexan-6-yl)propyl)benzamide 82Et(R)(5-F) (450mg, 1.04 $\mathrm{mmol})$ in isopropanol/formic acid $(1: 1)(10 \mathrm{~mL})$ were added iron powder $(582 \mathrm{mg}, 10.4 \mathrm{mmol})$ and ammonium chloride (560 mg, $10.4 \mathrm{mmol}$ ) and the resulting mixture was stirred at $80{ }^{\circ} \mathrm{C}$ for $12 \mathrm{~h}$. The reaction mixture was filtered, and the filtrate was concentrated under reduced pressure. The reaction mixture was diluted with EtOAc $(50 \mathrm{~mL})$ and washed with $5 \% \mathrm{NaOH}(15 \mathrm{~mL}, 2 \mathrm{x})$ and brine $(20 \mathrm{~mL})$, dried over $\mathrm{MgSO}_{4}$, filtered, and concentrated under reduced pressure. The residue was purified by silica gel chromatography (50-100\%) EtOAc/IPA (4:1) in hexane to give the title compound 56 (230 $\mathrm{mg}, 0.56 \mathrm{mmol}, 53 \%$ yield) as a white solid. HRMS (ES ${ }^{+}$) $\mathrm{C}_{23} \mathrm{H}_{23} \mathrm{ClFN}_{3} \mathrm{O}$ requires: $412.1586[\mathrm{M}+\mathrm{H}]^{+}$, found: 412.1581 . [M+H] $]^{+} .{ }^{1} \mathrm{H} \mathrm{NMR}(600 \mathrm{MHz}$, DMSO) $\delta 8.37$ (s, 1H), 8.32 (d, $J=8.4 \mathrm{~Hz}, 1 \mathrm{H}), 7.90(\mathrm{~d}, J=2.1 \mathrm{~Hz}, 2 \mathrm{H}), 7.65-7.58(\mathrm{~m}, 1 \mathrm{H})$, $7.54-7.48(\mathrm{~m}, 3 \mathrm{H}), 7.05-6.95(\mathrm{~m}, 1 \mathrm{H}), 4.68-4.52(\mathrm{~m}, 1 \mathrm{H}), 3.48-3.35(\mathrm{~m}, 1 \mathrm{H}), 2.45-2.10$ (m, 4H), $1.75-1.55(\mathrm{~m}, 2 \mathrm{H}), 1.52-1.35(\mathrm{~m}, 2 \mathrm{H}), 1.20-1.10(\mathrm{~m}, 1 \mathrm{H}), 0.94(\mathrm{t}, J=7.4 \mathrm{~Hz}, 3 \mathrm{H})$.

Compound 57: 4-chloro-N-[(1R)-1-[(1R,3s,5S,6R)-3-(6-fluoro-2-methyl-1H-1,3-benzodiazol1-yl)bicyclo[3.1.0]hexan-6-yl]propyl]benzamide.

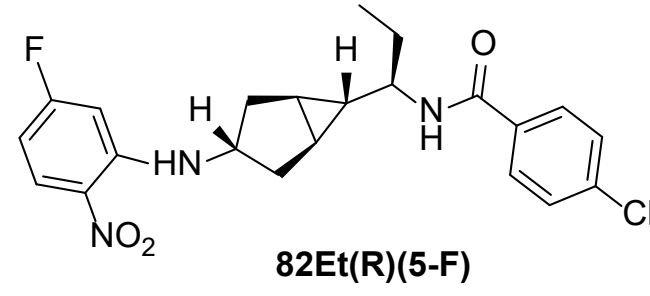

To

a

solution

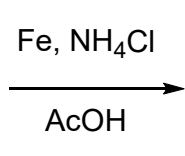

of

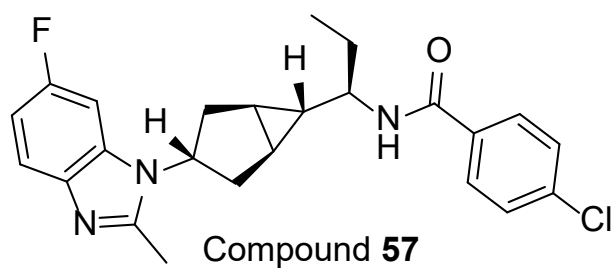

Compound $\mathbf{5 7}$

nitrophenyl)amino)bicyclo[3.1.0] hexan-6-yl)propyl)benzamide 82Et(R)(5-F) (100mg, $0.23 \mathrm{mmol})$ in IPA/acetic acid (1:1) (4ml) were added iron powder $(130 \mathrm{mg}, 2.3 \mathrm{mmol})$ and ammonium chloride (130 mg, $2.43 \mathrm{mmol}$ ) and the resulting mixture was stirred at $80^{\circ} \mathrm{C}$ for $12 \mathrm{~h}$. The reaction mixture was allowed to cool to room temperature. The reaction mixture was diluted with 2-propanol $(5 \mathrm{ml})$. The reaction mixture was filtered through Buchner funnel, and the filtrate was concentrated under reduced pressure. The reaction mixture was diluted with EtOAc $(20 \mathrm{~mL})$ 
and washed with $5 \% \mathrm{NaOH}(5 \mathrm{~mL})$. The layers were separated, and the organic layer was washed with $5 \% \mathrm{NaOH}(5 \mathrm{~mL})$ followed by brine $(1 \times 10 \mathrm{~mL})$, dried over $\mathrm{MgSO}_{4}$, filtered and concentrated under reduced pressure. The residue was purified by mass-triggered preparative HPLC (Mobile phase: $\mathrm{A}=0.1 \% \mathrm{TFA} / \mathrm{H}_{2} \mathrm{O}, \mathrm{B}=0.1 \% \mathrm{TFA} / \mathrm{MeCN}$; Gradient: $\mathrm{B}=20$ - 60\%; 12 min; Column: $\mathrm{C} 18)$ to give the title compound 57 (23 mg, $0.054 \mathrm{mmol}, 23 \%$ yield) as a white powder. $\mathrm{MS}_{\left(\mathrm{ES}^{+}\right)}$ $\mathrm{C}_{24} \mathrm{H}_{25} \mathrm{ClFN}_{3} \mathrm{O}$ requires 425, found 426. ${ }^{1} \mathrm{H} \mathrm{NMR}(600 \mathrm{MHz}, \mathrm{DMSO}-\mathrm{d} 6) \delta 8.32$ (d, J = 8.4 Hz, 1H), $7.90(\mathrm{~d}, \mathrm{~J}=8.4 \mathrm{~Hz}, 2 \mathrm{H}), 7.55(\mathrm{~d}, \mathrm{~J}=9.1 \mathrm{~Hz}, 2 \mathrm{H}), 7.48(\mathrm{dd}, \mathrm{J}=9.3,2.2 \mathrm{~Hz}, 1 \mathrm{H}), 7.25-7.20$ (m, 1H), 6.98- $6.90(\mathrm{~m}, 1 \mathrm{H}), 4.65-4.56(\mathrm{~m}, 1 \mathrm{H}), 3.46-3.37(\mathrm{~m}, 1 \mathrm{H}), 2.51(\mathrm{~s}, 3 \mathrm{H}), 2.39-2.36$ $(\mathrm{m}, 2 \mathrm{H}), 2.20-2.16(\mathrm{~m}, 1 \mathrm{H}), 2.11-2.07(\mathrm{~m}, 1 \mathrm{H}), 1.76-1.62(\mathrm{~m}, 2 \mathrm{H}), 1.53-1.47(\mathrm{~m}, 2 \mathrm{H}), 1.10$ $-1.06(\mathrm{~m}, 1 \mathrm{H}), 0.92(\mathrm{t}, J=7.4 \mathrm{~Hz}, 3 \mathrm{H})$.

Compound 58: 4-chloro-N-((R)-1-((1R,3S,5S,6r)-3-(6-chloro-1H-benzo[d]imidazol-1yl)bicyclo[3.1.0]hexan-6-yl)propyl)benzamide.
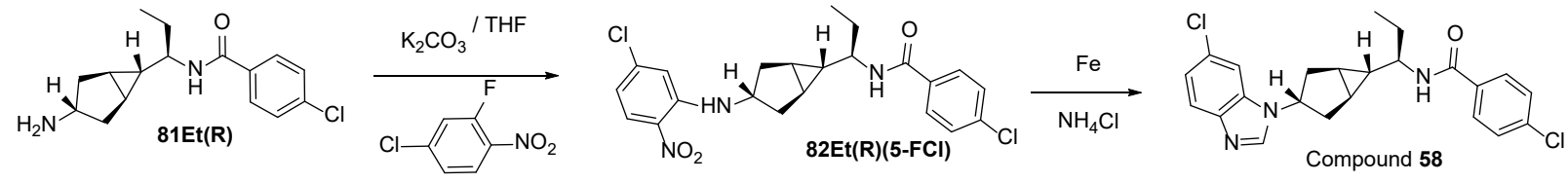

The title compound was synthesized similar to compound 56 from $\mathbf{8 1 E t}(\mathbf{R})$ (420 mg, 0.34 mmol), 4-chloro-2-fluoro-1-nitrobenzene (264 mg, $1.51 \mathrm{mmol}$ ) and $\mathrm{K}_{2} \mathrm{CO}_{3}(396 \mathrm{mg}, 2.87 \mathrm{mmol})$ in THF (14 mL) to give intermediate compound 82Et(R)(5-Cl) (434 mg, $0.97 \mathrm{mmol}, 67 \%)$ that was converted to the benzimidazole with iron $(540 \mathrm{mg}, 9.7 \mathrm{mmol})$ and $\mathrm{NH}_{4} \mathrm{Cl}(520 \mathrm{mg}, 9.7$ mmol) in IPA:FA (3:3 mL) to give $58(313 \mathrm{mg}, 0.73 \mathrm{mmol}, 75 \%$ yield $)$ as an off-white solid. MS (ES $\left.{ }^{+}\right) \mathrm{C}_{23} \mathrm{H}_{23} \mathrm{Cl}_{2} \mathrm{FN}_{3} \mathrm{O}$ requires: 427, found: $428[\mathrm{M}+\mathrm{H}]^{+} .{ }^{1} \mathrm{H}$ NMR (600 MHz, DMSO-d 6 ) $\delta$ $8.41(\mathrm{~s}, 1 \mathrm{H}), 8.33(\mathrm{~d}, J=8.5 \mathrm{~Hz}, 1 \mathrm{H}), 7.91$ (d, $J=8.3 \mathrm{~Hz}, 2 \mathrm{H}), 7.80$ (d, $J=2.0 \mathrm{~Hz}, 1 \mathrm{H}), 7.64$ (d, $J=8.6 \mathrm{~Hz}, 1 \mathrm{H}), 7.55(\mathrm{~d}, J=8.3 \mathrm{~Hz}, 2 \mathrm{H}), 7.21(\mathrm{dd}, J=8.4,2.0 \mathrm{~Hz}, 1 \mathrm{H}), 4.71-4.61(\mathrm{~m}, 1 \mathrm{H})$, $3.49-3.39(\mathrm{~m}, 1 \mathrm{H}), 2.40-2.15(\mathrm{~m}, 4 \mathrm{H}), 1.74-1.60(\mathrm{~m}, 2 \mathrm{H}), 1.53-1.37(\mathrm{~m}, 2 \mathrm{H}), 1.21-1.15$ $(\mathrm{m}, 1 \mathrm{H}), 0.94(\mathrm{t}, J=7.4 \mathrm{~Hz}, 3 \mathrm{H})$.

Compound 59: 4-chloro-N-((R)-1-((1R,3S,5S,6r)-3-(6-cyano-1H-benzo[d]imidazol-1yl)bicyclo[3.1.0]hexan-6-yl)propyl)benzamide 

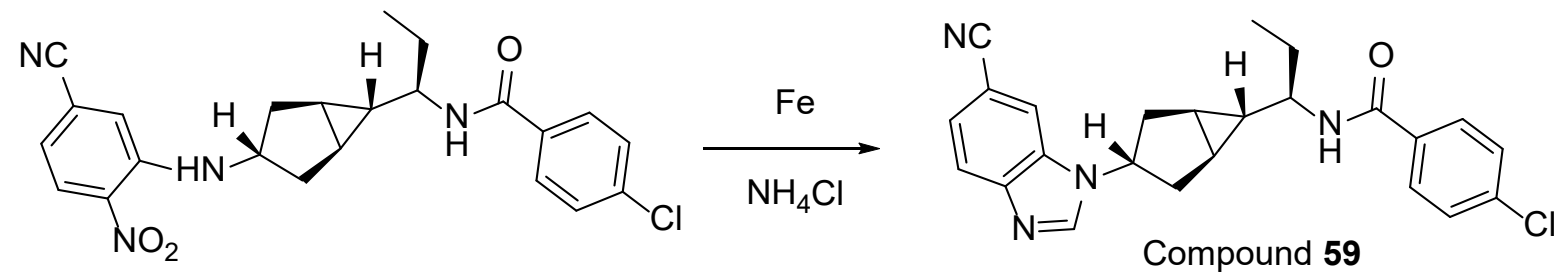

The title compound was synthesized similar to compound 41. HRMS $\left(\mathrm{ES}^{+}\right) \mathrm{C}_{24} \mathrm{H}_{23} \mathrm{ClN}_{4} \mathrm{O}$ requires $419.1633[\mathrm{M}+\mathrm{H}]^{+}$, found $419.1626[\mathrm{M}+\mathrm{H}]^{+} .{ }^{1} \mathrm{H}$ NMR (500 MHz, DMSO) $\delta 8.64(\mathrm{~s}, 1 \mathrm{H}), 8.34-$ $8.32(\mathrm{~m}, 2 \mathrm{H}), 7.90(\mathrm{~d}, \mathrm{~J}=8.6 \mathrm{~Hz}, 2 \mathrm{H}), 7.81-7.79(\mathrm{~m}, 1 \mathrm{H}), 7.58-7.54(\mathrm{~m}, 3 \mathrm{H}), 4.71-4.65(\mathrm{~m}$, 1H), 3.43 (dd, $J=8.4,6.3 \mathrm{~Hz}, 1 \mathrm{H}$ ), 2.39 (dd, $J=12.4,7.7 \mathrm{~Hz}, 1 \mathrm{H}), 2.31-2.23$ (m, 2H), $2.24-$ $2.14(\mathrm{~m}, 1 \mathrm{H}), 1.76-1.62(\mathrm{~m}, 2 \mathrm{H}), 1.57-1.39(\mathrm{~m}, 2 \mathrm{H}), 1.23-1.13(\mathrm{~m}, 1 \mathrm{H}), 0.94(\mathrm{t}, J=7.4 \mathrm{~Hz}$, $3 \mathrm{H})$.

Compound $\quad 60: \quad \quad$ N-((R)-1-((1R,3S,5S,6r)-3-(6-chloro-1H-benzo[d]imidazol-1yl)bicyclo[3.1.0]hexan-6-yl)propyl)-4-cyanobenzamide<smiles>CCC(NC(=O)c1ccc(Cl)cc1)C(CC)NC(=O)c1ccc(C#N)cc1</smiles>

The title compound was synthesized similar to compound 58 starting from N-((R)-1((1R,3S,5S,6r)-3-aminobicyclo[3.1.0] hexan-6-yl)propyl)-4-cyanobenzamide which was synthesized similar to $81 \mathrm{Et}(\mathbf{R})$. MS $\left(\mathrm{ES}^{+}\right) \mathrm{C}_{24} \mathrm{H}_{23} \mathrm{ClN}_{4} \mathrm{O}$ requires 418 , found $419 .{ }^{1} \mathrm{H}$ NMR (600 MHz, DMSO- $\left.d_{6}\right) \delta 8.41(\mathrm{~s}, 1 \mathrm{H}), 8.51(\mathrm{~d}, J=8.4 \mathrm{~Hz}, 1 \mathrm{H}), 8.04-8.01(\mathrm{~m}, 2 \mathrm{H}), 7.99-7.95(\mathrm{~m}$, 2H), $7.80(\mathrm{~d}, J=2.1 \mathrm{~Hz}, 1 \mathrm{H}), 7.63(\mathrm{~d}, J=8.5 \mathrm{~Hz}, 1 \mathrm{H}), 7.20$ (dd, $J=8.5,2.0 \mathrm{~Hz}, 1 \mathrm{H}), 4.71-4.60$ (m, $1 \mathrm{H}), 3.46-3.38(\mathrm{~m}, 1 \mathrm{H}), 2.45-2.11(\mathrm{~m}, 4 \mathrm{H}), 1.68(\mathrm{dp}, J=14.2,6.9 \mathrm{~Hz}, 2 \mathrm{H}), 1.52-1.38(\mathrm{~m}$, $2 \mathrm{H}), 1.21-1.15(\mathrm{~m}, 1 \mathrm{H}), 0.94(\mathrm{t}, J=7.4 \mathrm{~Hz}, 3 \mathrm{H})$.

Compound 61: 4-cyano-N-((R)-1-((1R,3S,5S,6r)-3-(6-cyano-1H-benzo[d]imidazol-1yl)bicyclo[3.1.0]hexan-6-yl)propyl)benzamide 

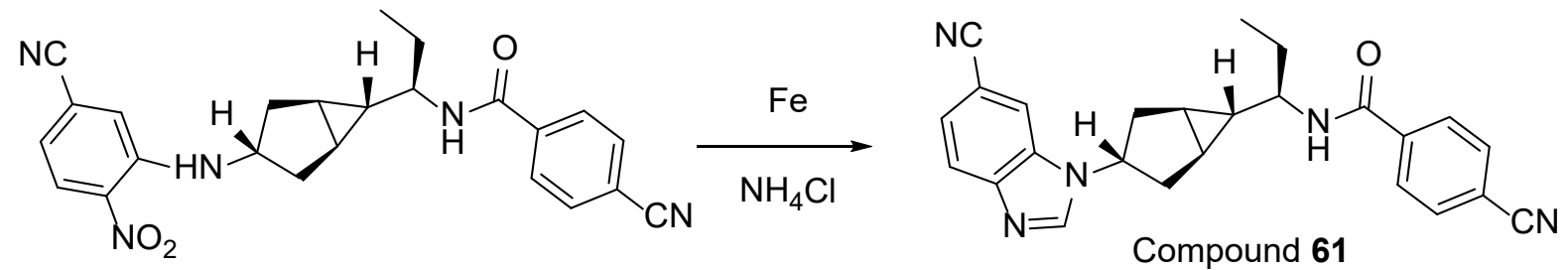

The title compound was synthesized similar to compound 41 starting from N-((R)-1((1R,3S,5S,6r)-3-aminobicyclo[3.1.0]hexan-6-yl)propyl)-4-cyanobenzamide which was synthesized similar to 81Et(R). MS (ES $\left.{ }^{+}\right) \mathrm{C}_{25} \mathrm{H}_{23} \mathrm{~N}_{5} \mathrm{O}$ requires 409, found 410. ${ }^{1} \mathrm{H}$ NMR (500 MHz, DMSO) $\delta 8.65(\mathrm{~s}, 1 \mathrm{H}), 8.53(\mathrm{~d}, J=8.4 \mathrm{~Hz}, 1 \mathrm{H}), 8.36-8.32(\mathrm{~m}, 1 \mathrm{H}), 8.03(\mathrm{~d}, J=8.6 \mathrm{~Hz}$, 2H), 7.97 (d, $J=8.6 \mathrm{~Hz}, 2 \mathrm{H}), 7.80$ (d, $J=8.4 \mathrm{~Hz}, 1 \mathrm{H}), 7.58$ (dd, $J=8.3,1.5 \mathrm{~Hz}, 1 \mathrm{H}), 4.79-4.65$ (m, 1H), 3.43 (dd, $J=8.4,6.3 \mathrm{~Hz}, 1 \mathrm{H}), 2.39$ (dd, $J=12.4,7.7 \mathrm{~Hz}, 1 \mathrm{H}), 2.31-2.23$ (m, 2H), 2.24 $-2.14(\mathrm{~m}, 1 \mathrm{H}), 1.76-1.62(\mathrm{~m}, 2 \mathrm{H}), 1.57-1.39(\mathrm{~m}, 2 \mathrm{H}), 1.23-1.13(\mathrm{~m}, 1 \mathrm{H}), 0.94(\mathrm{t}, J=7.4 \mathrm{~Hz}$, $3 \mathrm{H})$.

Compound 63: 4-chloro-N-((R)-1-((1R,3S,5S,6r)-3-(5,6-difluoro-1H-benzo[d]imidazol-1yl)bicyclo[3.1.0]hexan-6-yl)propyl)-3-fluorobenzamide
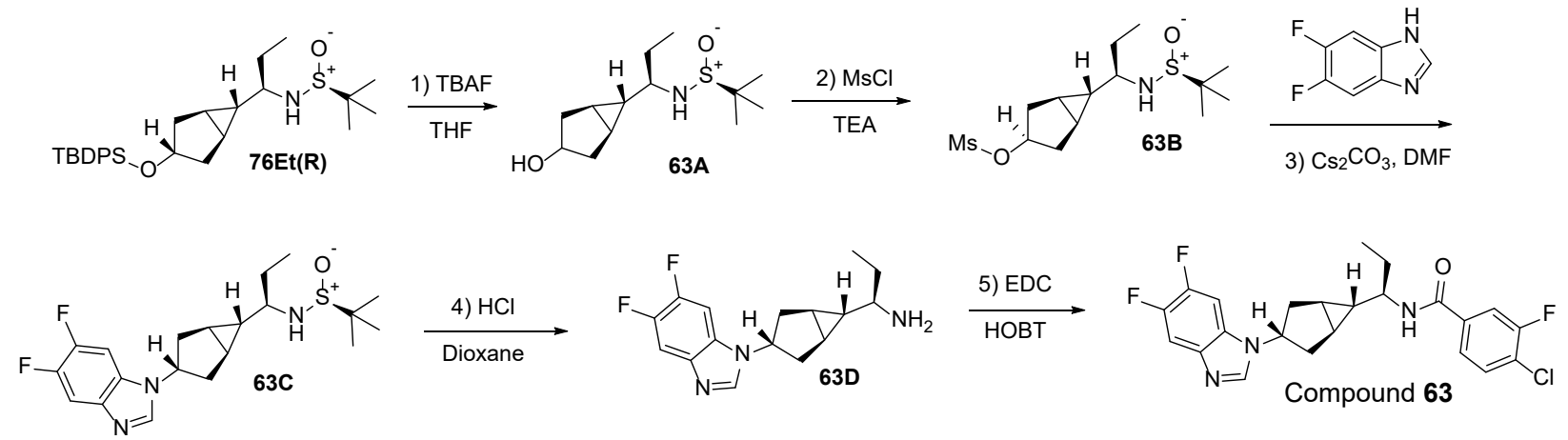

Step 1: (S)-N-((1R)-1-((1R,5S,6r)-3-hydroxybicyclo[3.1.0]hexan-6-yl)propyl)-2-methylpropane2-sulfinamide 63A.

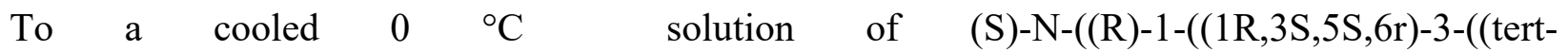
butyldiphenylsilyl)oxy)bicyclo[3.1.0]hexan-6-yl)propyl)-2-methylpropane-2-sulfinamide 76Et(R) (29 g, $58.3 \mathrm{mmol})$ in THF $(58.3 \mathrm{~mL})$ were added TBAF $(87 \mathrm{~mL}, 87 \mathrm{mmol})$. The resulting mixture was stirred at $25^{\circ} \mathrm{C}$ for $12 \mathrm{~h}$. The volatiles were removed under reduced pressure. The reaction mixture was diluted with EtOAc $(100 \mathrm{~mL})$ and washed with $\mathrm{H} 2 \mathrm{O}(3 \mathrm{x} 100 \mathrm{~mL})$. The 
layers were separated, and the organic layer was washed with saturated $\mathrm{NaCl}(100 \mathrm{~mL})$, dried over $\mathrm{Na}_{2} \mathrm{SO}_{4}$, filtered and concentrated under reduced pressure. The residue was purified by flash chromatography $(0-5 \% \mathrm{MeOH}$ in DCM) to give the title compound $\mathbf{6 3 A}(13.2 \mathrm{~g}, 50.9 \mathrm{mmol}, 87 \%$ yield) as a pale yellow liquid. $\mathrm{MS}\left(\mathrm{ES}^{+}\right) \mathrm{C}_{13} \mathrm{H}_{25} \mathrm{NO}_{2} \mathrm{~S}$ requires: 259 , found: $260[\mathrm{M}+\mathrm{H}]^{+}$.

Step 2: (1R,3R,5S,6r)-6-((R)-1-(((S)-tert-butylsulfinyl)amino)propyl)bicyclo[3.1.0] hexan-3-yl methanesulfonate $\mathbf{6 3 B}$.

The title compound was sythesized similar to compound 79Et(R) and used as is in the next step.

Step 3: $\quad(S)-N-((R)-1-((1 R, 3 S, 5 S, 6 r)-3-(5,6-d i f l u o r o-1 H-b e n z o[d]$ imidazol-1yl)bicyclo[3.1.0]hexan-6-yl)propyl)-2-methylpropane-2-sulfinamide 63C.

To a solution of $\quad(1 \mathrm{R}, 3 \mathrm{R}, 5 \mathrm{~S}, 6 \mathrm{r})-6-((\mathrm{R})-1-((\mathrm{S})$-tertbutylsulfinyl)amino)propyl)bicyclo[3.1.0] hexan-3-yl methanesulfonate 63B (1.0 g, $2.9 \mathrm{mmol})$ in DMF (29.6 mL) was added 5,6-difluoro-1H-benzo[d]imidazole (0.55 g, $3.6 \mathrm{mmol})$ and cesium carbonate $(2.4 \mathrm{~g}, 7.4 \mathrm{mmol})$ and the resulting mixture was stirred at $85{ }^{\circ} \mathrm{C}$ for $2 \mathrm{~h}$. The reaction mixture was allowed to cool to room temperature, $\mathrm{H}_{2} \mathrm{O}(10 \mathrm{~mL})$ was added, and the layers were separated. The aqueous phase was extracted with DCM $(3 \times 20 \mathrm{~mL})$, the combined organic layers were washed with saturated $\mathrm{NaCl}$, dried over $\mathrm{Na}_{2} \mathrm{SO}_{4}$, filtered and concentrated under reduced pressure. The residue was purified via silica gel chromatography $(0-10 \% \mathrm{MeOH}$ in DCM with $2 \%$ TEA to give the title compound $63 \mathrm{C}$ (735 $\mathrm{mg}, 1.86 \mathrm{mmol}, 62 \%$ yield) as a pale yellow foam solid. MS (ES ${ }^{+} \mathrm{C}_{20} \mathrm{H}_{27} \mathrm{~F}_{2} \mathrm{~N}_{3} \mathrm{OS}$ requires: 395, found: $396[\mathrm{M}+\mathrm{H}]^{+}$.

Step 4: (R)-1-((1R,3S,5S,6r)-3-(5,6-difluoro-1H-benzo[d]imidazol-1-yl)bicyclo[3.1.0]hexan-6yl)propan-1-amine 63D.

To a cooled $0{ }^{\circ} \mathrm{C}$ solution of $(\mathrm{S})-\mathrm{N}-((\mathrm{R})-1-((1 \mathrm{R}, 3 \mathrm{~S}, 5 \mathrm{~S}, 6 \mathrm{r})-3-(5,6-$ difluoro-1H-benzo[d]imidazol1-yl)bicyclo[3.1.0]hexan-6-yl)propyl)-2-methylpropane-2-sulfinamide 63C (735 mg, $1.86 \mathrm{mmol}$ ) in Dioxane $(3.7 \mathrm{~mL})$ was added $\mathrm{HCl}$ (4M dioxane, $2.3 \mathrm{~mL}, 9.2 \mathrm{mmol})$. The resulting mixture was stirred at $25{ }^{\circ} \mathrm{C}$ for $12 \mathrm{~h}$. The reaction mixture was cooled to $0{ }^{\circ} \mathrm{C}$ and TEA $(1321 \mu 1,9.48 \mathrm{mmol})$ was added slowly. The reaction mixture was allowed to warm to room temperature and upon completion was concentrated to give the title compound 63D and used without further purification as a yellow solid. $\mathrm{MS}\left(\mathrm{ES}^{+}\right) \mathrm{C}_{16} \mathrm{H}_{19} \mathrm{~F}_{2} \mathrm{~N}_{3}$ requires: 291, found: $292[\mathrm{M}+\mathrm{H}]^{+}$. 
Step 5: 4-chloro- $N-((R)-1-((1 R, 3 S, 5 S, 6 r)-3-(5,6$-difluoro-1H-benzo[d]imidazol-1yl)bicyclo[3.1.0]hexan-6-yl)propyl)-3-fluorobenzamide 63.

To a solution of amine 63D (163 mg, $0.558 \mathrm{mmol})$ in DCM ( $5 \mathrm{~mL})$ was added acid $(98 \mathrm{mg}, 0.56$ $\mathrm{mmol})$, HOBT (123 mg, $0.725 \mathrm{mmol})$, and DIEA ( $292 \mu \mathrm{l}, 1.67 \mathrm{mmol})$ and the resulting solution was stirred at $25^{\circ} \mathrm{C}$ for $5 \mathrm{~min}$. EDC $(117 \mathrm{mg}, 0.614 \mathrm{mmol})$ was added to the reaction mixture and the resulting solution was allowed to stir at $25{ }^{\circ} \mathrm{C}$ for $72 \mathrm{~h}$. The mixture was concentrated and purified by flash chromatography $(0-10 \% \mathrm{MeOH}$ in DCM) to give title compound 63 (195 $\mathrm{mg}, 0.436 \mathrm{mmol}, 78 \%$ yield). $\mathrm{MS}\left(\mathrm{ES}^{+}\right) \mathrm{C}_{23} \mathrm{H}_{21} \mathrm{ClF}_{3} \mathrm{~N}_{3} \mathrm{O}$ requires: 291, found: $292[\mathrm{M}+\mathrm{H}]^{+}{ }^{1} \mathrm{H}$ NMR (600 MHz, Chloroform- $d$ ) $\delta 7.92$ (s, 1H), $7.62-7.51$ (m, 2H), $7.52-7.46(\mathrm{~m}, 2 \mathrm{H}), 7.16$ (dd, $J=9.9,6.9 \mathrm{~Hz}, 1 \mathrm{H}), 5.93$ (d, $J=8.6 \mathrm{~Hz}, 1 \mathrm{H}), 4.35$ (p, $J=8.8 \mathrm{~Hz}, 1 \mathrm{H}), 3.56-3.45$ (m, 1H), $2.51-2.34(\mathrm{~m}, 2 \mathrm{H}), 2.35-2.24(\mathrm{~m}, 2 \mathrm{H}), 1.83-1.65(\mathrm{~m}, 3 \mathrm{H}), 1.62-1.57(\mathrm{~m}, 1 \mathrm{H}), 1.03(\mathrm{t}, J=$ $7.4 \mathrm{~Hz}, 3 \mathrm{H}), 0.97-0.88(\mathrm{~m}, 1 \mathrm{H})$.

Compound 64: 4-cyano-N-((R)-1-((1R,3S,5S,6r)-3-(5,6-difluoro-1H-benzo[d]imidazol-1yl)bicyclo[3.1.0]hexan-6-yl)propyl)benzamide.
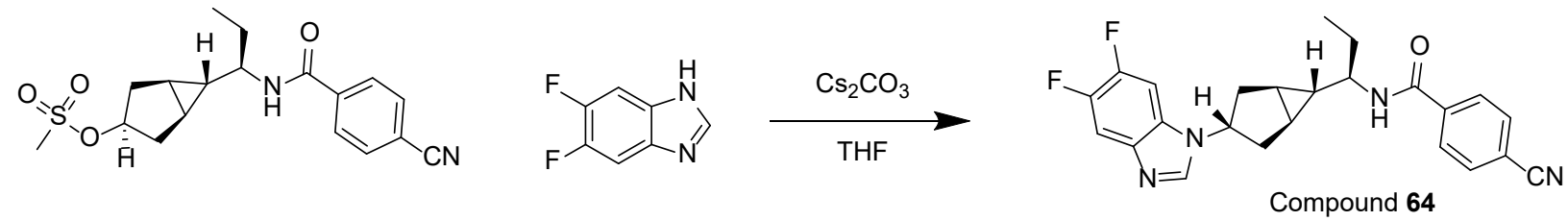

To a solution of (1R,3R,5S,6r)-6-((R)-1-(4-cyanobenzamido)propyl)bicyclo[3.1.0]hexan-3-yl methanesulfonate (324 mg, $0.894 \mathrm{mmol}$; synthesized similarly to compound $\mathbf{8 1 E t}(\mathbf{R})$ in THF (3 $\mathrm{mL}$ ) were added cesium carbonate $(874 \mathrm{mg}, 2.68 \mathrm{mmol})$ and 5,6-difluoro-1H-benzo[d]imidazole (152 $\mathrm{mg}, 0.983 \mathrm{mmol}$ ) and the resulting mixture was stirred at $65^{\circ} \mathrm{C}$ overnight. The reaction was cooled to room temperature, diluted with DCM, celite was added, and filtered over celite. The filtrate was concentrated and purified by flash chromatography ( 0 to $70 \%, 80: 20: 1: 1$ EA:IPA:MeOH:NH${ }_{4} \mathrm{OH}$ in hexanes) to give the title compound 64 (202 mg, $0.48 \mathrm{mmol}, 53 \%$ yield) as an off white solid. HRMS (ES $\left.{ }^{+}\right) \mathrm{C}_{24} \mathrm{H}_{22} \mathrm{~F}_{2} \mathrm{~N}_{4} \mathrm{O}$ requires: $421.1834[\mathrm{M}+\mathrm{H}]^{+}$, found: $421.1828[\mathrm{M}+\mathrm{H}]^{+} .{ }^{1} \mathrm{H}$ NMR $(600 \mathrm{MHz}, \mathrm{DMSO}) \delta 8.52(\mathrm{~d}, J=8.4 \mathrm{~Hz}, 1 \mathrm{H}), 8.43(\mathrm{~s}, 1 \mathrm{H}), 8.02(\mathrm{~d}$, $J=7.9 \mathrm{~Hz}, 2 \mathrm{H}), 7.97(\mathrm{~d}, J=7.9 \mathrm{~Hz}, 2 \mathrm{H}), 7.84(\mathrm{dd}, J=10.9,7.4 \mathrm{~Hz}, 1 \mathrm{H}), 7.69(\mathrm{dd}, J=11.3,7.5$ 
$\mathrm{Hz}, 1 \mathrm{H}), 4.66-4.55(\mathrm{~m}, 1 \mathrm{H}), 3.41(\mathrm{q}, J=7.9 \mathrm{~Hz}, 1 \mathrm{H}), 2.41-2.10(\mathrm{~m}, 4 \mathrm{H}), 1.74-1.59(\mathrm{~m}, 2 \mathrm{H})$, $1.53-1.38(\mathrm{~m}, 2 \mathrm{H}), 1.18-1.12(\mathrm{~m}, 1 \mathrm{H}), 0.93(\mathrm{t}, J=7.6 \mathrm{~Hz}, 3 \mathrm{H})$.

Compound 65: 4-chloro-N-((R)-1-((1R,3S,5S,6r)-3-(5,6-difluoro-1H-benzo[d]imidazol-1yl)bicyclo[3.1.0]hexan-6-yl)ethyl)benzamide.
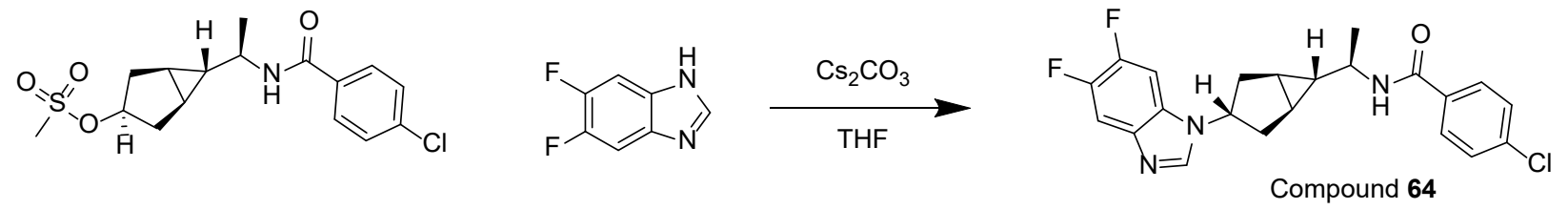

To a solution of (1R,3R,5S,6r)-6-((R)-1-(4-chlorobenzamido)ethyl)bicyclo[3.1.0]hexan-3-yl methanesulfonate $(4.0 \mathrm{~g}, 11 \mathrm{mmol}$; synthesized similarly to compound 81Et(R) in THF (37 $\mathrm{mL})$ were added cesium carbonate $(11 \mathrm{~g}, 12 \mathrm{mmol})$ and 5,6-difluoro-1H-benzo[d]imidazole (1.9 g, $12 \mathrm{mmol}$ ) and the resulting mixture was stirred at $65^{\circ} \mathrm{C}$ overnight. To the reaction was add more cesium carbonate $(0.9 \mathrm{~g})$ and 5,6-difluoro-1H-benzo[d]imidazole (190 mg) and heated again at $65^{\circ} \mathrm{C}$ overnight. The reaction was diluted with DCM, filter through celite, concentrate, support on silica gel and purified by flash chromatography (5-60\% of 80:20:1

EtOAc:EtOH: $\mathrm{NH}_{4} \mathrm{OH}$ in hexanes). The isolated desired product (2.6 g) was crystallized from EtOH to give the title compound $\mathbf{6 5}(1.3 \mathrm{~g}, 3.1 \mathrm{mmol}, 28 \%$ yield) as an off-white (grey) solid. MS (ES $\left.{ }^{+}\right) \mathrm{C}_{22} \mathrm{H}_{20} \mathrm{ClF}_{2} \mathrm{~N}_{3} \mathrm{O}$ requires:415, found: $416[\mathrm{M}+\mathrm{H}]^{+} .{ }^{1} \mathrm{H}$ NMR (600 MHz, DMSO-d $) \delta$ $8.46-8.41(\mathrm{~m}, 2 \mathrm{H}), 7.90(\mathrm{~d}, J=8.3 \mathrm{~Hz}, 2 \mathrm{H}), 7.84(\mathrm{dd}, J=10.9,7.3 \mathrm{~Hz}, 1 \mathrm{H}), 7.69$ (dd, $J=11.2$, $7.5 \mathrm{~Hz}, 1 \mathrm{H}), 7.55(\mathrm{~d}, J=8.3 \mathrm{~Hz}, 2 \mathrm{H}), 4.65-4.55(\mathrm{~m}, 1 \mathrm{H}), 3.64-3.55(\mathrm{~m}, 1 \mathrm{H}), 2.32$ (ddd, $J=$ 40.2, 12.4, 7.6 Hz, 2H), $2.25-2.14(\mathrm{~m}, 2 \mathrm{H}), 1.55-1.37(\mathrm{~m}, 2 \mathrm{H}), 1.25$ (d, J=6.6 Hz, 3H), 1.19 $(\mathrm{dt}, J=7.3,3.3 \mathrm{~Hz}, 1 \mathrm{H})$.

Compound 66: 4-chloro-N-(2-((1R,3s,5S,6r)-3-(5,6-difluoro-1H-benzo[d]imidazol-1yl)bicyclo[3.1.0]hexan-6-yl)propan-2-yl)benzamide. 

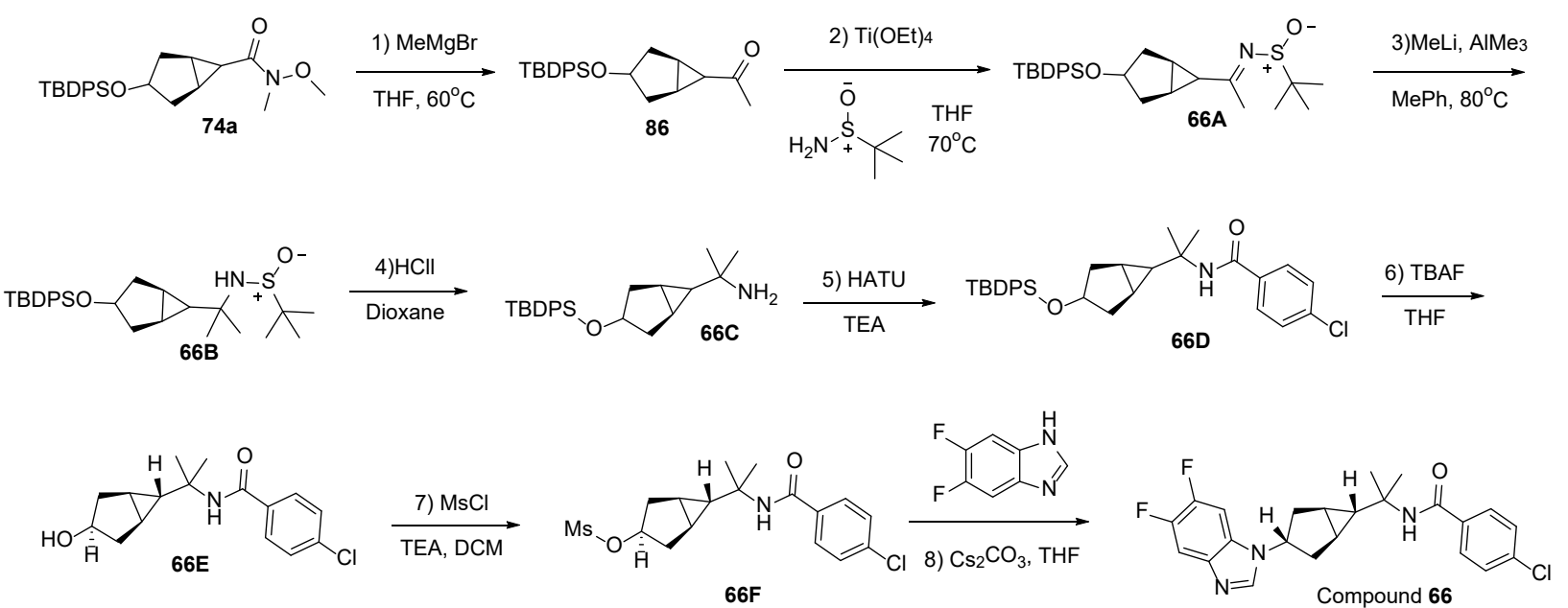

Step 1:1-((1R,5S)-3-((tert-butyldiphenylsilyl)oxy)bicyclo[3.1.0]hexan-6-yl)ethanone 86.

To a cooled $0{ }^{\circ} \mathrm{C}$ solution of (1R,5S)-3-((tert-butyldiphenylsilyl)oxy)-N-methoxy-Nmethylbicyclo[3.1.0]hexane-6-carboxamide 74a (step a for 74 from 73) (2.74 g, $6.47 \mathrm{mmol}$ ) in THF (11 mL) was added methylmagnesium bromide (1M in THF) (12.94 mL, $12.94 \mathrm{mmol})$ and the resulting mixture was stirred at $0{ }^{\circ} \mathrm{C}$ for $10 \mathrm{~min}$ and then at $60{ }^{\circ} \mathrm{C}$ overnight. The reaction was allowed to cool to room temperature, diluted with EtOAc $(100 \mathrm{~mL})$, and washed with $1 \mathrm{M}$ $\mathrm{HCl}(100 \mathrm{~mL})$ and saturated $\mathrm{NaCl}(100 \mathrm{~mL})$, dried over $\mathrm{Na}_{2} \mathrm{SO}_{4}$, filtered, and concentrated to give the title compound 86 (1.88 g, 4.97 mmol, 77\% yield) as a clear oil. Product was taken on without further purification. $\mathrm{MS}\left(\mathrm{ES}^{+}\right) \mathrm{C}_{24} \mathrm{H}_{30} \mathrm{O}_{2}$ Si requires: 378 , found: $401[\mathrm{M}+\mathrm{Na}]^{+}$.

Step 2: N-((E)-1-((1R,5S)-3-((tert-butyldiphenylsilyl)oxy)bicyclo[3.1.0]hexan-6-yl)ethylidene)2-methylpropane-2-sulfinamide 66.

To a solution of 1-((1R,5S)-3-((tert-butyldiphenylsilyl)oxy)bicyclo[3.1.0]hexan-6-yl)ethanone (1.88 g, $4.97 \mathrm{mmol}) 86$ in THF (4 mL) were added 2-methylpropane-2-sulfinamide $(0.722 \mathrm{~g}$, $5.96 \mathrm{mmol})$ and tetraethoxytitanium $(2.67 \mathrm{~g}, 9.93 \mathrm{mmol})$ and the resulting mixture was stirred at $70{ }^{\circ} \mathrm{C}$ for $44 \mathrm{~h}$. The residue was adsorbed onto silica gel and purified via flash chromatography (0-100\% EtOAc in hexanes) to give the title compound 66A (1.85 g, $3.84 \mathrm{mmol}, 77 \%$ yield $)$ as a clear oil. $\mathrm{MS}\left(\mathrm{ES}^{+}\right) \mathrm{C}_{28} \mathrm{H}_{39} \mathrm{NO}_{2} \mathrm{SSi}$ requires: 481, found: $482[\mathrm{M}+\mathrm{H}]^{+}$.

Step 3: N-(2-((1R,5S)-3-((tert-butyldiphenylsilyl)oxy)bicyclo[3.1.0]hexan-6-yl)propan-2-yl)-2methylpropane-2-sulfinamide 66B. 
To a stirred solution of (E)-N-(1-((1R,5S)-3-((tert-butyldiphenylsilyl)oxy)bicyclo [3.1.0]hexan6-yl)ethylidene)-2-methylpropane-2-sulfinamide 66A (1.85 g, $3.84 \mathrm{mmol})$ in toluene $(38.4 \mathrm{~mL})$ at $80{ }^{\circ} \mathrm{C}$ was added trimethylaluminum $(2 \mathrm{M}$ in toluene) $(2.112 \mathrm{~mL}, 4.22 \mathrm{mmol})$ under nitrogen atmosphere. After 20 minutes, methyllithium $(5.28 \mathrm{~mL}, 8.45 \mathrm{mmol})$ was added dropwise and the mixture stirred at $80{ }^{\circ} \mathrm{C}$ for 4 hours. The reaction was quenched with $2 \mathrm{~mL}$ of water. The residue was adsorbed onto silica gel and purified via flash chromatography (0-80\% EtOAc in hexanes to give the title compound 66B (394 mg, $0.791 \mathrm{mmol}, 20.6 \%$ yield) as a yellow oil. $\mathrm{MS}\left(\mathrm{ES}^{+}\right)$ $\mathrm{C}_{29} \mathrm{H}_{43} \mathrm{NO}_{2} \mathrm{SSi}$ requires: 497, found: $498[\mathrm{M}+\mathrm{H}]^{+}$.

Step 4: 2-((1R,5S)-3-((tert-butyldiphenylsilyl)oxy)bicyclo[3.1.0]hexan-6-yl)propan-2-amine 66C.

To a solution of (S)-N-(2-((1R,5S,6r)-3-((tert-butyldiphenylsilyl)oxy)bicyclo[3.1.0]hexan-6yl)propan-2-yl)-2-methylpropane-2-sulfinamide 66B (1.9 g, $3.8 \mathrm{mmol})$ in $\mathrm{MeOH}(38 \mathrm{ml})$ at $0{ }^{\circ} \mathrm{C}$ was added $\mathrm{HCl}$ in dioxane $(4 \mathrm{M}, 1.4 \mathrm{ml}, 5.7 \mathrm{mmol})$ and the resulting mixture was stirred at room temperature for $3 \mathrm{~h}$. The reaction was quenched by addition of TEA (1.1 ml, 7.6 mmol). The volatiles were removed under reduced pressure and the title compound (1.5 g, $3.8 \mathrm{mmol}, 100 \%$ assumed) used as is in next reaction. $\mathrm{MS}\left(\mathrm{ES}^{+}\right) \mathrm{C}_{25} \mathrm{H}_{35} \mathrm{NOSi}$ requires: 393, found: $394[\mathrm{M}+\mathrm{H}]^{+}$. Step 5: N-(2-((1R,5S)-3-((tert-butyldiphenylsilyl)oxy)bicyclo[3.1.0]hexan-6-yl)propan-2-yl)-4chlorobenzamide 66D.

To a solution of 2-((1R,5S,6r)-3-((tert-butyldiphenylsilyl)oxy)bicyclo[3.1.0]hexan-6-yl)propan2-amine 66C (1.5 g, $3.8 \mathrm{mmol})$ and 4-chlorobenzoic acid (0.656 g, $4.19 \mathrm{mmol})$ in DMF (7.62 $\mathrm{ml})$ at $0{ }^{\circ} \mathrm{C}$ was added HATU $(1.9 \mathrm{~g}, 5.0 \mathrm{mmol})$ and TEA $(0.80 \mathrm{ml}, 5.7 \mathrm{mmol})$ and the resulting mixture was stirred at room temperature for 3 days. The reaction mixture was diluted with EtOAc $(30 \mathrm{~mL})$ and washed with $1 / 2$ sat $\mathrm{NaHCO}_{3}(30 \mathrm{~mL})$. The layers were separated, and the organic layer was washed with $\mathrm{H}_{2} \mathrm{O}(15 \mathrm{~mL})$, sat. $\mathrm{NaCl}(15 \mathrm{~mL})$, dried over $\mathrm{MgSO}_{4}$, filtered and concentrated under reduced pressure. The residue was purified by flash chromatography (0$100 \%$ EtOAc in hexanes) to give the title compound 66D (1.7 g $3.3 \mathrm{mmol}, 86 \%)$ and taken directly to the next step.

Step 6: 4-chloro-N-(2-((1R,3r,5S,6r)-3-hydroxybicyclo[3.1.0]hexan-6-yl)propan-2-yl)benzamide 66E. 
To a solution of $\mathrm{N}-(2-((1 \mathrm{R}, 5 \mathrm{~S}, 6 \mathrm{r})-3-(($ tert-butyldiphenylsilyl)oxy)bicyclo[3.1.0]hexan-6yl)propan-2-yl)-4-chlorobenzamide 66D $(1.7 \mathrm{~g}, 3.19 \mathrm{mmol})$ in THF $(3.19 \mathrm{~mL})$ at $0{ }^{\circ} \mathrm{C}$ was added TBAF in THF (3.83 mL, $3.83 \mathrm{mmol})$ and the resulting mixture was stirred at RT for $3 \mathrm{~h}$. The volatiles were removed under reduced pressure. The residue was purified by flash silica gel chromatography (0-100\% EtOAc in hexanes) to give the title compound 66E (284 mg, 0.967 mmol, $30 \%$ yield) as a yellow liquid (first diastereomer to elute). $\mathrm{MS}\left(\mathrm{ES}^{+}\right) \mathrm{C}_{16} \mathrm{H}_{20} \mathrm{ClNO}_{2}$ requires: 293, found: $294[\mathrm{M}+\mathrm{H}]^{+}$.

Step $\quad 7: \quad(1 R, 3 r, 5 S, 6 r)-6-(2-(4-c h l o r o b e n z a m i d o) p r o p a n-2-y l) b i c y c l o[3.1 .0]$ hexan-3-yl methanesulfonate 66F.

The title compound (354 $\mathrm{mg}, 0.95 \mathrm{mmol}, 100 \%$ ) as a light brown oil was sythesized similar to compound 79Et. $\mathrm{MS}\left(\mathrm{ES}^{+}\right) \mathrm{C}_{17} \mathrm{H}_{22} \mathrm{ClNO}_{4} \mathrm{~S}$ requires: 371, found: $372[\mathrm{M}+\mathrm{H}]^{+}$.

Step 8: Compound 66: 4-chloro-N-(2-((1R,3s,5S,6r)-3-(5,6-difluoro-1H-benzo[d]imidazol-1yl)bicyclo[3.1.0]hexan-6-yl)propan-2-yl)benzamide.

To a solution of (1R,3r,5S,6r)-6-(2-(4-chlorobenzamido)propan-2-yl)bicyclo[3.1.0]hexan-3-yl methanesulfonate 66F (350 mg, $0.941 \mathrm{mmol})$ in THF $(4.7 \mathrm{~mL}$ ) were added cesium carbonate (307 $\mathrm{mg}, 0.941 \mathrm{mmol})$ and 5,6-difluoro-1H-benzo[d]imidazole $(174 \mathrm{mg}, 1.13 \mathrm{mmol})$ and the resulting mixture was stirred at $65{ }^{\circ} \mathrm{C}$ for $15 \mathrm{~h}$. The reaction mixture was filtered through Celite, and the filtrate was concentrated under reduced pressure. The residue was purified by flash silica gel chromatography $\left(0-10 \% \mathrm{MeOH}\right.$ in $\mathrm{DCM}$ w/ $\left.0.5 \% \mathrm{NH}_{4} \mathrm{OH}\right)$ and then $(0-100 \%$ EtOAc in hexanes) to give the title compound ( $208 \mathrm{mg}, 0.48 \mathrm{mmol}, 51 \%$ yield) as a pale yellow powder after lyophilization. $\mathrm{MS}\left(\mathrm{ES}^{+}\right) \mathrm{C}_{23} \mathrm{H}_{22} \mathrm{ClF}_{2} \mathrm{~N}_{3} \mathrm{O}$ requires: 429 , found: $430[\mathrm{M}+\mathrm{H}]^{+}$. ${ }^{1} \mathrm{H} \mathrm{NMR}(600 \mathrm{MHz}$, $\left.\mathrm{CDCl}_{3}\right) \delta 7.91(\mathrm{~s}, 1 \mathrm{H}), 7.68(\mathrm{~d}, J=8.6 \mathrm{~Hz}, 2 \mathrm{H}), 7.55(\mathrm{dd}, J=10.9,7.3 \mathrm{~Hz}, 1 \mathrm{H}), 7.40(\mathrm{~d}, J=8.4$ $\mathrm{Hz}, 2 \mathrm{H}), 7.18$ (dd, $J=11.2,7.4 \mathrm{~Hz}, 1 \mathrm{H}), 4.55-4.40$ (m, 1H), $2.48-2.38(\mathrm{~m}, 2 \mathrm{H}), 2.35-2.25$ (m, $2 \mathrm{H}), 1.68-1.62(\mathrm{~m}, 2 \mathrm{H}), 1.55-1.50(\mathrm{~m}, 1 \mathrm{H}), 1.35(5,6 \mathrm{H})$.

Compound 67: 4-chloro-N-((R)-cyclopropyl((1R,3S,5S,6r)-3-(5,6-difluoro-1Hbenzo[d]imidazol-1-yl)bicyclo[3.1.0]hexan-6-yl)methyl)benzamide. 

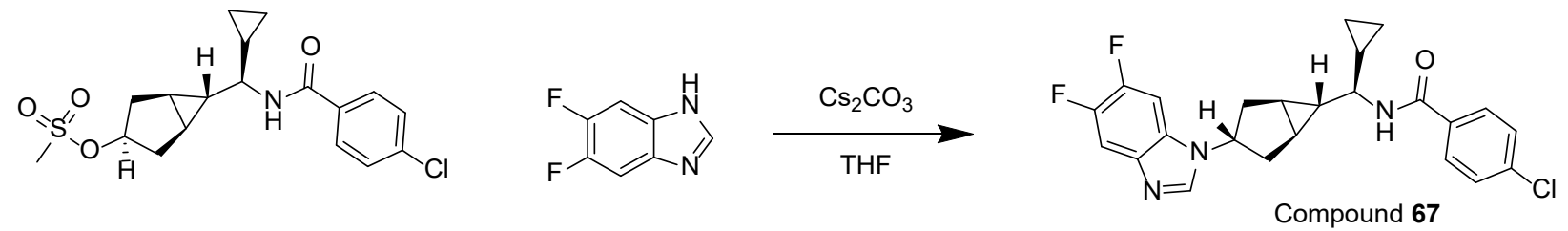

The title compound was synthesized similar to compound 62 from (1R,3R,5S,6r)-6-((R)-(4chlorobenzamido)(cyclopropyl)methyl)bicyclo[3.1.0] hexan-3-yl methanesulfonate (synthesized similar to $79 \mathrm{Et}(\mathbf{R})$. MS $\left(\mathrm{ES}^{+}\right) \mathrm{C}_{24} \mathrm{H}_{22} \mathrm{ClF}_{2} \mathrm{~N}_{3} \mathrm{O}$ requires: 441 , found: $442[\mathrm{M}+\mathrm{H}]^{+}$. ${ }^{1} \mathrm{H} \mathrm{NMR}$ (600 MHz, DMSO) $\delta 8.49$ (d, $J=8.6 \mathrm{~Hz}, 1 \mathrm{H}), 8.43(\mathrm{~s}, 1 \mathrm{H}), 7.90(\mathrm{~d}, J=8.3 \mathrm{~Hz}, 2 \mathrm{H}), 7.88-7.82$ (m, 1H), $7.69(\mathrm{dd}, J=11.1,7.4 \mathrm{~Hz}, 1 \mathrm{H}), 7.55(\mathrm{~d}, J=8.4 \mathrm{~Hz}, 2 \mathrm{H}), 4.70-4.58(\mathrm{~m}, 1 \mathrm{H}), 3.21-$ $3.13(\mathrm{~m}, 1 \mathrm{H}), 2.40-2.13(\mathrm{~m}, 4 \mathrm{H}), 1.54-1.43(\mathrm{~m}, 2 \mathrm{H}), 1.32-1.22(\mathrm{~m}, 1 \mathrm{H}), 1.15-1.07$ (m, $1 \mathrm{H}), 0.58-0.48(\mathrm{~m}, 1 \mathrm{H}), 0.43-0.34(\mathrm{~m}, 2 \mathrm{H}), 0.31-0.22(\mathrm{~m}, 1 \mathrm{H})$.

Compound 68: 4-cyano-N-((R)-1-((1R,3S,5S,6r)-3-(5,6-difluoro-1H-benzo[d]imidazol-1yl)bicyclo[3.1.0]hexan-6-yl)ethyl)benzamide
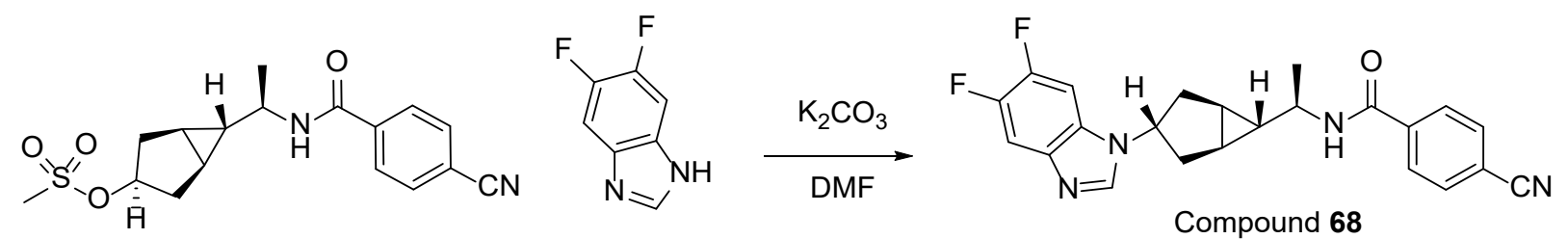

The title compound was synthesized similar to compounds $\mathbf{3 1}$ and $\mathbf{6 2}$ from (1R,3R,5S,6r)-6-((R)1-(4-cyanobenzamido)ethyl)bicyclo[3.1.0]hexan-3-yl methanesulfonate (synthesized similar to 79Et(R)). MS (ES $\left.{ }^{+}\right) \mathrm{C}_{23} \mathrm{H}_{20} \mathrm{~F}_{2} \mathrm{~N}_{4} \mathrm{O}$ requires: 406, found: $407[\mathrm{M}+\mathrm{H}]^{+}$. ${ }^{1} \mathrm{H}$ NMR $(600 \mathrm{MHz}$, DMSO- $\left.d_{6}\right) \delta 8.62(\mathrm{~d}, J=8.0 \mathrm{~Hz}, 1 \mathrm{H}), 8.43(\mathrm{~s}, 1 \mathrm{H}), 8.02(\mathrm{~d}, J=8.1 \mathrm{~Hz}, 2 \mathrm{H}), 7.97(\mathrm{~d}, J=8.2 \mathrm{~Hz}$, 2H), $7.83(\mathrm{dd}, J=10.9,7.3 \mathrm{~Hz}, 1 \mathrm{H}), 7.69$ (dd, $J=11.1,7.4 \mathrm{~Hz}, 1 \mathrm{H}), 4.60$ (p, $J=9.3 \mathrm{~Hz}, 1 \mathrm{H}), 3.60$ (q, $J=7.5 \mathrm{~Hz}, 1 \mathrm{H}), 2.34$ (dd, $J=12.4,7.6 \mathrm{~Hz}, 1 \mathrm{H}), 2.27$ (dd, $J=12.5,7.7 \mathrm{~Hz}, 1 \mathrm{H}), 2.25-2.13$ $(\mathrm{m}, 2 \mathrm{H}), 1.52(\mathrm{~s}, 1 \mathrm{H}), 1.41(\mathrm{~s}, 1 \mathrm{H}), 1.26(\mathrm{~d}, J=6.7 \mathrm{~Hz}, 3 \mathrm{H}), 1.19(\mathrm{~m}, 1 \mathrm{H})$.

Compound $\quad 69: \quad$ N-((R)-1-((1R,3S,5S,6r)-3-(5,6-difluoro-1H-benzo[d]imidazol-1yl)bicyclo[3.1.0]hexan-6-yl)propyl)-3-fluorobicyclo[1.1.1]pentane-1-carboxamide. 


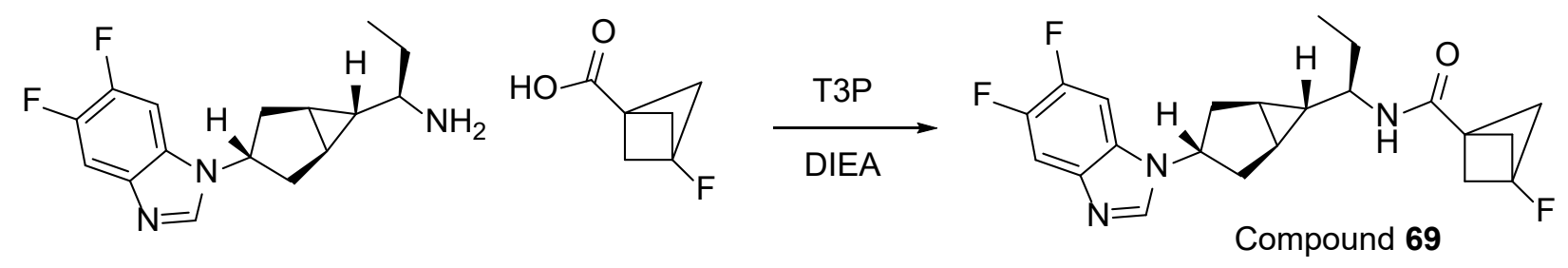

To a cooled $0{ }^{\circ} \mathrm{C}$ solution of (R)-1-((1R,3S,5S,6r)-3-(5,6-difluoro-1H-benzo[d]imidazol-1yl)bicyclo[3.1.0] hexan-6-yl)propan-1-amine (amine 63D from synthesis of compound 63) (19 $\mathrm{mg}, 0.065 \mathrm{mmol})$ in THF (217 $\mu \mathrm{l})$ were added DIEA (34.2 $\mu 1,0.196 \mathrm{mmol})$ and 3fluorobicyclo[1.1.1]pentane-1-carboxylic acid $(9.9 \mathrm{mg}, 0.072 \mathrm{mmol})$ and the resulting mixture was stirred at $0{ }^{\circ} \mathrm{C}$ for $5 \mathrm{~min}$. To the reaction at $0{ }^{\circ} \mathrm{C}$ was added T3P $(50 \%$ wt in EtOAc, $116 \mu 1$, $0.196 \mathrm{mmol}$ ) the reaction was stirred at $0{ }^{\circ} \mathrm{C}$ for $5 \mathrm{~min}$ then at room temperature overnight. The reaction was diluted with EtOAc, washed with water and brine. The aqueous layer was extracted with EtOAc once. The organic layers combined, dried over $\mathrm{MgSO}_{4}$, filtered, concentrated, and purified by flash chromatography (10-70\% 80:20:2 EtOAc:EtOH:NH4OH in hexanes) to give the title compound (4 mg, $0.010 \mathrm{mmol}, 15 \%$ yield) as a white solid. $\mathrm{MS}\left(\mathrm{ES}^{+}\right) \mathrm{C}_{22} \mathrm{H}_{24} \mathrm{~F}_{3} \mathrm{~N}_{3} \mathrm{O}$ requires: 403, found: $404[\mathrm{M}+\mathrm{H}]^{+}$. ${ }^{1} \mathrm{H}$ NMR $(600 \mathrm{MHz}, \mathrm{DMSO}) \delta 8.43$ (s, 1H), 7.83 (dd, $J=$ 10.9, 7.3 Hz, 1H), $7.69(\mathrm{dd}, J=11.1,7.5 \mathrm{~Hz}, 1 \mathrm{H}), 7.64(\mathrm{~d}, J=8.7 \mathrm{~Hz}, 1 \mathrm{H}), 4.63-4.52(\mathrm{~m}, 1 \mathrm{H})$, $3.24-3.14(\mathrm{~m}, 1 \mathrm{H}), 2.34-2.09$ (m, 10H), $1.62-1.46(\mathrm{~m}, 2 \mathrm{H}), 1.43-1.27(\mathrm{~m}, 2 \mathrm{H}), 1.02(\mathrm{dt}, J$ $=7.7,3.3 \mathrm{~Hz}, 1 \mathrm{H}), 0.85(\mathrm{t}, J=7.4 \mathrm{~Hz}, 3 \mathrm{H})$.

\section{Compound 72: 6-fluoro-4-(((1R,3s,5S,6r)-6-(1-(5-(trifluoromethyl)-1H-benzo[d]imidazol-2-} yl)propyl)bicyclo[3.1.0]hexan-3-yl)oxy)quinoline 


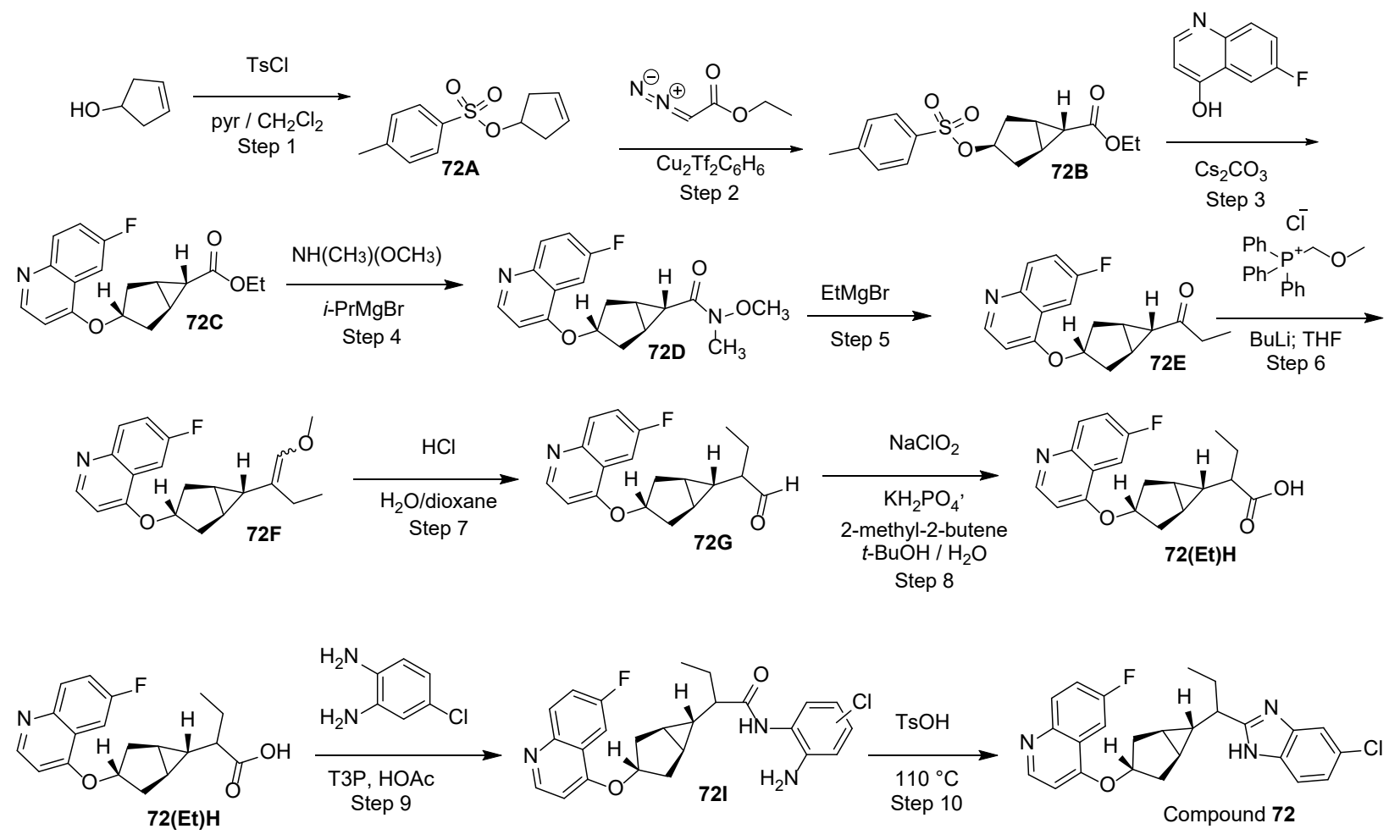

Step 1: cyclopent-3-en-1-yl 4-methylbenzenesulfonate 72A. To a solution of cyclopent-3-ene-1-ol $(70.2 \mathrm{~g}, 835 \mathrm{mmol})$ in pyridine $(150 \mathrm{~mL})$ and DCM $(300 \mathrm{ml})$ cooled at $4{ }^{\circ} \mathrm{C}$ under $\mathrm{N}_{2}$ atm was added slowly with stirring 4-toluene-sulfonyl chloride (175 g, $918 \mathrm{mmol})$. The mixture was then stirred under $\mathrm{N}_{2}$ atm at this temperature for $1 \mathrm{~h}$, then stood in cold room at $4^{\circ} \mathrm{C}$ for $48 \mathrm{~h}$. Most of the dichloromethane was evaporated at reduced pressure. The mixture was diluted with EtOAc/hexanes $(20 \%, 600 \mathrm{ml})$ and washed with water $(5 \mathrm{x})$ and brine. The aqueous phases were back extracted with EtOAc/hexanes $(20 \%, 1$ x $400 \mathrm{ml})$, the organic layers combined, dried over $\mathrm{MgSO}_{4}$, and then filtered through a short plug $(3 \mathrm{~cm} \times 11 \mathrm{~cm})$ of silica (washed with a further $1000 \mathrm{ml} \mathrm{20 \%} \mathrm{EtOAc/Hexane).} \mathrm{The} \mathrm{solvent} \mathrm{was} \mathrm{evaporated} \mathrm{at} \mathrm{reduced} \mathrm{pressure} \mathrm{to} \mathrm{give} \mathrm{a}$ colorless oil. The residual solvent in the crude product was azeotroped with hexanes then twice with toluene. The resulting colorless oil was further dried by azeotroping once with hexanes and then crystallizing by diluting with an equal volume of hexane and heating at $50^{\circ} \mathrm{C}$. The resulting biphasic mixture of colorless oil and solids in hexanes was allowed to age at RT overnight. The solid was removed by filtration and washed with cold hexanes and then dried in-vacuum to give the title compound 72A (173 g, $727 \mathrm{mmol}, 87 \%)$. ${ }^{1} \mathrm{H}$ NMR (CHLOROFORM-d) d: 7.79 (d, J = $7.9 \mathrm{~Hz}, 2 \mathrm{H}), 7.34$ (d, J = 7.9 Hz, 2H), 5.65 (s, 2H), 5.11 - $5.24(\mathrm{~m}, 1 \mathrm{H}), 2.59$ - 2.67 (m, 2H), 2.48 - $2.55(\mathrm{~m}, 2 \mathrm{H}), 2.45(\mathrm{~s}, 3 \mathrm{H})$. 
Step 2: ethyl (1R,3r,5S,6s)-3-(tosyloxy)bicyclo[3.1.0]hexane-6-carboxylate 72B. To a flask containing the product from the previous step 72A $(60.45 \mathrm{~g}, 254 \mathrm{mmol})$ in DCM $(100 \mathrm{~mL})$ was added, under nitrogen, a solution of copper(I) triflate.0.5 benzene complex (1.03 g) and (4S,4'S)2,2'-(propane-2,2-diyl)bis(4-isopropyl-4,5-dihydrooxazole) $(1 \mathrm{ml})$ in DCM (10 mL) prepared under $\mathrm{N}_{2}$ and ultrasonication to give a green solution. Ethyl diazoacetate $(20 \mathrm{~mL}$ containing 13 $20 \mathrm{wt} \% \mathrm{DCM}$ and diluted 1:0.5 with DCM) was added dropwise via syringe pump at a rate of 9 $\mathrm{mL} / \mathrm{h}$ over $\sim 3.5 \mathrm{~h}$. A further $30 \mathrm{~mL}$ of ethyl diazoacetate (undiluted) was then added at a rate of 8 $\mathrm{mL} / \mathrm{h}$ (for the first $12 \mathrm{~mL}$ added) then slowed to $4 \mathrm{~mL} / \mathrm{h}$ (for remaining $18 \mathrm{ml}$ ), with provision for venting the $\mathrm{N}_{2}$ gas that was generated by the reaction. Following completion of addition, the reaction was stirred overnight. To the reaction was then added ethyl diazoacetate $(20 \mathrm{~mL}$ containing 13-20 wt \%DCM and diluted 1:0.5 with DCM) at a rate of $4 \mathrm{~mL} / \mathrm{h}$, then again stirred RT overnight. Total quantity of ethyl diazoacetate added was $70 \mathrm{~mL}$ containing $13-20 \mathrm{wt} \%$ DCM. The solvent was then removed at reduced pressure, the residue taken up in $10 \%$ EtOAc/Hexanes, passed through pad of silica gel $(15 \mathrm{~cm}$ high x $10.5 \mathrm{~cm}$ diameter $)$. The diethyl fumarate (or maleate) (colorless liquid) was eluted from the plug with 10\% EtOAc/Hexanes. A mixture of fumarate and starting olefin (colorless oil $21 \mathrm{~g}$ ) followed by a mixture of isomers of the desired product were eluted with $20 \%$ EtOAc/hexanes. The desired isomer of the product was crystalized from $\mathrm{Et}_{2} \mathrm{O} / \mathrm{Hexane}$ to give the title compound $\mathbf{7 2 B}$ (41 g, $\left.126 \mathrm{mmol}, 50 \%\right)$ as colorless crystalline solid (rods). If desired, the product can be further purified by flash chromatography. ${ }^{1} \mathrm{H}$ NMR (600 MHz, DMSO-d $\left.{ }_{6}\right) \delta$ ppm 1.10 - 1.20 (m, 3 H) $1.52-1.58$ (m, 1 H) 1.71 - $1.78(\mathrm{~m}, 2 \mathrm{H}) 1.82$ - $1.91(\mathrm{~m}, 2 \mathrm{H}) 2.03$ - $2.13(\mathrm{~m}, 2 \mathrm{H}) 2.38$ - 2.45 (m, 3 H) 3.93 - 4.04 (m, 2 H) 4.60 - $4.74(\mathrm{~m}, 1 \mathrm{H}) 7.42$ - 7.55 (m, 2 H) 7.73 - 7.85 (m, 2 H).

Step 3: ethyl (1R,3s,5S,6r)-3-((6-fluoroquinolin-4-yl)oxy)bicyclo[3.1.0]hexane-6-carboxylate 72C. To a solution of the product from the previous step72B (8.0 g, $24.66 \mathrm{mmol})$ in NMP $(40 \mathrm{ml})$ were added 6-fluoroquinolin-4-ol (4.22 g, $25.9 \mathrm{mmol})$ and $\mathrm{Cs}_{2} \mathrm{CO}_{3}(9.64 \mathrm{~g}, 29.6 \mathrm{mmol})$ and the resulting mixture was stirred at RT 3 days under $\mathrm{N}_{2}$. The solution was added to ice / cold water and stirred until ice melted and brown precipitate formed. The mixture was filtered, the solid washed with ice / cold water, and dried under vacuum to give the title compound $\mathbf{7 2 C}$ (5.86 g, $18.6 \mathrm{mmol}, 75 \%)$. MS (ES ${ }^{+} \mathrm{C}_{18} \mathrm{H}_{18} \mathrm{FNO}_{3}$ requires: 315, found: $316[\mathrm{M}+\mathrm{H}]^{+}$. ${ }^{1} \mathrm{H}$ NMR 
$($ CHLOROFORM- $d) \delta 8.69(\mathrm{~d}, \mathrm{~J}=4.9 \mathrm{~Hz}, 1 \mathrm{H}), 7.96-8.04(\mathrm{~m}, 1 \mathrm{H}), 7.77(\mathrm{~d}, \mathrm{~J}=9.1 \mathrm{~Hz}, 1 \mathrm{H})$, $7.64(\mathrm{t}, \mathrm{J}=8.3 \mathrm{~Hz}, 1 \mathrm{H}), 7.12(\mathrm{~d}, \mathrm{~J}=4.9 \mathrm{~Hz}, 1 \mathrm{H}), 4.93$ (quin, $\mathrm{J}=7.1 \mathrm{~Hz}, 1 \mathrm{H}), 4.06$ (q, J = 7.1 Hz, 1H), 2.58 (dd, J =12.8, 7.2 Hz, 1H), 2.00 - 2.11 (m, 1H), 1.92 (br. s., 1H), 1.69 (br. s., 1H), 1.20 $(\mathrm{t}, \mathrm{J}=7.0 \mathrm{~Hz}, 1 \mathrm{H})$.

General Procedure for Weinreb amide synthesis.

Step 4: (1R,3s,5S,6r)-3-((6-Fluoroquinolin-4-yl)oxy)-N-methoxy-N-methylbicyclo[3.1.0]hexane6-carboxamide 72D. To a suspension of the product from the previous step 72C (3.97 g, 12.6 mmol) in THF (21 ml) was added $\mathrm{N}, O$-dimethylhydroxylamine hydrochloride $(2.46 \mathrm{~g}, 25.2$ $\mathrm{mmol}$ ) and the resulting mixture was sonicated for $1 \mathrm{~min}$ and stirred at RT for $5 \mathrm{~min}$ then cooled to $-13^{\circ} \mathrm{C}$ in an acetone ice bath. To the cooled reaction was added dropwise over $20 \mathrm{~min}$, isopropyl magnesium chloride $(2 \mathrm{M}, 34.6 \mathrm{ml}, 69.2 \mathrm{mmol})$ and during the addition the temp was kept between -10 to $-15^{\circ} \mathrm{C}$. After the addition the reaction was stirred at -10 to $-15^{\circ} \mathrm{C}$ for $1 \mathrm{hr}$. The reaction was slowly and portion wise poured into a stirring mixture of ice and saturated $\mathrm{NH}_{4} \mathrm{Cl}$ (500 ml, total). The mixture was stirred for $1 \mathrm{~h}$ and then extracted with EtOAc/DCM mixture $(200 / 100 \mathrm{ml})$ and DCM $(2 \times 200 \mathrm{ml})$. The organic layers combined, dried over $\mathrm{MgSO}_{4}$, filtered, and concentrated to give the title compound 72D (3.89 g, $11.8 \mathrm{mmol}, 94 \%$ yield $)$ as a white-to-light yellow solid. ${ }^{1} \mathrm{H}$ NMR (600 MHz, DMSO-d $\left.d_{6}\right) \delta 8.70$ (d, $\left.J=5.2 \mathrm{~Hz}, 1 \mathrm{H}\right), 8.01$ (dd, $J=9.3,5.3 \mathrm{~Hz}, 1 \mathrm{H}), 7.78(\mathrm{dd}, J=9.8,3.0 \mathrm{~Hz}, 1 \mathrm{H}), 7.68-7.62(\mathrm{~m}, 1 \mathrm{H}), 7.15(\mathrm{~d}, J=5.3 \mathrm{~Hz}$, 1H), 5.00 (p, $J=7.1 \mathrm{~Hz}, 1 \mathrm{H}), 3.75(\mathrm{~s}, 3 \mathrm{H}), 3.12$ (s, 3H), 2.58 (dd, $J=13.3,7.2 \mathrm{~Hz}, 2 \mathrm{H}), 2.13-$ $2.00(\mathrm{~m}, 3 \mathrm{H}), 1.90-1.78(\mathrm{~m}, 2 \mathrm{H}) . \mathrm{MS}\left(\mathrm{ES}^{+}\right) \mathrm{C}_{18} \mathrm{H}_{19} \mathrm{FN}_{2} \mathrm{O}_{3}$ requires: 330 , found: $331[\mathrm{M}+\mathrm{H}]^{+}$.

General Procedure for synthesis of a alkyl ketone from a Weinreb amide.

Step 5: 1-((1R,3s,5S,6r)-3-((6-fluoroquinolin-4-yl)oxy)bicyclo[3.1.0]hexan-6-yl)propan-1-one 72E. To a cooled $-78{ }^{\circ} \mathrm{C}$ solution of the product from the previous step $(4.24 \mathrm{~g}, 12.8 \mathrm{mmol})$ in THF $(51 \mathrm{ml})$ was added ethyl magnesium bromide $(7.7 \mathrm{ml}, 19.3 \mathrm{mmol})$ dropwise and the resulting mixture was stirred at $-78^{\circ} \mathrm{C}$ for $15 \mathrm{~min}$, then at $\mathrm{RT}$ overnight. The reaction was poured into mixture of ice and saturated $\mathrm{NH}_{4} \mathrm{Cl}$ and stirred until ice melted. The mixture was extracted with EtOAc $(3 \times 200 \mathrm{ml})$. The organic layers were washed with brine, combined, dried over MgSO4, filtered, and concentrated to give the title compound 72E (3.59 g, 12 mmol, 93\% yield). MS (ES $\left.{ }^{+}\right) \mathrm{C}_{18} \mathrm{H}_{18} \mathrm{FNO}_{2}$ requires: 299, found: $300[\mathrm{M}+\mathrm{H}]^{+} .{ }^{1} \mathrm{H}$ NMR (500 MHz, DMSO) $\delta 8.70$ 
$(\mathrm{d}, J=5.2 \mathrm{~Hz}, 1 \mathrm{H}), 8.01(\mathrm{dd}, J=9.2,5.4 \mathrm{~Hz}, 1 \mathrm{H}), 7.77(\mathrm{dd}, J=9.7,2.9 \mathrm{~Hz}, 1 \mathrm{H}), 7.68-7.60$ (m, $1 \mathrm{H}), 7.11(\mathrm{~d}, J=5.1 \mathrm{~Hz}, 1 \mathrm{H}), 4.96(\mathrm{p}, J=7.3 \mathrm{~Hz}, 1 \mathrm{H}), 2.61-2.52(\mathrm{~m}, 4 \mathrm{H}), 2.09-2.01(\mathrm{~m}, 3 \mathrm{H})$, $1.92-1.88(\mathrm{~m}, 2 \mathrm{H}), 0.96(\mathrm{t}, J=7.3 \mathrm{~Hz}, 3 \mathrm{H})$.

General Procedure for Wittig synthesis of a vinyl ether.

Step 6: 6-fluoro-4-(((1R,3s,5S,6r)-6-(1-methoxybut-1-en-2-yl)bicyclo[3.1.0]hexan-3-

yl)oxy)quinoline $\mathbf{7 2 F}$. To a cooled $-78^{\circ} \mathrm{C}$ solution of (methoxymethyl)triphenylphosphonium chloride (6.58 g, $19.2 \mathrm{mmol})$ in THF (30 ml) was added n-butyllithium (2.5M in THF, $7.2 \mathrm{ml}, 18$ mmol) dropwise over $15 \mathrm{~min}$. The reaction was stirred at $-78^{\circ} \mathrm{C}$ for $30 \mathrm{~min}$ and then at $0^{\circ} \mathrm{C}$ for $30 \mathrm{~min}$. To the reaction was added the product from the previous step 72E $(3.6 \mathrm{~g}, 12 \mathrm{mmol})$ dissolved in THF $(10 \mathrm{ml})$, and the reaction was stirred at $0{ }^{\circ} \mathrm{C}$ for $10 \mathrm{~min}$ then at $\mathrm{RT}$. The reaction was poured into a mixture of ice and saturated $\mathrm{NH}_{4} \mathrm{Cl}(300 \mathrm{ml})$ and the resulting mixture was extracted with EtOAc $(3 \times 150 \mathrm{ml})$. The organic layers were washed with brine, combined, dried over $\mathrm{MgSO}_{4}$, filtered, concentrated, and purified by flash chromatography (30 to $100 \%$ EtOAc in Hexanes) to give the title compound 72F (3.1 g, $9.5 \mathrm{mmol}, 79 \%$ yield) as a yellow, viscous oil. $\mathrm{MS}\left(\mathrm{ES}^{+}\right) \mathrm{C}_{20} \mathrm{H}_{22} \mathrm{FNO}_{2}$ requires: 327, found: $328[\mathrm{M}+\mathrm{H}]^{+}$. ${ }^{1} \mathrm{H} \mathrm{NMR}(600 \mathrm{MHz}$, DMSO) $\delta 8.68(\mathrm{t}, J=5.2 \mathrm{~Hz}, 1 \mathrm{H}), 8.00(\mathrm{dd}, J=9.2,5.4 \mathrm{~Hz}, 1 \mathrm{H}), 7.76(\mathrm{dd}, J=9.7,3.0 \mathrm{~Hz}, 1 \mathrm{H})$, $7.66-7.60(\mathrm{~m}, 1 \mathrm{H}), 7.11(\mathrm{t}, J=5.6 \mathrm{~Hz}, 1 \mathrm{H}), 5.89-5.71(\mathrm{~m}, 1 \mathrm{H}), 4.96-4.87(\mathrm{~m}, 1 \mathrm{H}), 3.49(\mathrm{~d}, J$ $=20.7 \mathrm{~Hz}, 3 \mathrm{H}), 2.55-2.46(\mathrm{~m}, 2 \mathrm{H}), 2.06-1.94(\mathrm{~m}, 3 \mathrm{H}), 1.71-1.65(\mathrm{~m}, 1 \mathrm{H}), 1.62-1.57(\mathrm{~m}$, $1 \mathrm{H}), 1.45-1.41(\mathrm{~m}, 1 \mathrm{H}), 1.38-1.05(\mathrm{~m}, 1 \mathrm{H}), 1.00-0.92(\mathrm{~m}, 3 \mathrm{H})$.

General Procedure for hydrolysis of the vinyl ether.

Step 7: 2-((1R,3s,5S,6r)-3-((6-fluoroquinolin-4-yl)oxy)bicyclo[3.1.0]hexan-6-yl)butanal 72G. To a solution of the product from the previous step 72F $(3.1 \mathrm{~g}, 9.5 \mathrm{mmol})$ in THF $(38 \mathrm{ml})$ and $\mathrm{H}_{2} \mathrm{O}$ $(9 \mathrm{ml})$ was added $\mathrm{HCl}(4 \mathrm{M}$ in dioxane, $4.7 \mathrm{ml}, 19 \mathrm{mmol})$ and the resulting mixture was stirred at $70{ }^{\circ} \mathrm{C}$ for $2 \mathrm{~h}$. The reaction was diluted with EtOAc $(150 \mathrm{ml})$ and water $(250 \mathrm{ml})$ and the mixture neutralized with saturated $\mathrm{NaHCO}_{3}$. The resulting mixture was mixed, the organic layer separated, and the aqueous layer extracted with EtOAc $(2 \times 150 \mathrm{ml})$. The organic layers were washed with brine, combined, dried over $\mathrm{MgSO}_{4}$, filtered, concentrated, dissolved in minimal DCM and purified by flash chromatography 72G (30-100\% EtOAc in Hexanes) to give the title compound (2.9 g, 9.4 mmol, 99\% yield). MS (ES ${ }^{+} \mathrm{C}_{19} \mathrm{H}_{20} \mathrm{FNO}_{2}$ requires: 313, found: 314 
$[\mathrm{M}+\mathrm{H}]^{+} .{ }^{1} \mathrm{H}$ NMR $(600 \mathrm{MHz}, \mathrm{DMSO}) \delta 9.64(\mathrm{~d}, J=2.1 \mathrm{~Hz}, 1 \mathrm{H}), 8.68(\mathrm{~d}, J=5.2 \mathrm{~Hz}, 1 \mathrm{H}), 8.00$ $(\mathrm{dd}, J=9.2,5.4 \mathrm{~Hz}, 1 \mathrm{H}), 7.75(\mathrm{dd}, J=9.8,3.0 \mathrm{~Hz}, 1 \mathrm{H}), 7.67-7.61(\mathrm{~m}, 1 \mathrm{H}), 7.10(\mathrm{~d}, J=5.3 \mathrm{~Hz}$, $1 \mathrm{H}), 4.89$ (p, $J=7.2 \mathrm{~Hz}, 1 \mathrm{H}), 2.55-2.46(\mathrm{~m}, 2 \mathrm{H}), 2.05-1.94(\mathrm{~m}, 2 \mathrm{H}), 1.72-1.64(\mathrm{~m}, 1 \mathrm{H})$, $1.61-1.48(\mathrm{~m}, 2 \mathrm{H}), 1.46-1.34(\mathrm{~m}, 2 \mathrm{H}), 0.92(\mathrm{t}, J=7.3 \mathrm{~Hz}, 3 \mathrm{H}), 0.67-0.62(\mathrm{~m}, 1 \mathrm{H})$.

General Procedure for oxidation of an aldehyde.

Step 8: 2-((1R,3s,5S,6r)-3-((6-Fluoroquinolin-4-yl)oxy)bicyclo[3.1.0]hexan-6-yl)propanoic acid 72(Et)H To a cooled $0^{\circ} \mathrm{C}$ solution of the product from the previous step 72G $(2.9 \mathrm{~g}, 9.4$ $\mathrm{mmol})$ in $t$ - $\mathrm{BuOH}(35 \mathrm{ml})$ and 2-methyl-2-butene $(5 \mathrm{ml}, 47 \mathrm{mmol})$ was added dropwise a freshly prepared solution of $\mathrm{KH}_{2} \mathrm{PO}_{4}(2.5 \mathrm{~g}, 19 \mathrm{mmol})$ and $\mathrm{NaClO}_{2}(1.7 \mathrm{~g}, 19 \mathrm{mmol})$ in $\mathrm{H}_{2} \mathrm{O}(12 \mathrm{ml})$. The resulting mixture was stirred at $0^{\circ} \mathrm{C}$ for $0.5 \mathrm{~h}$, ice bath change and then allowed to warm to RT $4.5 \mathrm{~h}$. The reaction mixture was diluted with water $(400 \mathrm{ml})$, stirred for $30 \mathrm{~min}$, resulting precipitate filtered, the solid rinsed with water and hexanes and dried in vacuo to give the title compound 72(Et)H as a white solid (2.2 g $6.7 \mathrm{mmol}, 72 \%$ yield). The filtrate was acidified (pH 4) with $1 \mathrm{M} \mathrm{HCl}$, extracted with DCM (3 x $75 \mathrm{ml})$, the organic extracts combined, washed with brine, dried over $\mathrm{MgSO}_{4}$, filtered, and concentrated to give the desired product $(1 \mathrm{~g})$. The precipitate and the extracted solid were combined to give the title compound $(0.23 \mathrm{~g})$ as a white solid. MS (ES $\left.{ }^{+}\right) \mathrm{C}_{19} \mathrm{H}_{20} \mathrm{FNO}_{3}$ requires: 329, found: $330[\mathrm{M}+\mathrm{H}]^{+} .{ }^{1} \mathrm{H}$ NMR (600 MHz, DMSO-d $)$ $\delta 12.08(\mathrm{~s}, 1 \mathrm{H}), 8.67(\mathrm{~d}, J=5.2 \mathrm{~Hz}, 1 \mathrm{H}), 8.00(\mathrm{dd}, J=9.2,5.4 \mathrm{~Hz}, 1 \mathrm{H}), 7.74(\mathrm{dd}, J=9.8,3.0 \mathrm{~Hz}$, 1H), $7.67-7.59(\mathrm{~m}, 1 \mathrm{H}), 7.11(\mathrm{~d}, J=5.2 \mathrm{~Hz}, 1 \mathrm{H}), 4.89$ (p, J=7.2 Hz, 1H), $2.49-2.38(\mathrm{~m}, 2 \mathrm{H})$, $2.00-1.92(\mathrm{~m}, 2 \mathrm{H}), 1.69-1.53(\mathrm{~m}, 2 \mathrm{H}), 1.52-1.46(\mathrm{~m}, 1 \mathrm{H}), 1.38-1.27(\mathrm{~m}, 2 \mathrm{H}), 0.90(\mathrm{t}, J=$ $7.4 \mathrm{~Hz}, 3 \mathrm{H}), 0.78-0.72(\mathrm{~m}, 1 \mathrm{H})$.

Step 9: N-(2-amino-4/5-chlorophenyl)-2-((1R,3s,5S,6r)-3-((6-fluoroquinolin-4yl)oxy)bicyclo[3.1.0]hexan-6-yl)butanamide 72I. To a vial containing 72(Et)H (380 mg, 1.15 $\mathrm{mmol}$ ), and 4-chlorobenzene-1,2-diamine (329 mg, $2.31 \mathrm{mmol}$ ) was added THF (5.8 mL), pyridine $(86 \mu \mathrm{L}, 1.063 \mathrm{mmol})$ followed by PyBrop $(807 \mathrm{mg}, 1.73 \mathrm{mmol})$ and the resulting mixture was stirred at $70{ }^{\circ} \mathrm{C} 4 \mathrm{~h}$. The reaction was diluted with EtOAc and quenched with saturated $\mathrm{NaHCO}_{3}$. The layers separated, aqueous layer extracted with EtOAc (2 x), organic layers combined, washed with brine, dried over $\mathrm{MgSO}_{4}$, and concentrated to give crude title compound 72I (500 $\mathrm{mg}, 0.96 \mathrm{mmol})$ and used as is in the next step. 
Step 10: Compound 72: 4-(((1R,3s,5S,6r)-6-(1-(5-chloro-1H-benzo[d]imidazol-2yl)propyl)bicyclo[3.1.0]hexan-3-yl)oxy)-6-fluoroquinoline. The product from the previous step 72I was suspended in toluene $(5.8 \mathrm{~mL})$, pTsOH $(1.1 \mathrm{~g}, 6.4 \mathrm{mmol})$ was added and the reaction heated in a dry block at $130{ }^{\circ} \mathrm{C}$ for $4 \mathrm{~h}$. The reaction was diluted with EtOAc, washed with water, saturated $\mathrm{NaHCO}_{3}$ and brine. The aqueous layers were extracted with EtOAc $(1 \mathrm{x})$. The organic layers combined, dried over $\mathrm{Na}_{2} \mathrm{SO}_{4}$, decanted, concentrated and purified by flash chromatography (0-100\% of 90:10:1 DCM:MeOH: $\mathrm{NH}_{4} \mathrm{OH}$ in DCM) to give the title compound as a light brown solid (144 $\mathrm{mg}, 0.33 \mathrm{mmol}, 34 \%$ yield). $\mathrm{MS}\left(\mathrm{ES}^{+}\right) \mathrm{C}_{25} \mathrm{H}_{23} \mathrm{ClFN}_{3} \mathrm{O}$ requires: 435 , found: $436[\mathrm{M}+\mathrm{H}]^{+}$. ${ }^{1} \mathrm{H}$ NMR $(600 \mathrm{MHz}, \mathrm{DMSO}) \delta 12.30(\mathrm{~d}, J=23.0 \mathrm{~Hz}, 1 \mathrm{H}), 8.66(\mathrm{~d}, J=5.2$ Hz, 1H), 7.99 (dd, $J=9.1,5.4 \mathrm{~Hz}, 1 \mathrm{H}), 7.74$ (dd, $J=9.7,3.0 \mathrm{~Hz}, 1 \mathrm{H}), 7.65-7.54$ (m, 2H), 7.51 $-7.44(\mathrm{~m}, 1 \mathrm{H}), 7.19-7.12(\mathrm{~m}, 1 \mathrm{H}), 7.08(\mathrm{~d}, J=5.3 \mathrm{~Hz}, 1 \mathrm{H}), 4.91-4.83(\mathrm{~m}, 1 \mathrm{H}), 2.55$ (dd, $J=$ 13.0, 7.2 Hz, 1H), 2.35 (dd, $J=12.9,7.1 \mathrm{~Hz}, 1 \mathrm{H}), 2.19-2.13$ (m, 1H), $2.04-1.97$ (m, 1H), 1.95 $-1.81(\mathrm{~m}, 3 \mathrm{H}), 1.46-1.35(\mathrm{~m}, 2 \mathrm{H}), 1.04-0.98(\mathrm{~m}, 1 \mathrm{H}), 0.86(\mathrm{t}, J=7.4 \mathrm{~Hz}, 3 \mathrm{H})$.

\section{2) Western-blot of IDO1}

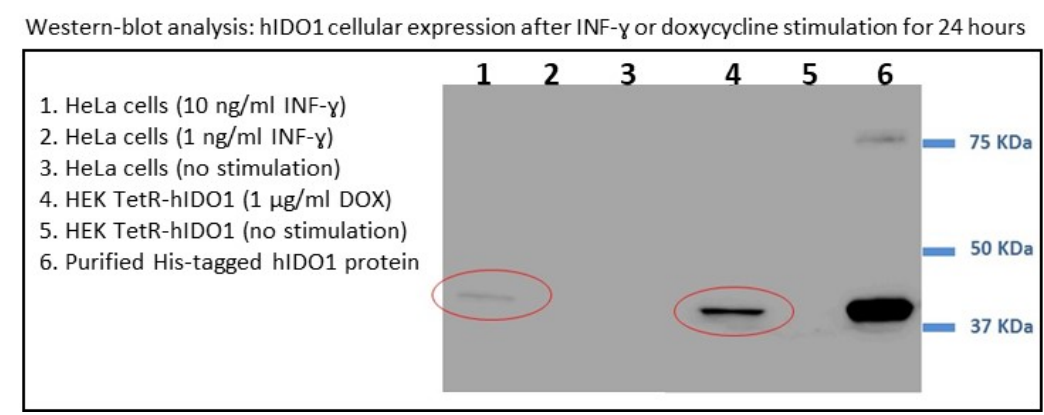

Figure S1. Western-Blot of IDO1 from cell lysates and purified His-tagged protein

\section{3) Microsomal stability assay}

Microsomal stability assay was conducted on a Beckmann Biomek FXp laboratory automation system. The liver microsomal incubation mixture consisted of liver microsomes $(0.5 \mathrm{mg}$ microsomal protein $/ \mathrm{ml})$, the test compound $(1 \mathrm{uM}), \operatorname{MgCl} 2(3 \mathrm{mM})$, EDTA $(1 \mathrm{mM})$ in potassium phosphate buffer (100 mM, pH 7.4). Midazolam and Ketanserin were used as the assay control substrate. The reaction was initiated with the addition of an NADPH regeneration solution (1.3 $\mathrm{mM}$ NADPH) and maintained at $37^{\circ} \mathrm{C}$ with shaking. At five time points ranging from 0 to $45 \mathrm{~min}$, 
aliquots $(50 \mathrm{uL})$ were removed and quenched with acetonitrile $(100 \mu \mathrm{L})$ containing an internal standard (imipramine). After vortex and centrifugation, samples were analyzed by LC-MS/MS. Calculation of the in vitro T1/2 and clearance followed literature. (Drug Metabolism and Disposition (1999), 27 (11): 1350-1359)

\section{4) CYP Inhibition Assays.}

Studies were carried out in human liver microsomes $(0.1 \mathrm{mg} / \mathrm{mL}$ for CYP $1 \mathrm{~A} 2 / 2 \mathrm{C} 9 / 2 \mathrm{D} 6 / 3 \mathrm{~A} 4 ; 0.5$ $\mathrm{mg} / \mathrm{mL}$ for CYP 2C19). Liver microsomes were purchased from BD Gentest. Aliquots of the compound stock solutions were diluted to $4 \mathrm{mM}$ with acetonitrile, and then further diluted upon the addition of liver microsomes $(0.2 \mathrm{mg} / \mathrm{mL})$. An aliquot of $30 \mu \mathrm{L}$ of diluted test compound solution was mixed with $15 \mu \mathrm{L}$ of substrate solution. The plate was pre-warmed to $37^{\circ} \mathrm{C}$, then 15 $\mu \mathrm{L}$ of $8 \mathrm{mM}$ NADPH (also pre-warmed to $37^{\circ} \mathrm{C}$ ) was added. The plate was incubated at $37^{\circ} \mathrm{C}$ for the following incubation times: $5 \mathrm{~min}$ for 3A4, $10 \mathrm{~min}$ for 1A2/2C9/2D6, and $45 \mathrm{~min}$ for $2 \mathrm{C} 19$. The reaction was stopped by adding acetonitrile at the designated time point. The assay plates were shaken on a vibrator (IKA, MTS 2/4) for $10 \mathrm{~min}(600 \mathrm{rpm})$ and centrifuged at 5,594 $\mathrm{g}$ for $15 \mathrm{~min}$ (Thermo Multifuge $\times 3 R$ ). Aliquots of the supernatant were taken, diluted 1:3 into distilled water, and metabolite concentrations were analyzed by LCMS/MS compared to internal standards. Substrates used were Phenacetin $(30 \mu \mathrm{M})$ for 1A2, Diclofenac $(10 \mu \mathrm{M})$ for 2C9, S-Mephenytoin $(35 \mu \mathrm{M})$ for $2 \mathrm{C} 19$, Midazolam $(10 \mu \mathrm{M})$ or Testosterone $(80 \mu \mathrm{M})$ for $3 \mathrm{~A} 4$, and Bufuralol $(10 \mu \mathrm{M})$ for 2D6. Metabolites measured were Acetaminophen for 1A2, 4'-Hydroxy-Diclofenac for 2C9, Hydroxy-Mephenytoin for 2C19, 1-Hydroxy-Midazolam or Osalmid for 3A4, and 1-HydroxyBufuralol for 2D6. Positive control inhibitors used were $\alpha$-Naphthoflavone for CYP1A2, Sulfaphenazole for CYP2C9, Omeprazole for CYP2C19, Quinidine for CYP2D6 and Ketoconazole for CYP3A4. The peak area response ratio (PARR) of metabolite to internal standard of the metabolites in test compound samples at desired time points was compared to the PARR in control samples to determine the percent of control sample ( $\%$ control $)$ at each time point. $\%$ inhibition was calculated as $100 \%$ control.

\section{5) Plasma protein binding assay}


Plasma protein binding (PPB) assay was conducted using the Rapid Equilibrium Dialysis (RED) device (ThermoFisher Scientific). Warfarin and metoprolol were used as the control substrate. To the Receiver side was added $350 \mu \mathrm{L}$ of phosphate buffered saline ( $\mathrm{pH} 7.4,1 \mathrm{x}$, Gibco). To the Donor side was added $200 \mu \mathrm{L}$ of plasma (Bioreclamation IVT) spiked with the test compound (5 $\mu \mathrm{M})$. The same plasma/the test compound solution $(50 \mu \mathrm{L})$ was also used for the Recovery sample. The plate was covered with Immunoware sealing tape and was incubated at $37^{\circ} \mathrm{C}$ with shaking at $100 \mathrm{rpm}$ for $5 \mathrm{hr}$. After the incubation, both the receiver and donor sides were sampled (50 $\mu \mathrm{L})$ and matched with the same volume of matrix from the other side. The Recovery, Donor, and Receiver samples were extracted with $300 \mu \mathrm{L}$ of cold acetonitrile containing imipramine as the internal standard. After vortex and centrifugation, the supernatant $(150 \mu \mathrm{L})$ was subjected to LCMS quantitation. PPB (\% bound) was calculated as \%Bound = 100x ([Donor]-[Receiver]/[Donor].

\section{6) hERG Fluorescence Polarization Assay.}

hERG binding was assessed by incubating compounds with Predictor hERG membrane (ThermoFisher K1785) and using Tracer Red, a high affinity red fluorescent hERG channel ligand, as the substrate (ThermoFisher PV5363). In a 384 well black plate (Greiner 784900), predictor hERG membranes $(2 \mathrm{X})$ were incubated with or without increasing concentrations of inhibitor compounds in assay buffer ( $25 \mathrm{mM}$ HEPES, $15 \mathrm{mM} \mathrm{KCl}, 1 \mathrm{mM} \mathrm{MgCl} 2,0.5 \mu \mathrm{M}$ pluronic F-127), E-4031 $(10 \mu \mathrm{M})$ as the control compound, and the substrate hERG Tracer Red $(250 \mathrm{nM})$ at room temperature with a final reaction volume of $20 \mu \mathrm{L}$. After 3 hours, fluorescence polarization was measured (Excitation: 530/25, Emission: 590/35) using a Synergy Neo (Biotek). Gain was automatically set using a background buffer well on the plate containing $2 \mathrm{X}$ hERG membranes and $10 \mathrm{uL}$ of assay buffer. Dose-response curves were analyzed using IC50 regression curve fitting (GeneData Screener).

\section{7) QPatch (Automated Electrophysiology) Assay}

The assay was performed at ChemPartner (No. 5 Building, 998 Halei Road, Zhangjiang HiTech Park, Pudong New Area, Shanghai, China. www.chempartner.com). Cell culture and cell requirements. A CHO cell line stably transfected with hERG cDNA and expressing hERG 
channels of P24 was used for the study. Cells were maintained in petri dishes or flasks at $37{ }^{\circ} \mathrm{C}$ in a humidified incubator with 5\% $\mathrm{CO}_{2}$ and cultured in medium (from Invitrogen) containing: Ham's F12, $10 \%$ (v/v) heat inactivated FBS, $100 \mu \mathrm{g} / \mathrm{ml}$ Hygromycin B, $100 \mu \mathrm{g} / \mathrm{ml}$ Geneticin. Cells were allowed to grow and reach a confluence rate at about $80-90 \%$ in the above condition. Before the experiment, the cells were treated with Detachin (from Genlantis) for 3-5 min. at 37 ${ }^{\circ} \mathrm{C}$ followed by gentle pipette tituration 15-20 times at room temperature with culture media, then the cells were re-suspended in serum-free culture medium containing CHO-S-SFM II medium(from Invitrogen) buffered with HEPES (25 mM). The cells used in QPatch study must meet following criteria: under microscopy examination, the majority of cells in suspension should be single and isolated; their viability should be greater than $95 \%$, with only a few debris and cell clumps (which may clog the holes in QPlate during whole-cell clamp recording); cell density should be ranged within $3-8 \times 10^{6}$ cells $/ \mathrm{ml}$ in the final suspension before applying to the QPatch stir chamber. Cells in such condition can be used for recording only for four hours after harvesting.

For the electrophysiological recordings the following solutions (recommended by Sophion) were used.

Table S1. Composition of internal and external solutions used in hERG studies

\begin{tabular}{|c|c|c|}
\hline Reagent & External Solution (mM) & Internal Solution (mM) \\
\hline $\mathrm{CaCl}_{2}$ & 2 & 5.374 \\
\hline $\mathrm{MgCl}_{2}$ & 1 & 1.75 \\
\hline $\mathrm{KCl}$ & 4 & 120 \\
\hline $\mathrm{NaCl}$ & 145 & - \\
\hline Glucose & 10 & - \\
\hline HEPES & 10 & 10 \\
\hline EGTA & - & 5 \\
\hline Na-ATP & - & 4 \\
\hline $\begin{array}{r}\mathrm{pH} \& \\
\text { Osmolarity }\end{array}$ & $\begin{array}{l}7.4 \text { (adjusted with } \mathrm{NaOH} \text { ), } \\
\text { osmolarity } \sim 305 \text { mOsm }\end{array}$ & $\begin{array}{c}7.25 \text { (adjusted with } \mathrm{KOH} \text { ), } \\
\text { osmolarity } \sim 295 \text { mOsm }\end{array}$ \\
\hline
\end{tabular}


Table S2. Chemicals and reagents information

\begin{tabular}{|l|l|l|l|l|}
\hline Chemicals and reagents & \multicolumn{1}{|c|}{ Catalog No. } & \multicolumn{1}{|c|}{ Lot No. } & \multicolumn{1}{c|}{ SW } & Supplier \\
\hline $\mathrm{NaCl}$ & S1679-1KG & WXBC1368V & 58.44 & Sigma \\
\hline $\mathrm{KCl}$ & $31248-100 \mathrm{G}$ & WXBC2571V & 74.55 & Sigma \\
\hline $\mathrm{CaCl} 2$ (1M solution) & $21114-1 \mathrm{~L}$ & BCBM6063V & 110.98 & Sigma \\
\hline $\mathrm{MgCl} 2 \cdot 6 \mathrm{H} 2 \mathrm{O}$ & M7304-100G & V900020-500G & 203.30 & Sigma \\
\hline HEPES & H3375-1KG & SLBP2246V & 238.30 & Sigma \\
\hline Glucose & G8270-1KG & WXBC2393V & 180.16 & Sigma \\
\hline EGTA & 03777-50G & SLBP2807V & 380.15 & Sigma \\
\hline Na2-ATP & A-7699-5G & SLBJ8915V & 551.14 & Sigma \\
\hline NaOH (2M solution) & $35254-1 \mathrm{~L}$ & BCBG6297V & 40.00 & Sigma \\
\hline KOH & 232041-50G & SLBK9251V & 149.91 & Sigma \\
\hline DMSO & D276855-1L & STBF-7845V & 78.13 & Sigma \\
\hline
\end{tabular}

Recording system. Whole-cell recordings were performed using automated QPatch (Sophion Biosciences, Denmark). The cells were voltage clamped at a holding potential of $-80 \mathrm{mV}$. The hERG current was activated by depolarizing at $+20 \mathrm{mV}$ for $5 \mathrm{sec}$, after which the current was taken back to $-50 \mathrm{mV}$ for $2.5 \mathrm{sec}$ to remove the inactivation and observe the outward tail current. The maximum amount of tail current was used to determine hERG current amplitude.

Compound handling and dilutions. Compounds were received from client as 10mM DMSO stock on Jun. 05,2017 . Six doses $(30,10,3,1,0.3$ and $0.1 \mu \mathrm{M})$ were chosen to obtain fitting curves and $\mathrm{IC}_{50}$. Before QPatch test, compounds were serial-diluted in external solution to the final $\mu \mathrm{M}$ ranges. 5-10 min. of sonication and vortex were routinely applied to the solutions to assist the complete dissolution of compound before the experiments. Final DMSO concentration was $0.1 \%$ or less except for $30 \mu \mathrm{M}$ which was about $0.3 \%$. Doses for $\mathrm{IC}_{50}$ evaluation of positive control cisapride were $3,1,0.3,0.1,0.03$, and $0.01 \mu \mathrm{M}$.

Automated QPatch procedures. After achieving break-in (whole-cell) configuration, the cells were recorded for $120 \mathrm{sec}$ to assess current stability. The voltage protocol described above was then applied to the cells every $15 \mathrm{sec}$ throughout the whole procedure. Only stable cells with recording parameters above threshold were allowed to enter the drug application procedure. All experiments were conducted at room temperature (about $25^{\circ} \mathrm{C}$ ). Each measurements were in duplicate at each dose. External solution containing $0.1 \%$ DMSO (vehicle) was applied to the cells 
to establish the baseline. After allowing the current to stabilize for 3 minutes, compound was applied. Compound solution was added and the cells were kept in the test solution until the compound's effect reached a steady state or for a maximum of $4 \mathrm{~min}$. For dose response assay, compound was applied to the cells accumulatively from low to high concentrations. Washout with external solution was performed after compound testing. Positive control cisapride is used in the experiments to test the same batch of cells used for test compounds to ensure the normal response and the good quality of the cells.

Data Analysis. Data were analyzed using Assay Software provided by Sophion(assay software V5.0), Microsoft Excel and Graphpad Prism 5.0.

Quality Control. Data included in the report originated from experiments which satisfied all of the following criteria. Recording parameters: membrane resistance $R_{m}>300 M \Omega$, access resistance $\left(\mathrm{R}_{\mathrm{a}}\right)<15 \mathrm{M} \Omega$, tail current amplitude $>200 \mathrm{pA}$, rundown $<2 \%$ per minute, leak current $<200 \mathrm{pA}$ or smaller than $10 \%$ of the peak tail current size in $90 \%$ of the recording time. Pharmacological parameters: Cisapride (C4740-10mg, Sigma) in multiple doses blocked hERG current as a positive control.

\section{8) Manual Patch Clamp}

The assay was performed at ChemPartner (No. 5 Building, 998 Halei Road, Zhangjiang HiTech Park, Pudong New Area, Shanghai, China. www.chempartner.com). Cell culture and cell preparation for manual patch. A CHO cell line stably transfected with hERG cDNA and expressing hERG channels of P9 were used for the study. Cells were cultured in medium (from Invitrogen) containing: Ham's F12, 10 \% (v/v) heat inactivated FBS, $100 \mu \mathrm{g} / \mathrm{ml}$ Hygromycin B, and $100 \mu \mathrm{g} / \mathrm{ml}$ Geneticin. Flasks or dishes of CHO hERG cells were maintained in the above medium and incubated at $37^{\circ} \mathrm{C}$ in a humidified incubator with $5 \% \mathrm{CO}_{2}$ for 24 to 48 hours prior to electrophysiological recordings, the cells were plated on glass cover slips placed in culture dishes and maintained under the same incubation and media conditions. The number of cells being plated on the cover slip should reach a confluence rate at which majority of the cells are single. For the electrophysiological recordings the following solutions were used.

Table S3. Composition of internal and external solutions used in hERG patch clamp studies 


\begin{tabular}{|c|c|c|}
\hline Reagent & External Solution (mM) & Internal Solution $(\mathrm{mM})$ \\
\hline $\mathrm{CaCl}_{2}$ & 2 & 5.374 \\
\hline $\mathrm{MgCl}_{2}$ & 1 & 1.75 \\
\hline $\mathrm{KCl}$ & 4 & 120 \\
\hline $\mathrm{NaCl}$ & 145 & - \\
\hline Glucose & 10 & - \\
\hline HEPES & 10 & 10 \\
\hline EGTA & - & 5 \\
\hline Na-ATP & - & 4 \\
\hline $\begin{array}{r}\mathrm{pH} \\
\text { \&Osmolarity }\end{array}$ & $\begin{array}{l}7.4 \text { (adjusted with } \mathrm{NaOH} \text { ), } \\
\text { Osmolarity } \sim 305 \text { mOsm }\end{array}$ & $\begin{array}{l}7.25 \text { (adjusted with } \mathrm{KOH} \text { ), } \\
\text { Osmolarity } \sim 280 \mathrm{mOsm}\end{array}$ \\
\hline
\end{tabular}

Table S4. Chemicals and reagents information

\begin{tabular}{|c|c|c|c|c|}
\hline $\begin{array}{c}\text { Chemicals and } \\
\text { reagents }\end{array}$ & Catalog No. & Lot No. & MW & Supplier \\
\hline $\mathrm{NaCl}$ & S1679-1KG & WXBC1368V & 58.44 & Sigma \\
\hline $\mathrm{KCl}$ & $31248-100 \mathrm{G}$ & WXBC2571V & 74.55 & Sigma \\
\hline $\mathrm{CaCl} 2(1 \mathrm{M}$ solution) & $21114-1 \mathrm{~L}$ & BCBM6063V & 110.98 & Sigma \\
\hline $\mathrm{MgCl} \cdot 6 \mathrm{H} 2 \mathrm{O}$ & $\mathrm{M} 7304-100 \mathrm{G}$ & $\begin{array}{l}\text { V900020- } \\
500 \mathrm{G}\end{array}$ & 203.30 & Sigma \\
\hline $\mathrm{HEPES}$ & $\mathrm{H} 3375-1 \mathrm{KG}$ & SLBP2246V & 238.30 & Sigma \\
\hline Glucose & G8270-1KG & WXBC2393V & 180.16 & Sigma \\
\hline $\mathrm{EGTA}$ & $03777-50 \mathrm{G}$ & SLBP2807V & 380.15 & Sigma \\
\hline $\mathrm{Na} 2-\mathrm{ATP}$ & $\mathrm{A}-7699-5 \mathrm{G}$ & SLBJ8915V & 551.14 & Sigma \\
\hline $\mathrm{NaOH}(2 \mathrm{M}$ solution) & $35254-1 \mathrm{~L}$ & BCBG6297V & 40.00 & Sigma \\
\hline $\mathrm{KOH}$ & $232041-50 \mathrm{G}$ & SLBK9251V & 149.91 & Sigma \\
\hline $\mathrm{DMSO}$ & $\mathrm{D} 2650-100 \mathrm{ML}$ & STBF-7845V & 78.13 & Sigma \\
\hline
\end{tabular}

Manual whole cell patch and recording procedures. HEKA EPC 10 USB patch clamp amplifier (from HEKA Elektronik, Germany) was used in the whole cell recording. A cover slip with plenty of single CHO hERG cells on the surface was removed and placed into a continuously perfused (approximately 1 $\mathrm{ml} /$ minute) recording chamber mounted on an inverted microscope. HERG channel currents were recorded from single cells using standard whole cell recording techniques. The cells were voltage clamped at a holding potential of $-80 \mathrm{mV}$. The hERG current was activated by depolarizing at $+20 \mathrm{mV}$ for $5 \mathrm{sec}$, after which the current was taken back to $-50 \mathrm{mV}$ for $5 \mathrm{sec}$ to remove the inactivation and observe 
the deactivating tail current. The $\mathrm{K}^{+}$tail current through HERG channels observed during this step was allowed to stabilize under continuous bath perfusion. Cells were then superfused with drug until steady state block was achieved. Steady state was considered reached when three consecutive super-imposable current records were collected. At this point, cells were once again superfused with extracellular solution until the current amplitude returned to values close to those measured before application of drug. One or more compounds or concentrations of drugs were tested on each cell with washout in between each drug application. Cisapride was used in the experiments to ensure the normal response and good quality of the hERG cells.

Compound handling and dilutions. Compounds were received from client as $10 \mathrm{mM}$ DMSO stock. Six doses $(30,10,3,1,0.3,0.1 \mu \mathrm{M})$ were chosen to obtain fitting curves and $\mathrm{IC}_{50}$ for these compounds. Before experiments, compounds were first made into 3, 1, 0.3, 0.1 mM DMSO stock, then further diluted in external solution by serial dilutions into the final desired concentrations. Final DMSO concentration was below 1\%. 5-10 min. of sonication and vortex were routinely applied to the solutions to assist the complete dissolution of compound.

Data Analysis. Data were analyzed using Assay Software provided by Patchmaster and Graphpad Prism 5.0.

Quality Control. Data included in the report originated from experiments which satisfied all of the following criteria. Recording parameters: membrane resistance $R_{m}>500 \mathrm{M} \Omega$, access resistance $\left(\mathrm{R}_{\mathrm{a}}\right)<10 \mathrm{M} \Omega$, tail current amplitude $>200 \mathrm{pA}$, rundown $<2 \%$ per minute, leak current $<200 \mathrm{pA}$ or $10 \%$ of the tail current amplitude in $90 \%$ of the recording time. Pharmacological parameters: Cisapride (C4740-10mg, Sigma) in multiple doses blocked hERG current as a positive control.

\section{9) X-ray Crystallography}

Crystals of human IDO1 in complex with compound 22 were grown using hanging drop vapor diffusion method at $293 \mathrm{~K}$ by mixing protein solution ( $25 \mathrm{mg} / \mathrm{mL}$ IDO1 (high-heme) in $25 \mathrm{MES}$ $\mathrm{pH} 6.5,150 \mathrm{mM} \mathrm{KCl}$ preincubated with $20 \mathrm{mM}$ ligand) with reservoir solution (100 $\mathrm{mM}$ Tris $\mathrm{pH}$ $8.0,18 \%(\mathrm{w} / \mathrm{v})$ PEG $6000,0.2 \mathrm{M} \mathrm{NaCl})$ in a 1:1 ratio. To obtain high resolution diffraction data of human IDO1 in complex with 10, crystals were optimized and flash cooled using the free mounting system ${ }^{1}$. Diffraction data were collected at $100 \mathrm{~K}$ at the Swiss Light Source (SLS, Villigen Switzerland) and processed using XDS and XSCALE ${ }^{2}$. Structures were solved using 
Phaser $^{3}$ and a previously solved hIDO structure as search model. Subsequent model building and refinement was performed with $\mathrm{COOT}^{4}$ and REFMAC 5 .

For the structures of human IDO1 with compounds $\mathbf{1 7}$ and $\mathbf{7 0}$ the crystals were prepared as described for the co-crystal with compound 22 except that they were cryo-protected by plunge freezing in liquid nitrogen after briefly soaking the crystals in cryo-protectant $(100 \mathrm{mM}$ Tris $\mathrm{pH}$ 8.0, 20\% PEG6000, 0.2 M NaCl and 10\% PEG 400). Diffraction data was collected at the 22-BM beamline (Advanced Photon Source, Lemont, USA) and processed using iMOSFLM ${ }^{6}$. The structures were solved by molecular replacement using PDB 5ETW as the search model, and iteratively refined using $\mathrm{PHENIX}^{7}$ and $\mathrm{COOT}^{4}$.

Table S5. Data collection and refinement statistics.

\begin{tabular}{|c|c|c|c|}
\hline & IDO1:Compound 22 & IDO1:Compound 70 & IDO1:Compound 17 \\
\hline PDB Entry & $7 \mathrm{~B} 1 \mathrm{O}$ & $7 \mathrm{M} 63$ & 7M7D \\
\hline Wavelength $(\AA)$ & 1.00000 & 1.00000 & 1.00000 \\
\hline Resolution range $(\AA)$ & $45.76-2.58(2.83-2.58)^{*}$ & $46.03-3.1(3.211-3.1)^{*}$ & $33.29-2.6(2.693-2.6)^{*}$ \\
\hline Space group & $\mathrm{P} 2{ }_{1} 2_{1} 2_{1}$ & $\mathrm{P} 22_{1} 2_{1} 2_{1}$ & $\mathrm{P} 2{ }_{1} 2_{1} 2_{1}$ \\
\hline $\begin{array}{l}\text { Unit cell }(\AA) \\
\quad\left({ }^{\circ}\right)\end{array}$ & $\begin{array}{c}84.5691 .526130 .279 \\
909090\end{array}$ & $\begin{array}{c}84.9192 .07128 .29 \\
909090\end{array}$ & $\begin{array}{c}85.8691 .80128 .78 \\
909090\end{array}$ \\
\hline Unique reflections & $32082(7432)^{*}$ & $18622(1829)^{*}$ & $31967(3140)^{*}$ \\
\hline Multiplicity & $5.0(4.9)^{*}$ & $34.9(33.9)^{*}$ & $47.3(47.6)^{*}$ \\
\hline Completeness $(\%)$ & $98.64(95.8)^{*}$ & $97.54(97.90)^{*}$ & $99.89(99.97)^{*}$ \\
\hline Mean I/sigma(I) & $18.66(4.18)^{*}$ & $18.99(1.56)^{*}$ & $120.90(3.41)^{*}$ \\
\hline Wilson B-factor & 64.24 & 70.60 & 69.15 \\
\hline R-merge & $0.510(0.421)^{*}$ & $1.137(5.873)^{*}$ & $0.7351(3.289)^{*}$ \\
\hline R-meas & $0.570(0.471)^{*}$ & $1.154(5.965)^{*}$ & $0.7431(3.325)^{*}$ \\
\hline R-pim & & $0.196(1.03)^{*}$ & $0.1076(0.4853)^{*}$ \\
\hline $\mathrm{CC} 1 / 2$ & & $0.865(0.201)^{*}$ & $0.914(0.427)^{*}$ \\
\hline $\mathrm{CC}^{*}$ & & $0.963(0.579)^{*}$ & $0.977(0.774)^{*}$ \\
\hline $\begin{array}{l}\text { Reflections used in } \\
\text { refinement }\end{array}$ & 32076 & 18393 & 31953 \\
\hline Reflections used for R-free & 857 & 917 & 1609 \\
\hline R-work & 0.2540 & 0.2510 & 0.2087 \\
\hline R-free & 0.2962 & 0.2848 & 0.2353 \\
\hline $\begin{array}{l}\text { Number of non-hydrogen } \\
\text { atoms }\end{array}$ & 6064 & 5924 & 6066 \\
\hline macromolecules & 5965 & 5866 & 5919 \\
\hline ligands & 60 & 58 & 140 \\
\hline solvent & 39 & 0 & 7 \\
\hline Protein residues & 750 & 739 & 745 \\
\hline RMS(bonds) & 0.010 & 0.002 & 0.002 \\
\hline RMS(angles) & 1.17 & 0.51 & 0.54 \\
\hline Ramachandran favored (\%) & 97.71 & 95.62 & 95.52 \\
\hline Ramachandran allowed (\%) & 2.29 & 4.38 & 4.48 \\
\hline Ramachandran outliers (\%) & 0.00 & 0.00 & 0.00 \\
\hline Rotamer outliers (\%) & 0.15 & 0.00 & 3.55 \\
\hline Clashscore & 0.25 & 6.17 & 5.16 \\
\hline Average B-factor & 40.79 & 67.71 & 85.15 \\
\hline
\end{tabular}




\begin{tabular}{|l|c|c|c|}
\hline macromolecules & 40.61 & 67.80 & 85.82 \\
\hline ligands & 49.01 & 58.20 & 57.92 \\
\hline Solvent & 55.99 & 0 & 61.20 \\
\hline Number of TLS groups & 6 & N/A & 8 \\
\hline
\end{tabular}

*Statistics for the highest-resolution shell are shown in parentheses.

\section{0) Sequence Alignment of the Binding Site of IDO1}

Table S6. Homology of IDO1 binding site across species

\begin{tabular}{|l|c|c|c|c|c|c|c|c|c|c|c|c|c|c|c|c|c|c|c|c|c|c|c|c|c|c|c|c|c|}
\hline hIDO1 AA & 126 & 129 & 130 & 163 & 164 & 166 & 167 & 170 & 171 & 174 & 207 & 214 & 217 & 221 & 234 & 262 & 263 & 264 & 267 & 269 & 270 & 273 & 274 & 339 & 342 & 343 & 346 & 349 & 388 \\
\hline human & Y & C & V & F & F & V & S & V & E & A & L & F & I & V & L & G & S & A & S & V & F & F & D & L & L & R & H & I & L \\
\hline mouse & Y & C & V & F & F & V & S & V & E & A & L & F & M & V & L & G & S & A & S & I & F & L & D & L & V & R & H & I & L \\
\hline rat & Y & C & V & F & F & V & S & V & E & A & L & F & M & V & L & G & S & A & S & I & F & L & D & L & L & R & H & I & L \\
\hline dog & Y & C & V & F & F & V & S & V & E & A & L & F & V & V & L & G & S & A & S & I & F & F & D & M & L & R & H & I & L \\
\hline Rhesus Macaque & Y & C & V & F & F & V & S & V & E & A & L & F & M & V & L & G & S & A & S & I & F & F & D & L & L & R & H & I & L \\
\hline
\end{tabular}

\section{1) Typical HeLa IC50 and viability (toxicity) curves.}

Typical $\mathrm{IC}_{50}$ and toxicity curves. None of the inhibitors showed any toxicity up to $10 \mu \mathrm{M}$ in our cell viability assay. Curves for compound 22 and 62 (IACS-9779, done in duplicate) are shown 


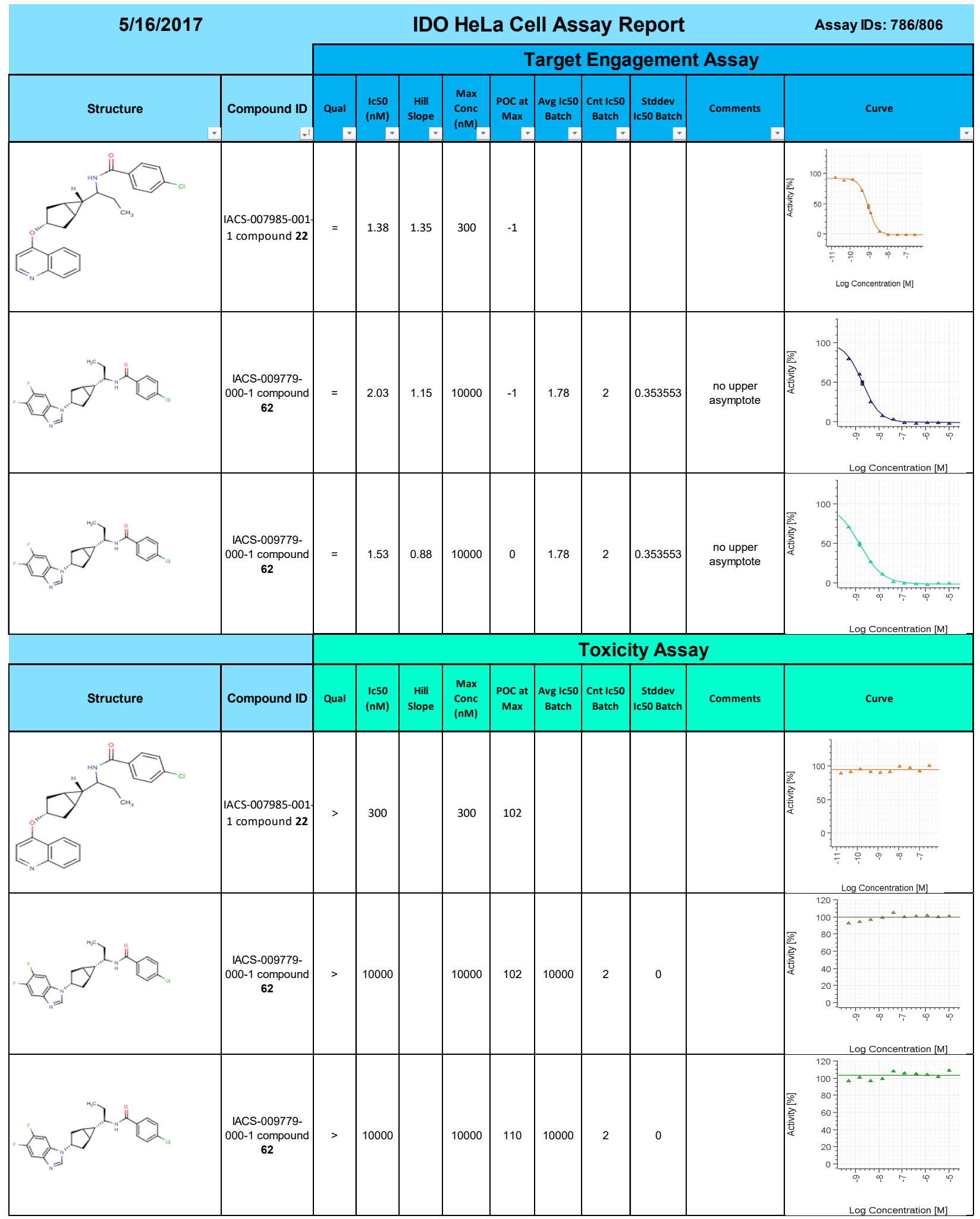

12) LCMS Traces of 17, 17R, 21, 22, 24, 31, 34, 38, 56, 57, 58, 59, 62 (IACS-9779), 63, 64, 65, 70 (IACS-70099), 71 (IACS-70465), and 72 
Openlynx Report - AP

Sample: 1

File:AP-0242-32-RYCRY-RL

Description:
Method:C:IMassLynxIStandard Run.olp Date:22-Feb-2017
Page 1

Vial:2:40

Time:11:09:10
1: MS ES+ :TIC Smooth (SG, $2 \times 2)$

(1)

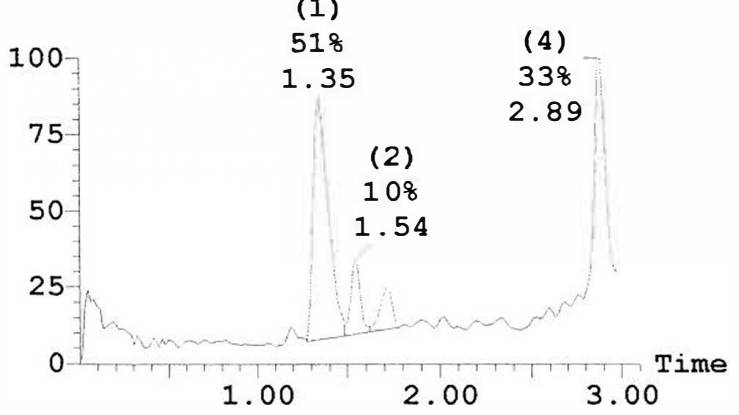

3: UV Detector: TIC

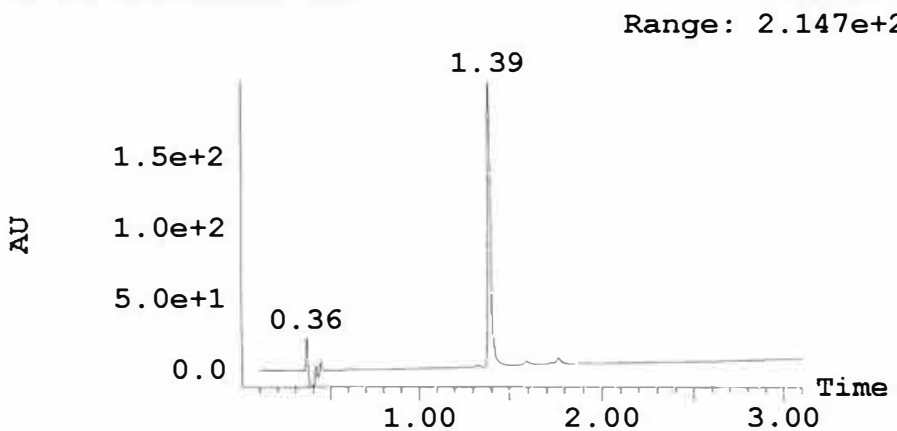

3. 0e+008 2: MS ES- :TIC Smooth (SG, 2x2)

3. $9 e+007$

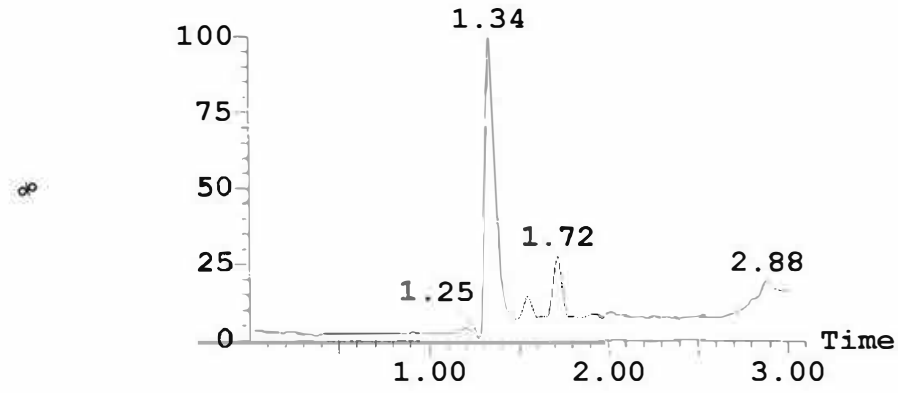

(1) ELSD Signal

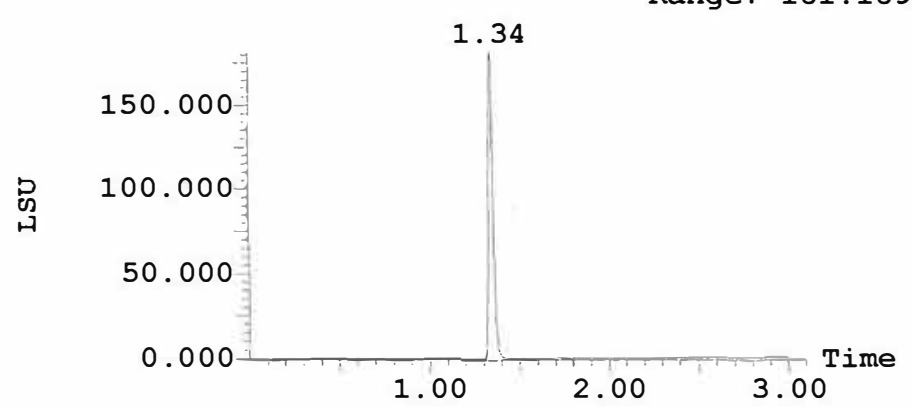

Peak ID Compound Time Mass Found

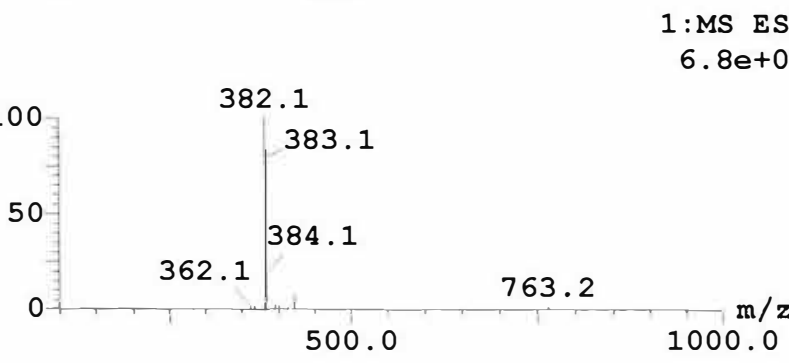

Peak ID Compound Time Mass Found 1.71

$1:$ MS ES+ $6.5 e+006$

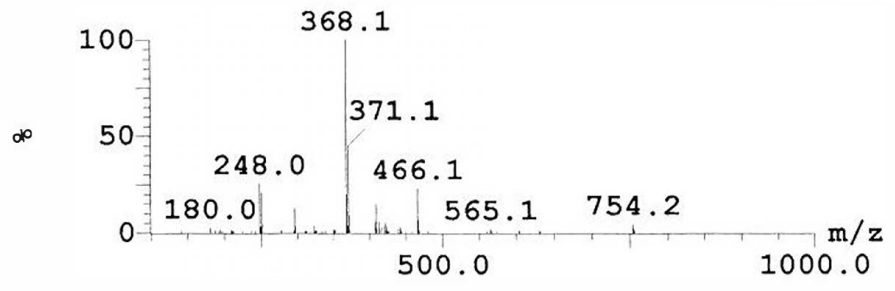

\section{Peak ID Compound Time Mass Found}

1 :MS ES+

1. $9 e+007$

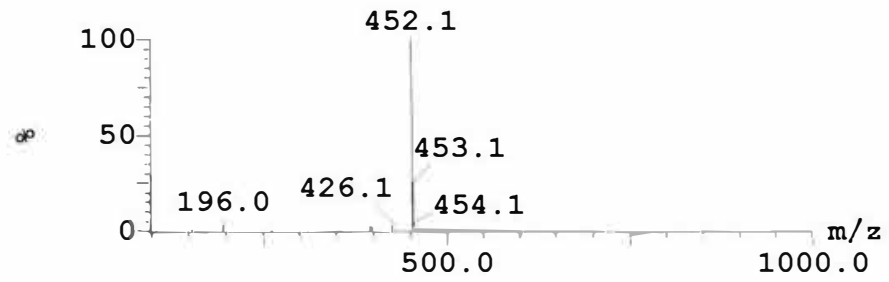

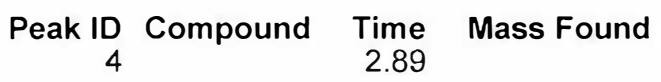

1:MS ES+ 2. $5 e+007$

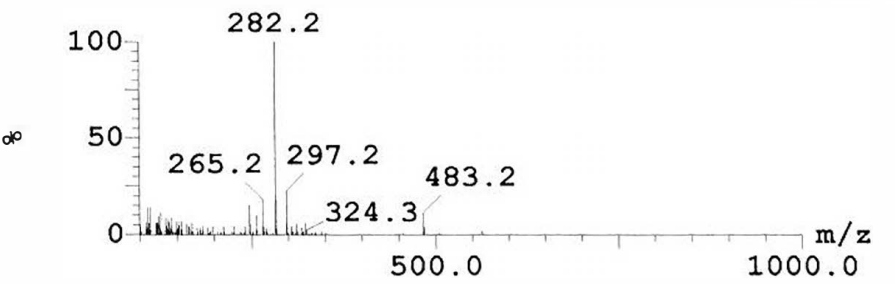


Vial: $1: 20$

Time:16:29:17
1: MS ES+ :TIC Smooth (SG, 2x2)

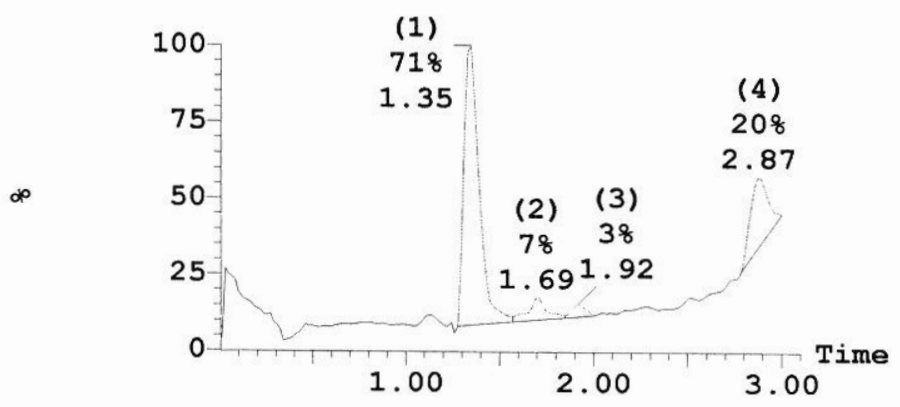

3: UV Detector: TIC

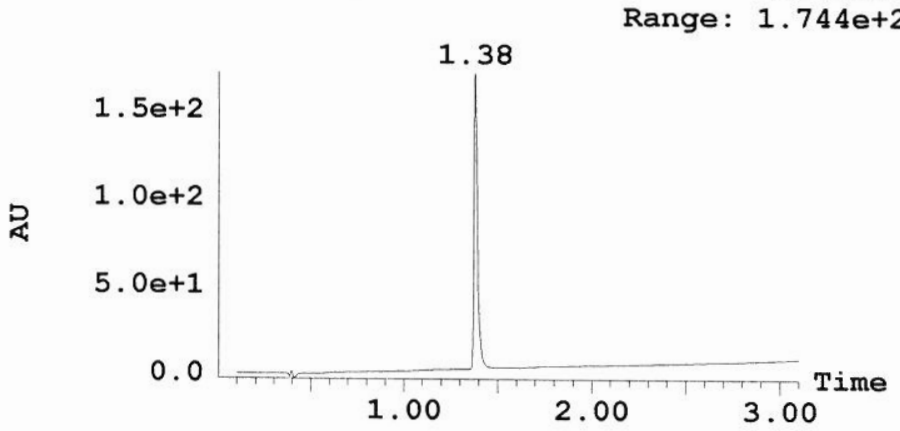

2.6e+008 2: MS ES- :TIC Smooth (SG, 2x2)

3. $3 e+007$

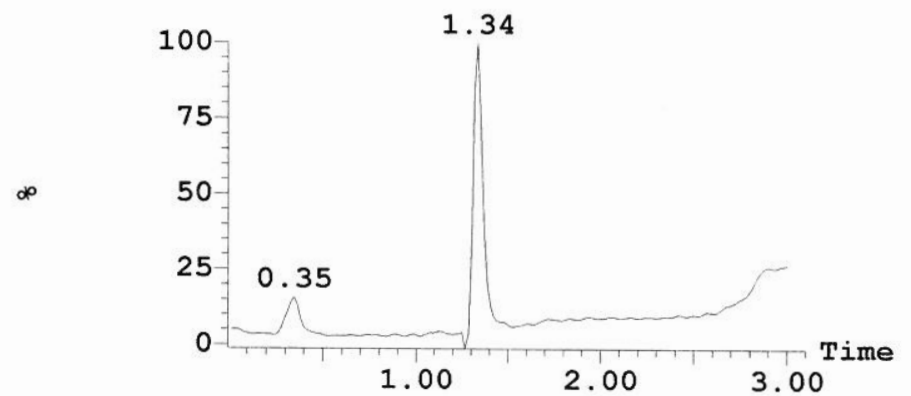

(1) ELSD Signal

144.734

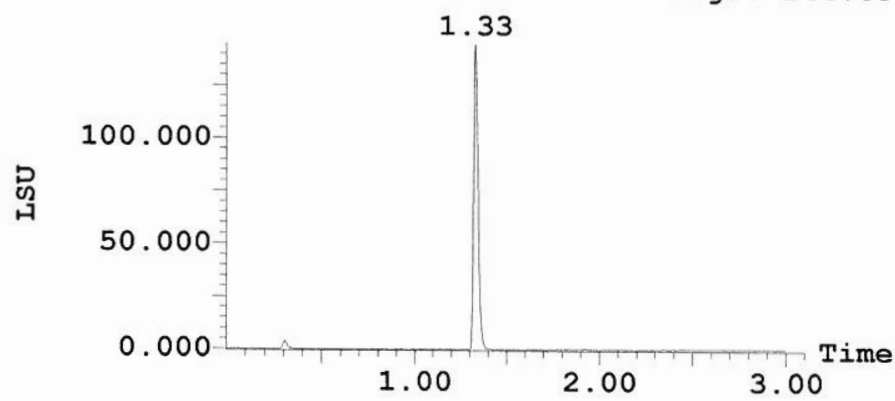

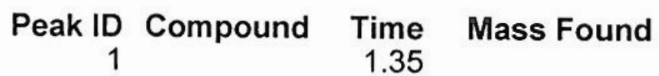
$1:$ MS ES+

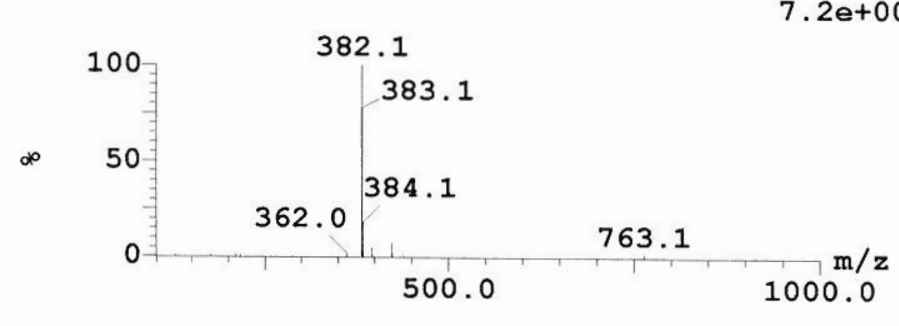

Peak ID Compound Time Mass Found
3

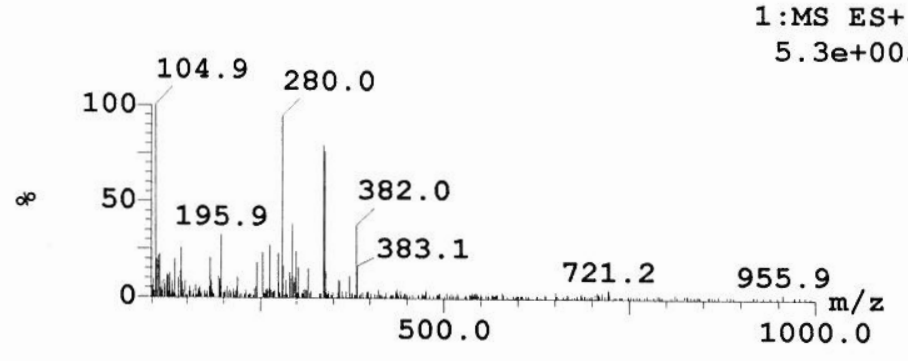

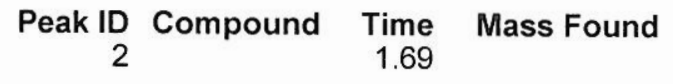

1 :MS ES+ $3.5 e+006$

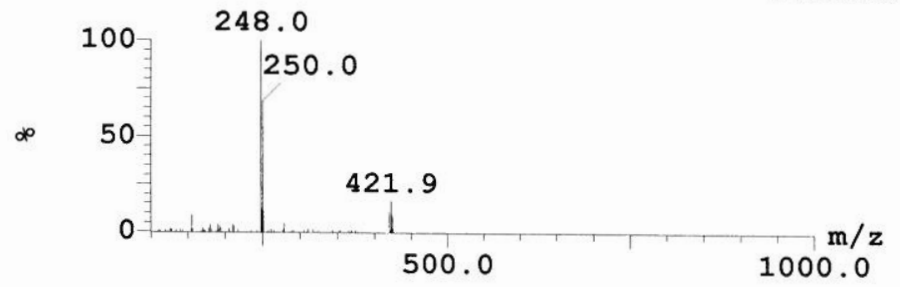

Peak ID Compound $\begin{array}{r}\text { Time } \\ 2.87\end{array} \quad$ Mass Found

1 :MS ES+

8. $7 e+006$

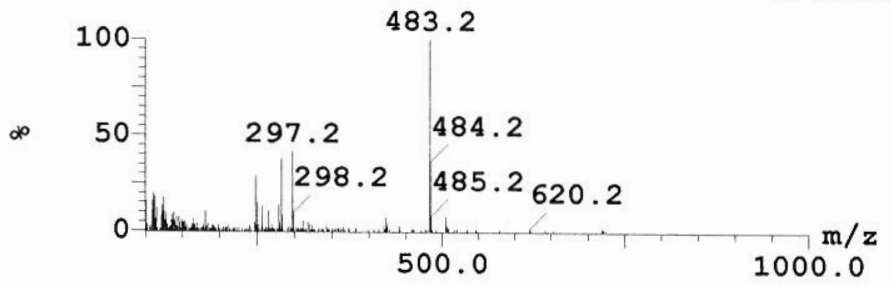


Openlynx Report - MJS

Sample: 1

File:MS-0190-46aMS

Description:
Method:C:MassLynxiStandard Run.olp Date:12-Jul-2016
Vial: $2: 8$

Time:15:49:06
1: MS ES+ : TIC Smooth (SG, 2×2)

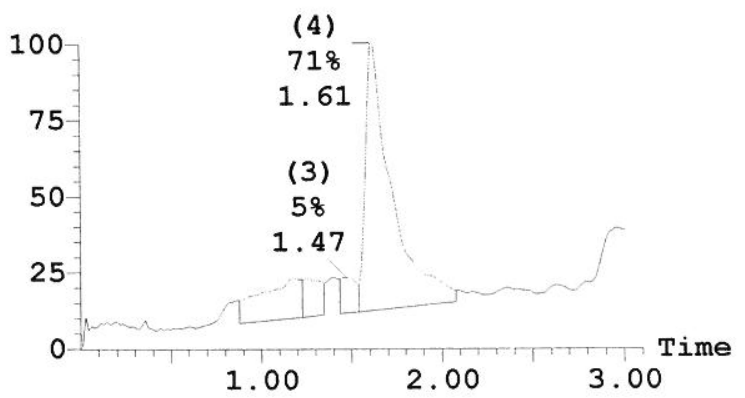

3: UV Detector: TIC

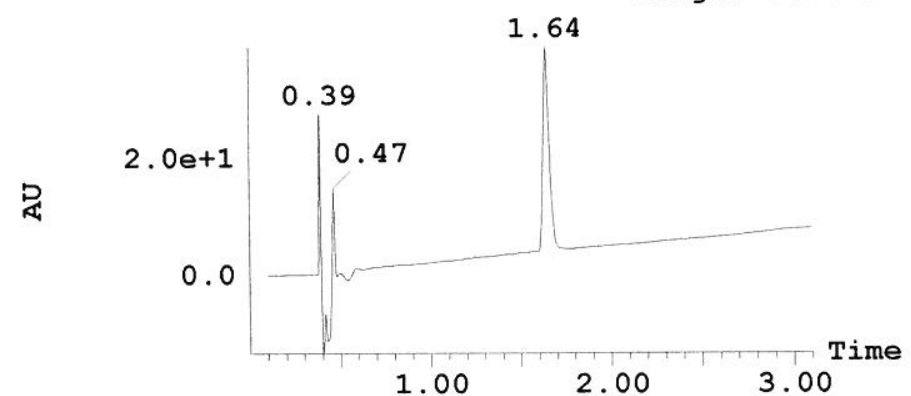

Peak ID Compound Time Mass Found 1.17

3. $927 e+1$ $+1$

(2) ELSD Signal

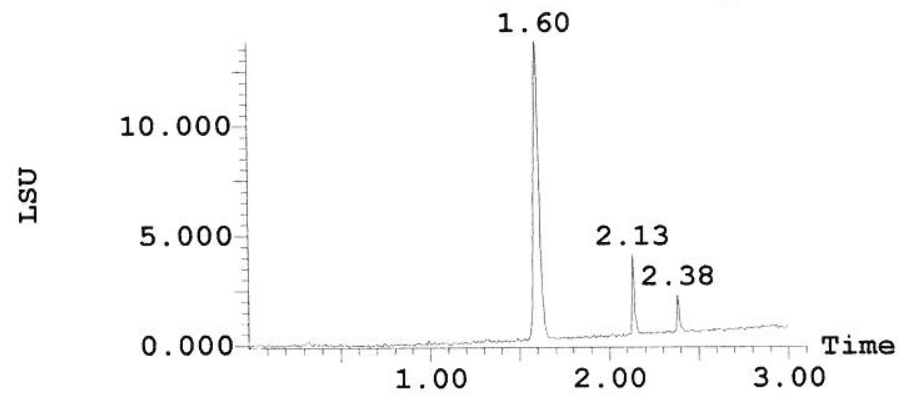

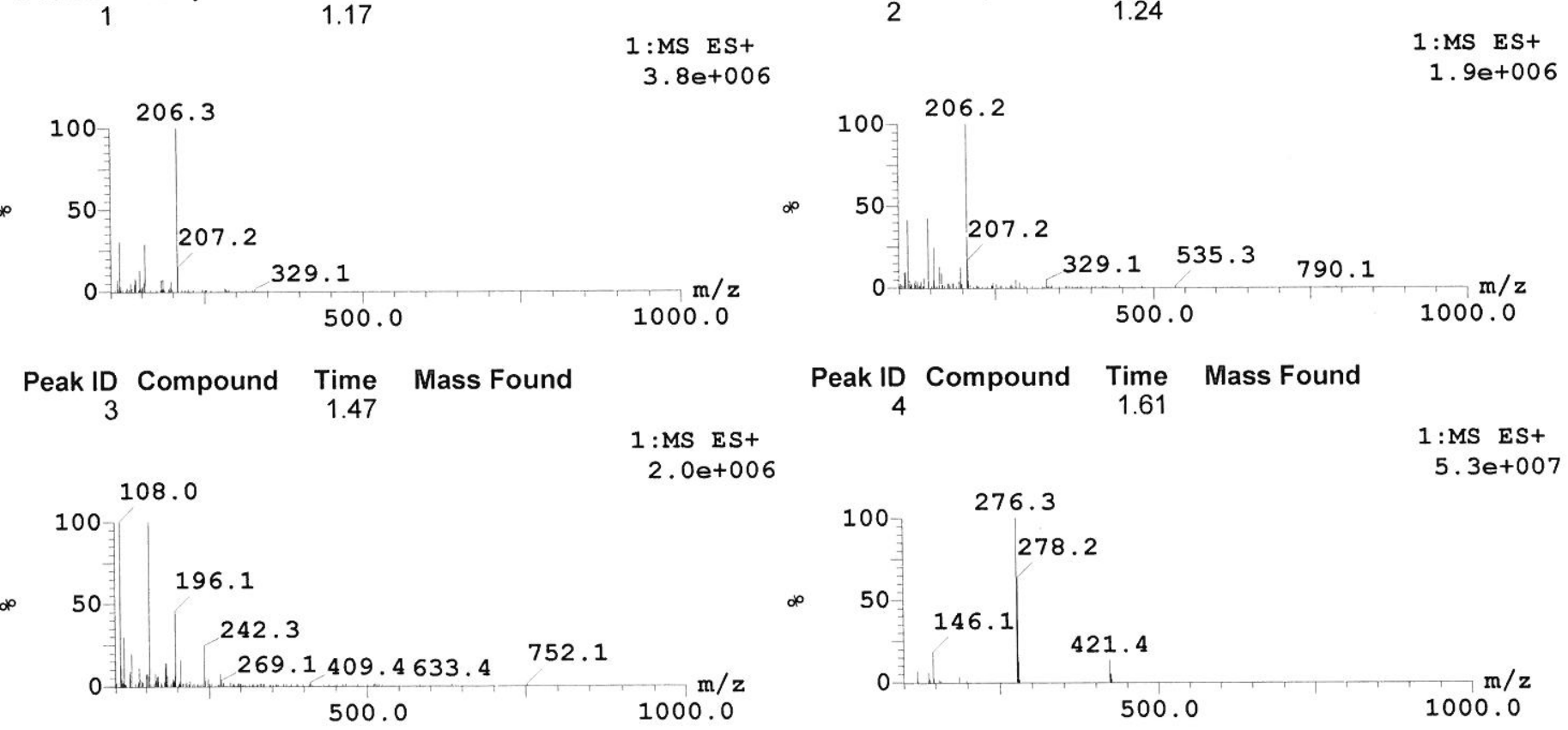


Openlynx Report - MJS

Sample: 1

File:MS-0190-47aMS

Description:
Method:C:MassLynxIStandard Run.olp Date:12-Jul-2016
Page 1

Vial:2:17

Time:16:31:25
1: MS ES+ :TIC Smooth (SG, 2×2)

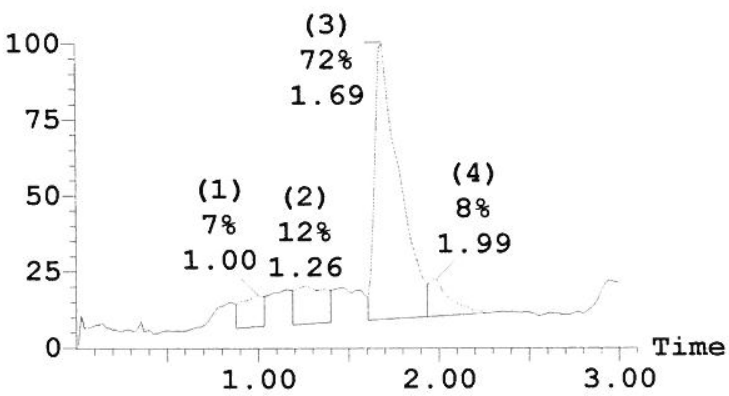

3: UV Detector: TIC

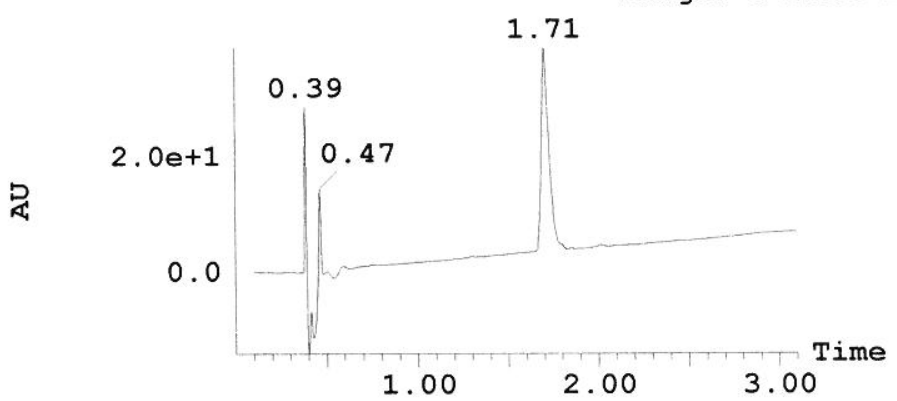

Peak ID Compound Time
1

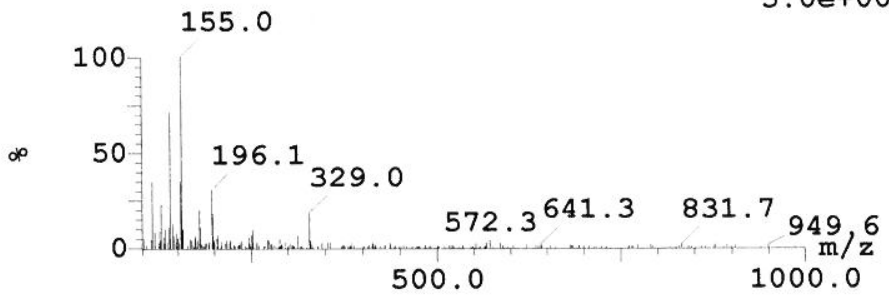

Peak ID Compound Time Mass Found 1.69

146.1

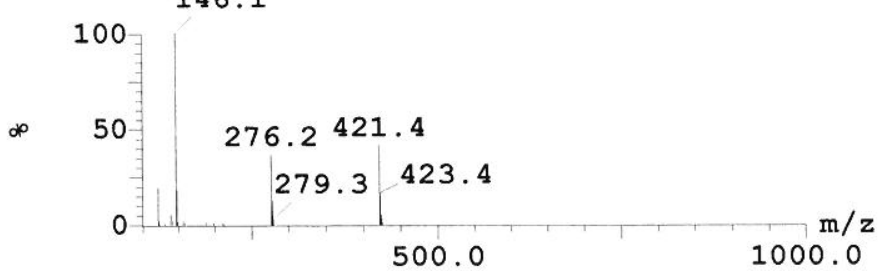

$1:$ MS ES+
$5.0 e+005$

:MS ES+
$3.912 \mathrm{e}+1$

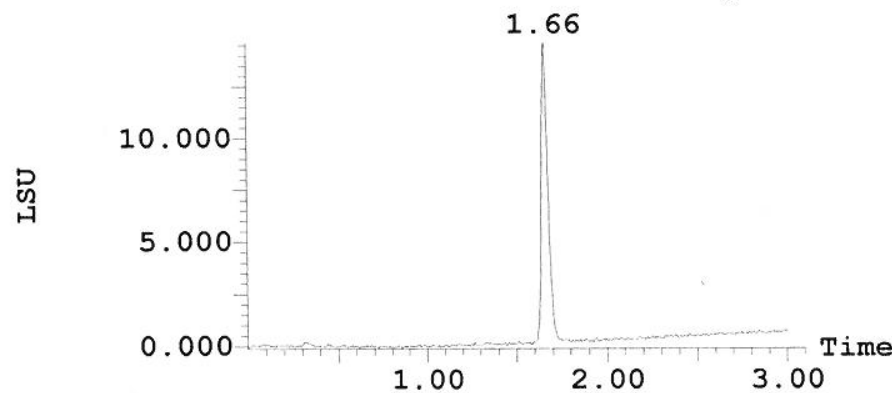

\section{(2) ELSD Signal}

3.00
1.98

2.33

0.90

4.633

Range: 14.723

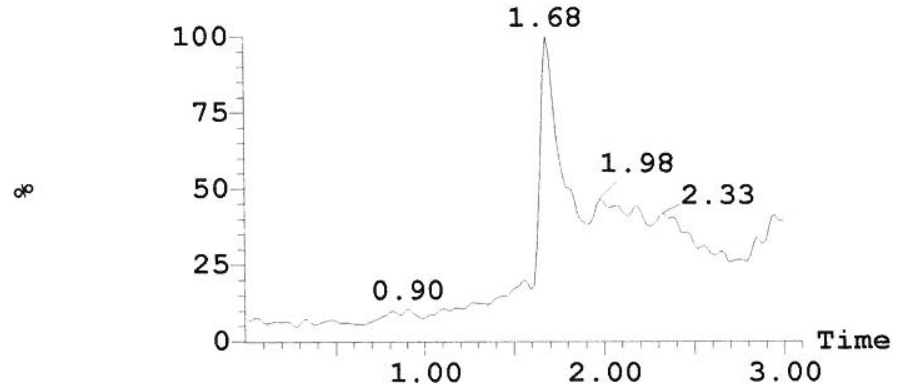

$6.8 e+006$

Peak ID Compound Time Mass Found

$1:$ MS ES+

1. $4 \mathrm{e}+006$

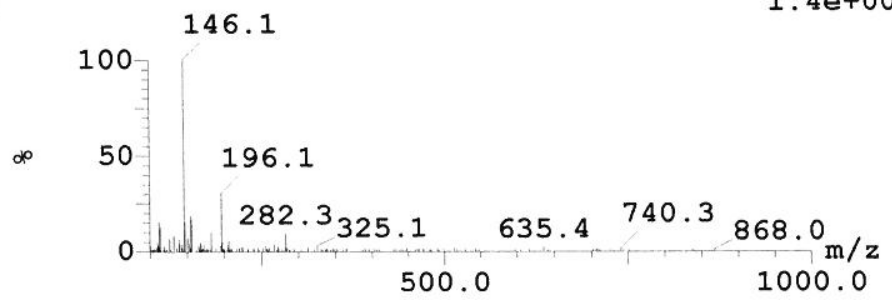

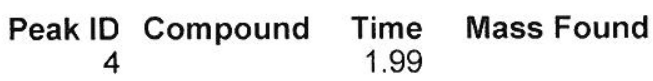

1 :MS ES+

2. $0 e+006$

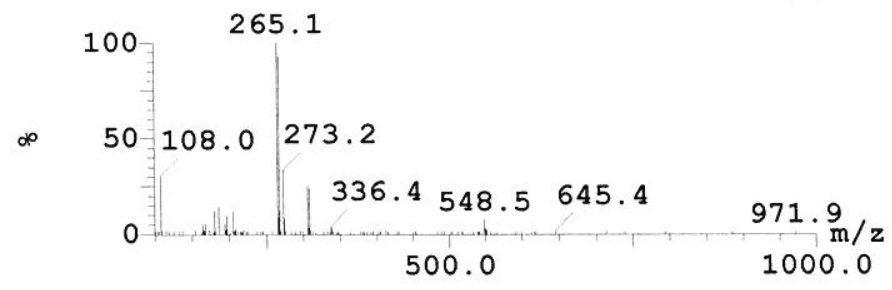

S64 
Openlynx Report - MMH

Sample: 1

File:mmh-0225-36B-1

Description:
Method:C:MassLynxiStandard Run.olp

Date:13-Nov-2016
Vial: $1: 21$

Time:22:05:10
Page 1
1: MS ES+ :TIC Smooth (SG, 2x2)

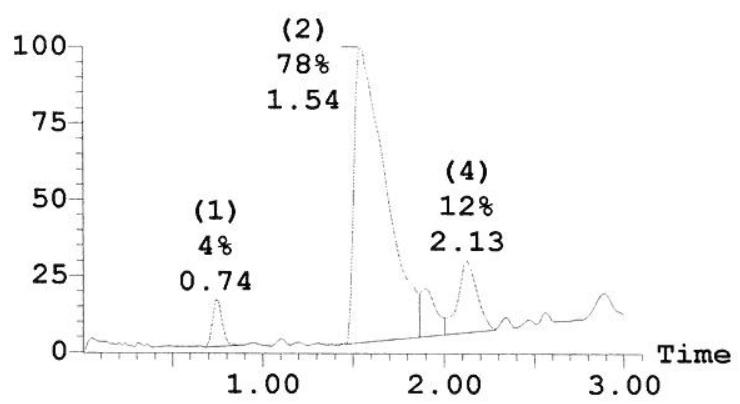

3: UV Detector: TIC

3. $325 e+2$ Range: $3.454 \mathrm{e}+2$

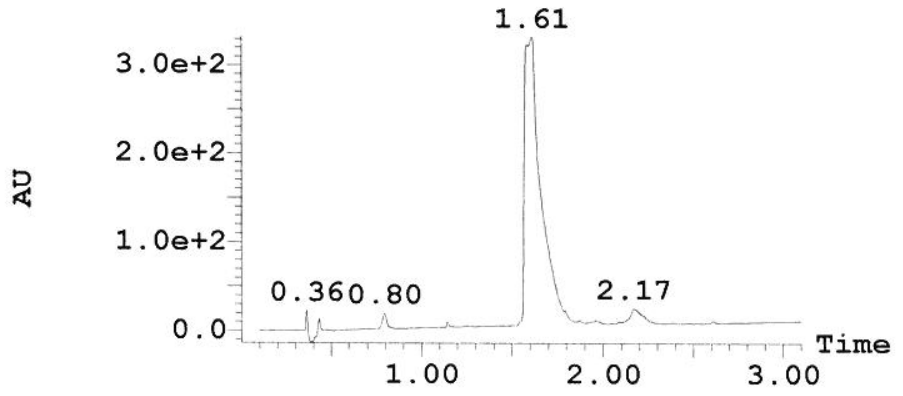

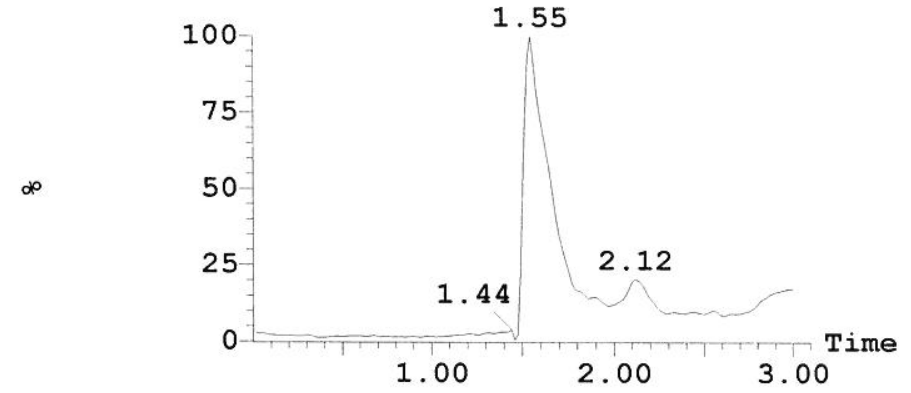

(1) ELSD Signal

1992.885 Range: 1992.985

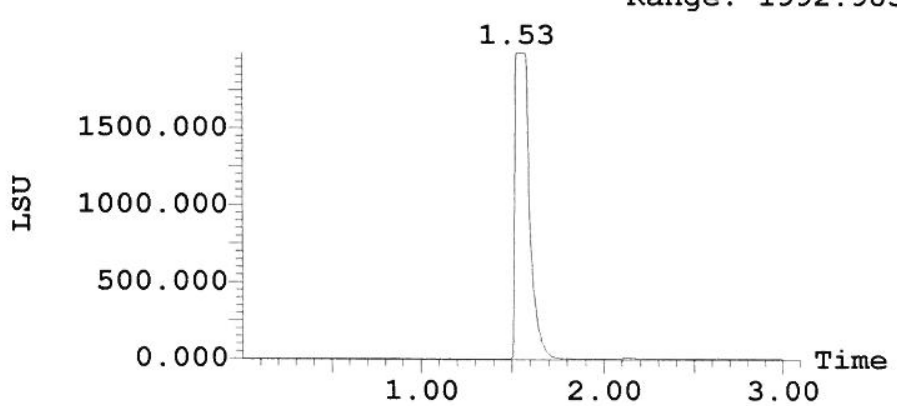

$\begin{array}{rrr}\text { Peak ID Compound } & \text { Time } \\ 1 & 0.74 & \text { Mass Found }\end{array}$

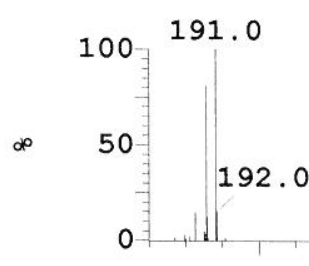

Peak ID Compound Time Mass Found $\begin{array}{rr}\text { Peak ID Compound Time } & \text { Mass Found } \\ 2 & 1.54\end{array}$

1 :MS ES+

3. $8 e+007$

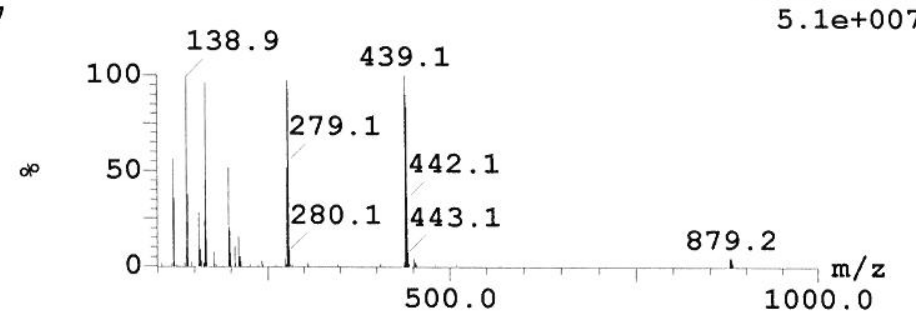

Peak ID Compound Time Mass Found
$1:$ MS ES+
$8.0 e+006$
2.13

1:MS ES+ 3. $1 e+007$

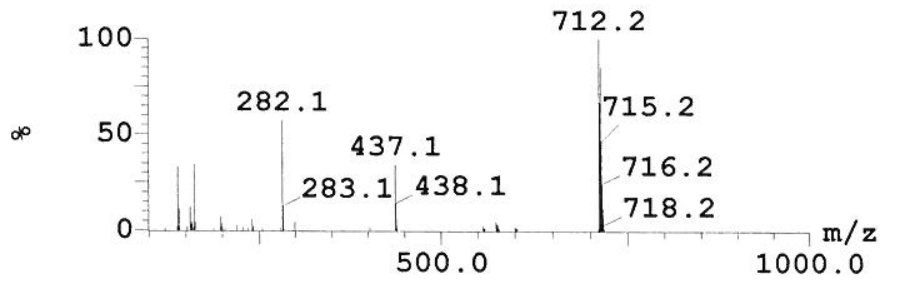


Openlynx Report -

Sample: 1

Date:03-Jan-2017

File:tjm-238-06-310
Method:C:IMassLynxIStandard_Run.olp

Time:15:11:12
Vial:1:16
Description:

Page 1
1: MS ES+ :TIC Smooth (SG, 2x2)

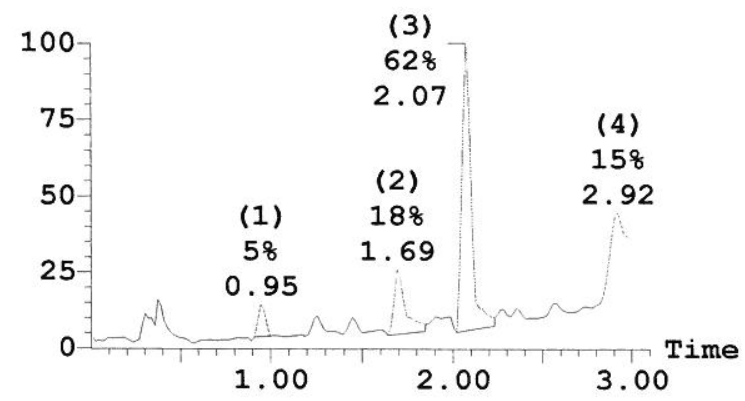

3: UV Detector: TIC

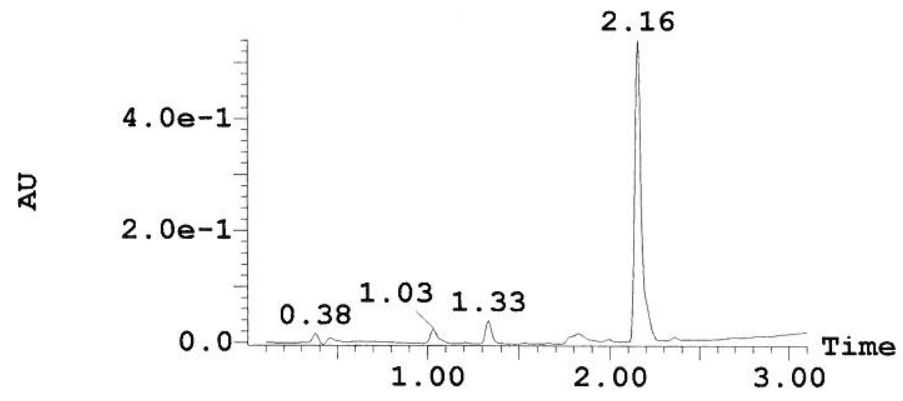

Peak ID Compound Time Mass Found

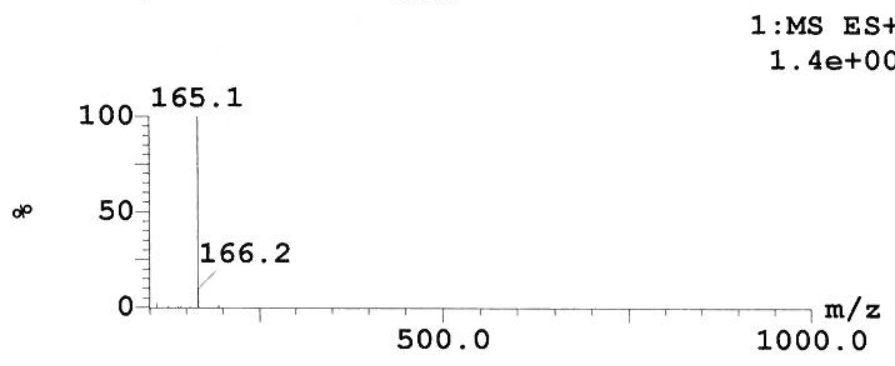

Peak ID Compound Time Mass Found

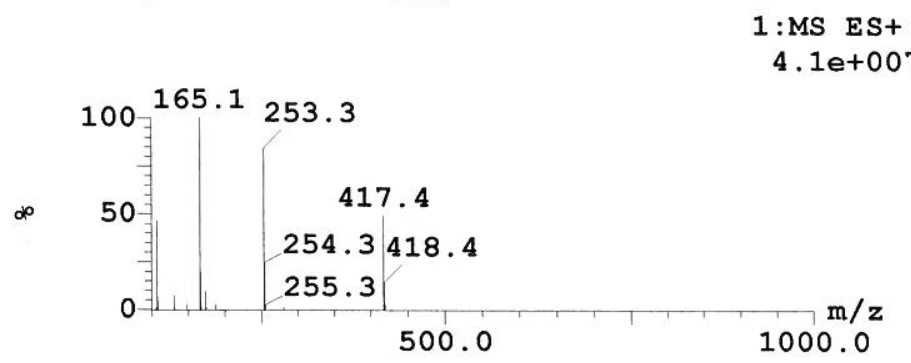

Peak ID Compound $\begin{array}{r}\text { Time } \\ 2\end{array} \quad$ Mass Found
1.69

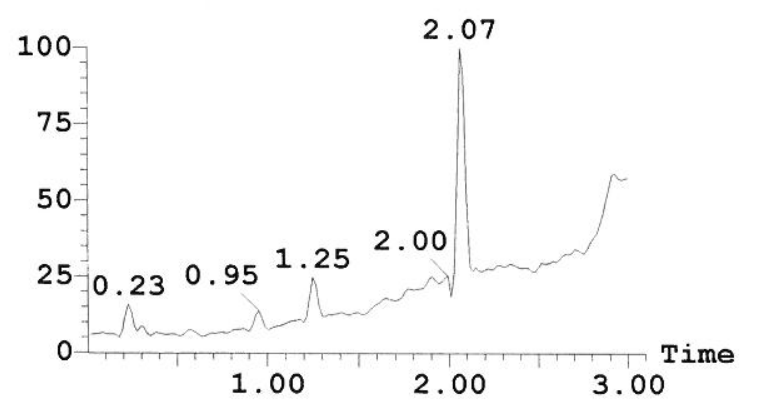

(2) ELSD Signal

4.257

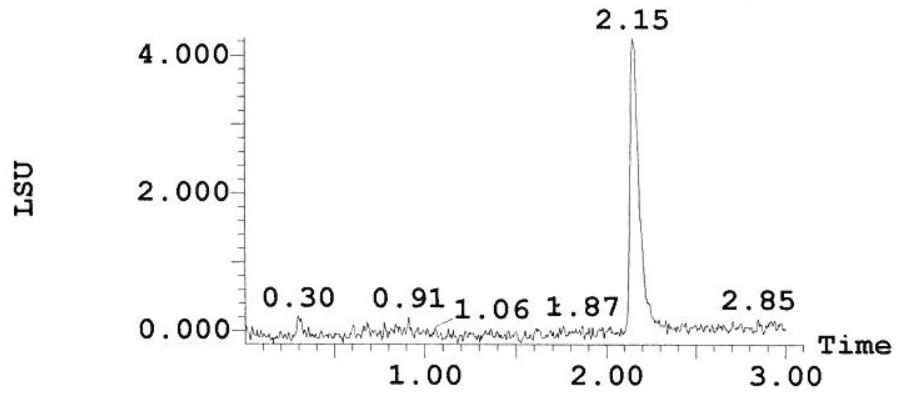


Openlynx Report - MSH

Sample: 1

File:msh-0224-57 f30

Description:
Method:C:MassLynxiStandard Run.olp Date:12-Dec-2016
Vial:2:38

Time:10:59:34
1: MS ES+ :TIC Smooth (SG, 2x2)

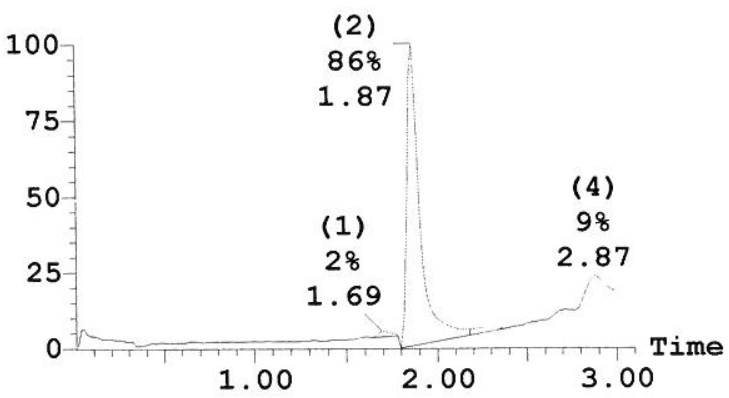

3: UV Detector: TIC

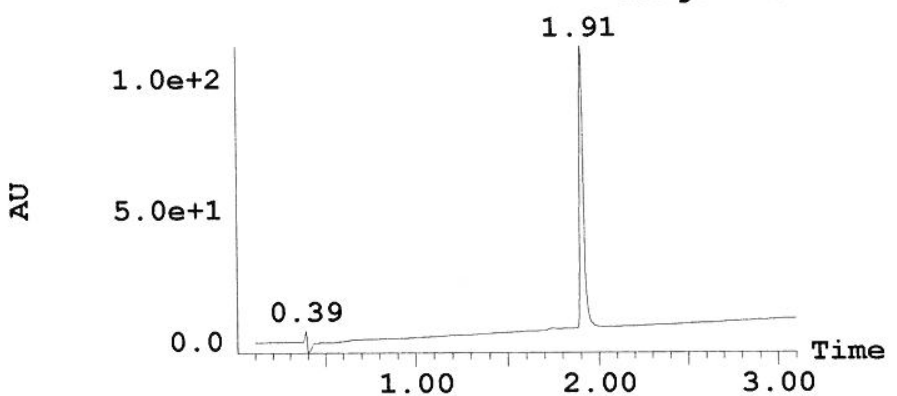

Peak ID Compound Time Mass Found

$1.127 e+2$ ange: $1.165 e+2$

ELSD Signal

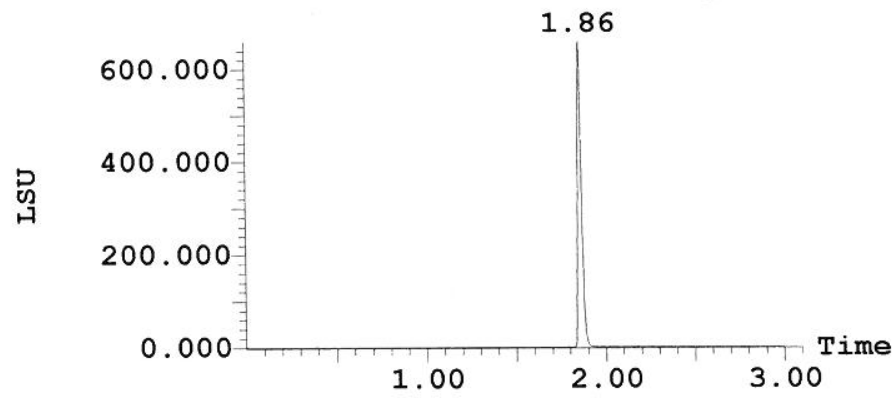
1.69

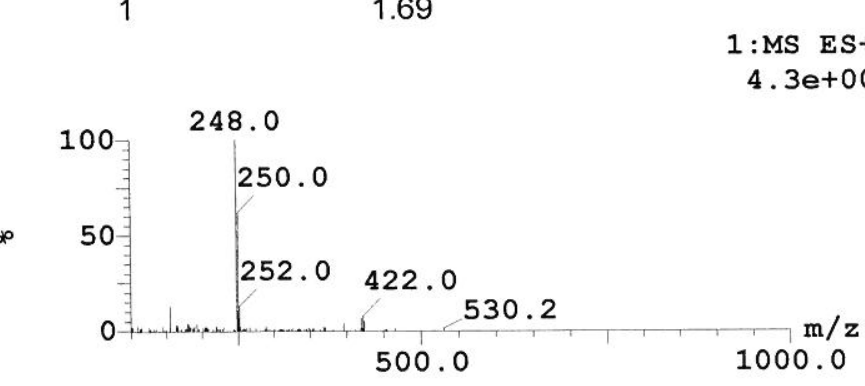

Peak ID Compound Time Mass Found 2.25 1000.0

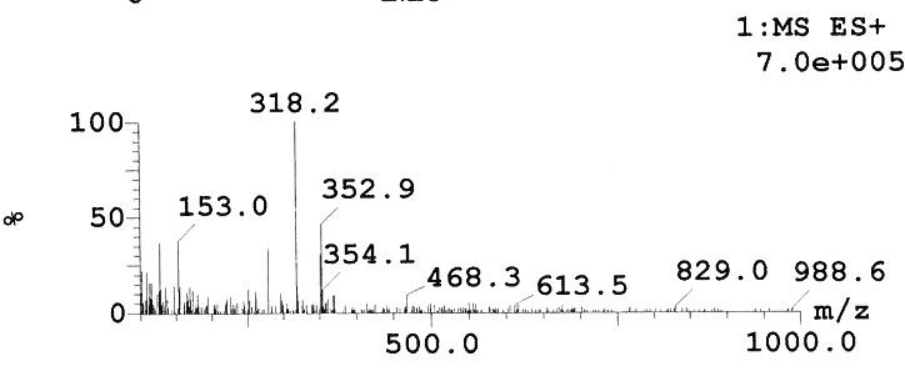

\section{Peak ID Compound Time Mass Found}

$1:$ MS ES+

$6.0 e+007$

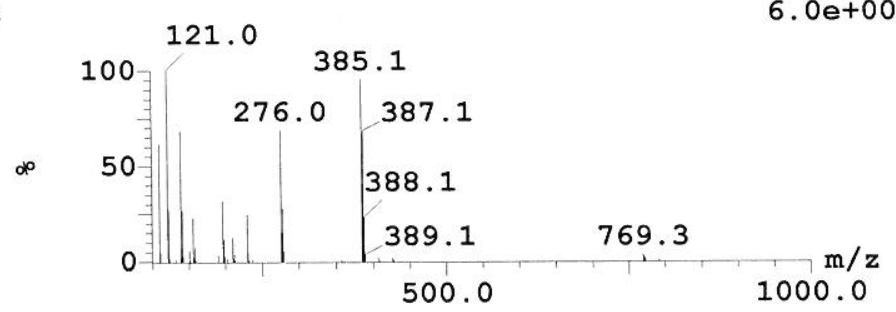

$\begin{array}{rr}\text { Peak ID Compound } & \text { Time } \\ 4 & 2.87\end{array}$ Mass Found

$1:$ MS ES+ $1.2 e+007$

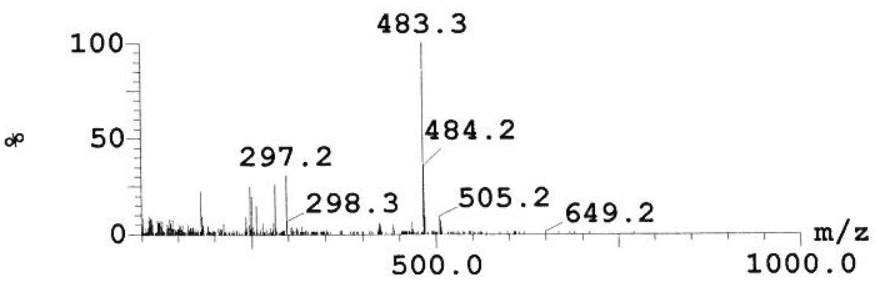


Vial:1:25

Time:14:59:28

Description:

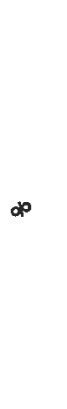

$5.5 e+007$

1: MS ESt :TIC Smooth (SG, 2×2)

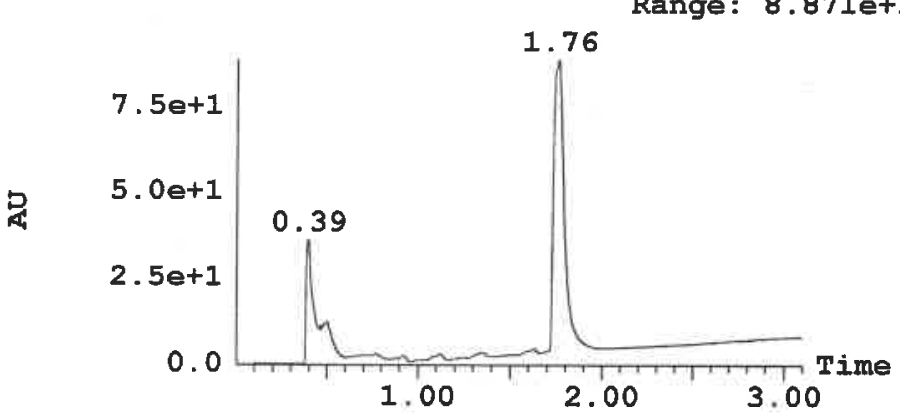

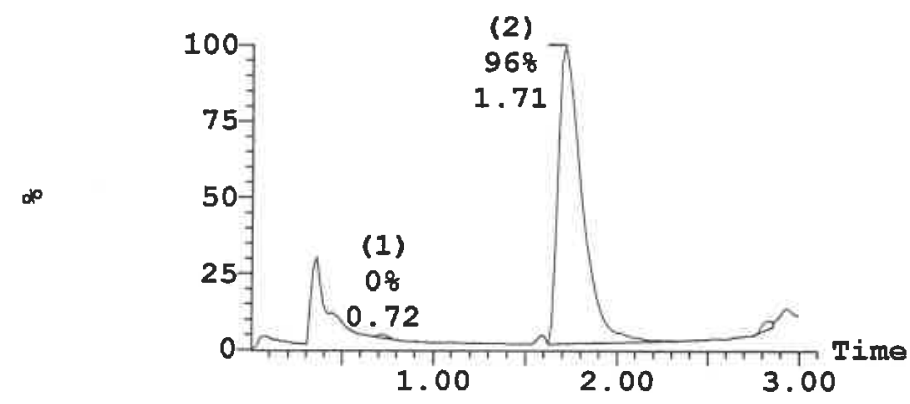

3: UV Detector: TIC

Peak ID Compound $\begin{array}{r}\text { Time } \\ 1\end{array} \quad$ Mass Found
0.72

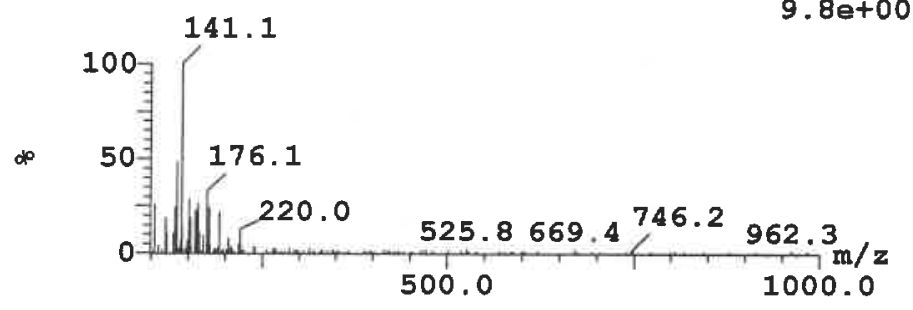

Peak ID Compound Time Mass Found 2.82

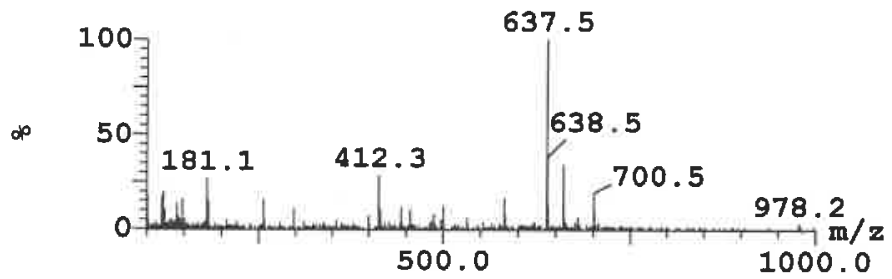

(1) ELSD Signal

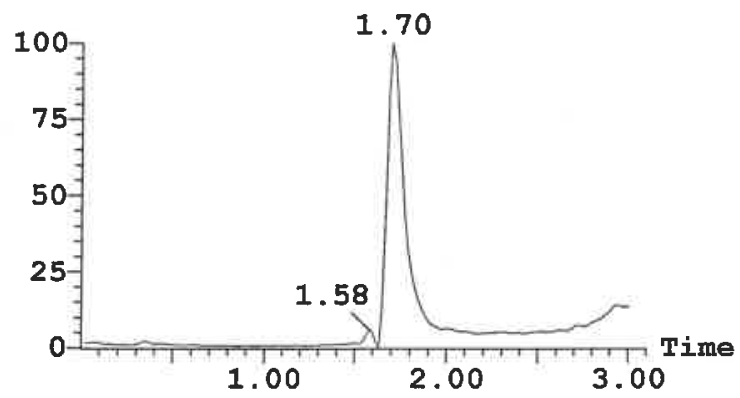

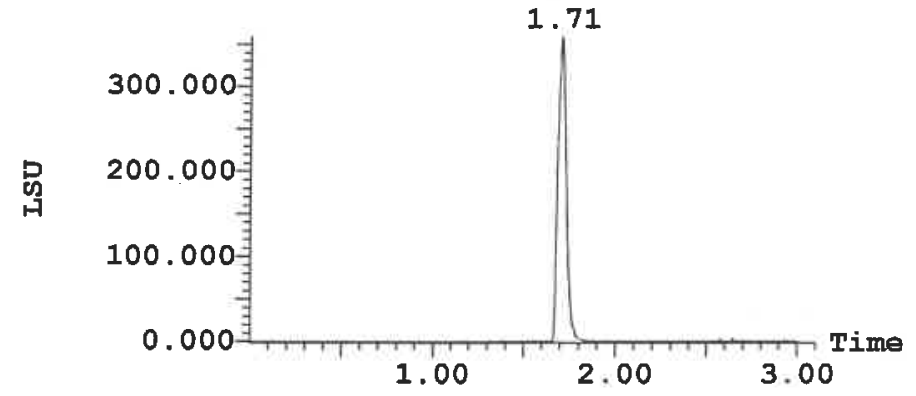

\section{$\underset{2}{\operatorname{Peak} \text { ID Compound }} \begin{array}{r}\text { Time } \\ 1.71\end{array}$ Mass Found}

1:MS ES+ $9.8 e+005$

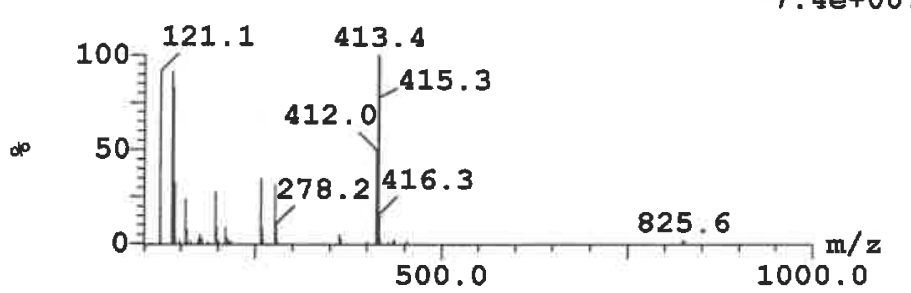

$\begin{array}{rr}\text { Peak ID Compound } & \text { Time } \\ 4 & 2.93\end{array}$ Mass Found

$1:$ MS ES+
$3.3 e+006$

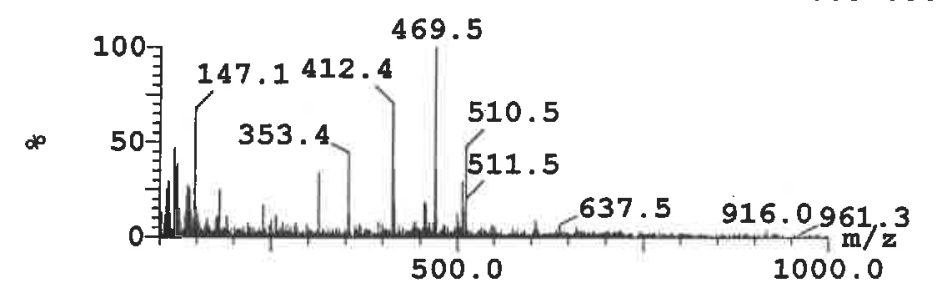


Method:C:MassLynx\Standard_Run.olp Time:09:58:24
Page 1

Vial:2:13

Description:
1: MS ES+ :TIC Smooth (SG, 2×2)

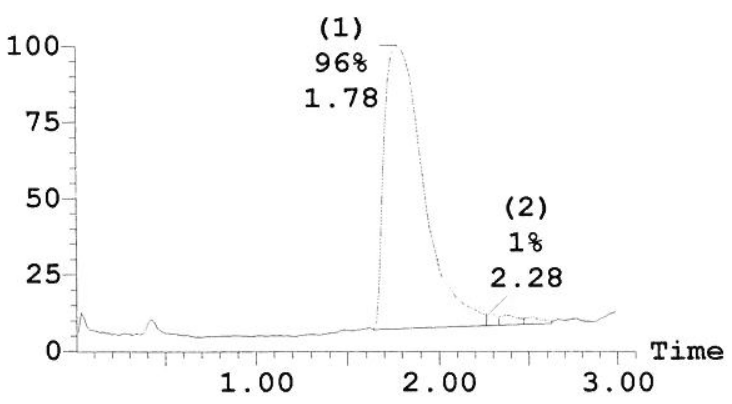

3: UV Detector: TIC

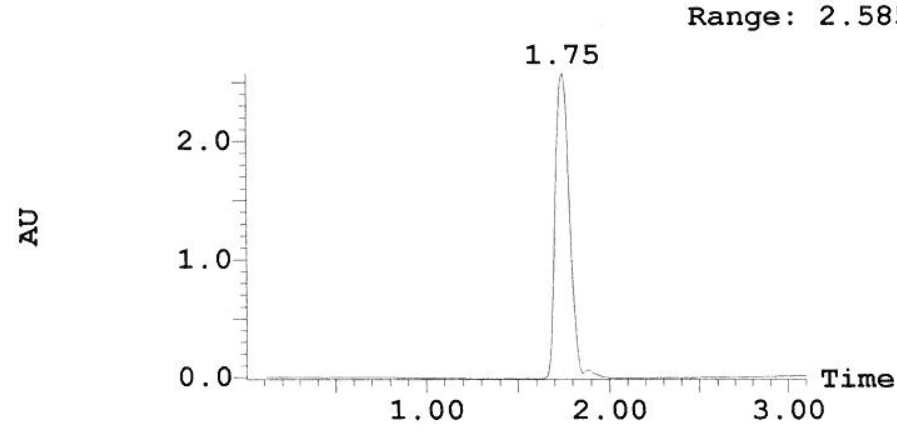

3.8e+008 2: MS ES- :TIC Smooth (SG, 2x2)

3. $9 e+007$

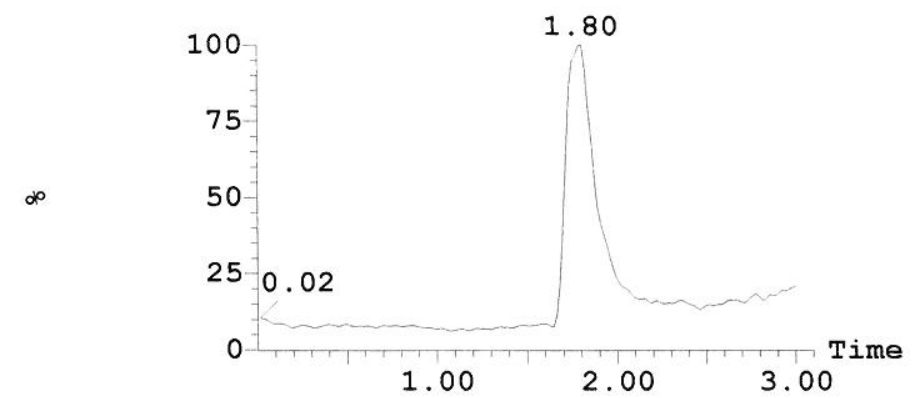

(2) ELSD Signal

1986.565

Range: 2013.304

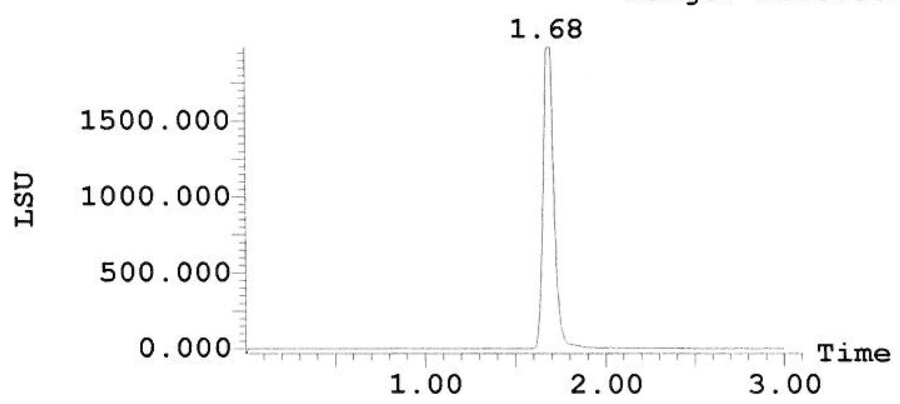

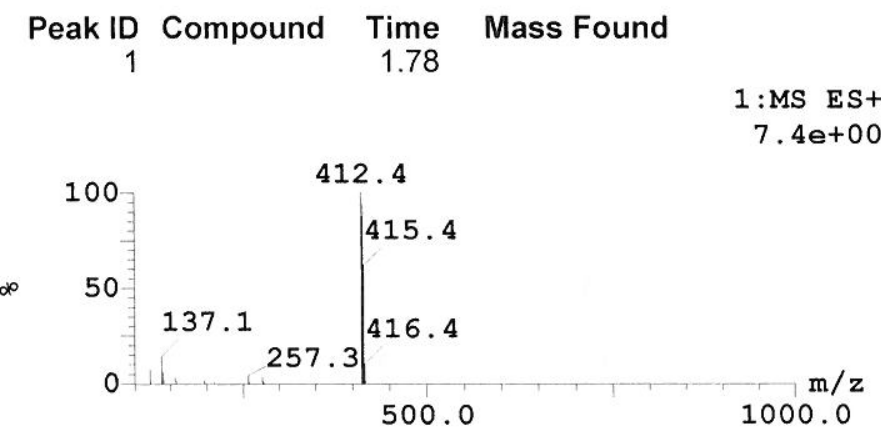

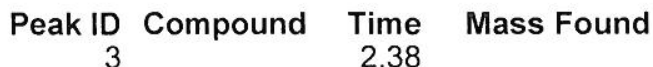

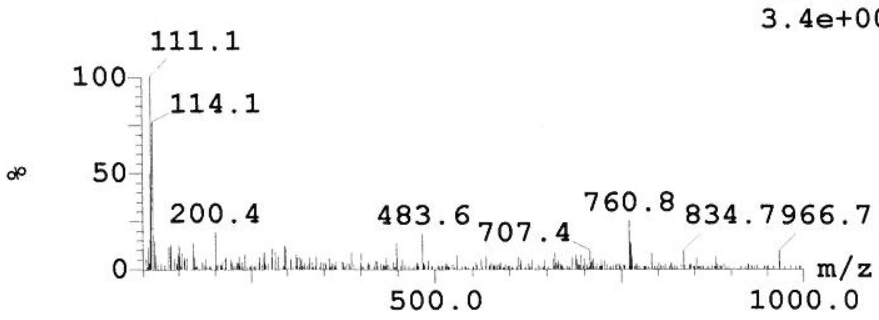

Peak ID Compound Time Mass Found

2.28

$1:$ MS ES+

2. $4 e+005$

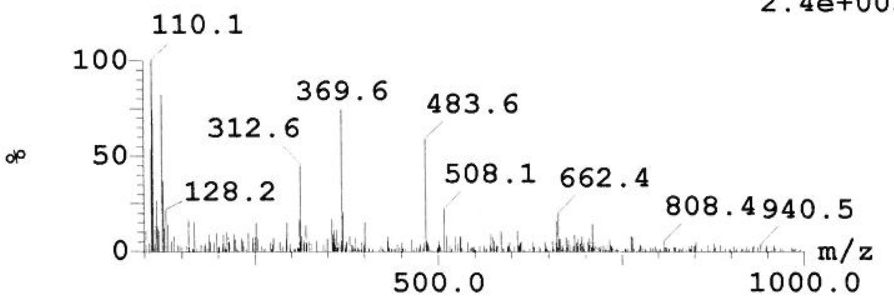

\section{Peak ID Compound Time
4}

$1:$ MS ES +

2. $2 e+005$

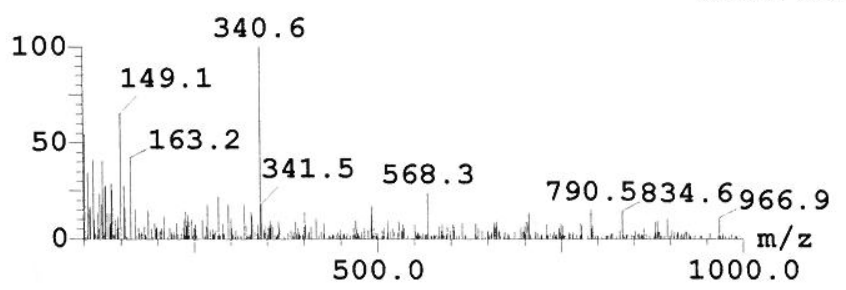


Vial:1:8

Time:10:37:04

\section{Description:}

1: MS ES+ :TIC Smooth (SG, 2×2)

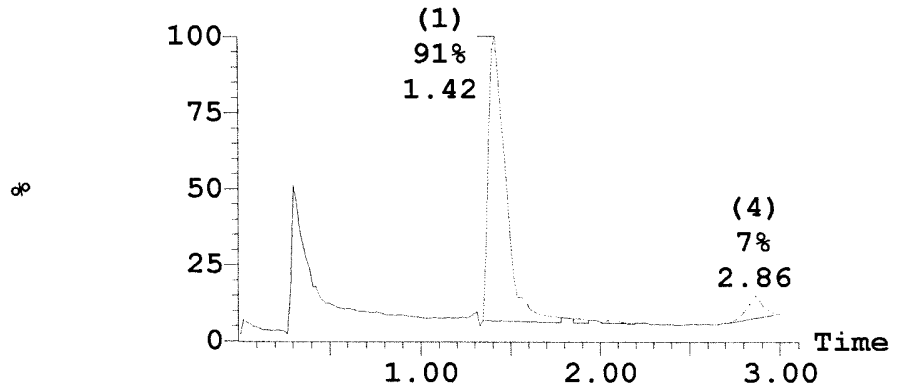

3: UV Detector: TIC

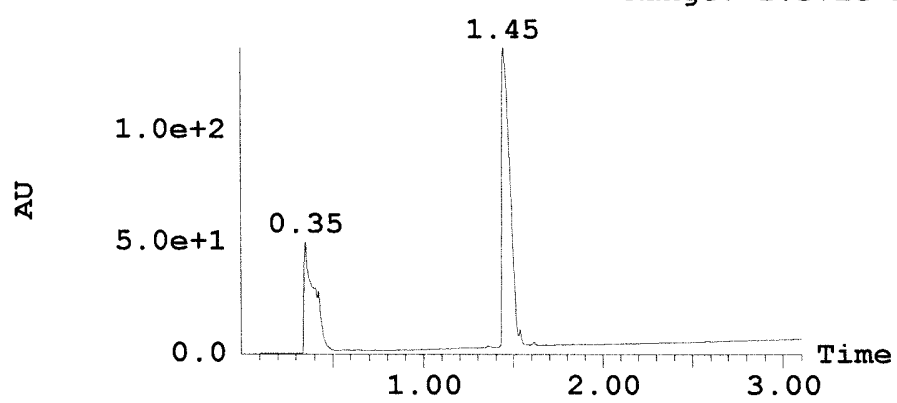

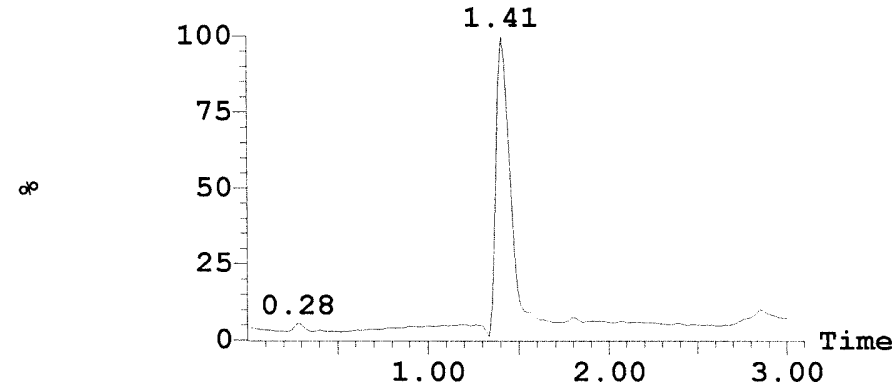

(1) ELSD Signal

645.706

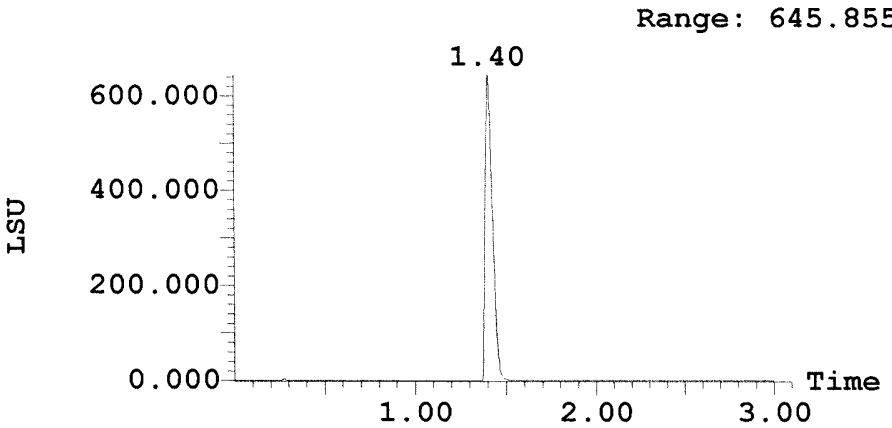

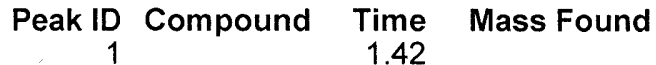

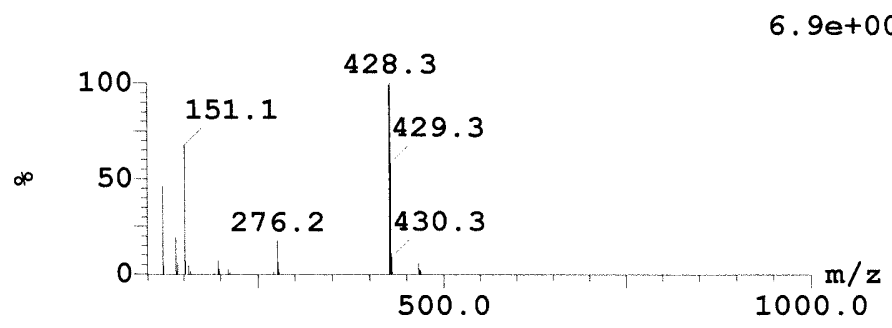

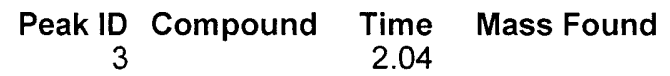

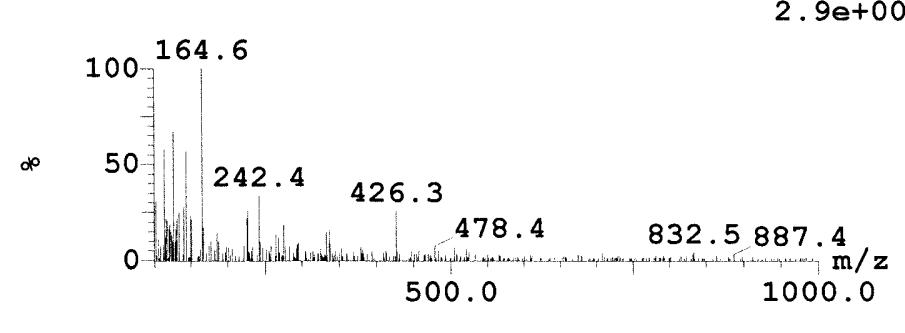

\section{Peak ID Compound Time Mass Found}

1:MS ES+

$2.7 e+005$

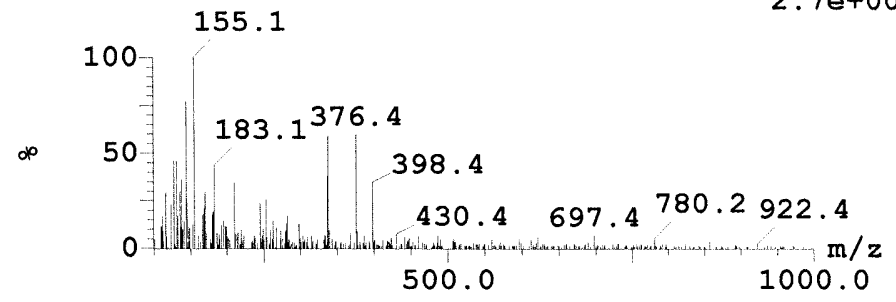

Peak ID Compound Time Mass Found

$1:$ MS ES+

2. $1 e+006$

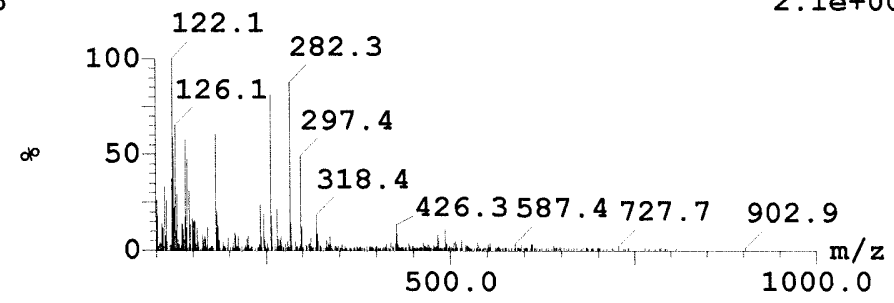


Vial: $1: 4$

Description:
1: MS ES+ :TIC Smooth (SG, 2x2)

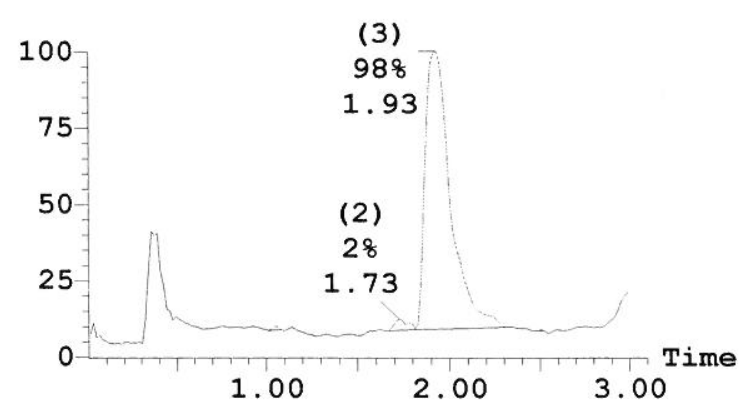

3: UV Detector: TIC

2.749

Range: 2.778

1.97

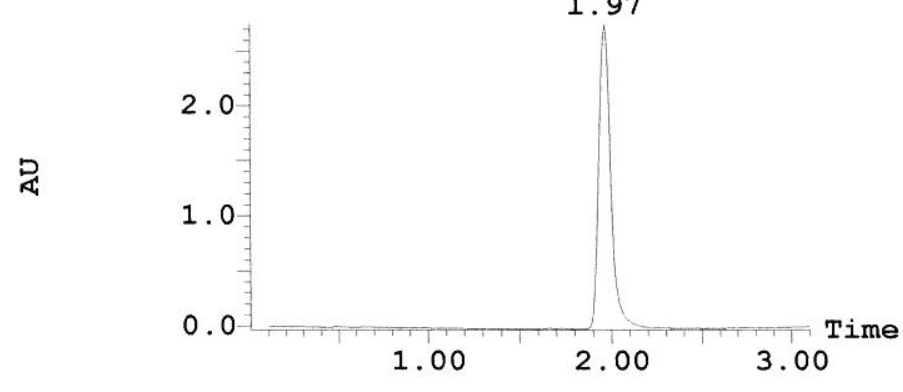

Peak ID Compound Time
1 $\quad$ Mass Found

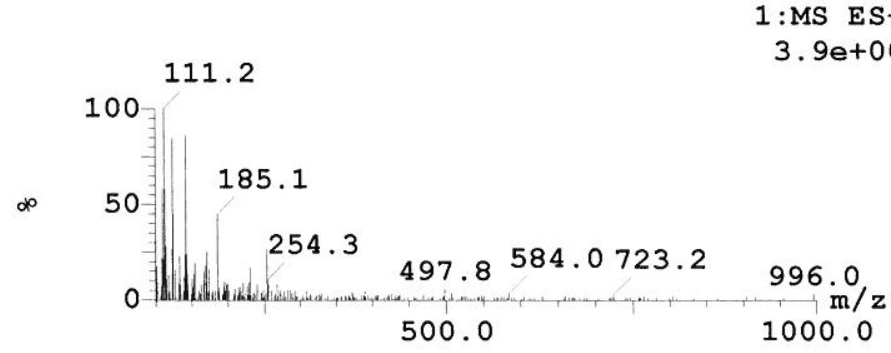

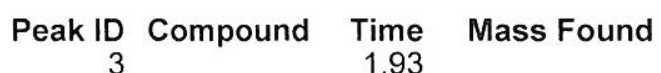

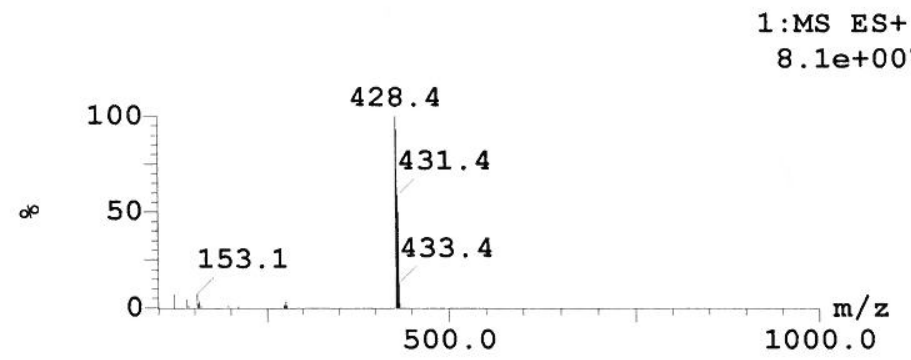

2: MS ES- :TIC Smooth (SG, 2×2)

$2.7 e+007$

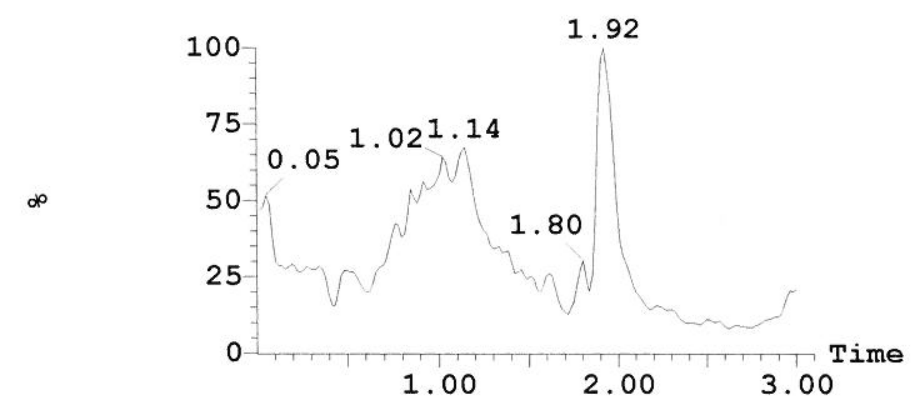

(2) ELSD Signal

1816.114

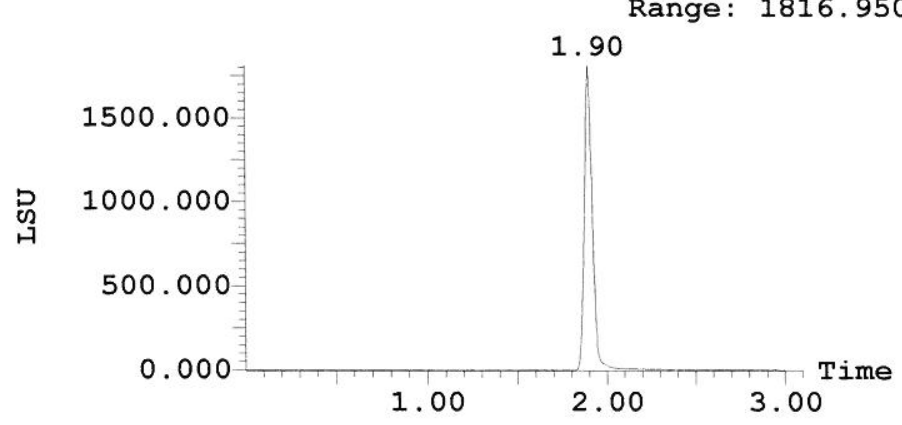


Openlynx Report -

Sample: 1

Date:01-Aug-2017

File:jpb264-19 on
Method:C:MassLynxiStandard_Run.olp Time:08:50:03
Page 1

Vial:2:31

Description:
1: MS ESt :TIC Smooth (SG, $2 \times 2$ )

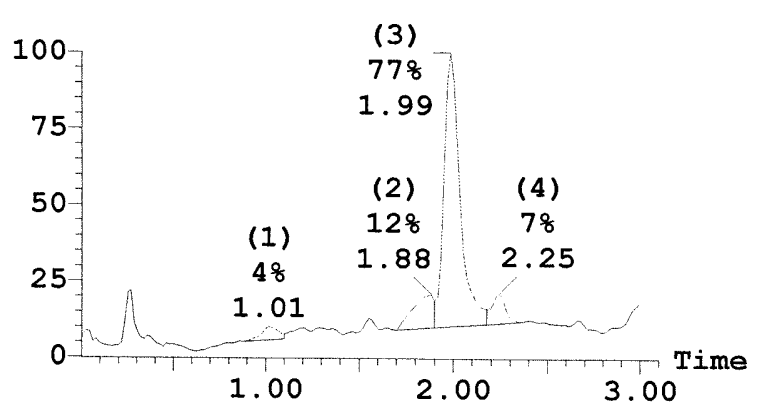

3: UV Detector: TIC

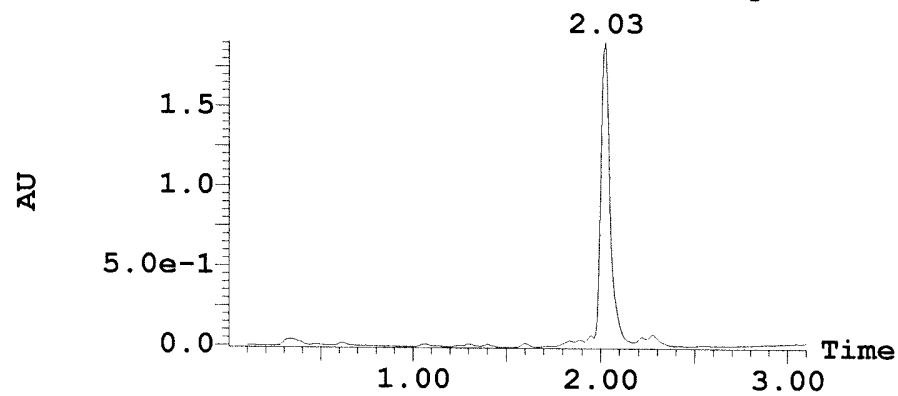

Peak ID Compound Time Mass Found

131.2

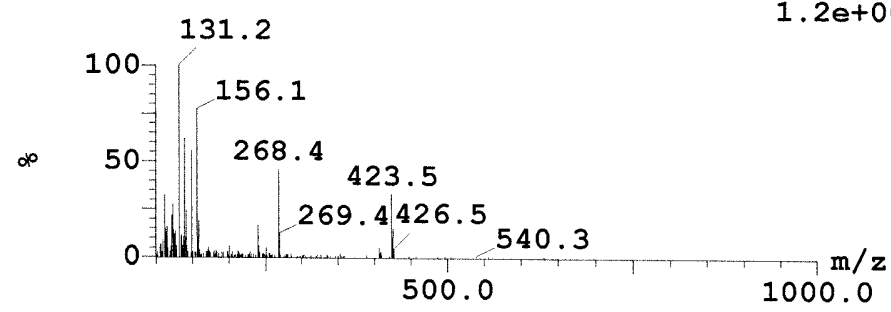

Peak ID Compound Time Mass Found

$3 \quad 1.99$

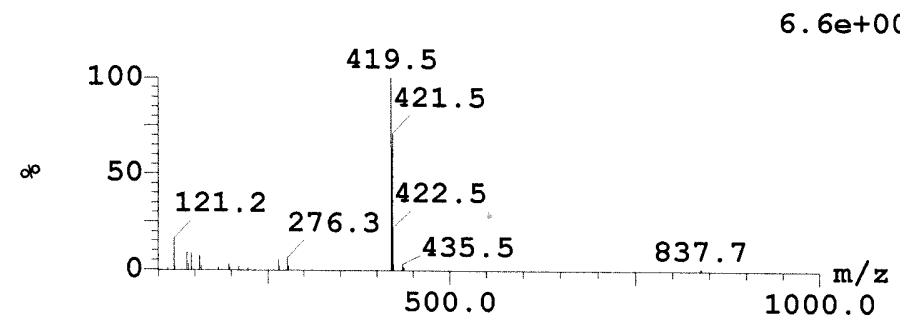

1.912

1.918

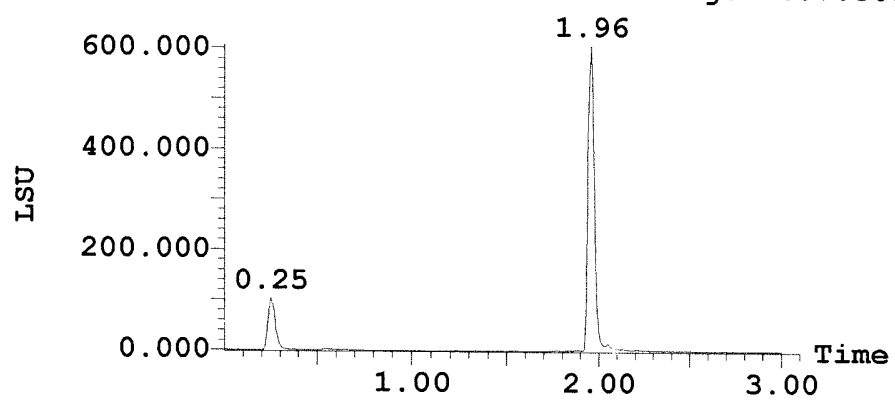

(2) ELSD Signal

\section{Peak ID Compound
2 $\begin{array}{r}1.88 \\ \text { Time }\end{array}$}

$1:$ MS ES+

: MS ES- :TIC Smooth (SG, $2 \times 2$ )

$2.4 e+007$

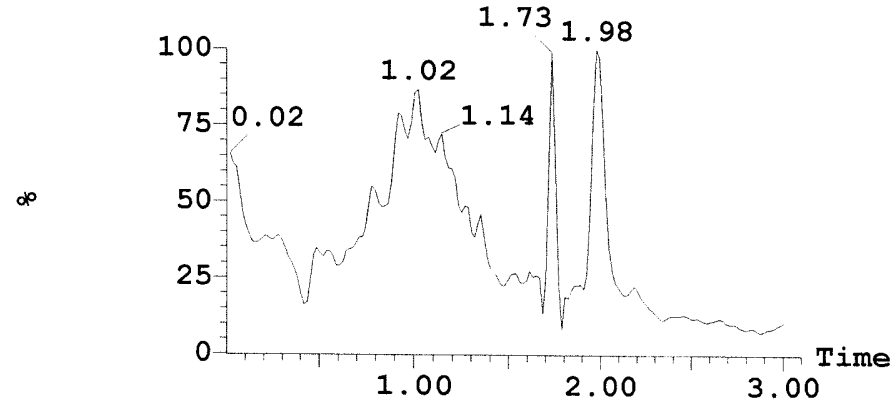

1.00

606.944

07.509

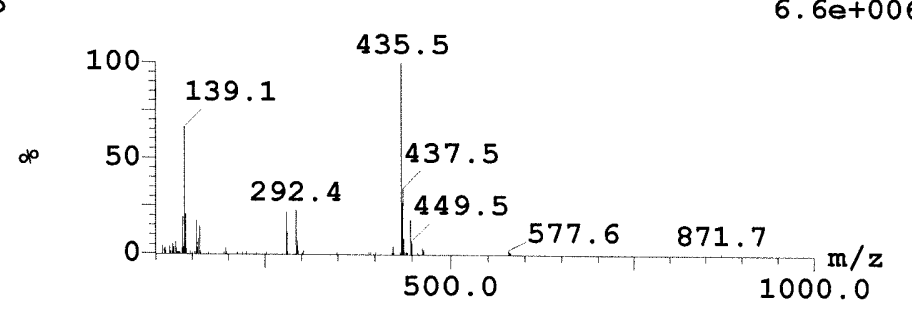

Peak ID Compound Time
4

$1:$ MS ES+

$6.6 e+007$

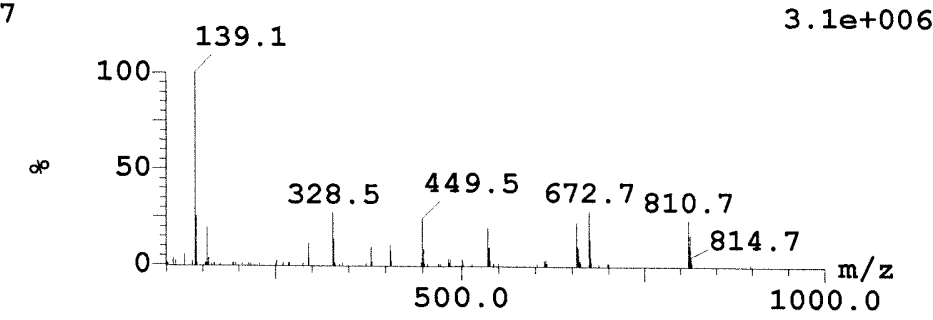


Vial:1:30

Time:10:57:47

\section{Description:}

1: MS ES+ : TIC Smooth (SG, $2 \times 2$ )

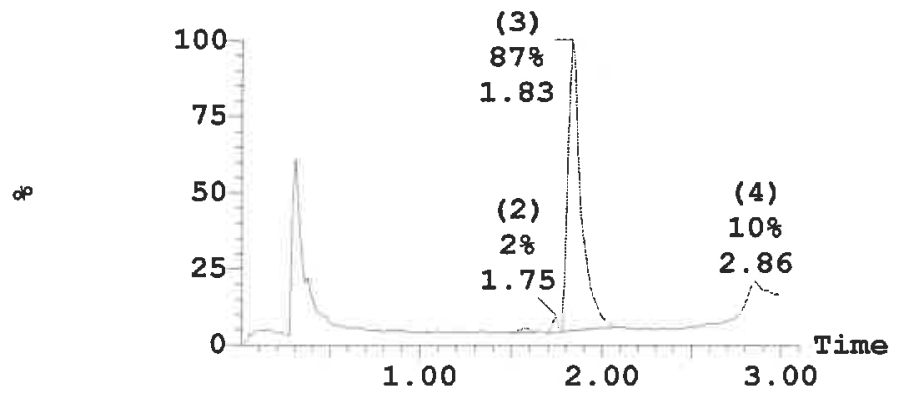

3: UV Detector: TIC

$1.621 \mathrm{e}+2$
$7.2 \mathrm{e}+008$ 2: MS ES- :TIC Smooth (SG, $2 \times 2$ )

$4.5 e+007$

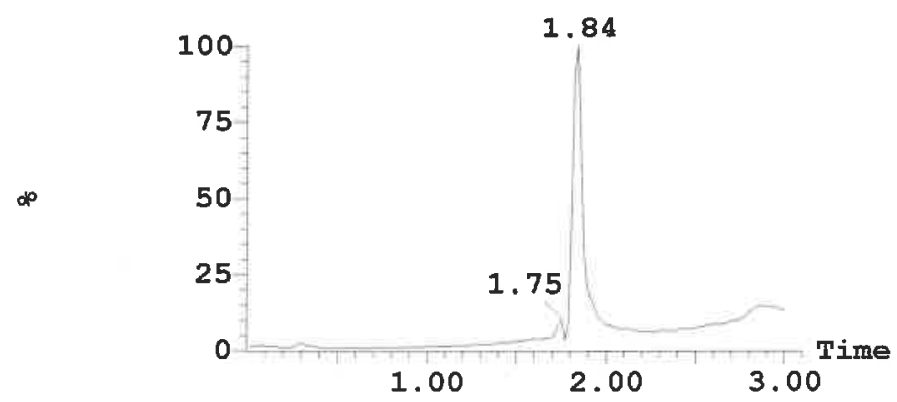

(1) ELSD Signal

539.898 Range: $1.625 \mathrm{e}+2$ 1.89
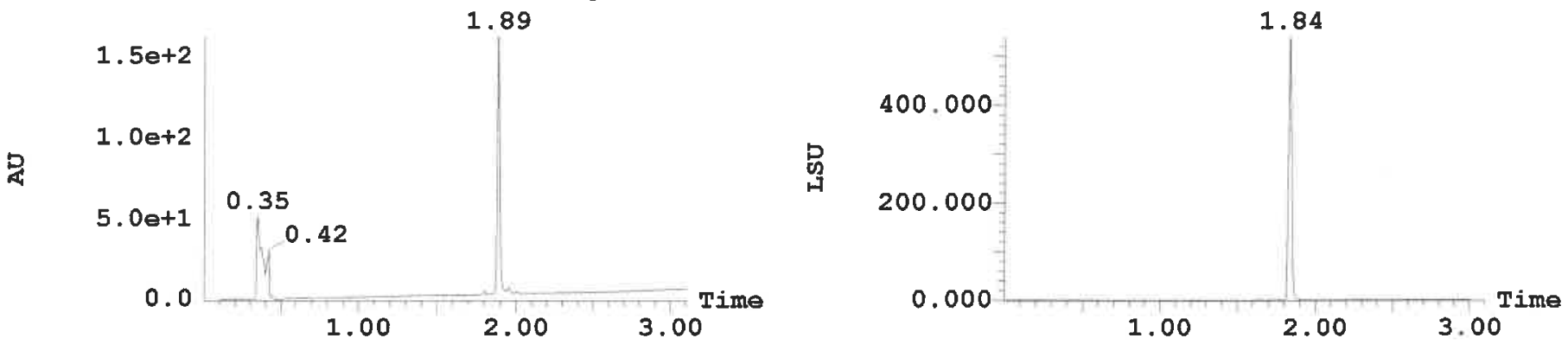

Peak ID Compound Time Mass Found

Peak ID Compound Time Mass Found

:MS ES+

$1.1 e+006$
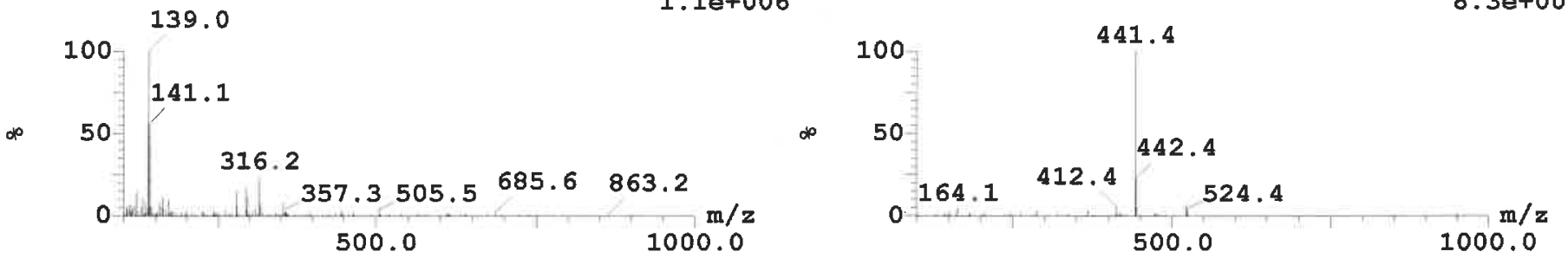

Peak ID Compound Time
3 $\quad$ Mass Found

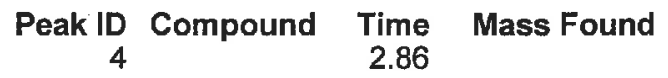

$1:$ MS ES+

5. $7 e+007$

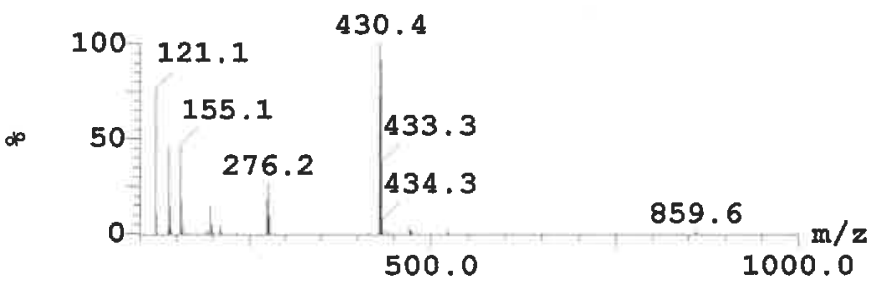

1 :MS ES+

8. $3 e+006$

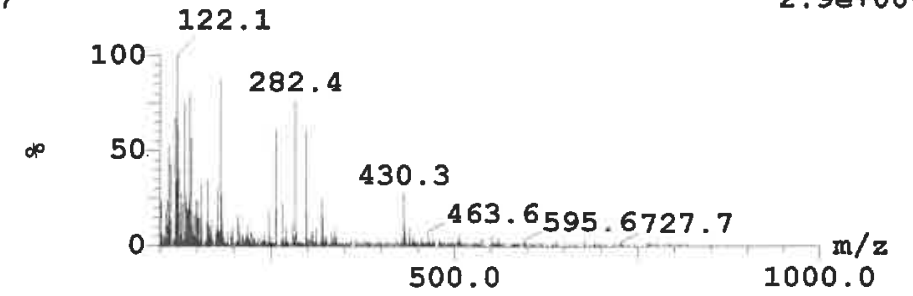


Openlynx Report - MMH

Sample: 1

File:mmh-70141-0-2

Description:
Method:C:MassLynxiStandard Run.olp

Date:17-Jun-2021
Vial: $1: 29$

Time:15:17:08
Page 1
1: MS ESt :TIC Smooth (SG, 2x2)

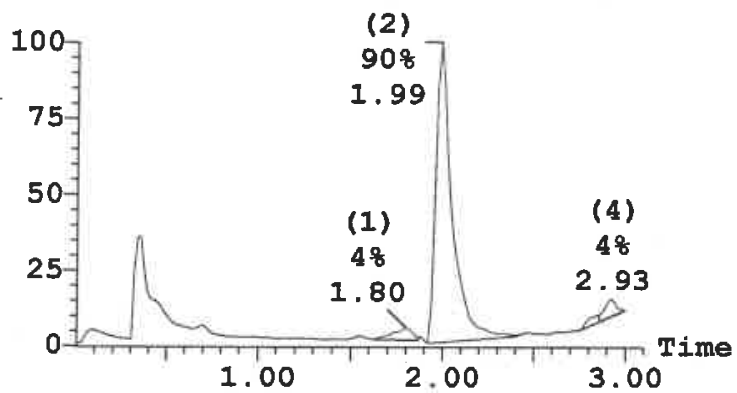

3: UV Detector: TIC

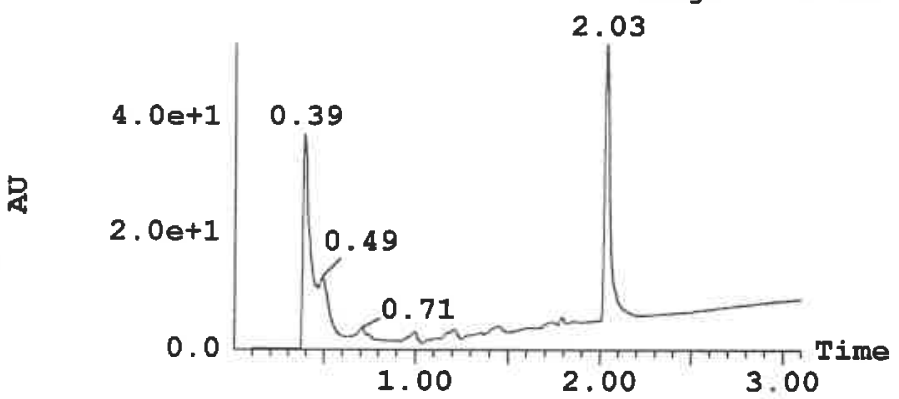

8.2e+008 2: MS ES- :TIC Smooth (SG, 2×2)

$5.8 e+007$

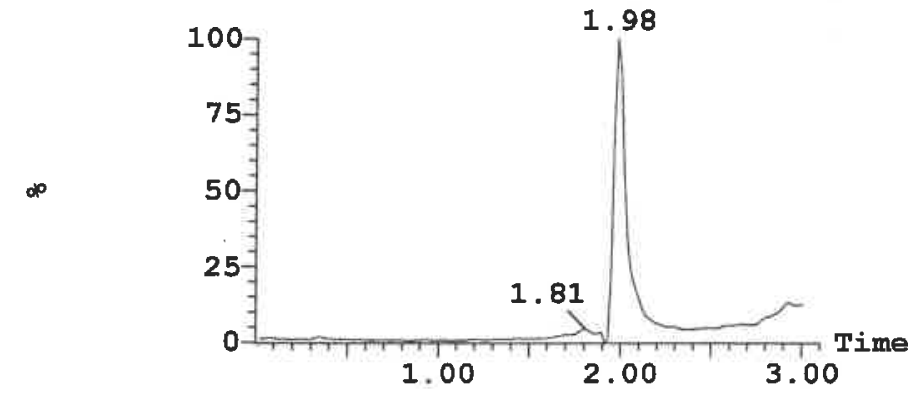

(1) ELSD Signal

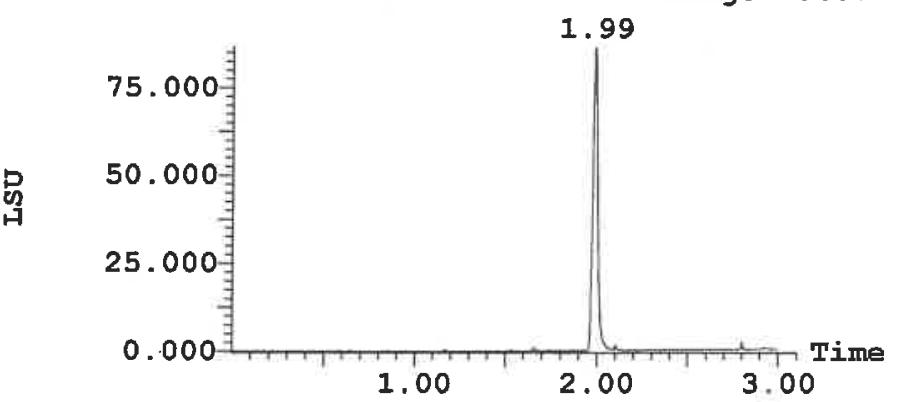

Peak ID Compound Time Mass Found

1 1.80

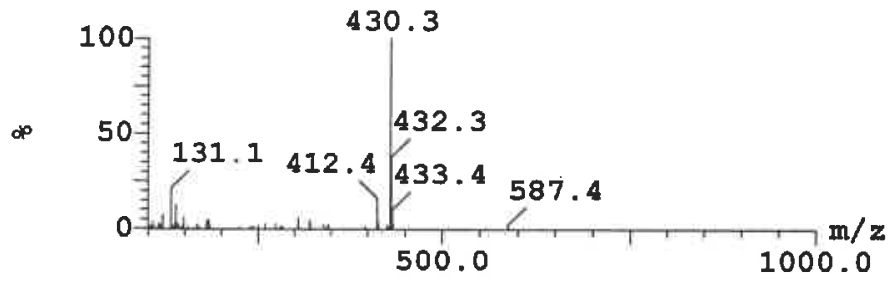

Peak ID Compound Time Mass Found 3 2.86

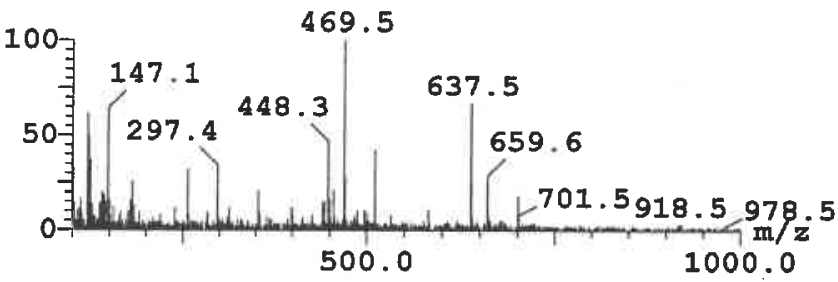

\section{Peak ID Compound
2}

1 :MS ES+ $7.0 e+007$

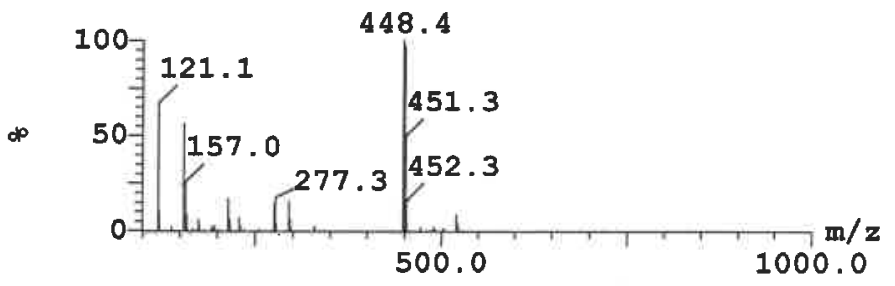

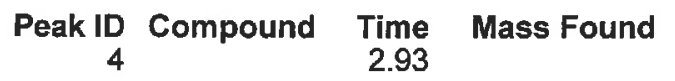

1:MS ES+ $5.4 e+006$

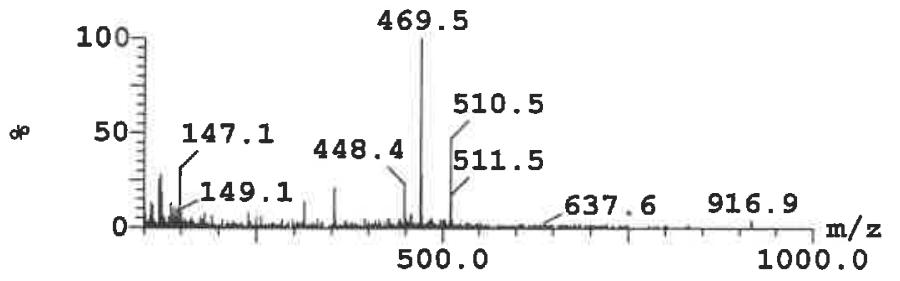


1: MS ES+ :TIC Smooth (SG, 2×2)

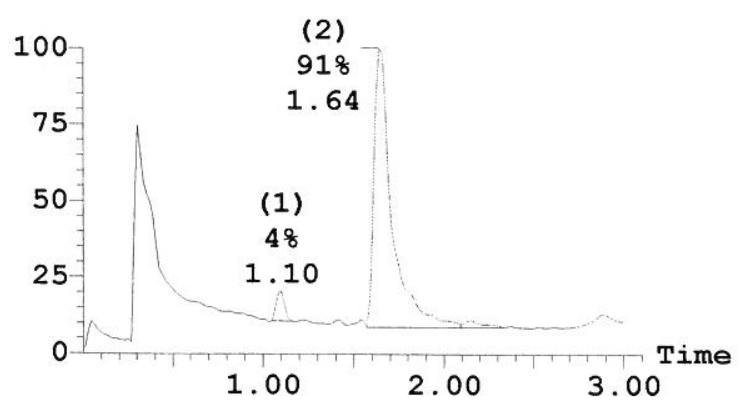

3: UV Detector: TIC

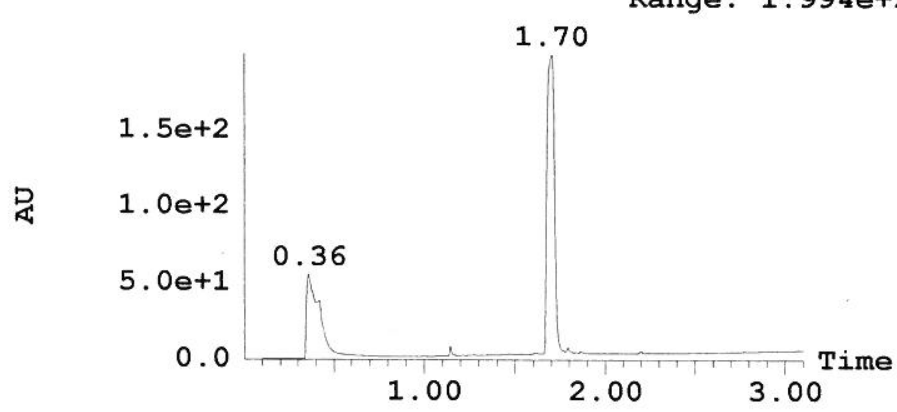

$1.994 \mathrm{e}+2$ 6.3e+008 2: MS ES- :TIC Smooth (SG, 2x2)

$9.8 e+007$

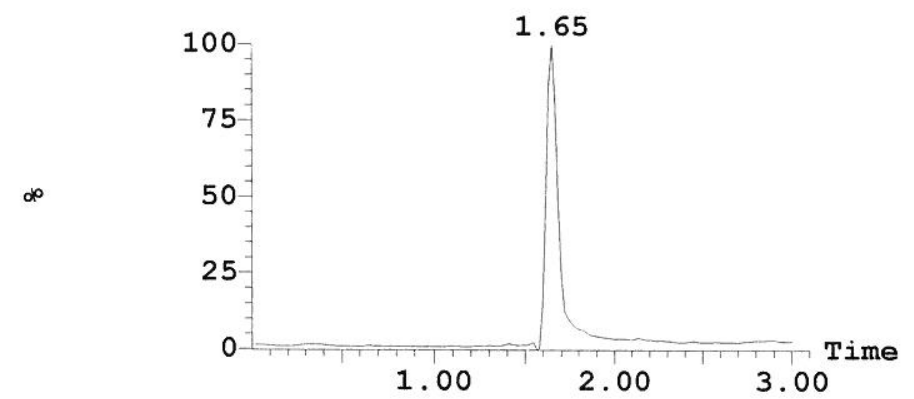

(1) ELSD Signal

1992.706

Range: 1992.905

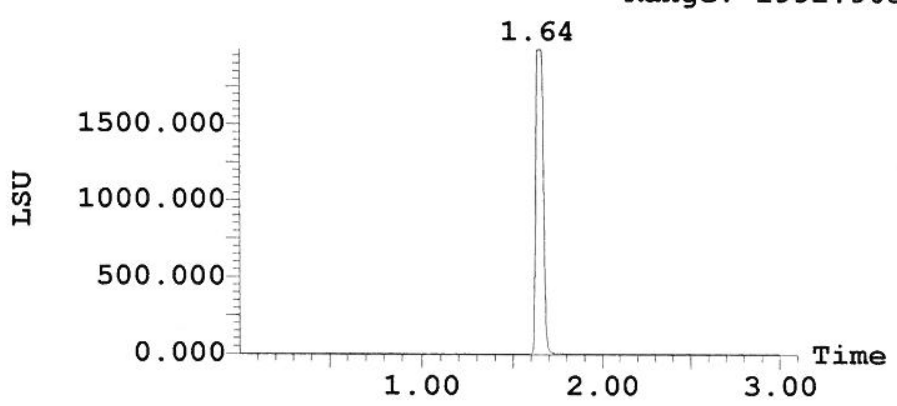

Peak ID
1 Compound $\begin{array}{r}\text { Time } \\ 1.10\end{array}$ Mass Found

$1:$ MS ES+

$1.5 e+007$

\section{Peak ID Compound Time
1.64 Mass Found}

$1:$ MS ES+

6. $9 e+007$

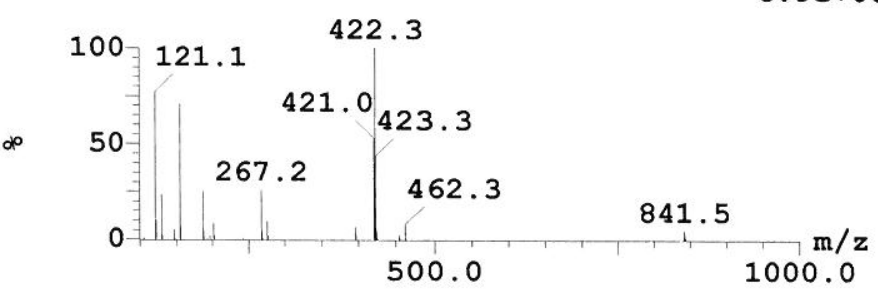

$\begin{array}{rr}\text { Peak ID Compound } & \text { Time } \\ 4 & 2.89\end{array}$

1:MS ES+

9. $6 e+005$

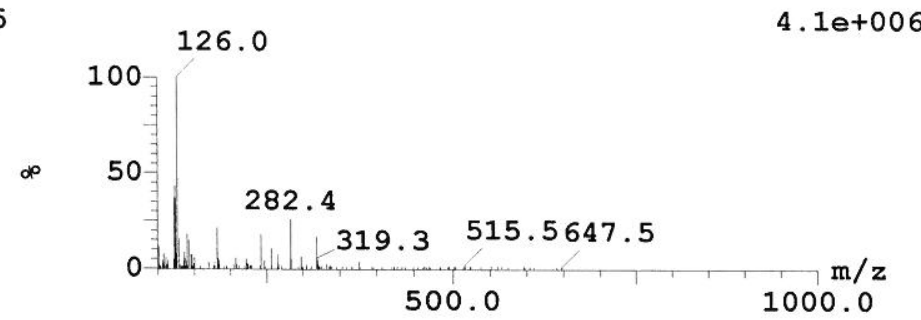


Openlynx Report -

Sample: 1

Date:02-Aug-2017

File:mmh-0266-09-X1
Method:C:MassLynxIStandard_Run.olp

Time:17:14:36
Page 1

Vial: $1: 2$

Description:
1: MS ES+ :TIC Smooth (SG, 2x2)

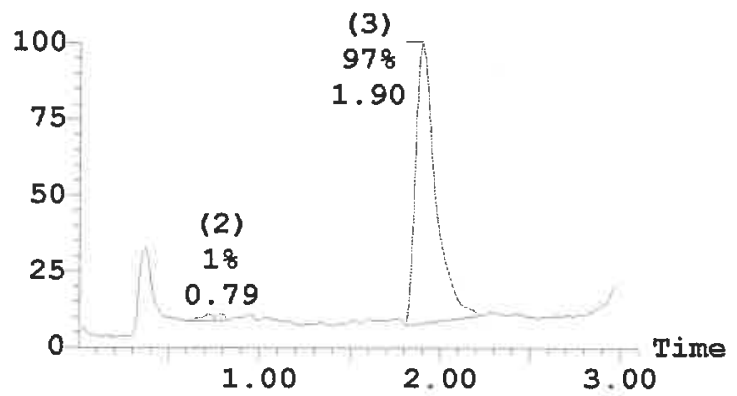

4.1e+008 2: MS ES- :TIC Smooth (SG, 2x2)

$3.4 e+007$

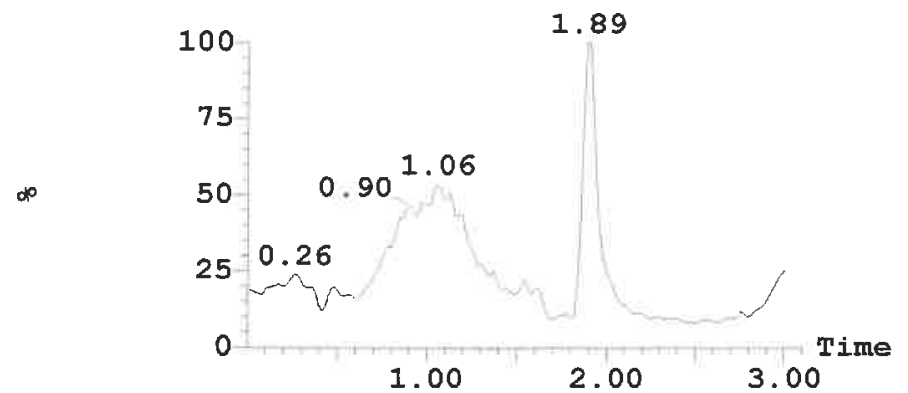

3: UV Detector: TIC

2.389

Range: 2.395

(2) ELSD Signal

1418.285
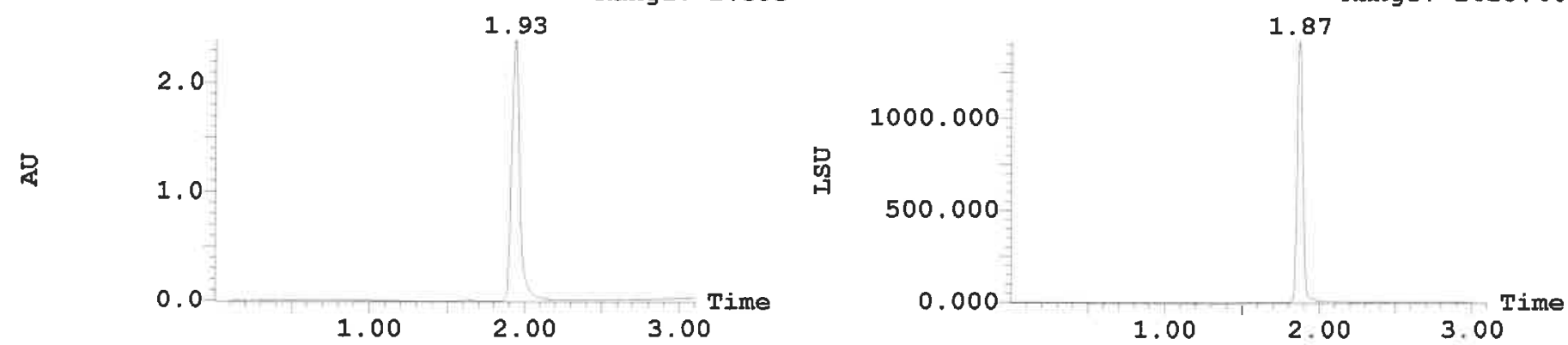
$\begin{array}{rr}\text { Peak ID Compound } & \text { Time } \\ 1 & 0.72\end{array}$ Mass Found

$1:$ MS ES+

$1.2 e+006$

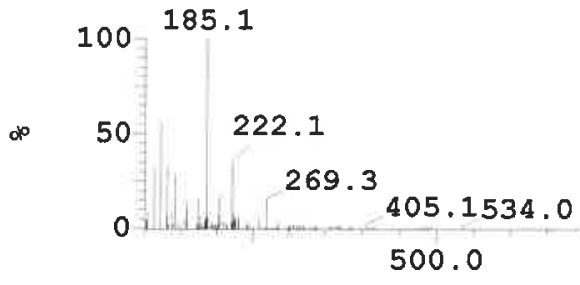

Peak ID Compound Time Mass Found

3 1.90
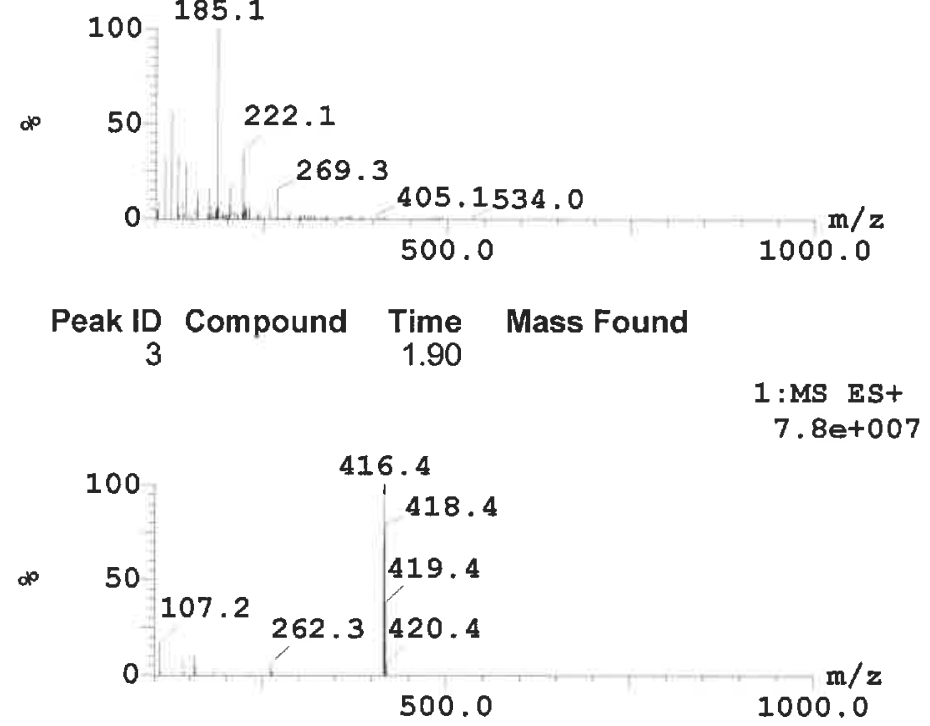

\section{$\begin{array}{rrr}\text { Peak ID } & \text { Compound } & \text { Time } \\ 2 & 0.79 & \text { Mass Found }\end{array}$}

$1:$ MS ES+ $7.1 \mathrm{e}+005$
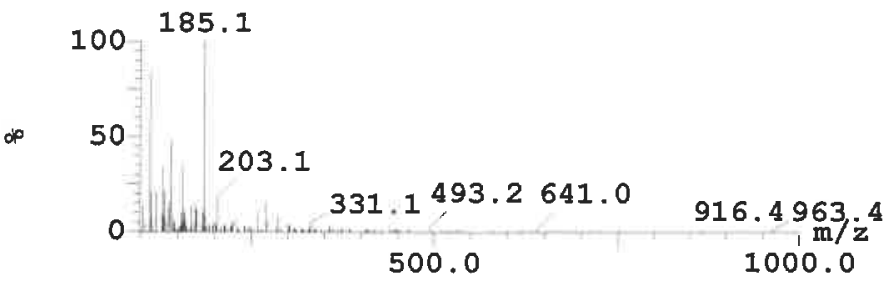

Peak ID Compound Time Mass Found 2.89 1 :MS ES+ 2. 1e+006

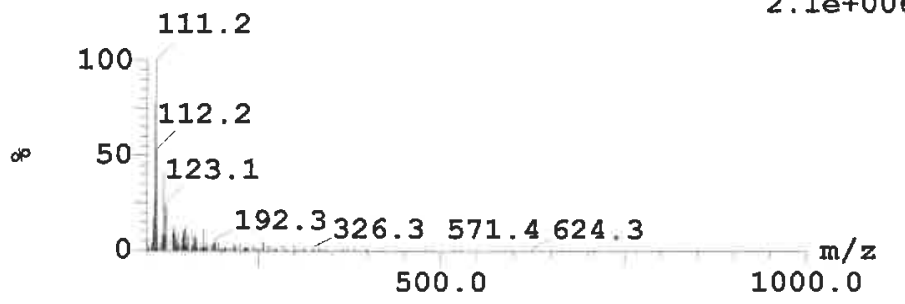


Vial: $1: 31$

Time:11:02:12

\section{Description:}

1: MS ES+ :TIC Smooth (SG, 2×2)

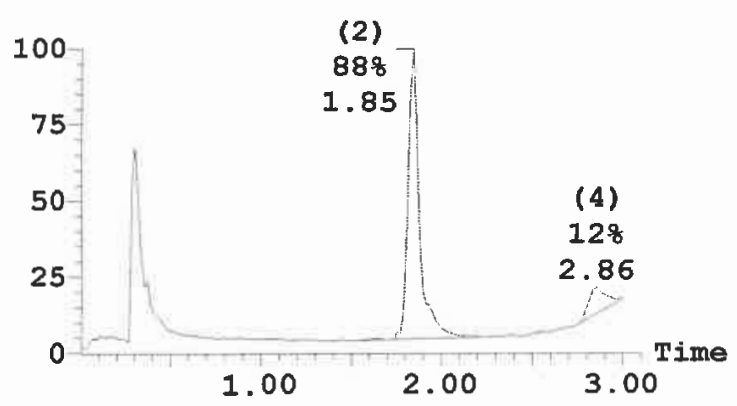

3: UV Detector: TIC Range: $1.513 e+2$ 1.90

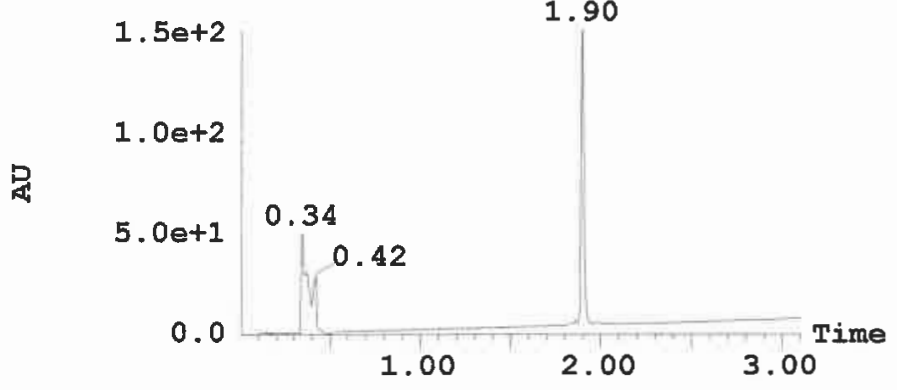

Peak ID Compound Time Mass Found

\begin{tabular}{rr|r}
100 & 143.1 \\
& 50 & 161.6
\end{tabular}

(3) $\quad 279.2336 .3507 .6$

$\begin{array}{cc}0 & 777.3922 .3^{994.3} \\ 500.0 & 1000.0\end{array}$

Peak ID Compound Time Mass Found 3 2.16

120.1

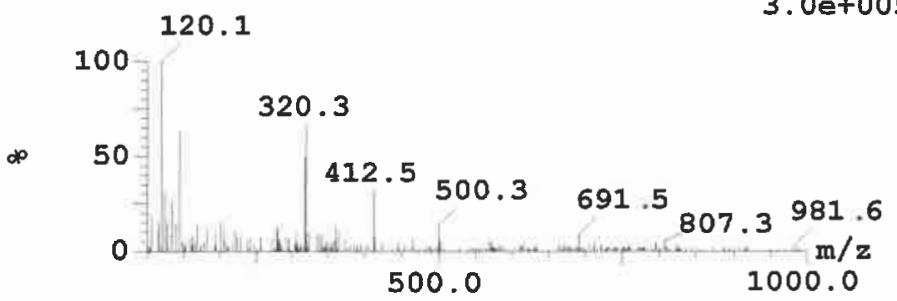

$1.508 e+2$

1 : MS ES+

7. $9 e+005$

(1) ELSD Signal

$4.4 e+007$

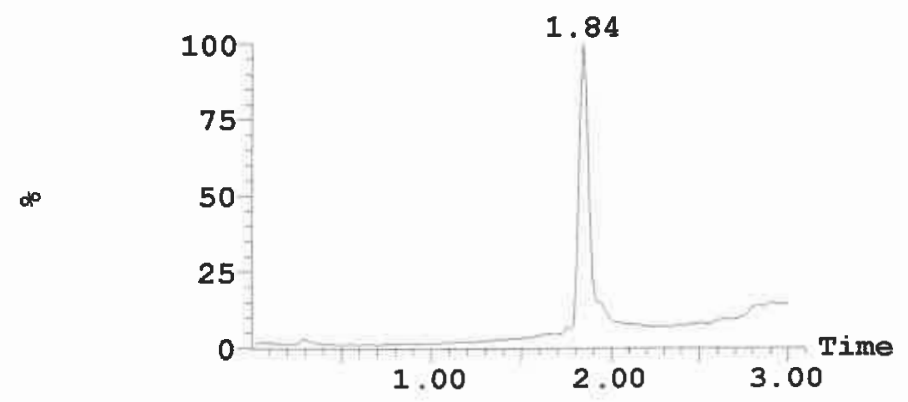

283.833

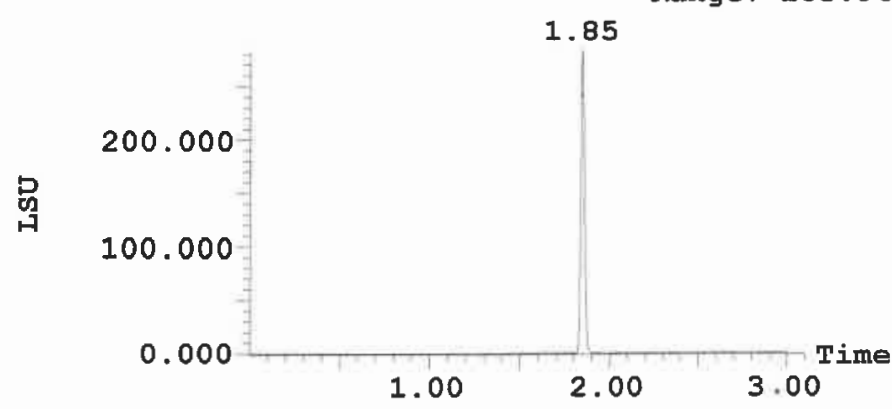

\section{Peak ID Compound Time
2}

1 :MS ES+

$5.7 e+007$

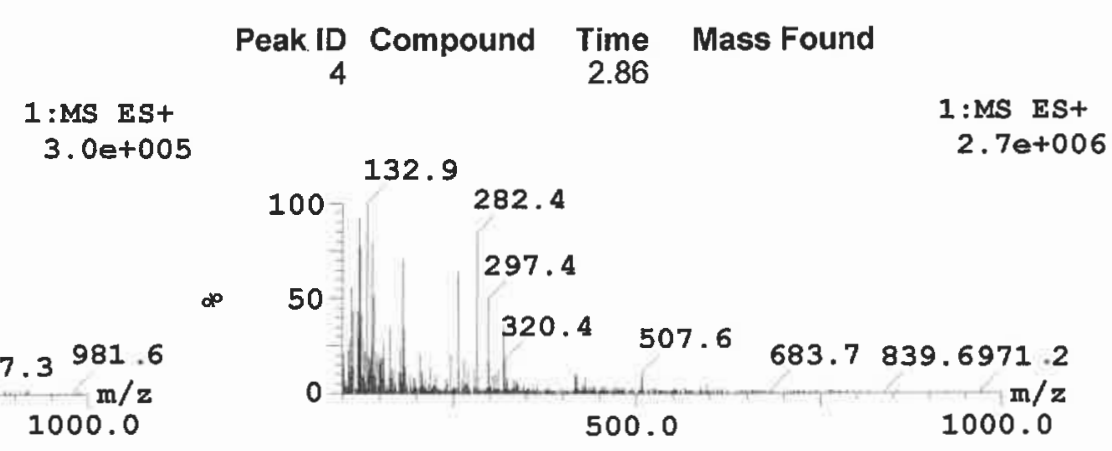


Vial: $2: 9$

Time:12:33:50

\section{Description:}

1: MS ESt :TIC Smooth (SG, 2×2)

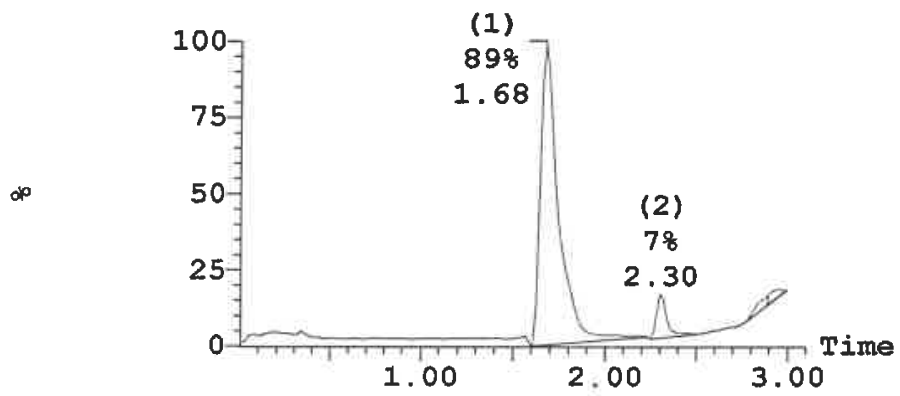

3: UV Detector: TIC

$5.019 e+1$ Range: 5.506e+1

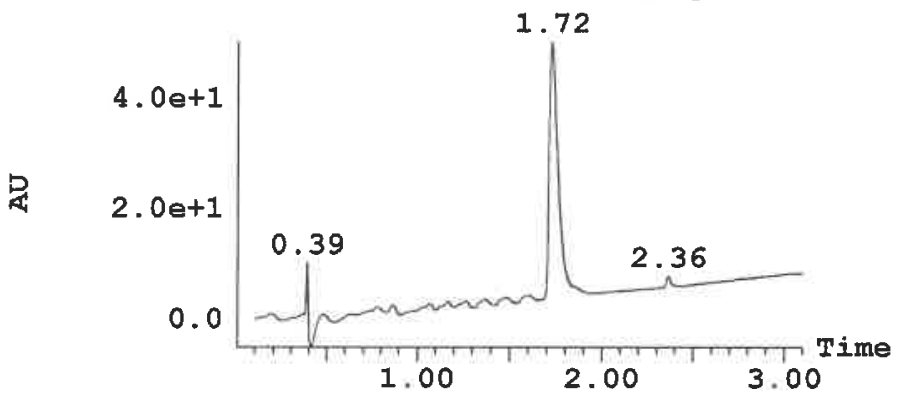

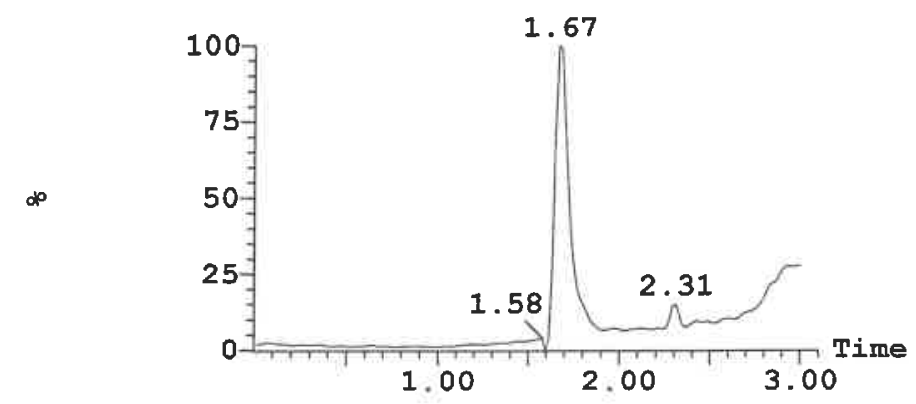

(1) ELSD Signal
19.915

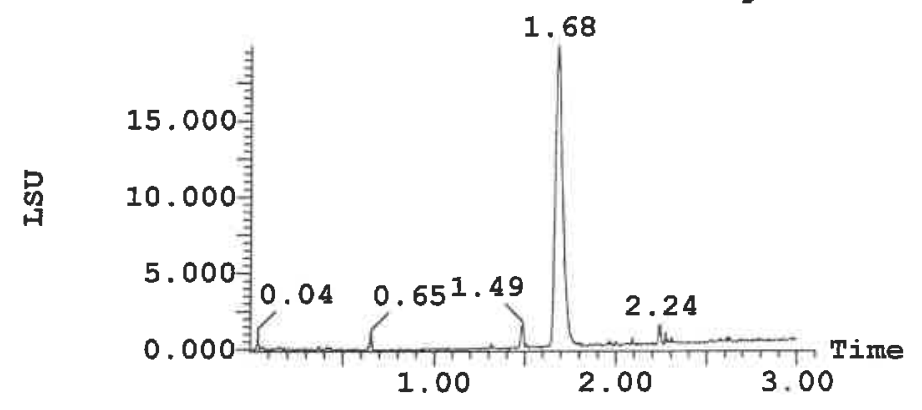

\section{Peak ID Compound Time
1.68 Mass Found}

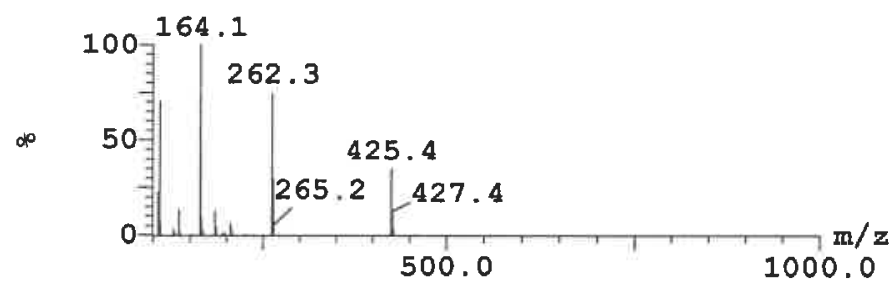

1 : MS ES+
$6.30+007$

Peak ID Compound $\begin{array}{r}\text { Time } \\ 2.30\end{array} \quad$ Mass Found
2.30

$1:$ MS ES+

8. $5 e+006$

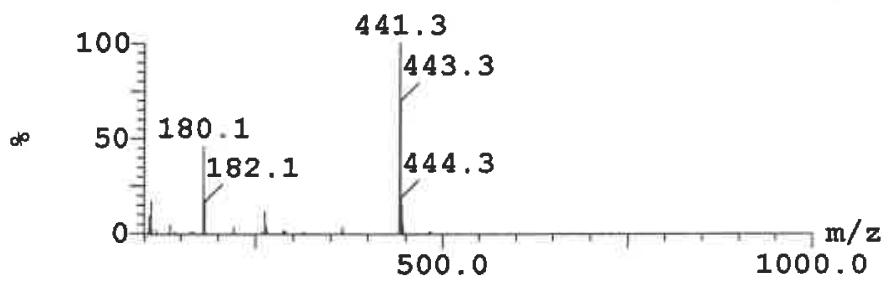

\section{Peak ID Compound
4 $\begin{array}{r}\text { Time } \\ 2.94\end{array} \quad$ Mass Found}

1 : MS ES+

$4.3 e+006$

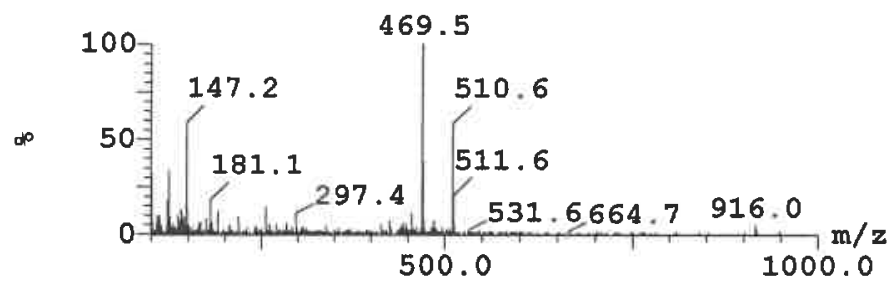

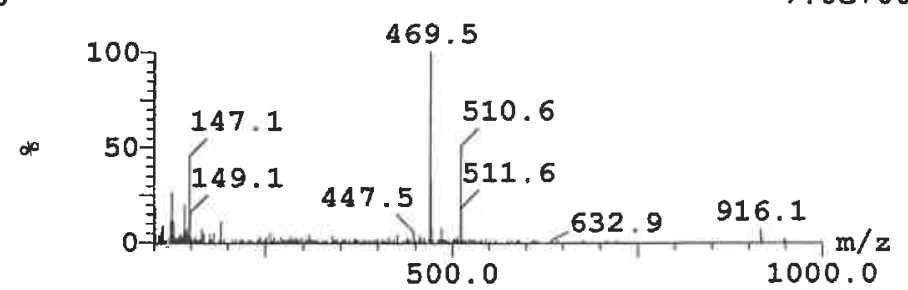




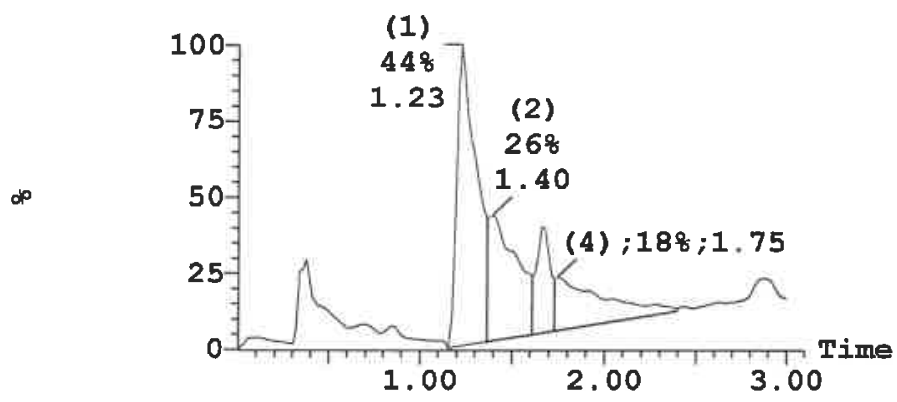

3: UV Detector: TIC

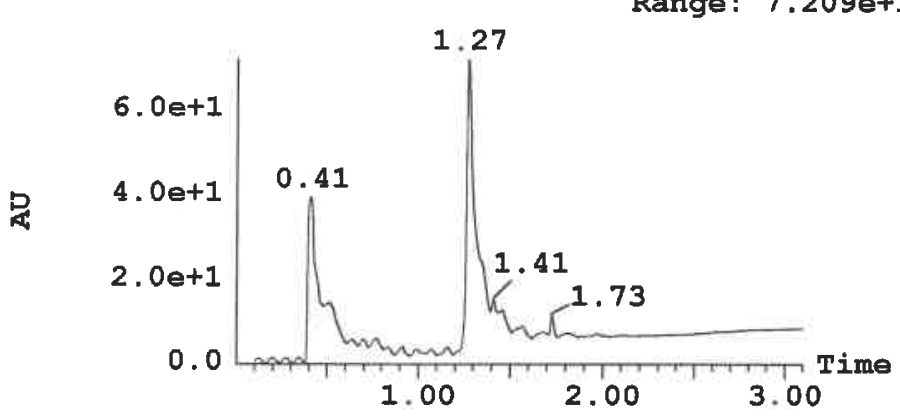

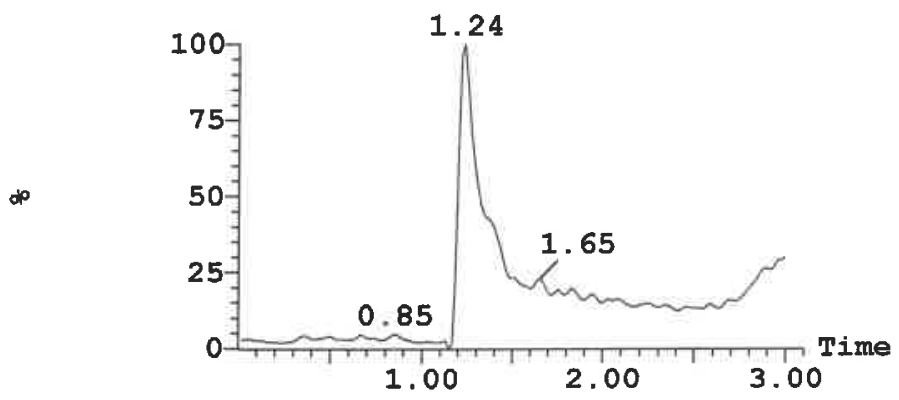

(1) ELSD Signal

40.051

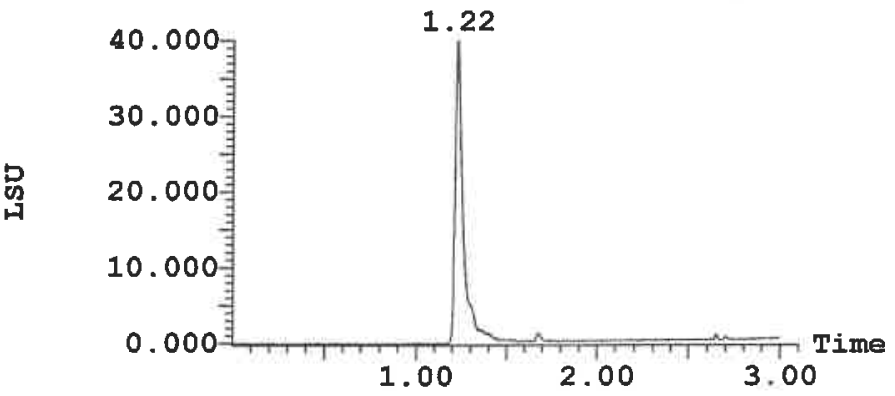

Peak ID Compound Time
1

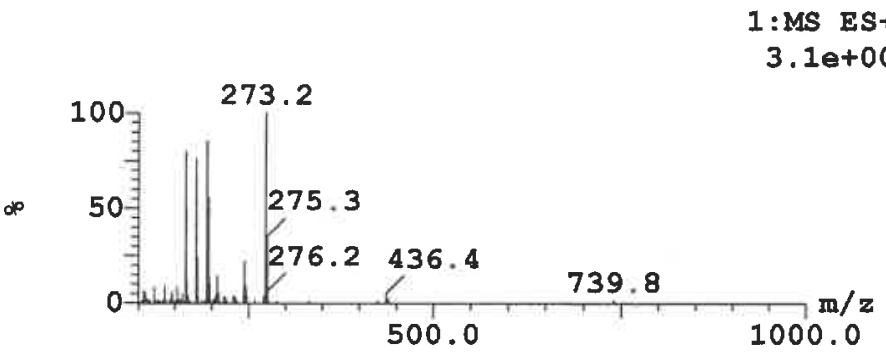
Peak ID Compound Time
1.40 Mass Found

$1:$ MS ES+ 1. 0 et 007

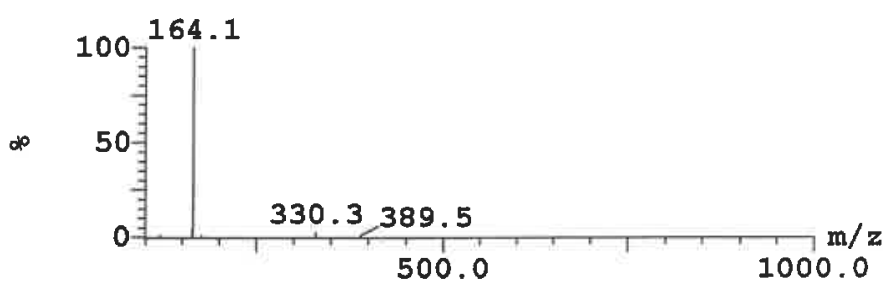

Peak ID Compound
4 $\quad \begin{aligned} 1.75 & \text { Mass Found }\end{aligned}$

1:MS ES+

$1.4 e+007$

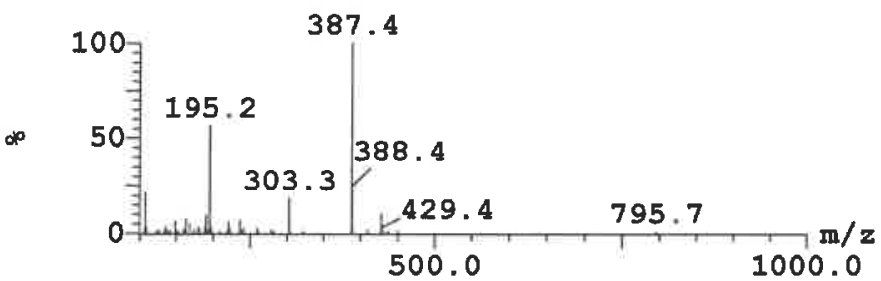

1:MS ES+

4. 0 et +006

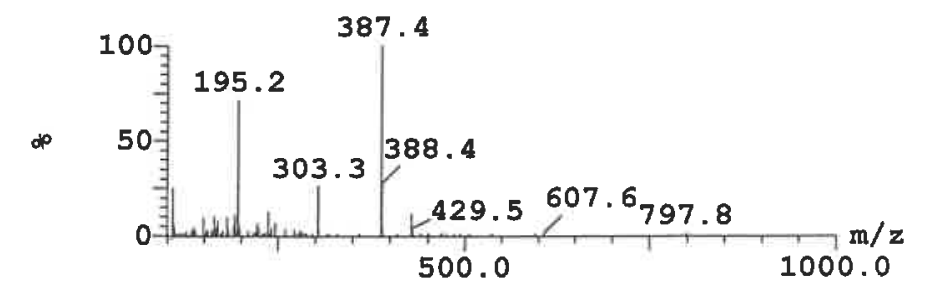




\section{3) References.}

(1) Kiefersauer, R.; Than, M.; Dobbek, H.; Gremer, L.; Melero, M.; Strobl, S.; Dias, J.; Soulimane, T.; Huber, R. A Novel Free-Mounting System For Protein Crystals: Transformation And Improvement Of Diffraction Power By Accurately Controlled Humidity Changes. Journal of Applied Crystallography 2000, 33, 1223-1230.

(2) Kabsch, W. XDS. Acta Crystallographica Section D Biological Crystallography 2010, 66, $125-132$.

(3) McCoy, A.; Grosse-Kunstleve, R.; Adams, P.; Winn, M.; Storoni, L.; Read, R. Phaser Crystallographic Software. Journal of Applied Crystallography 2007, 40, 658-674.

(4) Emsley, P.; Lohkamp, B.; Scott, W.; Cowtan, K. Features And Development Of Coot. Acta Crystallographica Section D Biological Crystallography 2010, 66, 486-501.

(5) Murshudov, G.; Vagin, A.; Dodson, E. Refinement Of Macromolecular Structures By The Maximum-Likelihood Method. Acta Crystallographica Section D Biological Crystallography 1997, 53, 240-255.

(6) Battye, T.; Kontogiannis, L.; Johnson, O.; Powell, H.; Leslie, A. iMOSFLM: A New Graphical Interface For Diffraction-Image Processing With MOSFLM. Acta Crystallographica Section D Biological Crystallography 2011, 67, 271-281.

(7) Liebschner, D.; Afonine, P.; Baker, M.; Bunkóczi, G.; Chen, V.; Croll, T.; Hintze, B.; Hung, L.; Jain, S.; McCoy, A.; Moriarty, N.; Oeffner, R.; Poon, B.; Prisant, M.; Read, R.; Richardson, J.; Richardson, D.; Sammito, M.; Sobolev, O.; Stockwell, D.; Terwilliger, T.; Urzhumtsev, A.; Videau, L.; Williams, C.; Adams, P. Macromolecular Structure Determination Using X-Rays, Neutrons And Electrons: Recent Developments In Phenix. Acta Crystallographica Section D Structural Biology 2019, 75, 861-877. 